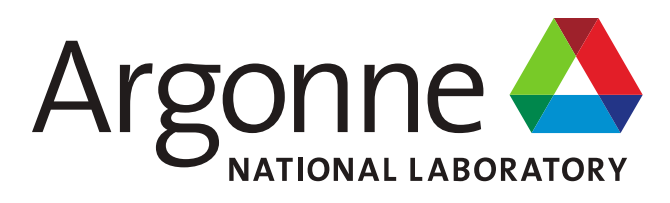

ANL/RTR/TM-20/1

\title{
Impact of MURR LEU Conversion on Beryllium Reflector Lifetime
}

Nuclear Science \& Engineering Division 


\title{
About Argonne National Laboratory
}

Argonne is a U.S. Department of Energy laboratory managed by UChicago Argonne, LLC under contract DE-AC02-06CH11357. The Laboratory's main facility is outside Chicago, at 9700 South Cass Avenue, Argonne, Illinois 60439. For information about Argonne and its pioneering science and technology programs, see www.anl.gov.

\section{DOCUMENT AVAILABILITY}

Online Access: U.S. Department of Energy (DOE) reports produced after 1991 and a growing number of pre-1991 documents are available free at OSTI.GOV (http://www.osti.gov/), a service of the U.S. Dept. of Energy's Office of Scientific and Technical Information.

\author{
Reports not in digital format may be purchased by the public from the \\ National Technical Information Service (NTIS): \\ U.S. Department of Commerce \\ National Technical Information Service \\ 5301 Shawnee Rd \\ Alexandria, VA 22312 \\ www.ntis.gov \\ Phone: (800) 553-NTIS (6847) or (703) \\ 605-6000 Fax: (703) 605-6900 \\ Email: orders@ntis.gov
}

\section{Reports not in digital format are available to DOE and DOE contractors from the Office of Scientific and Technical Information (OSTI):}

U.S. Department of Energy

Office of Scientific and Technical Information

P.O. Box 62

Oak Ridge, TN 37831-0062

www.osti.gov

Phone: (865) 576-8401

Fax: (865) 576-5728

Email: reports@osti.gov

Disclaimer

\section{Disclaimer}

This report was prepared as an account of work sponsored by an agency of the United States Government. Neither the United States Government nor any agency thereof, nor UChicago Argonne, LLC, nor any of their employees or officers, makes any warranty, express or implied, or assumes any legal liability or responsibility for the accuracy, completeness, or usefulness of any information, apparatus, product, or process disclosed, or represents that its use would not infringe privately owned rights. Reference herein to any specific commercial product, process, or service by trade name, trademark, manufacturer, or otherwise, does not necessarily constitute or imply its endorsement, recommendation, or favoring by the United States Government or any agency thereof. The views and opinions of document authors expressed herein do not necessarily state or reflect those of the United States Government or any agency thereof, Argonne National Laboratory, or UChicago Argonne, LLC. 


\title{
Impact of MURR LEU Conversion on Beryllium Reflector Lifetime
}

\author{
prepared by \\ Cezary Bojanowski, John Stillman, Walid Mohamed, Earl Feldman, and Erik Wilson \\ Nuclear Science \& Engineering Division, Argonne National Laboratory \\ Les Foyto \\ University of Missouri Research Reactor \\ Gary Solbrekken \\ University of Missouri
}

September 2020 
(This page left intentionally blank) 


\section{Executive Summary}

The U.S. National Nuclear Security Administration (NNSA) Office of Material Management and Minimization $\left(\mathrm{M}^{3}\right)$ has developed and is pursuing an integrated approach to address the persistent threat posed by unintentional proliferation of nuclear materials. The NNSA $\mathrm{M}^{3}$ approach reduces the risk of highly enriched uranium (HEU) and plutonium falling into the hands of non-state actors by minimizing the use of and, when possible, eliminating weapons-usable nuclear material around the world. In this geopolitical context, most research and test reactors, both domestic and international, have completed or started a program of conversion from the use of HEU to low-enriched uranium (LEU) as fuel.

Conversion of civilian research reactors from HEU to LEU, and the return of the HEU to the country of origin, is an important component of the NNSA non-proliferation program. Worldwide, 71 reactors have been converted to the use of LEU fuel, and an additional 28 have been confirmed to be permanently shut down. The U.S., with 20 reactor conversions, is among the 39 countries on six continents where conversions have occurred. With recent conversions in Ghana and Nigeria, an important milestone was reached in completing conversion of all reactors on the continent of Africa to LEU fuel. Africa thus becomes the third continent to have completed conversion of all HEU reactors to LEU, following Australia and South America.

As part of NNSA's HEU minimization mission, the NNSA M ${ }^{3}$ Conversion Program is collaborating with six U.S. High Performance Research Reactors (USHPRRs), including one critical facility, to convert from the use of HEU to LEU fuel. The $\mathrm{M}^{3}$ conversion objectives for the USHPRRs are to develop LEU fuel element designs that will ensure safe reactor operations, as well as maintain the existing experimental performance of each facility. The work is being conducted through many interrelated activities that are being completed by stakeholders across organizations.

A new type of LEU fuel based on an alloy of uranium-10wt\% molybdenum (U-10Mo) is expected to allow the conversion of those USHPRRs requiring higher-density fuels. This very-high-density monolithic fuel is currently undergoing irradiation and post-irradiation testing under a planned and documented fuel qualification program. The Fuel Qualification (FQ) Pillar of the USHPRR Project will document fuel properties and fuel performance data and qualify the fuel for use. The Fuel Fabrication (FF) Pillar is fabricating fuel for ongoing and future irradiation tests, as well as conducting fabrication demonstrations to validate or update preliminary fabrication assumptions. The FF Pillar is also working to develop and install manufacturing capacity for the U-10Mo monolithic fuel.

Within the Reactor Conversion Pillar of the USHPRR Project, four of the USHPRRs, including the University of Missouri Research Reactor (MURR®), have progressed through preliminary element design using the proposed monolithic alloy of U-10Mo. Preliminary fuel element design and safety analyses have been completed for MURR. This work has relied on preliminary data for properties, performance, and fabrication tolerances for the fuel systems that have been produced by the FQ and FF Pillars.

The MURR core is surrounded by a beryllium reflector. The first MURR beryllium reflector was designed with two beamport holes located near the mid-height of the reflector. It failed in May of 1981 at around 27,100 MWd of operation. The reflector fractured into two parts, upper and lower, separated by a crack at the height of the beamport holes. To preclude the likelihood of failure, all subsequent reflectors have been replaced after a predetermined period of use. An operating limit for 
the beryllium reflector of 26,000 MWd was selected, which corresponds to approximately 8 years of operation for the reactor in a core operating at $10 \mathrm{MW}$.

Former studies indicate that for the proposed MURR LEU-fueled core, the steady-state operating power must be increased from $10 \mathrm{MW}$ to $12 \mathrm{MW}$ in order to maintain the same level of neutron flux after conversion at various experimental locations in the reactor. The increase in the operating power of the reactor, together with the change of fuel system, will considerably affect the neutron fluence, damage to the internal structure of the beryllium material, and the He-4 production in the beryllium reflector that is causing the swelling of beryllium. Before the conversion, it must be determined whether the beryllium reflector with the LEU core and power uprate to $12 \mathrm{MW}$ will be able to be used for the same operating period as the current HEU core at $10 \mathrm{MW}$.

The work described in this report consists of three parts: (1) neutronics calculations of HEU and LEU cores at 8 years of operation, (2) an extensive literature review to gather the data regarding degradation of irradiated-beryllium material properties, and (3) finite element (FE) analysis of the MURR reflector aiming at predicting the state of stress in the reflector due to irradiation-induced heating, swelling, and material property degradation.

Neutronics calculations have been performed that predict the rate of heating, He-4 gas production, and material damage in dpa for both the HEU and LEU cores. The changes are largely driven by the $20 \%$ power uprate planned for the LEU conversion, and the reduction in the heating ratedue to the additional heavy metal in the LEU core as compared to the HEU core. These calculations are reported and used as input data allowing FE modeling to be performed, and as input into properties correlations as described below.

According to a review of the literature, the transmutation-gas-induced swelling rate, as well as degradation of some thermo-mechanical beryllium properties (including thermal conductivity) due to irradiation, appear to be independent of beryllium grade. Strength properties, like yield and ultimate tensile and compressive strengths, are highly dependent on beryllium grade. Thus, properties of irradiated beryllium obtained for grades other than the one currently used at MURR (S200-FH) may exhibit similar trends in the degradation, but quantification of the life span of the MURR reflector may have high uncertainty associated with it where such properties from other grades than S-200-FH are used in the analysis. This idea may be examined through testing if the conclusions regarding the beryllium reflector lifetime require higher-fidelity data than is presently available.

Beryllium subject to irradiation undergoes an embrittlement process, initially losing its ductility and gaining ultimate tensile strength (UTS), but eventually the UTS decreases well below the strength of fresh (i.e., unirradiated) beryllium. Constantly increasing swelling-induced stresses and decreasing strength are the two major factors influencing the lifetime of the beryllium reflector.

The FE analysis presented in this report indicates that the tensile stresses on the outer surface of the reflector at its mid-height are decisive in determining the lifetime of the reflector at MURR. These stresses most likely caused the failure of the first MURR beryllium reflector in 1981, in which cracks propagated from the edges of the beamport holes around the reflector, splitting it into two parts. The second reflector, installed in 1981, had exactly the same design and was made of the same beryllium grade. It did not fail in its 8 years and 26,000 MWd of operations. This observation indicates that quality of surface and presence of cracks in the beryllium block contributed to the failure of the first reflector. 
Starting with the third MURR reflector, the beamport holes were removed from the design of the reflector and the grade of beryllium used to manufacture the reflector was changed (from N-200-A to S-200-FH). S-200-FH grade has more isotropic properties than N-200-A and has higher strength in the fresh, unirradiated state. This change in the design contributed to a significant decrease in the tensile stresses on the outer surface of the reflector. Analysis presented here estimated up to 38\% reduction of tensile stresses at key locations. The FE analysis also indicates that tensile stresses in the reflector (without beamport holes) operating in an LEU core may increase by up to $5 \%$ as compared to the case with an HEU core and reflector without the beamport hole. However, these maximum tensile stresses in the LEU case without beamport holes will still be about $36 \%$ lower than the stresses predicted for the case of the reflector with beamport holes in its design. In this case, modeling performed for the LEU conversion is able to analyze effects that, although slightly less favorable than the HEU case, were not quantified previously and so can lead to realizable margins for assessed lifetimes of beryllium reflectors with both LEU and HEU cores.

The largest compressive stresses are present in the reflector on the bottom of the spacer grooves at the mid-height. These stresses for the LEU case could be about $10 \%$ higher than the highest compressive stresses at the same location for MURR beryllium reflectors operating with an HEU core (both the old design with beamport holes and the designs without them). These stresses, although very high as compared to the ultimate compressive strength, are not of primary concern, as the compressive stresses do not contribute significantly to crack propagation.

Although the analysis predicts that there was a gain in the safety margin when the beamport holes were removed, it cannot be determined precisely whether and by how many years the replacement schedule for the reflector can be extended beyond the current practice of 8 years of operation. UTS degradation data and fracture toughness data for relevant levels of irradiation with the S-200-FH grade could be measured if more precise predictions are needed to extend the reflector lifetime for HEU or LEU operations. Also, additional understanding of initial cracks present on the surface of materials must be acquired, and extensive studies of these cracks may potentially be needed in order to establish a link of newly acquired data to failures that would potentially need to be induced, either for the S-200-FH grade or more generally. Regardless, the analysis showed that beryllium reflector lifetimes with both the HEU and LEU cores would exceed the current operational limit of 26,000 MWd achieved at 8 years of operation. 


\section{Table of Contents}

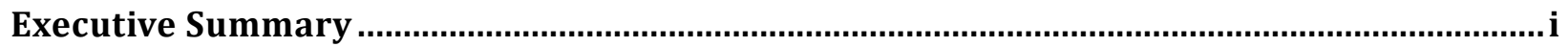

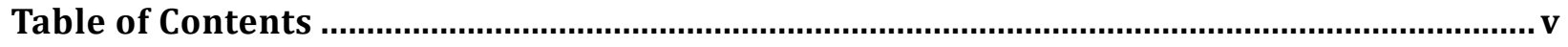

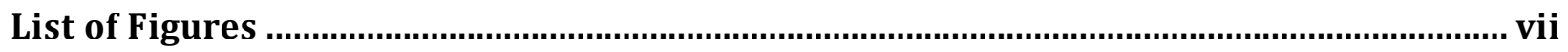

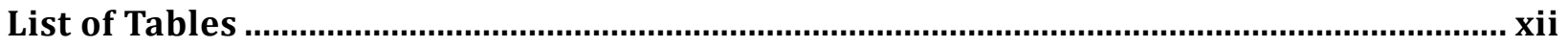

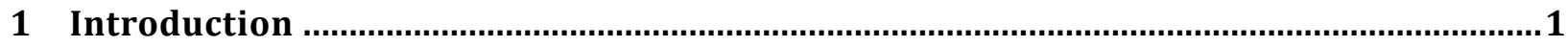

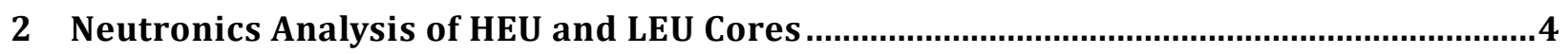

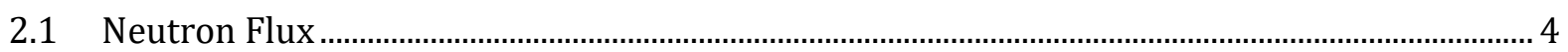

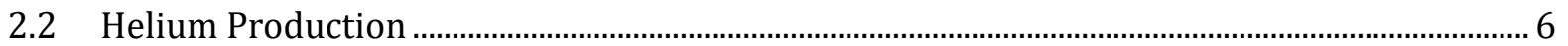

2.3 Irradiation Damage (Displacements per Atom) ……...................................................................... 8

2.4 Neutron and Photon Heat Generation...................................................................................................10

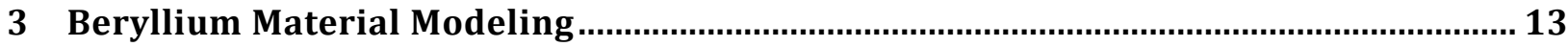

3.1 Material Properties of Fresh (Unirradiated) Beryllium .................................................................13

3.2 Effect of Temperature on Material Properties of Fresh (Unirradiated) Beryllium .................17

3.3 Effect of Irradiation on Material Properties of Beryllium ...........................................................22

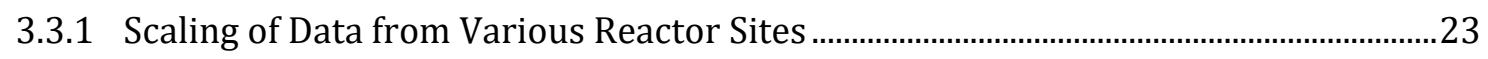

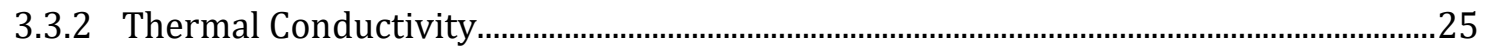

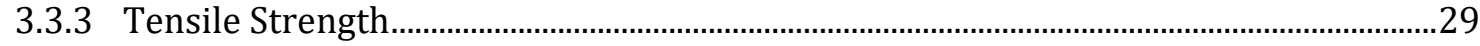

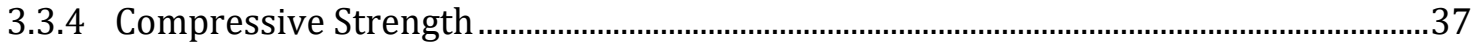

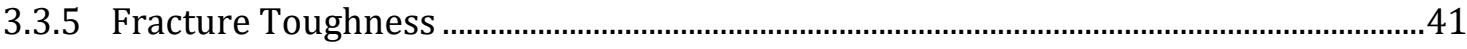

3.4 Volumetric Swelling Model of Irradiated Beryllium ...................................................................4 44

3.5 Selection of Failure Criterion for Irradiated Beryllium ................................................................46

4 Finite Element Model of MURR Beryllium Reflector ................................................. 53

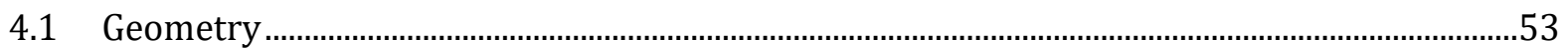

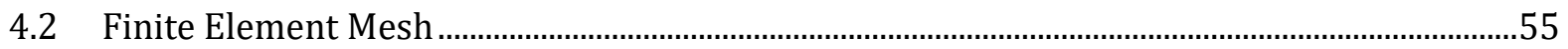

4.2.1 Size of Cells across the Thickness of the Reflector ..................................................................55

4.2.2 Size of Cells along the Height of the Model............................................................................56

4.2.3 Number of Cells along the Groove Curvature ..........................................................................57

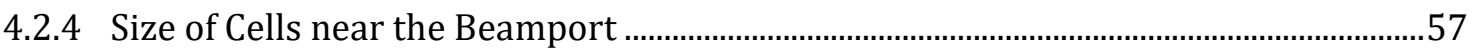

4.3 Mechanical Boundary Conditions and Loadings..........................................................................59

4.4 Thermal Boundary Conditions and Loadings ..........................................................................63

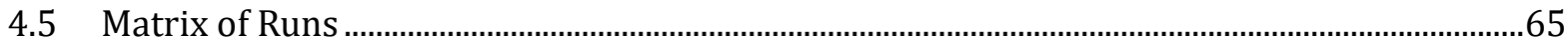

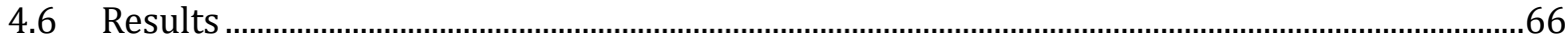

4.6.1 Stresses in Beryllium (N-200-A) Reflectors with HEU Core and Two Beamport Holes 66 
4.6.2 Stresses in Beryllium (S-200-FH) Reflectors with HEU Core and No Beamport Holes 70

4.6.3 Stresses in Future Beryllium (S-200-FH) Reflectors with LEU Core and No Beamport Holes 73

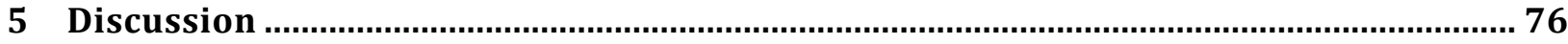

5.1 Tensile Stresses in Beryllium Reflectors ……...................................................................................

5.2 Compressive Stresses in Beryllium Reflectors............................................................................84

5.3 Stress Intensity in Beryllium Reflectors and Critical Crack Length ............................................85

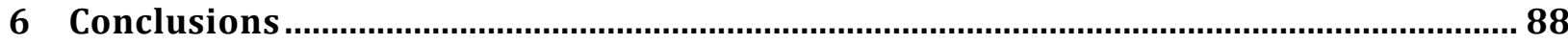

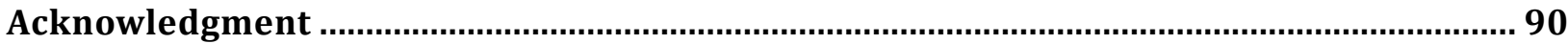

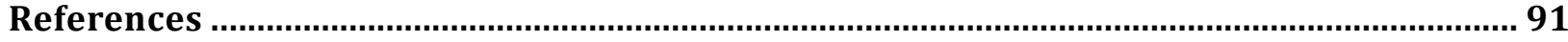

APPENDIX A: MURR Beryllium Reflector Flux, Gas Production, Damage, and Heating.... 97

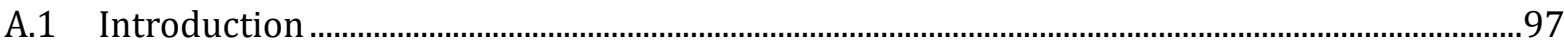

A.2 Relevant Reactions for Gas Production in Irradiated Beryllium ................................................98

A.3 Neutron Flux: Beryllium Reflector with HEU Core ....................................................................99

A.4 Helium Production: Beryllium Reflector with HEU Core.............................................................. 104

A.5 Neutron Irradiation Damage: Beryllium Reflector with HEU Core ........................................... 110

A.6 Neutron and Photon Heating: Beryllium Reflector with HEU Core........................................... 112

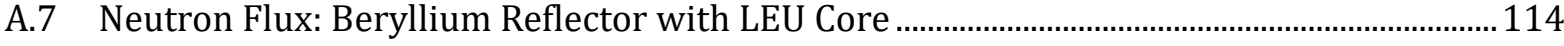

A.8 Helium Production: Beryllium Reflector with LEU Core ……….............................................115

A.9 Irradiation Damage in the Beryllium Reflector with LEU Core................................................... 117

A.10 Neutron and Photon Heating: Beryllium Reflector with LEU Core ...........................................118

A.11 Azimuthal Distribution of Irradiation Conditions in Beryllium Reflector..............................119

A.12 Summary …….....................................................................................................................

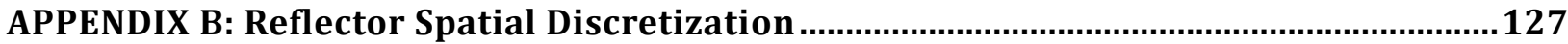

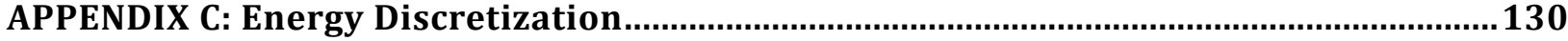

APPENDIX D: Helium Concentration, Neutron Damage, and Heating in Beryllium Reflector 131

APPENDIX E: Conversion of Data from Various Reactor Sites ............................................143

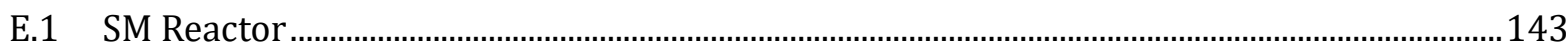

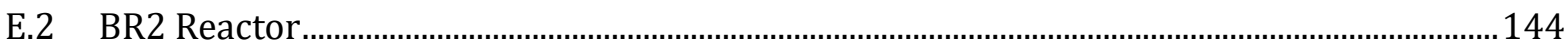

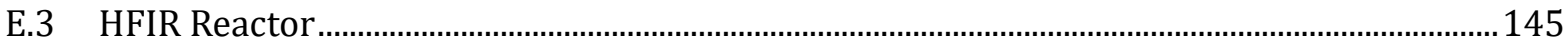




\section{List of Figures}

Figure 1.1. Sketch of MURR components in vicinity of beryllium reflector. 2

Figure 1.2. MURR beryllium reflector: basic dimensions (left) and schematic of crack location in reflector from 1981 (right)....

Figure 2.1. Distribution of total flux (left) and fast fluence ( $\mathrm{E}>1 \mathrm{MeV})$ after 8 years (right) in beryllium reflector with HEU core..

Figure 2.2. Distribution of total flux (left) and fast fluence $(\mathrm{E}>1 \mathrm{MeV})$ after 8 years (right) in beryllium reflector with LEU core 5

Figure 2.3. Fast fluence $(\mathrm{E}>1.0 \mathrm{MeV})$ through the reflector at mid-height after 8 years with $\mathrm{HEU}$ and LEU cores 6

Figure 2.4. Distribution of He-4 content in beryllium reflector at 8 years with HEU core (in units of appm). Data imported to COMSOL (left); data extrapolated on the beryllium reflector model (right).

Figure 2.5. Distribution of He-4 content in beryllium reflector at 8 years with LEU core (in units of appm). Data imported to COMSOL (left); data extrapolated on the beryllium reflector model (right).

Figure 2.6. He-4 concentration through the reflector at mid-height with HEU and LEU cores after 8 years. 8

Figure 2.7. Distribution of damage in beryllium reflector with HEU core at 8 years of operation (in units of dpa). Data imported to COMSOL (left); data extrapolated on the beryllium reflector model (right).

Figure 2.8. Distribution of damage in beryllium reflector with LEU core at 8 years of operation (in units of dpa). Data imported to COMSOL (left); data extrapolated on the beryllium reflector model (right). ...10

Figure 2.9. Damage through the reflector at mid-height for HEU and LEU cores 10

Figure 2.10. Distribution of internal photon heating in the beryllium reflector with the HEU core $\left(\mathrm{W} / \mathrm{cm}^{3}\right)$. Data imported to COMSOL (left); data extrapolated on the beryllium reflector model (right). . .11

Figure 2.11. Distribution of photon heating in beryllium reflector with the LEU core $\left(\mathrm{W} / \mathrm{cm}^{3}\right)$. Data imported to COMSOL (left); data extrapolated on the beryllium reflector model (right)......................12

Figure 2.12. Internal heating through the reflector at mid-height for HEU and LEU cores ...................12 Figure 3.1. Stress-strain curves from tensile-strength test for S-200-F grade (left) and compressivestrength test at room temperature for S-200-FH grade (right)... 17

Figure 3.2. Temperature dependency of Young's modulus and elongation at failure for S-200-F grade

Figure 3.3. Dependency of yield strength and UTS on temperature for S-200-FH (left) and S-200-F (right) beryllium . . .18

Figure 3.4. Coefficient of thermal expansion of S-200-F grade (left) and two other unspecified grades produced by Materion with different $\mathrm{BeO}$ contents (right). 19

Figure 3.5. Thermal conductivity of hot-pressed beryllium produced by Brush Wellman (Materion's predecessor) in the 1970s (left) and produced more recently by Materion (right) 20 
Figure 3.6.Thermal conductivity of several beryllium grades produced in Russia 20

Figure 3.7. Dependency of fracture toughness of beryllium on temperature: collective data for S-200F, S-200-FH, S-65, S-65-H grades (left); data for S-200-E (right). .21

Figure 3.8. Dependency of fracture toughness of beryllium on temperature for CIP-HIP grade..........21 Figure 3.9. Relationship between fluence $(\mathrm{E}>1.0 \mathrm{MeV}$ ) and He-4 content (left) and fluence (E $>1.0$ $\mathrm{MeV}$ ) and damage (right) in MCNP5 calculations for MURR beryllium reflector .25 Figure 3.10. Thermal conductivity of irradiated beryllium as a function of fast neutron fluence $(\mathrm{E}>$ $0.1 \mathrm{MeV}$ )

Figure 3.11. Dependence of the thermal conductivity of TE-56 grade beryllium on testing temperature

Figure 3.12. Thermal conductivity of irradiated beryllium as a function of damage in dpa, with a fit for S-200-FH grade beryllium.

Figure 3.13. Thermal conductivity of irradiated beryllium as a function of damage in dpa, with a fit for N-200-A grade beryllium.

Figure 3.14. Thermal conductivity distribution in beryllium reflector irradiated for 8 years at $10 \mathrm{MW}$ with HEU core.

Figure 3.15. Thermal conductivity distribution in beryllium reflector irradiated for 8 years at $12 \mathrm{MW}$ with LEU core.

Figure 3.16. Raw data from tensile tests of S-200-FH grade, digitized from (left) and stress-strain curves from tensile tests of S-65-C grade, digitized from (right). 30

Figure 3.17. Dependency of yield strength and UTS of S-200-FH grade beryllium on temperature for unirradiated and irradiated samples, with irradiation up to a fluence $(E>1.0 \mathrm{MeV})$ of $0.15^{*} 10^{22} \mathrm{n} / \mathrm{cm}^{2}$ [47].

Figure 3.18. Dependency of yield strength and UTS of S-200-F grade beryllium on temperature for unirradiated and irradiated samples, with irradiation up to a fluence $(\mathrm{E}>1.0 \mathrm{MeV})$ of $0.15^{*} 10^{22}$ $\mathrm{n} / \mathrm{cm}^{2}$.

Figure 3.19. Tensile yield strength and UTS data following irradiation at ATR.........................................32

Figure 3.20. UTS data for various beryllium grades tested at the SM reactor ............................................33

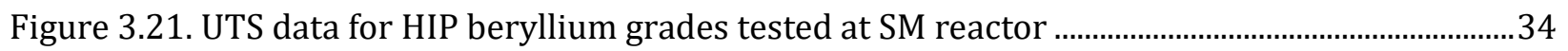

Figure 3.22. UTS data for HIP beryllium grades tested at BR2 and SM reactors as a function of damage

Figure 3.23. UTS degradation data with an assumed fit for S-200-FH and N-200-A grade beryllium35

Figure 3.24. Tensile yield strength and UTS data following irradiation at ATR with assumed UTS degradation model for S-200-FH

Figure 3.25. UTS (MPa) in S-200-FH beryllium reflector irradiated for 8 years with HEU core. Damagedependent fit function implemented in COMSOL (left); data extrapolated on the beryllium model (right).

Figure 3.26. UTS (MPa) in S-200-FH beryllium reflector irradiated for 8 years with LEU core. Damagedependent fit function implemented in COMSOL (left); data extrapolated on the beryllium model (right).

Figure 3.27. Yield strength and UCS for fresh and irradiated beryllium material, based on data from MTR and SM 
Figure 3.28. Yield or UCS for irradiated beryllium material, based on data from ATR, MTR, and SM 38 Figure 3.29. UCS for irradiated beryllium material, based on data from ATR, MTR, and SM as a function of fluence.

Figure 3.30. UCS for irradiated beryllium material, based on data from SM as a function of damage .. .40

Figure 3.31. UCS for irradiated beryllium material based on data from SM, with the polynomial fit to the data. . .40

Figure 3.32. UCS (MPa) in beryllium irradiated for 8 years with MURR HEU core. Damage-dependent fit function implemented in COMSOL (left); data extrapolated on the beryllium model (right).......... 41

Figure 3.33. UCS (MPa) in beryllium irradiated for 8 years with MURR LEU core. Damage-dependent fit function implemented in COMSOL (left); data extrapolated on the beryllium model (right).......... 41

Figure 3.34. Fracture toughness of irradiated beryllium as a function of fluence (E > $1.0 \mathrm{MeV})$......... 42

Figure 3.35. Fracture toughness of irradiated beryllium as a function of $\mathrm{He}-4$ accumulation ...............43

Figure 3.36. Fracture toughness of irradiated beryllium as a function of damage...................................43

Figure 3.37. Volumetric swelling data for irradiated beryllium as a function of He-4 content.............45

Figure 3.38. Swelling in MURR beryllium reflector after 8 years of operation with HEU (left) and LEU

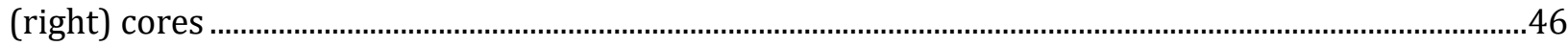

Figure 3.39. Comparison of failure criteria in 2D principal stress space ..................................................48

Figure 3.40. The effect of biaxial stress on yield and UTS of historic KBI beryllium as compared to various failure criteria. 50

Figure 3.41. Stress intensity modification factor for pressurized thick cylinders and cracks on the

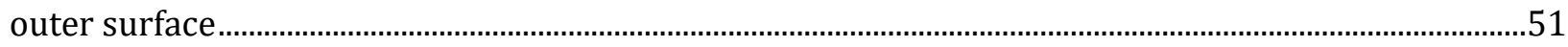

Figure 4.1. Cross section of MURR beryllium reflector with beamport holes ..........................................53

Figure 4.2. Geometry of the beryllium reflector model without beamport holes (left) and with a spacer groove (right) .54

Figure 4.3. Geometry of MURR beryllium reflector with beamport hole (left), and detail of the beamport hole (right) .54

Figure 4.4. Mesh on the model without the beamport hole. .55

Figure 4.5. Various mesh densities in the cross section of the reflector model; from left to right: coarse

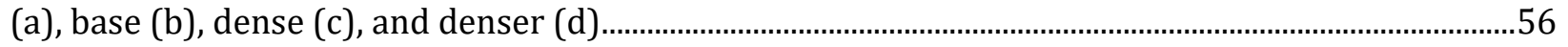

Figure 4.6. Mesh near the spacer groove. From left: coarse (a), base (b), dense (c)...............................57

Figure 4.7. Mesh in the model with beamport holes.........................................................................................58

Figure 4.8. Various mesh densities around the beamport holes: (top) fine, finer, (bottom) extra fine, extremely fine

Figure 4.9. Beryllium reflector and aluminum skirt; (left) photo of MURR reflector with skirt, (right) detail sketch . .60

Figure 4.10. Geometry of beryllium reflector with exact geometry of the base 61

Figure 4.11. Mechanical boundary conditions on the bottom of the reflector. 61

Figure 4.12. Comparison of $1^{\text {st }}$ principal stresses in models with soft (left) and fixed (right) boundary conditions at the bottom of the reflector. 62 
Figure 4.13. $1^{\text {st }}$ principal stresses in the model with exact geometry near the base of the reflector at the mid-height (left) and localized stresses near the base (right). .62

Figure 4.14. Temperature distribution in the FE model (left) and the distribution of temperature through the thickness of the reflector in the hottest location along the y-axis (right), in beryllium reflector with beamport hole after 8 years of operation with HEU core . .66

Figure 4.15. $1^{\text {st }}$ (left) and $2^{\text {nd }}$ (right) principal stresses in the reflector at the location of the beamport hole after 8 years of operation with HEU core .

Figure 4.16. Ratio of $1^{\text {st }}$ principal stress to local UTS in beryllium reflector with beamport hole after 8 years of operation with HEU core 68

Figure 4.17. $1^{\text {st }}$ (left) and $2^{\text {nd }}$ (right) principal stress distribution in beryllium reflector with beamport hole after 8 years of operation with HEU core...

Figure 4.18. $1^{\text {st }}(\mathrm{left})$ and $2^{\text {nd }}$ (right) principal stress distribution with principal directions in beryllium reflector with beamport hole after 8 years of operation with HEU core 69

Figure 4.19. $3^{\text {rd }}$ principal stress distribution in beryllium reflector with beamport hole after 8 years of operation with HEU core 70

Figure 4.20. $3^{\text {rd }}$ principal stress distribution and principal direction in beryllium reflector with beamport hole after 8 years of operation with HEU core 70

Figure 4.21. Temperature distribution in the FE model (left) and the distribution of temperature through the thickness of the reflector in the hottest location along the y-axis (right) in beryllium reflector without beamport hole after 8 years of operation with HEU core... 71

Figure 4.22. $1^{\text {st }}$ principal stress, MPa (left) and ratio of $1^{\text {st }}$ principal stress to local UTS (right) in beryllium reflector without beamport hole after 8 years of operation with HEU core. 71

Figure 4.23. $2^{\text {nd }}$ (left) and $3^{\text {rd }}$ (right) principal stress, MPa, in beryllium reflector without beamport hole after 8 years of operation with HEU core.

Figure 4.24. Principal directions of stresses on the outer surface of the beryllium reflector without beamport hole after 8 years of operation with HEU core 72

Figure 4.25. $3^{\text {rd }}$ principal stresses on the bottom of the groove, with their principal directions, in beryllium reflector without beamport hole after 8 years of operation with HEU core 73

Figure 4.26. Temperature distribution in the FE model (left) and the distribution of temperature through the thickness of the reflector in the hottest location along y-axis (right) in beryllium reflector without beamport hole after 8 years of operation with LEU core . 73

Figure 4.27. $1^{\text {st }}$ principal stress (left) and ratio of $1^{\text {st }}$ principal stress to local UTS (right) in beryllium reflector without beamport hole after 8 years of operation with LEU core. 74

Figure 4.28. $2^{\text {nd }}\left(\mathrm{left}\right.$ ) and $3^{\text {rd }}$ (right) principal stress in beryllium reflector without beamport hole after 8 years of operation with LEU core. 75

Figure 5.1. Locations of highest stresses in the reflector with beamport holes (left) and schematic of crack location in reflector from 1981 (right). 77

Figure 5.2. Relative life prediction for beryllium reflectors with HEU and LEU cores. Note that the plot is not drawn to scale and has only a qualitative character. .79

Figure 5.3. Location of points with highest tensile stresses in beryllium reflector with beamport hole in 2D principal stress after 8 years of operation with HEU core . .80 
Figure 5.4. Location of points with highest tensile stresses in beryllium reflectors without beamport hole in 2D principal stress after 8 years of operation with HEU cores . .80

Figure 5.5. Location of points with highest tensile stresses in beryllium reflectors without beamport hole in 2D principal stress after 8 years of operation with LEU cores 81

Figure 5.6. Progression of maximum stresses with assumed operation time of beryllium reflector $(\mathrm{N}$ 200-A) with beamport holes and HEU core.

Figure 5.7. Progression of maximum stresses with assumed operation time of beryllium reflector (S200-FH) without beamport holes and with HEU core

Figure 5.8. Progression of maximum stresses with assumed operation time of beryllium reflector (S200-FH) without beamport holes and with LEU core

Figure 5.9. $3^{\text {rd }}$ principal stress distribution through the thickness of the beryllium reflector with the LEU core, in the vicinity of the spacer groove

Figure 5.10. Critical crack length in beryllium reflectors at 8, 10, and 12 years of operation with HEU and LEU cores. 


\section{List of Tables}

Table 1.1. Replacement schedule for MURR beryllium reflectors …….......................................................... 2

Table 3.1. Properties of fresh beryllium produced by Materion ......................................................................15

Table 3.2. Material properties assumed for the FE model at fresh, unirradiated state. .........................21

Table 3.3. Relationships between fluence, He-4 content, and dpa for irradiated beryllium at various

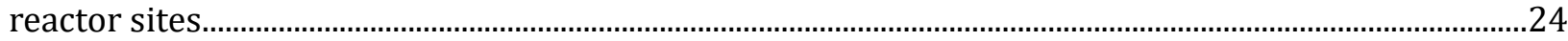

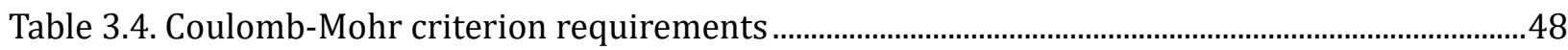

Table 3.5. Christensen criterion requirements [90] [91] .......................................................................... 49

Table 4.1. Comparison of results for various mesh densities in the cross section of the reflector model

Table 4.2. Comparison of results for various mesh densities in the cross section of the reflector model

Table 4.3. Comparison of results for various mesh densities in the cross section of the reflector model

Table 4.4. Comparison of results for various mesh densities in the cross section of the reflector model

Table 4.5. Film coefficient estimations on the vertical faces of the beryllium reflector. .64

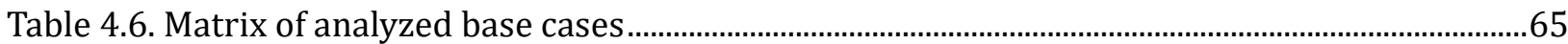

Table 4.7. List of uncertainties in the finite element models of the reflectors .........................................66

Table 5.1. Maximum stress values in reflectors (MPa) with base properties ............................................76

Table 5.2. Ratio between the maximum stresses and local UTS of beryllium material in various models

Table 5.3. Compressive stresses in reflector without beamport hole, with HEU and LEU cores, at 8, 10, and 12 years of hypothetical use (units in $\mathrm{MPa}$ ) 


\section{Introduction}

The U.S. High Performance Research Reactor (USHPRR) Conversion Program, as part of the U.S. National Nuclear Security Administration Office of Material Management and Minimization $\left(\mathrm{M}^{3}\right)$, supports the conversion of nuclear research and test reactors from the use of highly enriched uranium (HEU) to low-enriched uranium (LEU). The University of Missouri Research Reactor $\left(M_{U R R}{ }^{\circledR}\right)$ is one of six USHPRRs, including one critical facility that is engaged in conversion using a high-density LEU fuel that has been proposed for these reactors.

MURR first went critical in 1966. Initially, it was operated at a varying, yet low power level, which was increased to $5 \mathrm{MW}$ within a year of operation. The power was subsequently increased to $10 \mathrm{MW}$ in 1974 and the reactor started operating for three shifts per day; in 1977, it reached the output level of $10 \mathrm{MW}$ for about 150 hours per week, and it has maintained this level and schedule ever since [1].

Many research reactors, including MURR, the Advanced Test Reactor (ATR), and the High Flux Isotope Reactor (HFIR), use beryllium as a moderator and reflector of fast neutrons generated in fission reactions. The MURR beryllium reflector is a cylindrical sleeve located around the reactor pressure vessel at the height of the reactor core. A schematic of the MURR components surrounding the beryllium reflector is shown in Figure 1.1. The basic dimensions of the reflector are shown in Figure 1.2. The MURR reflector is designed with five grooves that are cut along the height of the inner surface of the reflector. The grooves are nominally $0.375 \mathrm{in}$. wide and $0.818 \mathrm{in}$. deep, or about one-third of the total reflector thickness, and hold spacer blocks that ensure a minimum distance between the reflector and the reactor outer pressure vessel [2], [3]. The space must be maintained to ensure free movement of the control blades used for reactivity control and reactor shutdown within this space.

The first MURR beryllium reflector was designed with two beamport holes located near the midheight of the reflector. It failed in May of 1981 at around 27,100 MWd of operation. The reflector fractured into two parts, upper and lower, separated by a crack at the height of the beamport holes, which is schematically illustrated in Figure 1.2. Some small pieces of beryllium fell into the pool following the failure. The failed reflector was replaced later that year with one of an identical design and the same beryllium grade (N-200-A) as the first one. To preclude the likelihood of another failure, this reflector and all subsequent reflectors have been replaced after a predetermined period of use. An operating limit of 26,000 MWd for beryllium was selected, which corresponds approximately to 8 years of operation for the reactor in a core operating at $10 \mathrm{MW}$. The third reflector had only one beamport hole (in the so-called "F" position), which was subsequently removed from the design of the fourth and subsequent reflectors. The replacement schedule for the MURR beryllium reflectors is listed in Table 1.1. The third reflector and all subsequent ones have been built of S-200-FH grade beryllium. No failures of the MURR beryllium reflector have occurred since the failure of the first reflector. It remains to be determined if the lack of failures since 1981 is due to 1) change of beryllium grade, 2) elimination of the beamport holes, 3) controlled operation time of the reflector, or 4) variance in the material properties and surface condition of the reflector. 


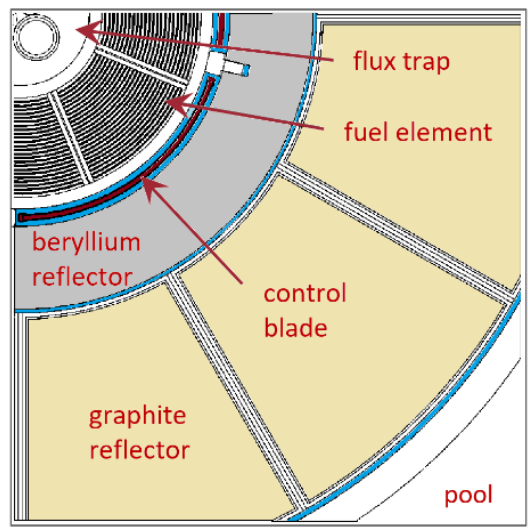

Figure 1.1. Sketch of MURR components in vicinity of beryllium reflector
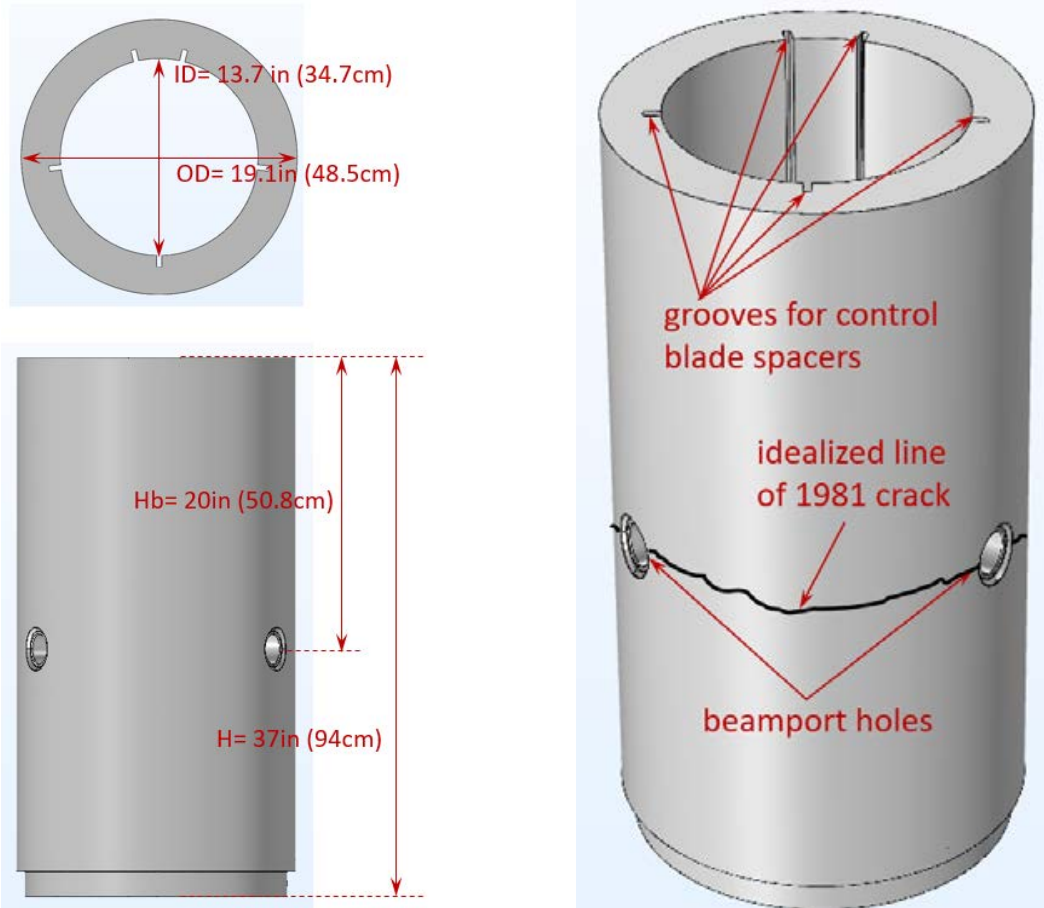

Figure 1.2. MURR beryllium reflector: basic dimensions (left) and schematic of crack location in reflector from 1981 (right)

Table 1.1. Replacement schedule for MURR beryllium reflectors

\begin{tabular}{|l|l|l|l|l|}
\hline$\#$ & Beryllium grade & Number of beamport holes & Replacement date & Operation time \\
\hline $\mathbf{1}$ & N-200-A (failed) & Two & March 1981 & $\sim 27,100 \mathrm{MWd}$ \\
\hline $\mathbf{2}$ & N-200-A & Two & November 1989 & $\sim 26,000 \mathrm{MWd}$ \\
\hline $\mathbf{3}$ & S-200-FH & One in “F” position & September 1997 & $\sim 26,000 \mathrm{MWd}$ \\
\hline $\mathbf{4}$ & S-200-FH & None & January 2006 & $\sim 26,000 \mathrm{MWd}$ \\
\hline $\mathbf{5}$ & S-200-FH & None & March 2014 & $\sim 26,000 \mathrm{MWd}$ \\
\hline $\mathbf{6}$ & S-200-FH & None & Still in operation & Still in operation \\
\hline
\end{tabular}

Studies indicated that for the proposed MURR LEU-fueled core, the steady-state operating power must be increased from $10 \mathrm{MW}$ to $12 \mathrm{MW}$ in order to maintain the same level of neutron flux after conversion at various experimental locations within the reactor [4]. Core safety analyses were 
completed with the new LEU fuel and power uprate and showed similar margins of safety to the HEU core at $10 \mathrm{MW}$ [5]. Subsequent neutronics calculations [6] suggested that the change in the fuel system from HEU to LEU and this power uprate will lead to an $11 \%$ increase in the rate of gas concentration in the beryllium reflector and a $21 \%$ reduction in photon heating. Consequently, it must be determined if the beryllium reflector with the LEU core and power uprate to $12 \mathrm{MW}$ will be able to be used for the same operating period as the current HEU core at $10 \mathrm{MW}$.

During reactor operation, increasing neutron fluence causes degradation of the beryllium material through displacement and transmutation. Displacement damage (measured in terms of displacements per atom or dpa) causes defects in the crystalline structure of the beryllium, leading to hardening and embrittlement [7]. As a result, thermal and mechanical properties of the beryllium worsen, as manifested through reduction of thermal conductivity, fracture toughness, and ductility. Although initial gains in ultimate tensile strength (UTS) and ultimate compressive strength (UCS) are observable for low levels of irradiation, with sufficiently large amounts of irradiation [8], [9], [10], [11], UTS and UCS drop significantly below the levels measured in unirradiated beryllium [8], [12], [13], [14]. Owing to the transmutation of beryllium, helium and tritium gases are produced. These can cause substantial swelling and induce internal stresses [8], [15], [16]. Additional stresses in beryllium reflectors are produced as a result of neutron and photon heating. Thus, the life of the beryllium in reflectors is limited by the thermal stresses: ever-growing stresses induced by transmutation gases, and progressive reduction of material strength to resist these stresses.

The goal of this report is to estimate the impact that conversion of the MURR reactor from HEU to LEU, and the associated power uprate, will have on the life of the beryllium reflector. For that purpose, neutronics calculations were performed to determine the impact of conversion on the production of transmutation gases, the relative damage to beryllium material in terms of average dpa, and the amount of heating produced with HEU and LEU cores. The results of that analysis are presented briefly in Section 2 of this report. A detailed description of this analysis and relevant results are presented more extensively in Appendix A. A literature review was performed to understand the mechanical properties degradation rate as a result of the irradiation process in beryllium material, as well as to propose failure criteria for irradiated beryllium material. The results of that review are presented in Section 3. Structural analysis was performed to estimate the difference in stress levels in the beryllium reflector for the two types of reactor cores as well as for the historical reflector geometry with beamport holes vs. the current design without the holes. The results of that analysis, performed with the COMSOL v. 5.3a software [17], are presented in Sections 4 and 5. Lastly, an attempt to explain the failure of the reflector in 1981 is made, and the relative life of the reflector with the current HEU fuel at $10 \mathrm{MW}$ and with the proposed LEU fuel at $12 \mathrm{MW}$ is calculated and presented in Section 5. The conclusions of this work are presented in Section 6. 


\section{Neutronics Analysis of HEU and LEU Cores}

In order to provide necessary input to the structural analysis of the MURR beryllium reflector, calculations have been completed to predict the spatially distributed neutron flux and the corresponding He-4 gas production, neutron induced damage at microstructure level measured in terms of average number of displacements per atom, dpa after 8 years of simulated operation time, and heating in the reflector, for the HEU and LEU fuel cases. In this analysis, all HEU cases were completed with a core power of $10 \mathrm{MW}$ and LEU cases were completed at $12 \mathrm{MW}$. Predictions of the reflector irradiation conditions have been made using results from MCNP5 v. 1.6 [18]. The MCNP5 models used in the current analysis are the same as those used to support MURR safety analyses for HEU and LEU cores [5], but with the beryllium reflector discretized into many more regions to provide the spatial distribution needed for this work. The MCNP5 analysis does not model material depletion, but the neutron flux and the corresponding He-4 gas production, damage, and heating in the beryllium were predicted assuming a fresh (unirradiated) beryllium reflector, which was determined to yield reasonable predictions over the life of the reflector given the low depletion of the reflector over its anticipated lifetime. All predictions reported in this section were performed for 8 years of operation, which corresponds to the current replacement schedule at MURR. A detailed description of this analysis is provided in Appendix A. In this section, only the most relevant results are presented.

\subsection{Neutron Flux}

Results relevant to the MURR beryllium reflector lifetime analysis were obtained by partitioning the incident neutron flux into detailed spatial and energy discretizations in MCNP5. The reflector was segmented for tally purposes into 28 radial zones and 45 axial zones. The zones were of non-uniform size, with the smallest zones located in the middle of the height of the reflector and near the inner surface of the reflector. The neutron flux in each zone was tallied for each of 15 energy bins. Segmentation details are provided in Appendix A.

Figure 2.1 (left) presents the spatial distribution of the total neutron flux in the reflector for the case with the HEU core. The neutron flux decreases almost linearly with penetration into the reflector, and is highest near the core midplane. Figure 2.1 (right) shows the flux of neutrons with $\mathrm{E}>1.0 \mathrm{MeV}$ in the reflector converted to fluence at nominally 8 years of operation. The decrease in rate of the fluence of fast neutrons is quite sharp and significantly more nonlinear, which indicates that there will be likely a high localization of the microstructure defects closer to the inner surface of the reflector. This pattern occurs because the neutron flux spectrum becomes increasingly more thermalized from elastic scattering within the beryllium. Figure 2.2 shows similar plots for the case with the LEU core. The maximum total neutron flux is about 3\% higher in the case of the LEU core. The maximum fluence of fast neutrons with $\mathrm{E}>1.0 \mathrm{MeV}$ is about $9 \%$ higher for the case with the LEU core. The maximum fluence of fast neutrons with $\mathrm{E}>0.1 \mathrm{MeV}$ is about $10 \%$ higher for the case with LEU core. Figure 2.3 compares the fluence through the thickness of the reflector at mid-height for HEU and LEU cores. 

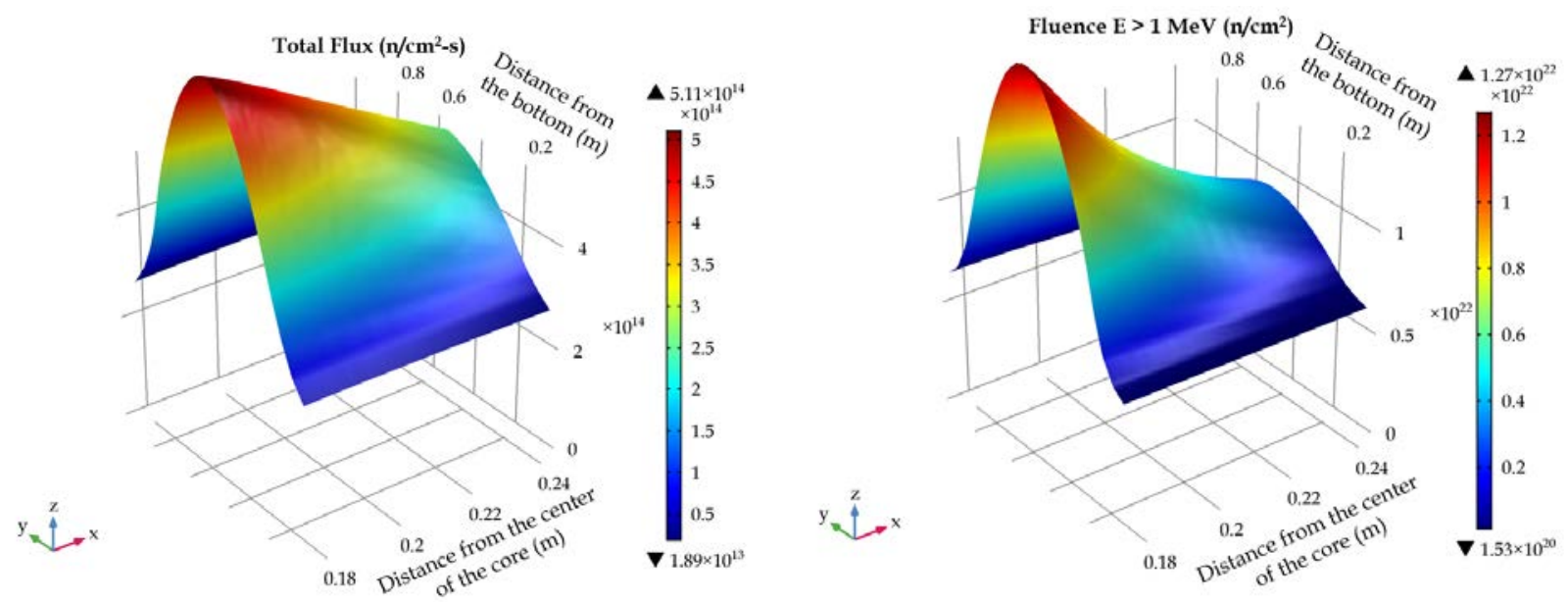

Figure 2.1. Distribution of total flux (left) and fast fluence ( $\mathrm{E}>1 \mathrm{MeV}$ ) after 8 years (right) in beryllium reflector with HEU core
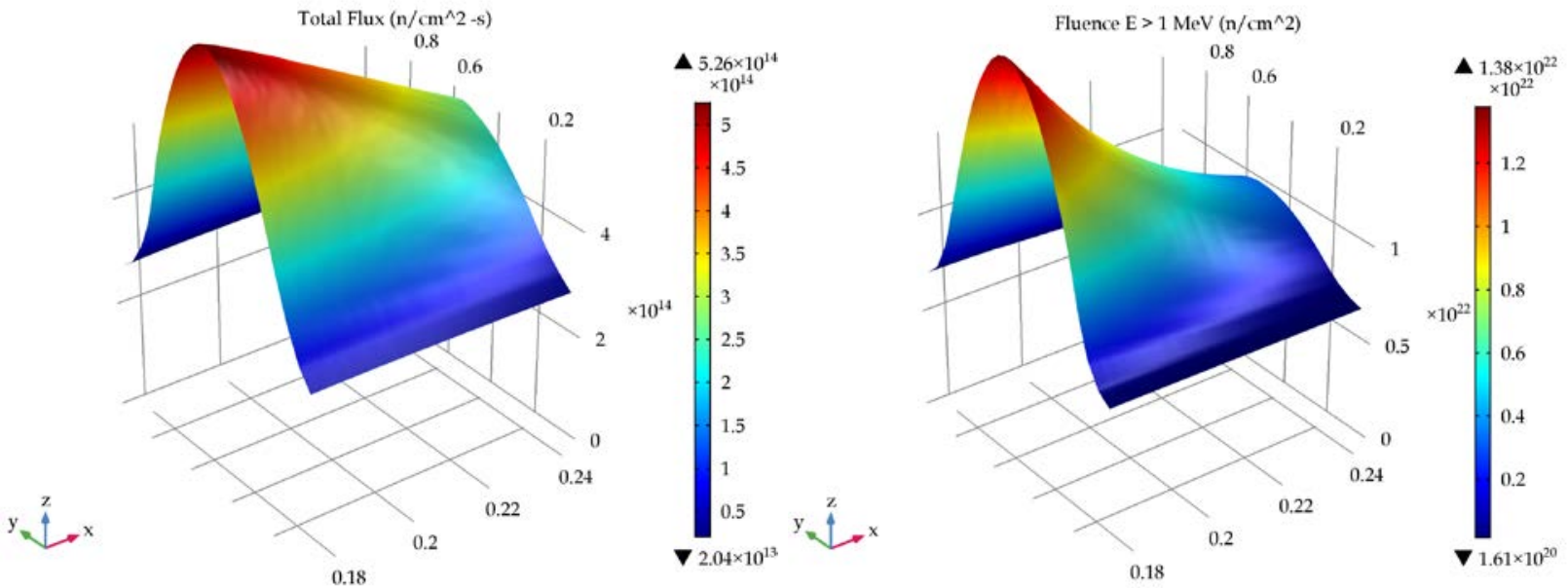

Figure 2.2. Distribution of total flux (left) and fast fluence ( $\mathrm{E}>1 \mathrm{MeV}$ ) after 8 years (right) in beryllium reflector with LEU core 


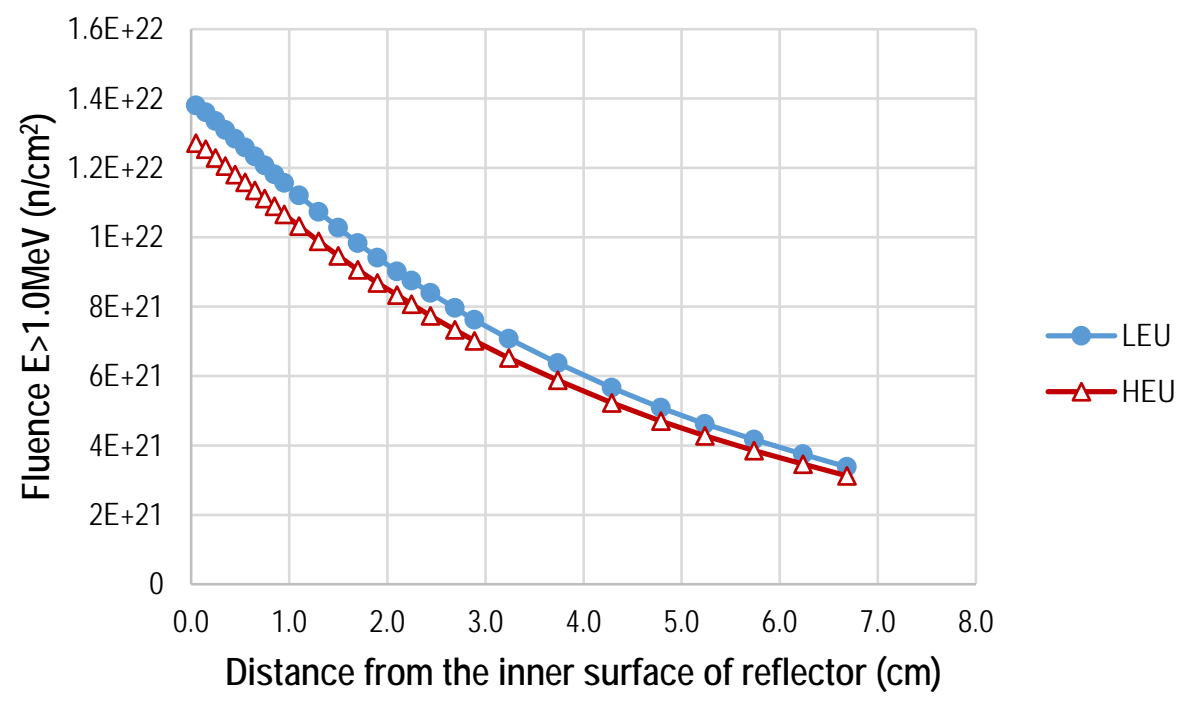

Figure 2.3. Fast fluence $(\mathrm{E}>1.0 \mathrm{MeV})$ through the reflector at mid-height after 8 years with HEU and LEU cores

\subsection{Helium Production}

The neutron and photon reactions considered most relevant for the analysis of the MURR reflector depletion are as follows:

$$
\begin{aligned}
& \text { Be- } 9+\mathrm{n} \rightarrow \mathrm{Be}-8+2 \mathrm{n} \text { (n,2n reaction) Be- } 8 \rightarrow 2 \mathrm{He}-4 \text { (short decay) } \\
& \text { Be- } 9+n \rightarrow \text { He- } 6+\text { He- } 4 \text { (n, } \alpha \text { reaction) He- } 6 \rightarrow \text { Li-6 (short decay) } \\
& \mathrm{Li}-6+\mathrm{n} \rightarrow \mathrm{He}-4+\mathrm{H}-3 \text { (n,T reaction) } \\
& \text { Be- } 9+\gamma \rightarrow \mathrm{n}+2 \mathrm{He}-4(\gamma, 2 \alpha \text { reaction })
\end{aligned}
$$

Helium-4 is produced primarily from three reactions: 1$)(n, 2 n)$ reactions in $B e-9,2)(n, \alpha)$ reactions in Be-9, and 3) (n,T) reactions in Li-6 as a by-product of the beryllium depletion. Note that the (n,2n) and $(n, \alpha)$ reactions in Be-9 are threshold reactions that only occur with high-energy neutrons. The MCNP5 analysis shows that there is also a very small amount of He-4 produced from a photoninduced $(\gamma, 2 \alpha)$ reaction in the beryllium. It should be noted that the Be-9 $(n, 2 n)$ reaction produces two He-4 atoms per reaction, compared with a yield of 1 for the Be-9 $(n, \alpha)$ and Li- $6(n, T)$ reactions. Consequently, the Be-9 $(\mathrm{n}, 2 \mathrm{n})$ reaction dominates the total helium production in the reflector. More detailed descriptions of these reactions, assumptions, and results of the analysis are included in Appendix A.

Figure 2.4 and Figure 2.5 show the He-4 distribution in the reflector with the HEU and LEU cores after 8 years ( 7.2 full-power years) as predicted by MCNP5. The left-hand panel shows the distribution of He-4 in a vertical (axisymmetric) section of the reflector. The right-hand panel of each figure represents the distribution of He- 4 in the COMSOL model after import of the data.

The majority of the helium production $(>80 \%)$ is from the Be- $9(n, 2 n)$ reaction. The character of the distribution within the MURR beryllium reflector is similar to the fast fluence distribution as shown in Figure 2.1 and Figure 2.2 for the HEU and LEU cores, respectively (rather than to the total 
flux/fluence distribution). This is because the $(n, 2 n)$ reaction in Be-9 is a threshold reaction and all $(n, 2 n)$ reactions in Be-9 occur for neutrons with $\mathrm{E}>1.0 \mathrm{MeV}$.
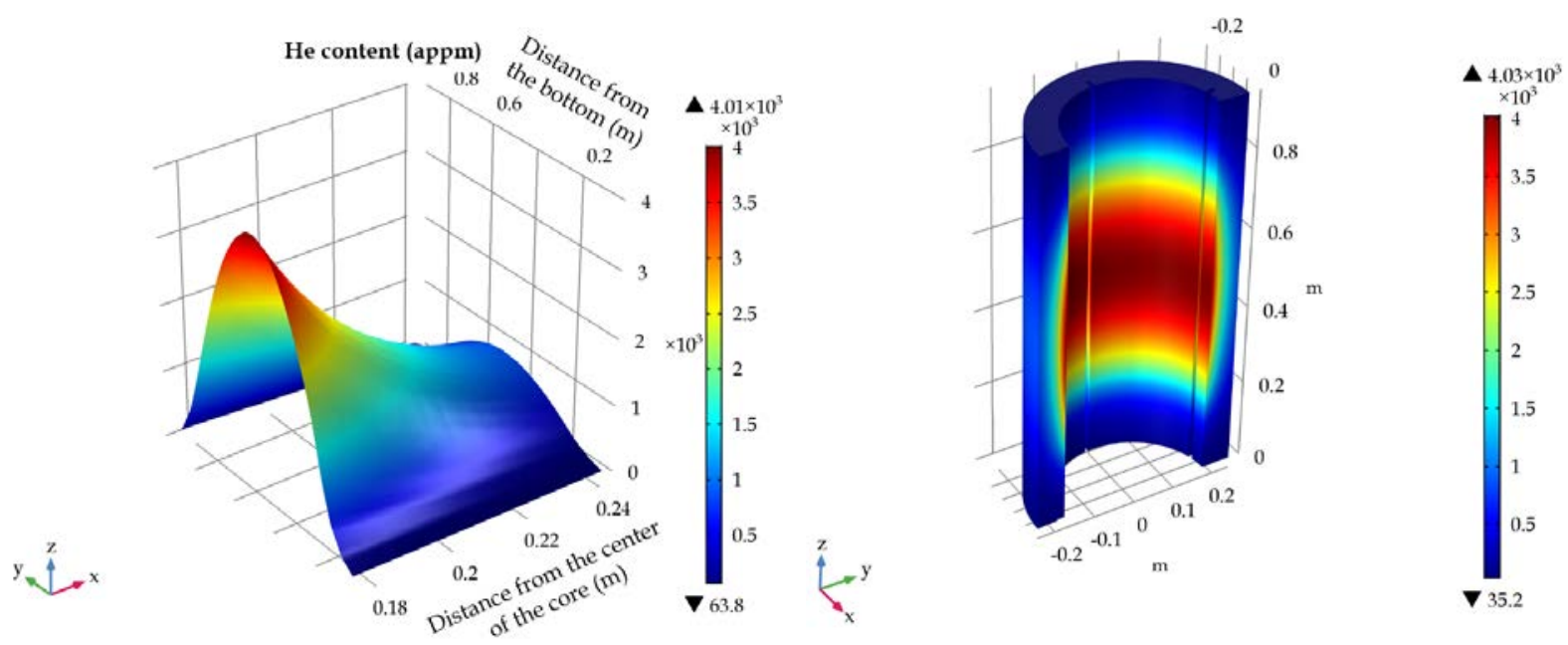

Figure 2.4. Distribution of He-4 content in beryllium reflector at 8 years with HEU core (in units of appm). Data imported to COMSOL (left); data extrapolated on the beryllium reflector model (right).
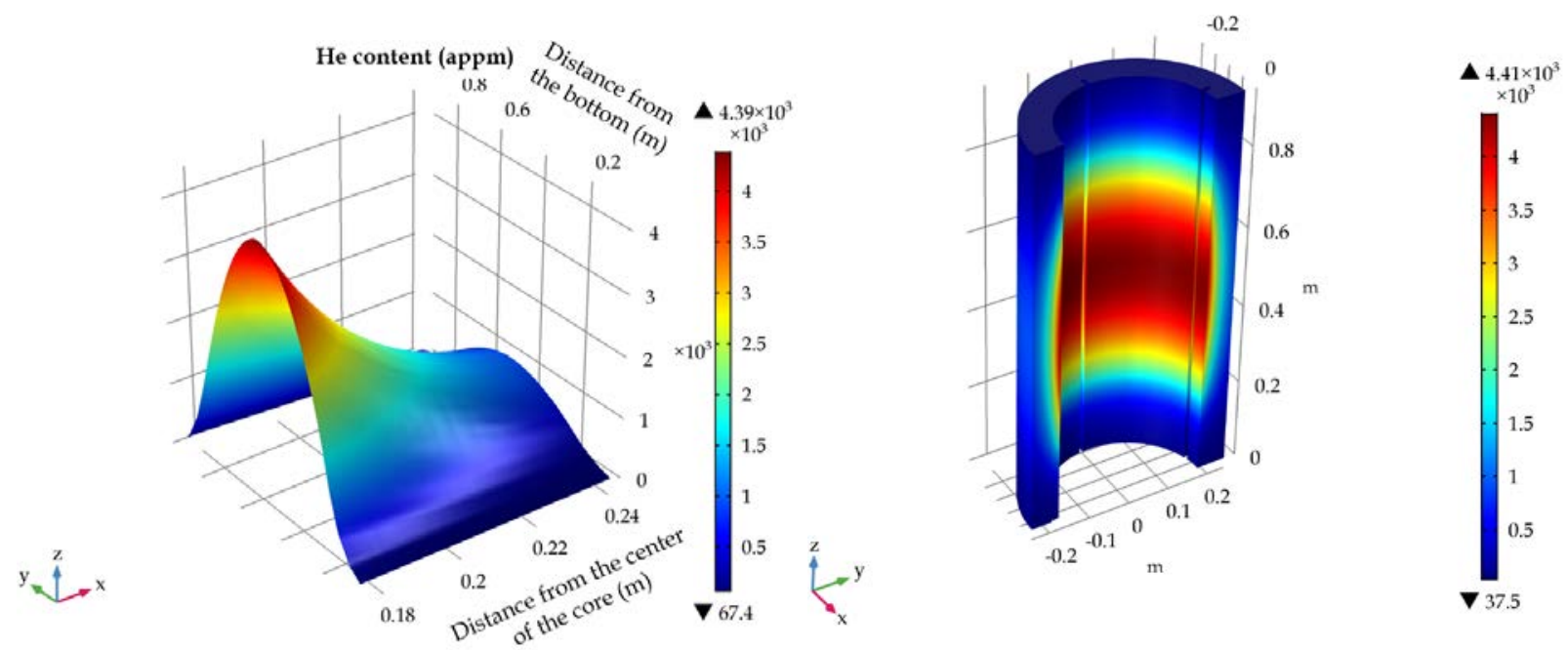

Figure 2.5. Distribution of He-4 content in beryllium reflector at 8 years with LEU core (in units of appm). Data imported to COMSOL (left); data extrapolated on the beryllium reflector model (right).

The analysis estimates that after 8 years of operation, the peak helium concentration for the HEU core under these conditions ( 7.2 full-power years) is 4,005 appm, located at the inner surface of the reflector at the core midplane. The corresponding peak helium concentration for the LEU core is 4,377 appm at the same location. The peak value, as well as the average amount of He-4 in the beryllium reflector, is thus predicted to be about $9 \%$ higher in the reflector with the LEU core relative to the HEU core (see Figure 2.6). The content of He-4 is a primary contributor to the swelling and consequently to swelling-induced stresses. Thus, swelling-induced stresses are predicted to increase at a higher rate in the beryllium reflector with LEU fuel than the one with HEU fuel. 
Note that the distribution profiles on the right side of Figure 2.4 and Figure 2.5 have slightly higher maximum values than the profiles of the MCNP5 results presented on the left. The MCNP5 results are plotted at the center of each tally cell in the model. The COMSOL-generated profiles on the right are extrapolated to the edge of the surface elements. This extrapolation applies to all imports of data from the MCNP5 models, including He-4 content, damage (dpa), and heating profiles. The extrapolation results in maximum values in the COMSOL models that are only about $1 \%$ greater than the MCNP5 predictions and thus is seen to have a negligible effect.

Also, for the structural analysis presented in this report, azimuthally averaged results of neutronics analysis were used. Appendix A presents more refined results, in which localized distributions of the He- 4 at the bottom of the grooves and on the outer surface of the reflector in line with the grooves are reported. The results showed that the localized He- 4 concentration at these locations can be about $10 \%$ higher than the azimuthally averaged data estimates. The difference is only about $2 \%$ on the outer surface of the reflector if compared to the azimuthally averaged results. This part of the neutronics analysis was performed only after the FE analysis in COMSOL was done on the basis of the azimuthally averaged inputs. While it may slightly increase the level of stresses on the outer surface of the reflector, the effect will show a similar magnitude for both the HEU and LEU cases. Thus, the conclusions drawn from the analysis with azimuthally averaged inputs will remain valid. The localized damage (dpa) on the outer surface behind the grooves may be up to $6 \%$ higher than the azimuthally averaged results. This effect has been included in the final life estimation of the MURR beryllium reflector.

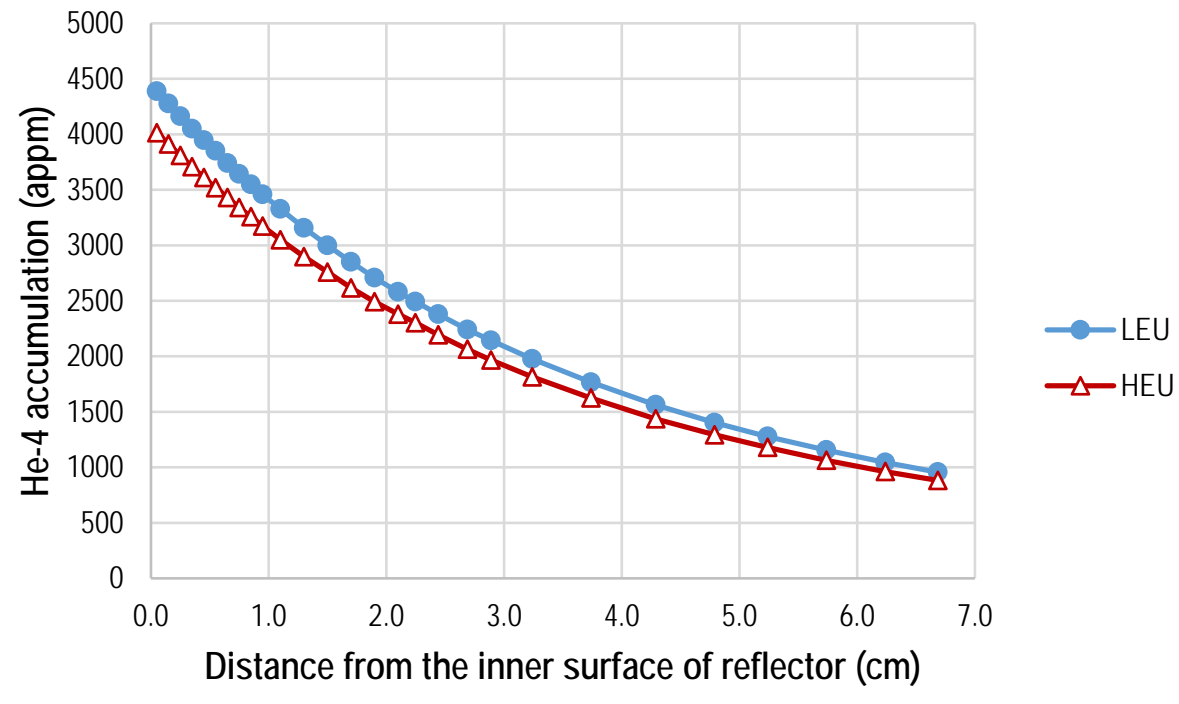

Figure 2.6. He-4 concentration through the reflector at mid-height with HEU and LEU cores after 8 years

\subsection{Irradiation Damage (Displacements per Atom)}

Figure 2.7 and Figure 2.8 show the distribution of damage, in terms of dpa, in the beryllium reflector at 8 years of operation (7.2 full-power years) for HEU and LEU cores, respectively. The irradiation damage was estimated in the MCNP5 analysis using a flux multiplier tally with the MT=444 reaction in Be-9 (ENDF/B-VII cross section library 4009.70c). As the MCNP5 calculations indicate, the majority of the damage from atomic displacement occurs from neutrons with energies greater than $100 \mathrm{keV}$, although about $20 \%$ of the reflector damage does occur from neutrons with energies $<100 \mathrm{keV}$. It is 
thus observed that damage in beryllium is caused by both epithermal and fast neutrons. As a result, the distribution of the damage through the thickness of the reflector, like the total flux distribution, exhibits an almost linear decrease with penetration in the reflector (see Figure 2.9) as compared to the nonlinear shape with reflector penetration for the fast-neutron flux (see Figure 2.1 and Figure 2.2).

The peak damage present after 8 years on the inner surface of the reflector near the mid-height for the HEU core is $15.7 \mathrm{dpa}$, while it is $17.3 \mathrm{dpa}$ for the LEU core (see Figure 2.9). Thus, more degradation of strength and thermal conductivity is expected in the beryllium reflector with the LEU core after 8 years of operation than with the HEU core.

Degradation of properties in beryllium is typically a nonlinear function of damage. As a result, the relative degradation of the material properties for the reflectors with HEU and LEU cores that experience differing amounts of damage cannot be determined without prior testing of samples under relevant irradiation conditions. Efforts to characterize the degradation of properties due to neutron damage on the basis of available data from the literature are described in Section 3.
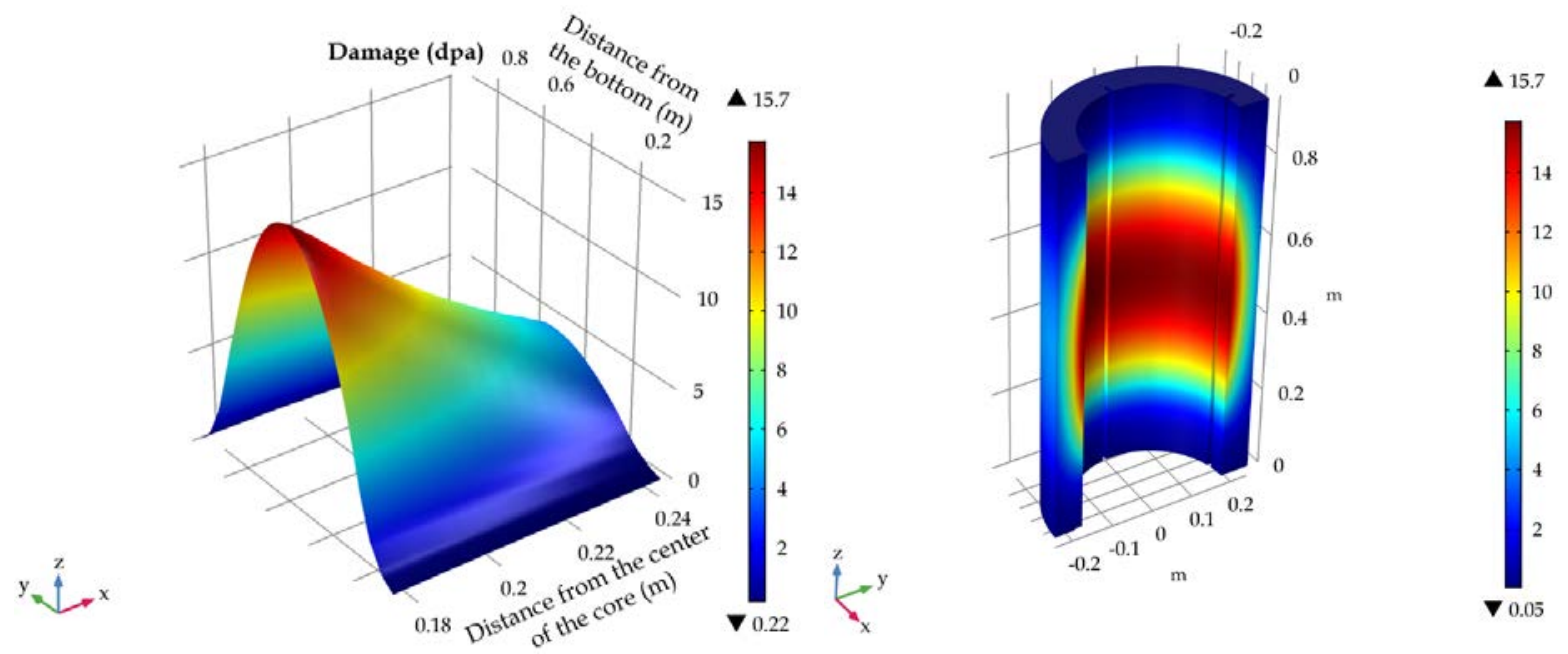

Figure 2.7. Distribution of damage in beryllium reflector with HEU core at 8 years of operation (in units of dpa). Data imported to COMSOL (left); data extrapolated on the beryllium reflector model (right). 

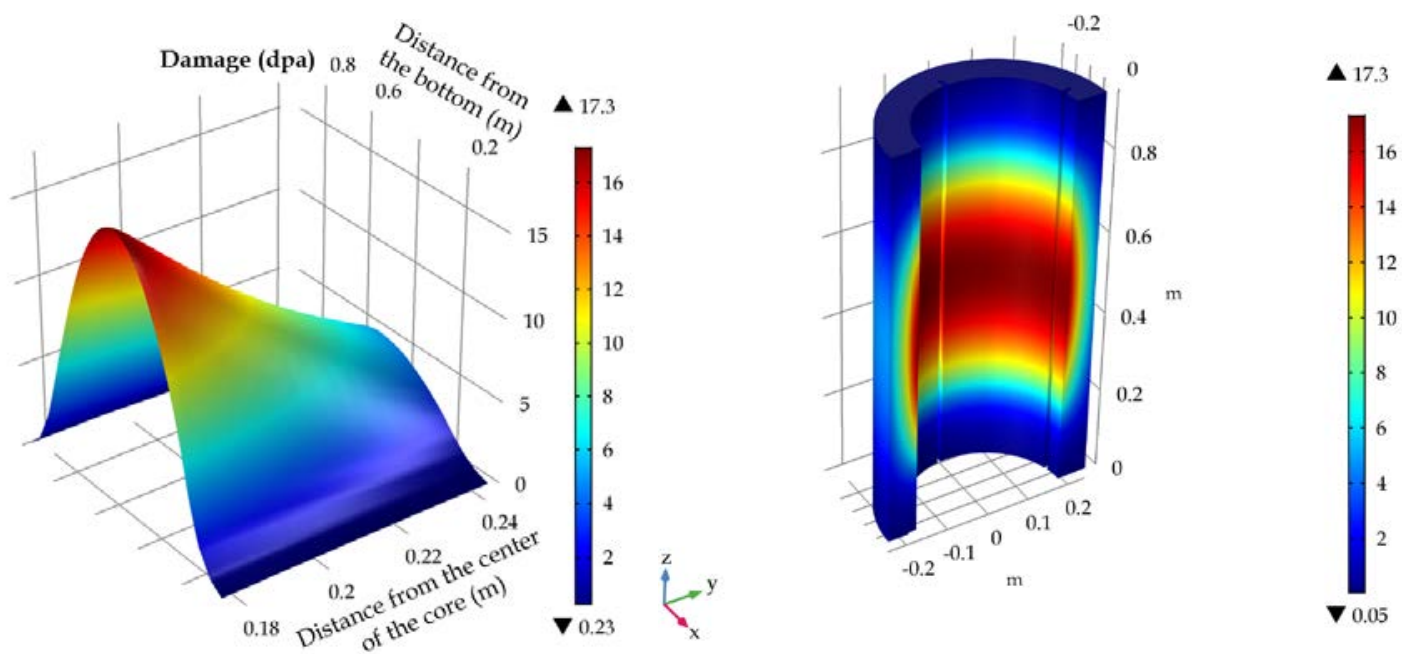

Figure 2.8. Distribution of damage in beryllium reflector with LEU core at 8 years of operation (in units of dpa). Data imported to COMSOL (left); data extrapolated on the beryllium reflector model (right).

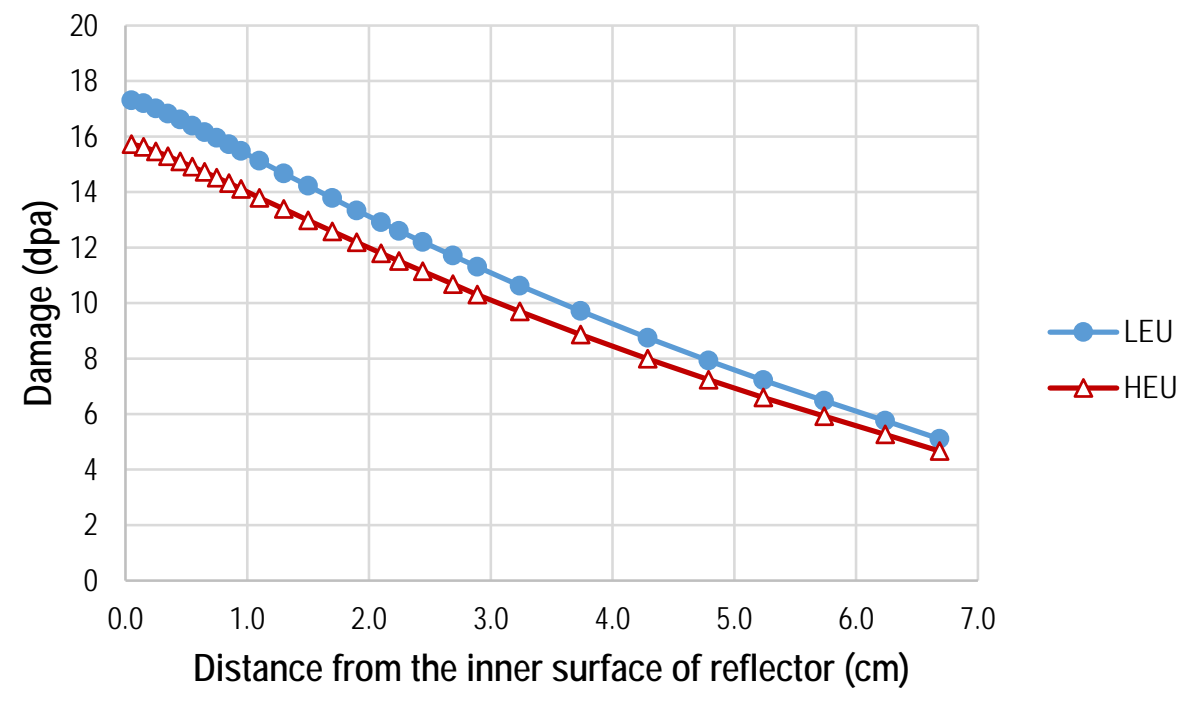

Figure 2.9. Damage through the reflector at mid-height for HEU and LEU cores

\subsection{Neutron and Photon Heat Generation}

During operation, the reflector will be heated by energy deposition from neutron and photon interactions with the beryllium, which induces thermal stresses in the beryllium reflector. While the stresses induced by swelling increase almost linearly with time (because of a linear increase of He-4 gas concentration with operation time), the stresses from heating reach maximum values almost instantaneously after the start of reactor operations. Moreover, they will have a cyclic character because of the weekly MURR operating/refueling schedule [5].

In the MCNP5 calculations it is assumed that the overall internal heat generation in the beryllium reflector will be the sum of heat generation caused by neutrons, prompt photons, and delayed photons. A detailed description of the assumptions for this analysis and comparison to previously obtained results [6] is presented in Appendix A. 
Prompt and delayed photon interactions account for about $80 \%$ of the total heating in the reflector. Figure 2.10 and Figure 2.11 show the distribution of photon heating in the beryllium reflector for HEU and LEU cores, respectively. The photon heating in the reflector is $18 \%$ lower with the LEU core operating at $12 \mathrm{MW}$, relative to the HEU core operating at $10 \mathrm{MW}$. On the other hand, neutron heating is about $10 \%$ greater in the reflector with the LEU core relative to the HEU core. Figure 2.12 shows the total heating with penetration in the reflector with HEU and LEU cores. The peak energy deposition in the reflector with the LEU core and power uprate is predicted to be $5.11 \mathrm{~W} / \mathrm{cm}^{3}$, which is $13.4 \%$ less than for the current operation with the HEU core $\left(5.91 \mathrm{~W} / \mathrm{cm}^{3}\right)$.

In 2013, a study was performed on the impact of HFIR's HEU-to-LEU conversion on berylliumreflector degradation [19]. The assumption for the conversion of HFIR included an $18 \%$ increase in total core power (from $85 \mathrm{MW}$ to $100 \mathrm{MW}$ ). The analysis found that in HFIR, gas production in the beryllium with the LEU core and the power uprate would increase by $\sim 12 \%$, while the total heating rate in the beryllium reflector would decrease by $\sim 20 \%$. These values are consistent with the trends found in the current study for the MURR beryllium reflector with a $20 \%$ power uprate following conversion.

As mentioned earlier, the total stresses in the beryllium reflector are a result of internal heat generation and He-4-induced swelling. For the LEU core and $12 \mathrm{MW}$ power, the stresses due to swelling and neutron heating will be respectively greater than with the HEU core at $10 \mathrm{MW}$, while stresses due to photon heating will be lower for the LEU core. Structural analysis described in Section 4 will be performed to assess the relative change in total stress following conversion from HEU to LEU.
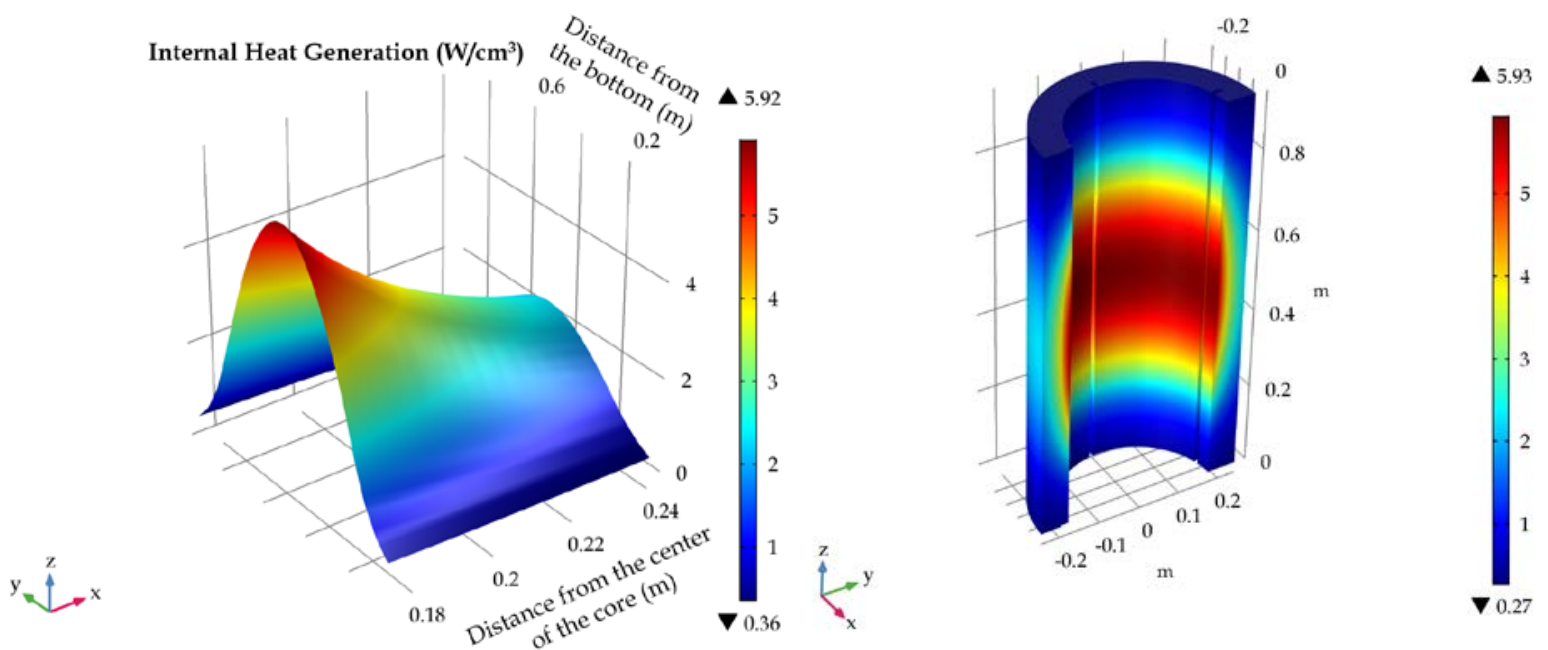

Figure 2.10. Distribution of internal photon heating in the beryllium reflector with the HEU core $\left(\mathrm{W} / \mathrm{cm}^{3}\right)$. Data imported to COMSOL (left); data extrapolated on the beryllium reflector model (right). 

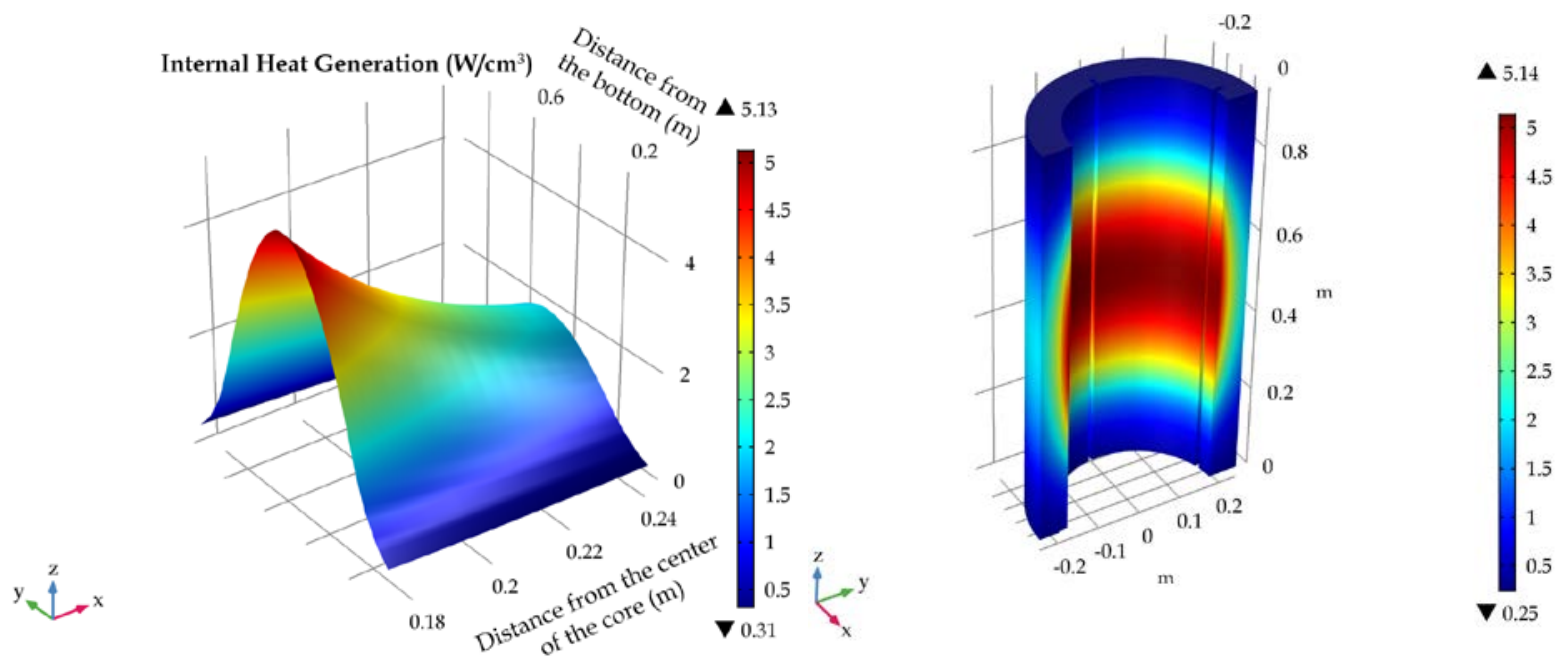

Figure 2.11. Distribution of photon heating in beryllium reflector with the LEU core $\left(\mathrm{W} / \mathrm{cm}^{3}\right)$. Data imported to COMSOL (left); data extrapolated on the beryllium reflector model (right).

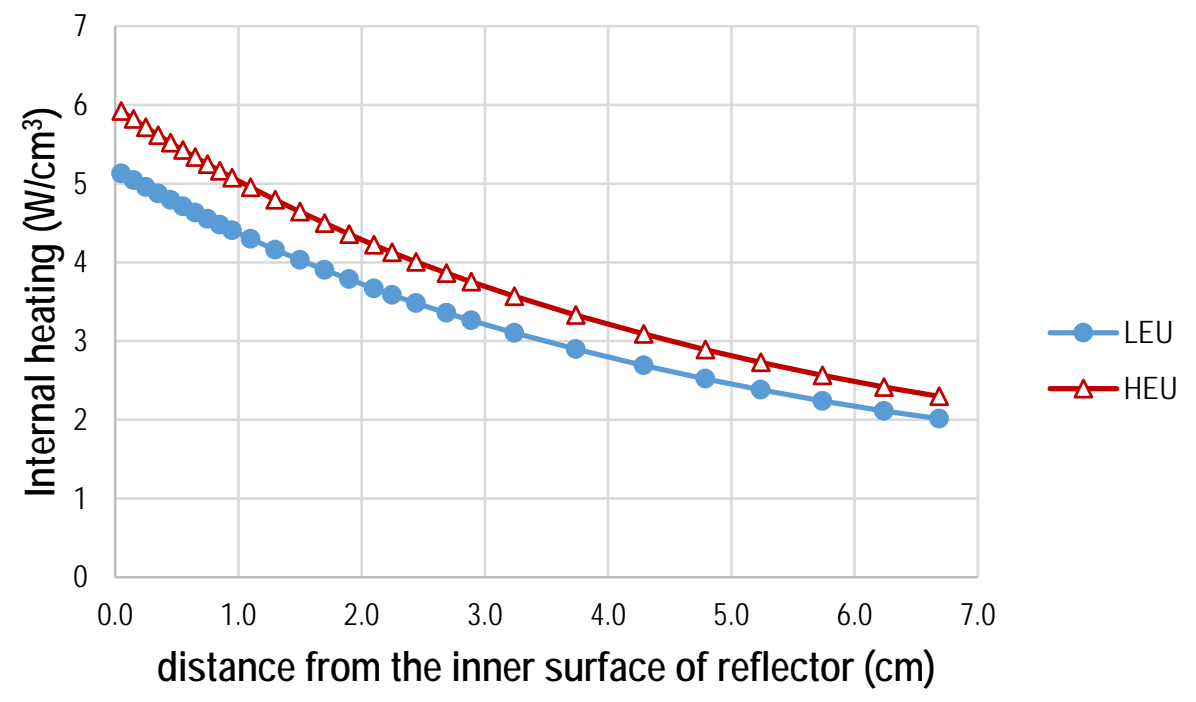

Figure 2.12. Internal heating through the reflector at mid-height for HEU and LEU cores 


\section{Beryllium Material Modeling}

It is not the intent of this report to list all the thermomechanical properties of beryllium and their dependence on manufacturing (chemical composition, method of production) or environmental factors (temperature, irradiation levels). The primary goal is, rather, to collect the data needed to characterize the properties of the grades used historically and currently by MURR for beryllium reflectors, primarily under conditions experienced by the reflector in the MURR reactor, in order to build the FE model needed to predict the life of MURR beryllium reflectors. For a broader description of thermomechanical properties of various beryllium grades, the reader should refer to other sources, such as [20], [21], [22], and [23].

\subsection{Material Properties of Fresh (Unirradiated) Beryllium}

The historical and the currently most popular structural grades of beryllium are produced through various methods of powder metallurgy. In the late 1950s, when the MURR was designed, a nuclear grade of beryllium was produced by the Brush Beryllium Company (the predecessor of Materion). The first two MURR reflectors were built at the same time from currently obsolete nuclear grade N200-A. It was produced from hot pressing of the powder that was produced from vacuum-cast ingots. This method of production led to anisotropy in the mechanical properties and low ductility. Throughout the last 60 years or so, the structural grade S-200, the most popular grade of beryllium produced by Brush Beryllium, has been produced by hot extruding. New generations of S-200 were designated by consecutive letters of the alphabet, with S-200-C available in the late 1950s and through the 1960s, and S-200-F available currently.

The differences in the thermomechanical properties of various grades of beryllium are attributable not only to the chemical composition and the method of consolidation of the powder, but also to the source and the shape of the individual grains in the powder. The initial structural grades of beryllium were produced from mixes of virgin and scrap beryllium powders and therefore had a higher content of impurities and non-uniform properties. S-200-E no longer contained recycled powder, and its consistency in strength properties was improved [24] over the prior grades. The production of beryllium begins with chipping of vacuum-cast beryllium ingots [25]. The powder for S-200-E was produced by grinding beryllium chips between two beryllium disks, a method called attrition grinding, producing flake-shaped particles [20]. Powder production for S-200-F, a modification of S200-E, uses an impact-grinding technique, which results in more isotropic properties of the final product [25]. S-200-F is currently one of the most popular grades of beryllium. It is used for production of reflectors installed at ATR and the Japan Materials Testing Reactor (JMTR) [26], among others.

The beryllium grade used currently in MURR is designated as S-200-FH. It is produced through the consolidation of beryllium powder by a hot isostatic pressing (HIP) process introduced at Materion in 1985 [27]. The previous grades, including S-200-F, used a vacuum hot-pressing (VHP) method for powder consolidation. Despite many similarities, there are notable differences between the S-200-F and S-200-FH grades. The average grain size, which has a significant impact on the strength properties of beryllium, is lower for S-200-FH than for S-200-F (maximum of $12 \mu \mathrm{m}$ vs. $20 \mu \mathrm{m}$ ). Usually, the smaller the grain size, the greater the strength and ductility of beryllium [23]. Produced by HIP, the S-200-FH grade is much more isotropic and has more desirable mechanical properties than the traditional vacuum hot-pressed grades [28]. However, in the absence of some properties for $\mathrm{S}-200-\mathrm{FH}$ grade in the literature, the properties of S-200-F grade may be used as an indication of trends and conservative estimations for S-200-FH. 
Thermomechanical properties of S-200-FH grade, together with the properties of S-200-F and N-200A grades produced by Materion, are gathered in Table 3.1. These data are primarily sourced from Materion [26], [29], [25], [30], [31], [32]. It is worth noting that the fabricators usually guarantee only the minimum (or maximum where applicable) values required for a specific grade, which are not a statistical measure of average properties. For this reason, any calculations based on the minimum (or maximum where conservative, like flaw-related parameters) values of thermomechanical properties may be overly conservative, as they most likely represent values of $\geq 3 \sigma$ below the mean characteristics. The properties in Table 3-1 are based on the specification sheets, as well as the statistical data whenever they were available.

S-200-FH is the strongest, most isotropic and most ductile of the grades considered here. The N-200A grade had a minimum yield strength and minimum UTS notably lower than the equivalent limits for the S-200-F and S-200-FH grades. The literature review did not reveal any statistical evaluations of the thermomechanical properties of $\mathrm{N}-200-\mathrm{A}$ grade. Thus, the calculations for the first, failed reflector are limited to the minimum guaranteed strength properties provided in the specification sheet [32]. 
Table 3.1. Properties of fresh beryllium produced by Materion

\begin{tabular}{|c|c|c|c|}
\hline Material Property & S-200-FH [26], [30] & S-200-F [26], [31] & N-200-A [32] \\
\hline Method of production & Hot isostatic pressed & Vacuum hot pressed & Hot pressed \\
\hline Density, g/cm ${ }^{3}$ & 1.85 & 1.85 & 1.84 \\
\hline Beryllium assay, \% min & 98.5 & 98.5 & 98.0 \\
\hline Beryllium oxide, \% max & 1.5 & 1.5 & 2.0 \\
\hline Average grain size, $\mu \mathrm{m}, \max$ & 12 & 20 & -- \\
\hline Average grain size, $\mu \mathrm{m}$ & $5.3 \pm 3.3[33]$ & $7.1 \pm 4.1[33]$ & -- \\
\hline Young's modulus in tension, GPa & $\begin{array}{c}303 \\
307.7[34]\end{array}$ & $\begin{array}{c}290 \\
310[35] \\
\text { L } 310.9 \pm 6.9[25] \\
\text { T } 309.6 \pm 4.8[25]\end{array}$ & $303.4[36]^{* *}$ \\
\hline Poisson's ratio & $0.04[34]$ & $\begin{array}{c}0.07[37] \\
0.058-0.108[25] \\
\end{array}$ & $0.024[36]^{* *}$ \\
\hline $\begin{array}{l}\text { Minimum yield strength in tension } \\
(0.2 \% \text { offset }), \mathrm{MPa}\end{array}$ & 296 & 241.3 & 206.8 \\
\hline $\begin{array}{l}\text { Typical or measured yield strength in } \\
\text { tension, } \mathrm{MPa}\end{array}$ & $\begin{array}{c}351.6[38] \\
\text { L } 343 \text { std dev 16.7 [25] } \\
\text { T } 346 \text { std dev 16.7 [25] } \\
\text { (327 samples) } 360.66 \text { std dev 14.53 [39] }\end{array}$ & $\begin{array}{l}\text { L 261.3; T } 258.6[38] \\
\text { L 263.4 } \pm 3.4[23] \\
\text { T } 262.0 \pm 0.7[23]\end{array}$ & -- \\
\hline $\begin{array}{l}\text { Minimum ultimate tensile strength, } \\
\mathrm{MPa}\end{array}$ & 414.0 & 324.0 & 275.8 \\
\hline Ultimate tensile strength, MPa & $\begin{array}{c}441.3[38] \\
\text { (67 samples) L } 438 \text { std dev } 9.7[25] \\
\text { (66 samples) T } 447 \text { std dev } 10.8[25] \\
\text { (147 samples) L 454.16 std dev } 13.14[39] \\
\text { (139 samples) L 455.12 std dev } 12.71[39] \\
\text { (180 samples) LT\&T 466.43 std dev } 12.25[39]\end{array}$ & $\begin{array}{l}\text { L } 377.1 \text { T } 407.5[38] \\
\text { L } 372 \text { T } 393[25] \\
\text { L } 382.0 \pm 2.1[23] \\
\text { T } 407.5 \pm 4.8[23]\end{array}$ & -- \\
\hline Elongation, \%, min & 3.0 & 2.0 & 1.0 \\
\hline Elongation at failure, $\%$ & $\begin{array}{c}\text { (67 samples) L } 4.6 \text { std dev } 0.56[25] \\
\text { (66 samples) T } 5.3 \text { std dev } 0.61[25] \\
\text { (147 samples) L } 4.34 \text { std dev } 0.60[39] \\
\text { (139 samples) L } 4.34 \text { std dev } 0.61[39] \\
\text { (180 samples) LT\&T } 5.08 \text { std dev } 0.76[39]\end{array}$ & $\begin{array}{l}\text { L } 4.0 \text { T } 6.0[25] \\
\text { L } 3.4 \pm 0.3[23] \\
\text { T } 6.1 \pm 0.5[23]\end{array}$ & -- \\
\hline $\begin{array}{l}\text { Young's modulus in compression, } \\
\text { GPa }\end{array}$ & -- & -- & $\begin{array}{c}305^{*}[36] \\
304.7^{* *}[36]\end{array}$ \\
\hline Yield strength in compression, $\mathrm{MPa}$ & $\begin{array}{c}\text { Typically } 10 \% \text { higher than the tensile yield } \\
\text { strength [29] }\end{array}$ & $\begin{array}{l}\text { Typically } 10 \% \text { higher than the tensile yield } \\
\text { strength, } 283[29] \\
\text { L } 279.9 \pm 4.8[40] \\
\text { T } 271.0 \pm 9.0[40]\end{array}$ & $286.1^{* *}[36]$ \\
\hline
\end{tabular}


ANL/RTR/TM-20/1

\begin{tabular}{|c|c|c|c|}
\hline Material Property & S-200-FH [26], [30] & S-200-F [26], [31] & N-200-A [32] \\
\hline Ultimate compressive strength, MPa & (5 samples) $1600-1900$ & -- & $1322^{*}[41]$ \\
\hline Total deformation, $\%$ & (5 samples) $32-38$ & -- & $30.1^{*}[41]$ \\
\hline Fracture toughness, MPa-m ${ }^{1 / 2}$ & $\begin{array}{l}\text { (8 samples) mean } 11.3 \pm 0.5[42] \\
\text { (3 samples) L-T } 9.07 \text { std dev } 0.41 \text { [25] } \\
\text { (3 samples) T-L } 9.19 \text { std dev } 0.32 \text { [25] }\end{array}$ & $\begin{array}{c}\text { L } 10.62 \text { T } 12.31 \text { [43] } \\
\text { (3 samples) L-T 9.5 std dev } 0.28 \text { [25] } \\
\text { (4 samples) T-L } 10.9 \text { std dev } 0.38 \text { [25] }\end{array}$ & -- \\
\hline Thermal conductivity, W/m-K & 216 & $\begin{array}{c}216 \\
204[25]\end{array}$ & $\sim 180[25]$ \\
\hline Heat capacity, J/g-K & 1.95 & 1.95 & -- \\
\hline $\begin{array}{l}\text { Coefficient of thermal expansion, } \\
\mathrm{ppm} / \mathrm{K}\end{array}$ & $\begin{array}{c}11.3 \\
11.6[44]\end{array}$ & $\begin{array}{c}11.3 \\
\text { L } 11.39 \mathrm{~T} 111.57 \mathrm{~T} 211.45[40]\end{array}$ & -- \\
\hline
\end{tabular}

*Property of QMV vacuum hot-pressed beryllium without exact specification of the grade, which could denote one of the S- or N- historical grades. (QMV was a brand name for a vacuum hot-pressed beryllium produced in the 1950s through 1970s.)

**Typical property of QMV beryllium produced by Brush Wellman in the 1950s. 


\subsection{Effect of Temperature on Material Properties of Fresh (Unirradiated) Beryllium}

On the basis of the current analysis, the temperature of beryllium during its operation in MURR will fall in the range of 60 to $100^{\circ} \mathrm{C}$. Although this is a rather small range of temperatures, some of the material properties will be affected by it. Not all of the properties of the S-200-FH grade have been tested and documented for all temperatures of interest. In cases where such data are lacking, dependency of properties of similar grades (including S-200-F) of beryllium will be presented in this section. This information is still useful for understanding the trends in the change of properties related to the structural performance of the beryllium reflector.

It must be noted that the thermal effects presented in this section cannot be superimposed on the irradiation effects presented in the following section. The data gathered in this section are only used to define the starting values for the properties degradation under irradiation.

Figure 3.1 (left) presents stress-strain curves from tensile tests of S-200-F grade at three temperatures, with the lowest being room temperature (RT) (digitized from [20]). Reduction in the initial slope of the curve (Young's modulus), yield strength, and UTS with increasing temperature can be noted. Figure 3.1 (right) presents stress-strain curves for five samples of S-200-FH grade tested in compression at RT (digitized from [45]). No compressive strength data at elevated temperatures were found in the literature, but similar reduction of strength as observed in tension is expected.
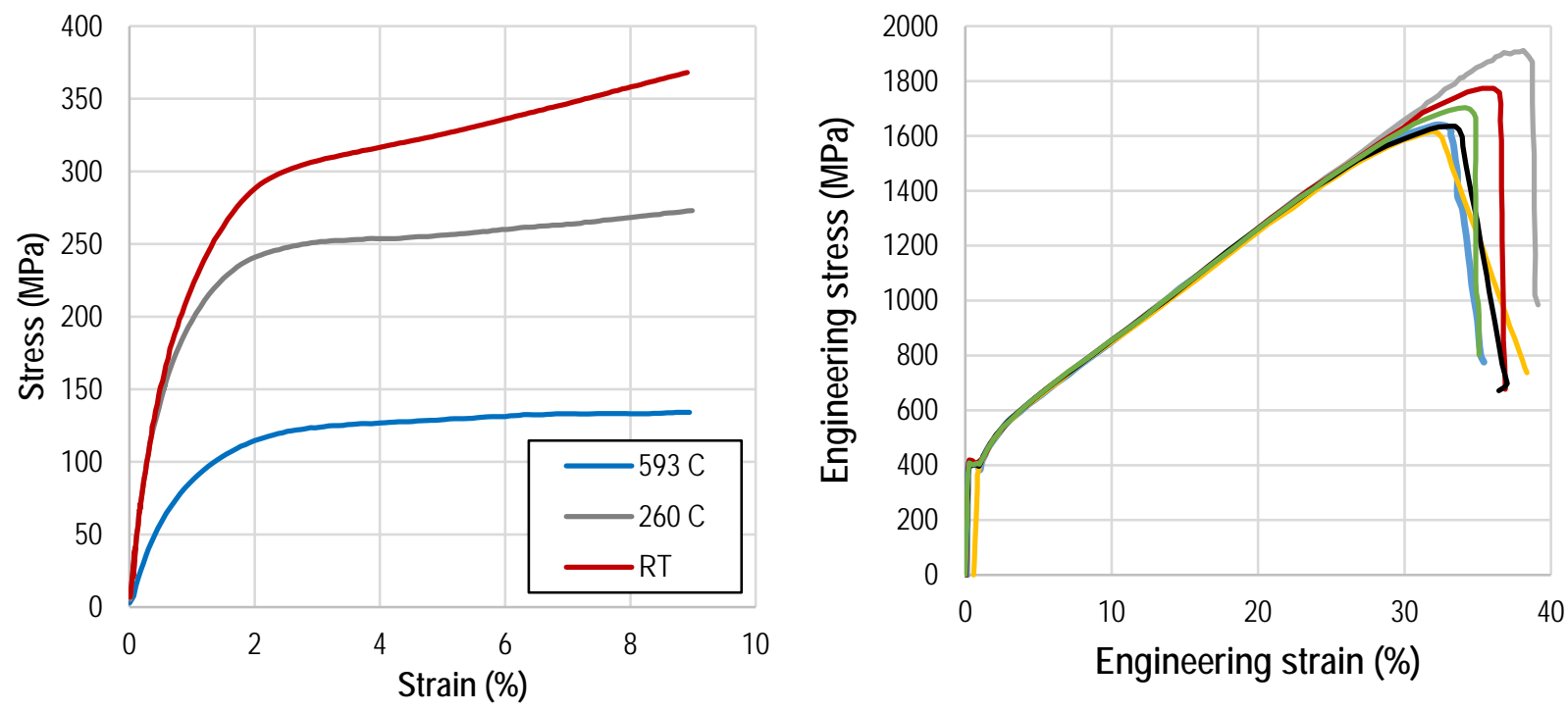

Figure 3.1. Stress-strain curves from tensile-strength test for S-200-F grade (left) and compressive-strength test at room temperature for S-200-FH grade (right)

Figure 3.2 (left) shows the decrease of Young's modulus with increasing temperature for S-200-F grade (both plots digitized from [20]). The initial drop is accelerated at temperatures above $400^{\circ} \mathrm{C}$. In the range of interest $\left(60\right.$ to $100^{\circ} \mathrm{C}$ ), the decrease in Young's modulus is minimal. Also, a lower Young's modulus results in lower stresses. Young's modulus, which is a measure of the interatomic bond strength, has been determined to be insensitive to the method of fabrication [46]. Thus, very similar behavior is expected for S-200-FH grade. In this analysis, Young's modulus was conservatively assumed to be temperature independent. Figure 3.2 (right) presents the plot of elongation at break 
as a function of temperature for S-200-F grade. The ductility increases with the temperature up to about $400^{\circ} \mathrm{C}$ and then decreases. This property is not directly used in the analysis, but it indicates that in the expected range of temperatures for MURR beryllium, the ductility will be higher than that reported at RT.
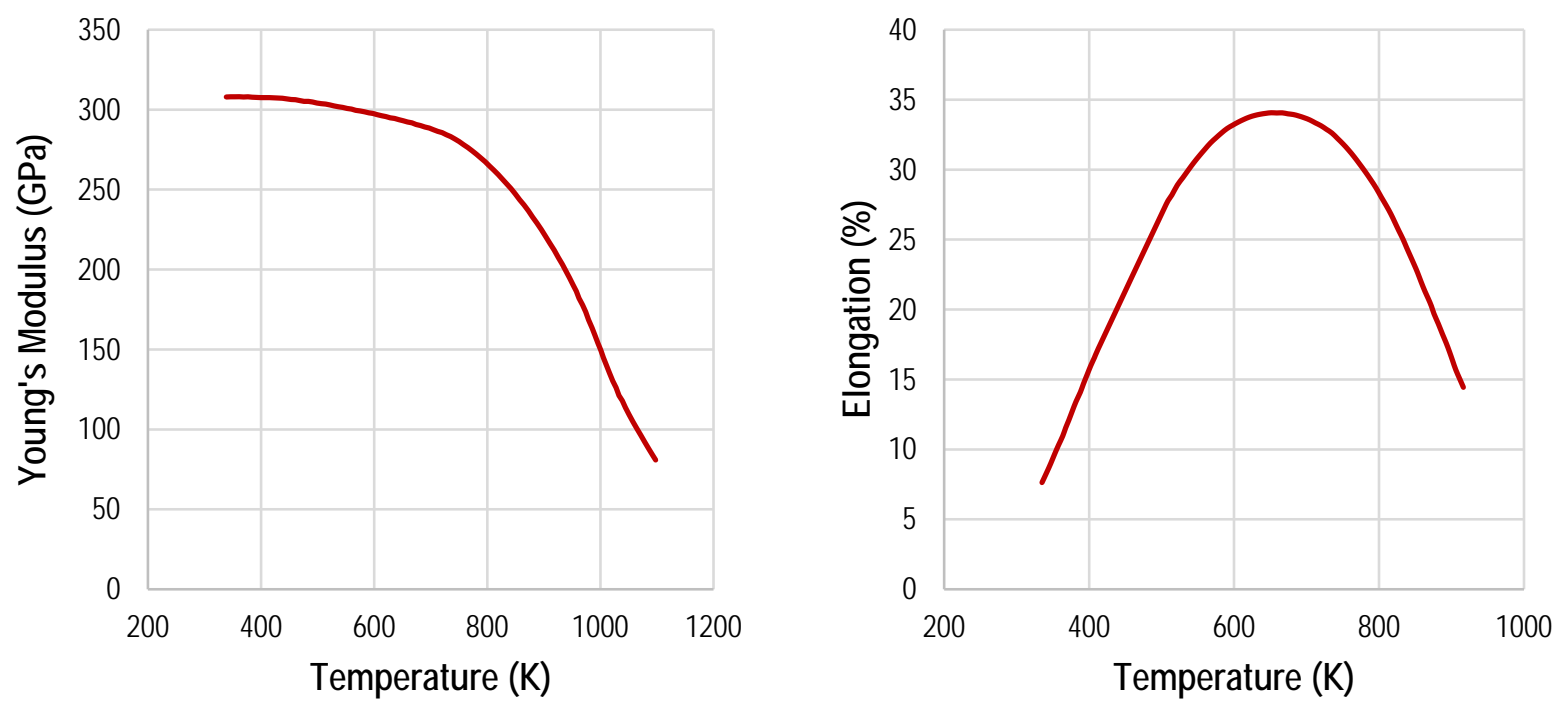

Figure 3.2. Temperature dependency of Young's modulus and elongation at failure for S-200F grade

Figure 3.3 presents the degradation of yield strength and UTS as a function of temperature for S-200FH and S-200-F grades (digitized from [47]). The trends are similar for both grades, but the strength at RT is different. UTS for S-200-FH at RT is $452.3 \mathrm{MPa}$ (consistent with the data in Table 3.1). The degradation due to temperature increase is linear. At the outer surface of the beryllium, where the temperature reaches 60 to $65^{\circ} \mathrm{C}$, the UTS can be assumed to be approximately $430 \mathrm{MPa}$ in the fresh, unirradiated state.
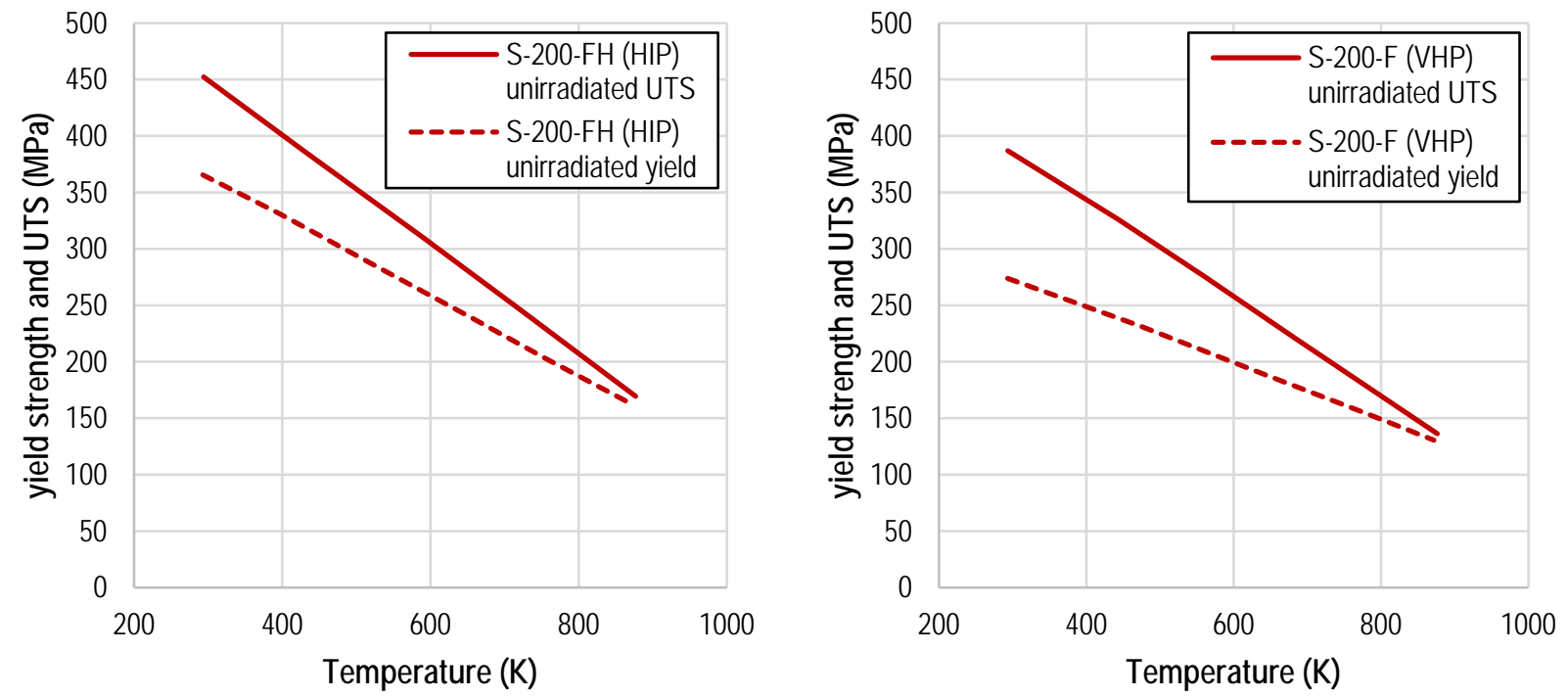

Figure 3.3. Dependency of yield strength and UTS on temperature for S-200-FH (left) and S200-F (right) beryllium 
In the fresh state, S-200-F and S-200-FH have the same coefficient of thermal expansion (CTE) reported by Materion (see Table 3.1) and are not expected to vary drastically across the temperatures of interest. Figure 3.4 presents the CTE of various grades of beryllium as a function of temperature (data sets digitized from [25]). On the left, properties of S-200-F grade are presented, while on the right, the CTEs of two (unspecified) grades produced by Materion with different $\mathrm{BeO}$ contents are presented. The CTE increases with increasing temperature. This behavior contributes to more expansion and larger stresses due to the heat. On the outer surface, at the mid-height of the MURR beryllium reflector, where a temperature of about $65^{\circ} \mathrm{C}$ is predicted, the CTE may reach about $12.5^{*} 10^{-6} / \mathrm{K}$.
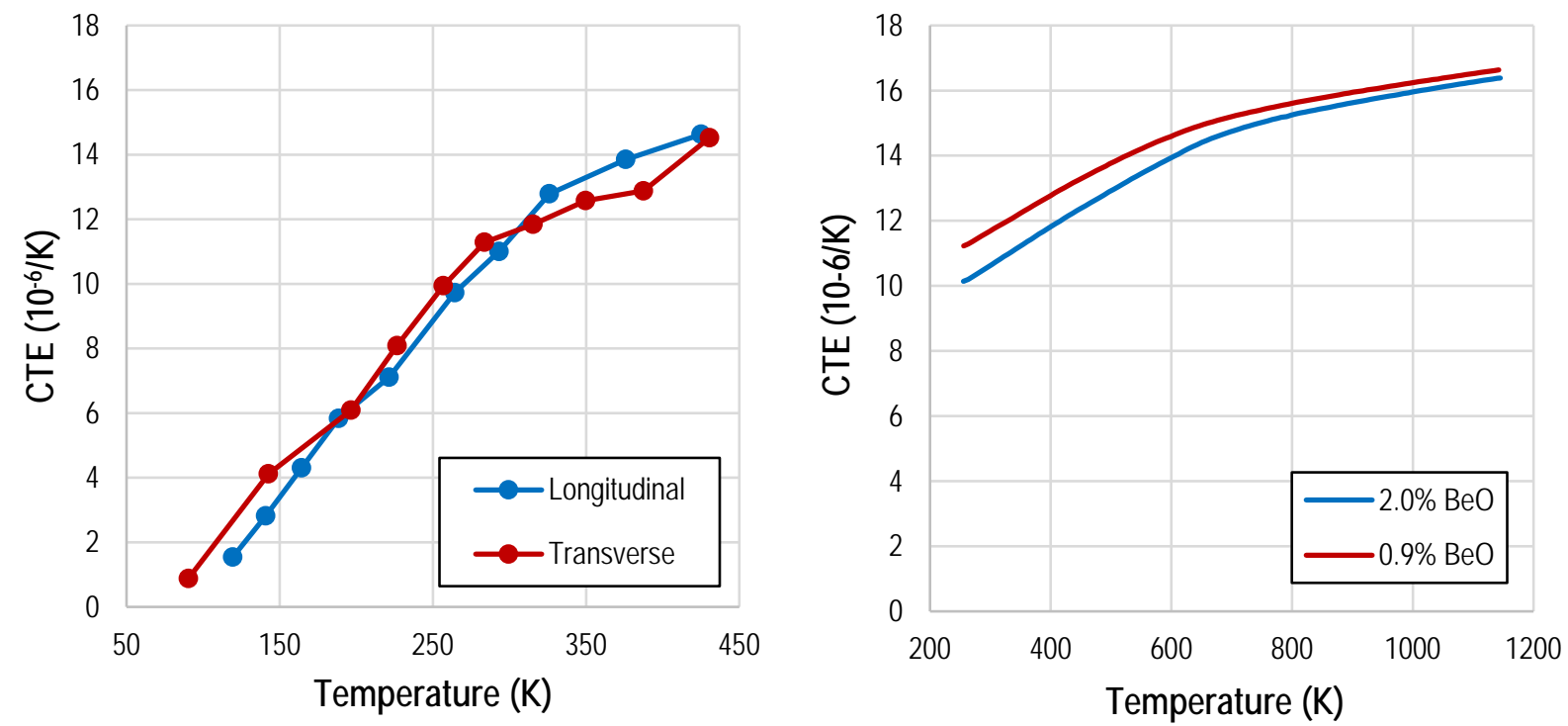

Figure 3.4. Coefficient of thermal expansion of S-200-F grade (left) and two other unspecified grades produced by Materion with different BeO contents (right)

Figure 3.5 and Figure 3.6 show thermal conductivity as a function of temperature for various grades of beryllium produced by Materion and a Russian vendor, respectively (digitized from [48], [25], and [49]). Similar trends are observed for all grades. Specifications for S-200-F and S-200-FH (Table 3.1) list a thermal conductivity of $216 \mathrm{~W} / \mathrm{m}-\mathrm{K}$ for both of these grades at RT. However, an extensive report from Materion [25] lists a measured value of $204 \mathrm{~W} / \mathrm{m}-\mathrm{K}$ for S-200-F grade. Lower thermal conductivity leads to higher thermal stresses. Although the true dependency of thermal conductivity on the temperature for S-200-FH could not be found in the literature, a decrease of about $10 \%$ from the value measured at RT to the value at $65^{\circ} \mathrm{C}(338 \mathrm{~K})$ predicted on the outer side of the reflector is to be expected. 

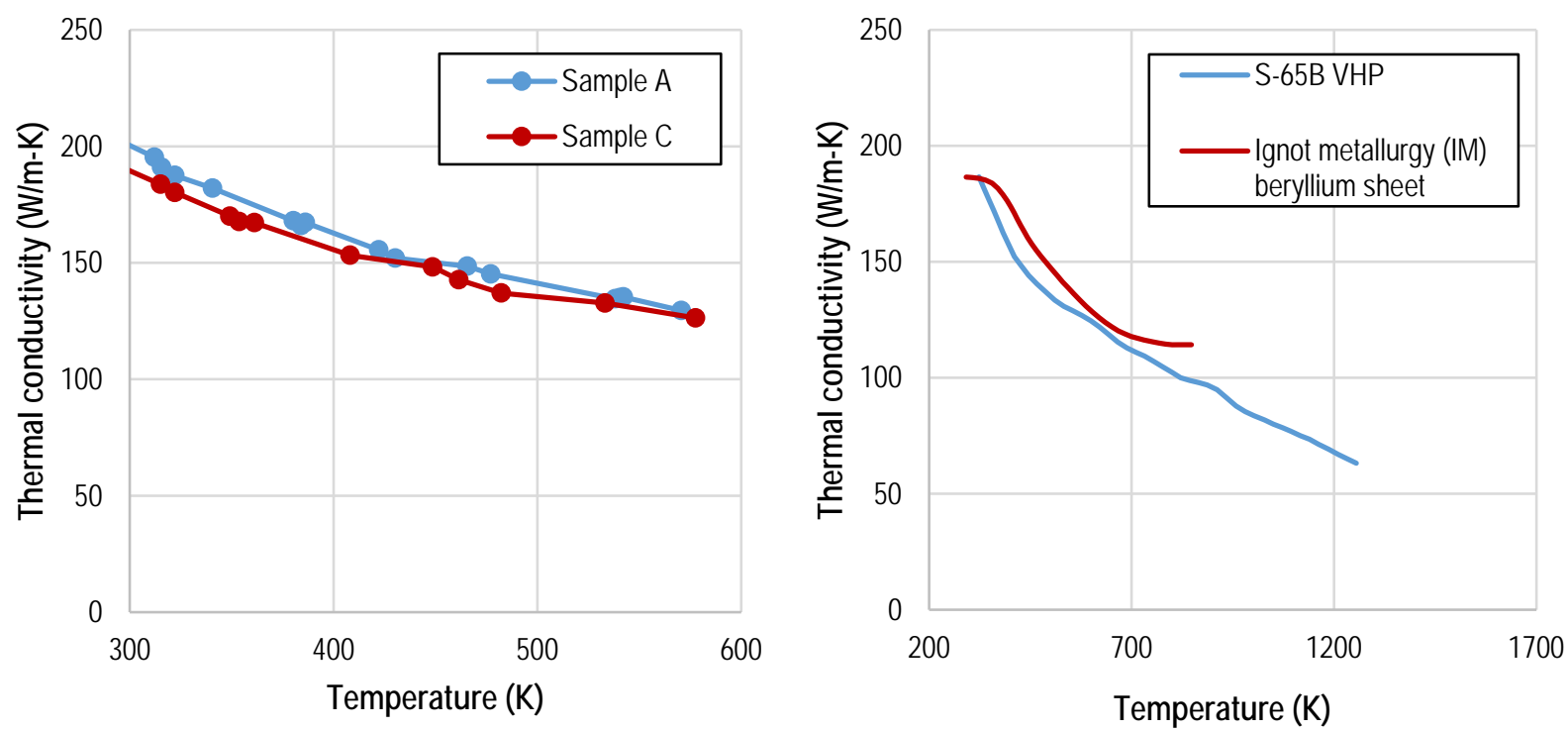

Figure 3.5. Thermal conductivity of hot-pressed beryllium produced by Brush Wellman (Materion's predecessor) in the 1970s (left) and produced more recently by Materion (right)

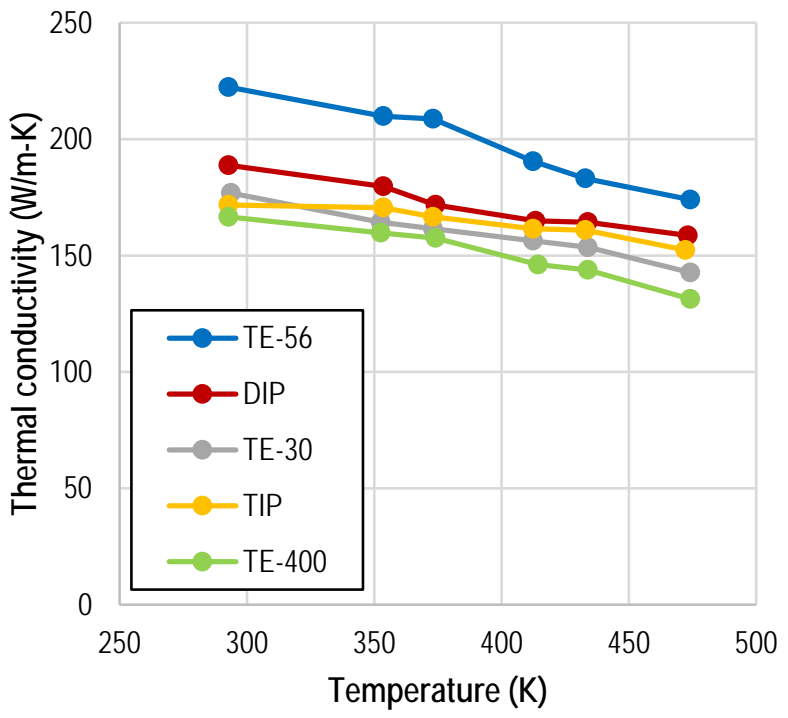

Figure 3.6.Thermal conductivity of several beryllium grades produced in Russia

Figure 3.7 shows the dependency of fracture toughness on the temperature for fresh (unirradiated) beryllium. The plot on the left was created from the data obtained for four grades produced by Materion (S-200-F, S-200-FH, S-65, and S-65-H) and tested at BR2 [42]. The data presented in the plot on the right were obtained for an older grade, S-200-E [25]. Additional data on fracture toughness of fresh S-65 and S-200-E grades can be found in [50]. Temperature dependency of fracture toughness of CIP-HIP grade beryllium is presented in Figure 3.8 [51].

The fracture toughness of beryllium increases with increasing temperature. No obvious effect of the material grade on the fracture toughness was found in this study. While it is possible that for highly irradiated beryllium, the grade no longer influences the strength, in the fresh state, there are substantial differences in the yield strength, UTS, and elongation at break for various grades of beryllium. 

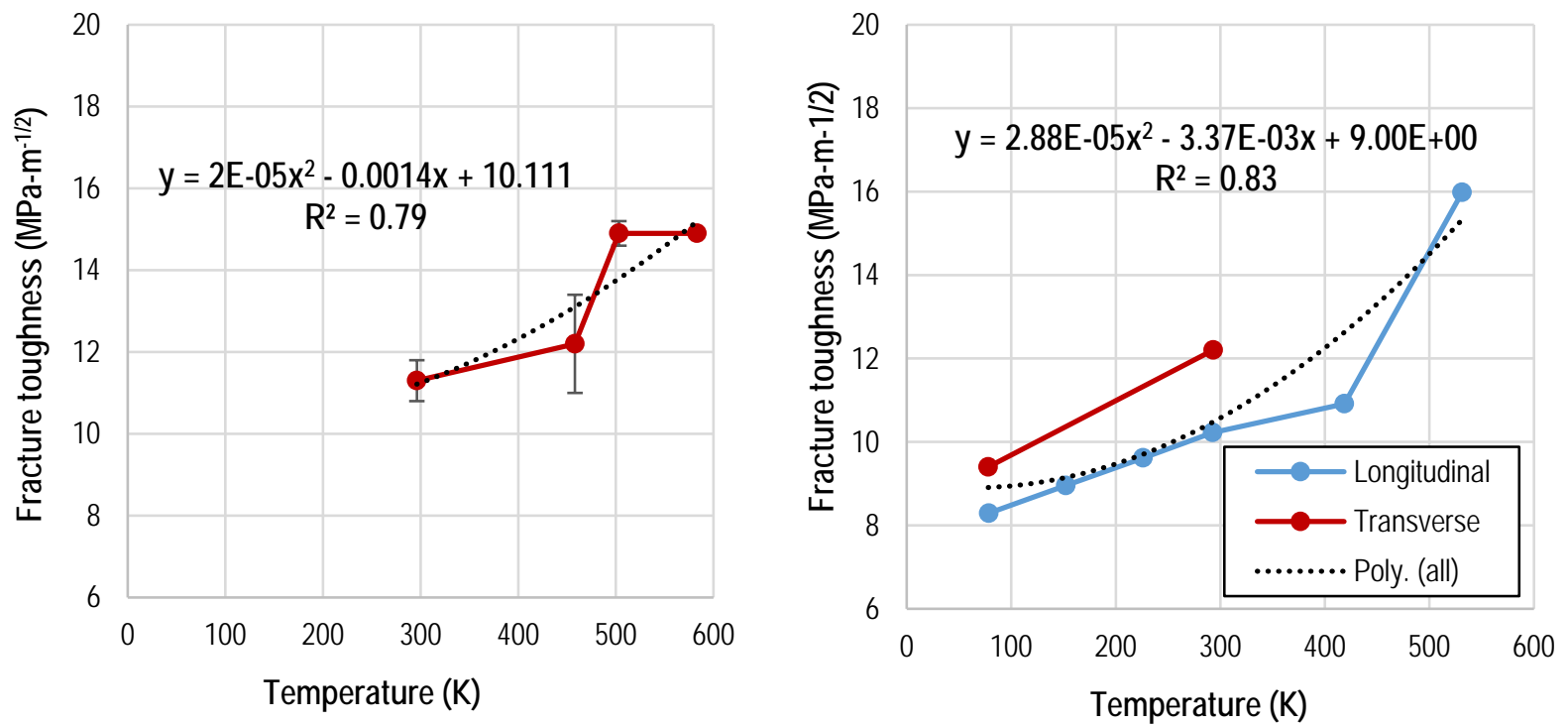

Figure 3.7. Dependency of fracture toughness of beryllium on temperature: collective data for S-200-F, S-200-FH, S-65, S-65-H grades (left); data for S-200-E (right)

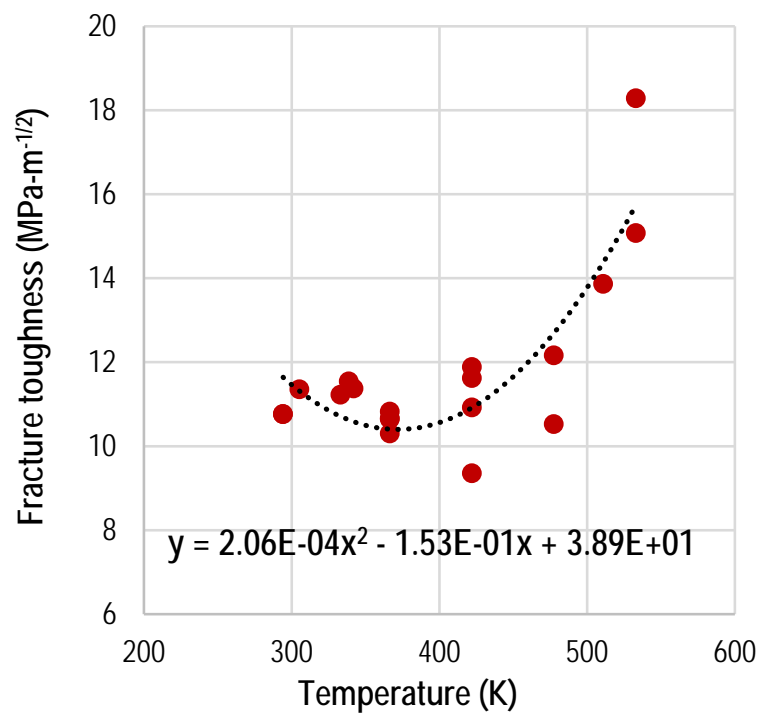

Figure 3.8. Dependency of fracture toughness of beryllium on temperature for CIP-HIP grade

Based on the data presented in this section, the material properties listed in Table 3.2 have been assumed for the finite element model at the fresh, unirradiated state (at $65^{\circ} \mathrm{C}$ ).

Table 3.2. Material properties assumed for the FE model at fresh, unirradiated state.

\begin{tabular}{|l|c|}
\hline Young's modulus (GPa) & 307.7 \\
\hline Poisson's ratio (-) & 0.04 \\
\hline CTE (ppm/K) & 12.5 \\
\hline Thermal conductivity (W/m-K) & 204 \\
\hline Heat capacity (J/kg-K) & 1925 \\
\hline density $\left(\mathbf{k g} / \mathbf{m}^{\mathbf{3}}\right.$ ) & 1850 \\
\hline
\end{tabular}




\subsection{Effect of Irradiation on Material Properties of Beryllium}

Data available in the open literature on specific irradiation-induced degradation of the thermal or mechanical properties of beryllium from reactor sites are limited. The data pertain to various beryllium grades, and the rate of degradation is most often given as a function of fast fluence (neutrons with energy $>0.1 \mathrm{MeV}$ in most references, while some references consider energies $>1.0$ $\mathrm{MeV}$ ). Since the neutron energy spectra differ from one reactor to another, the same cumulative fastneutron fluence level may have, to some degree, a different effect on material properties of beryllium samples irradiated at different reactor sites with similar neutron fluence. Consequently, the data obtained at one reactor site must be used with caution to evaluate changes in beryllium properties of samples irradiated at another location, particularly for non-energy-threshold effects. In this work, we attempt to convert available irradiated-beryllium property change data into data in terms of dpa and He- 4 content. Irradiation displacement damage, measured in dpa, is a quantity that describes the effects of irradiation on material structure. The damage is much more independent of the irradiation facility than is the fluence, and thus it should be the primary basis of the description of the property changes [7], although this has not uniformly been the case in past work. However, the methodology used for determining the damage (dpa) should also be reported.

Other secondary factors, like transmutation rates or type of irradiation (pulsed or continuous), may play a significant role in property degradation as well. However, in the case of the MURR beryllium reflector, as well as in the case of other research reactors, the production rate of transmutation gases is very slow and these other factors are irrelevant.

As concluded in several studies, including [52] and [53], four irradiation temperature regimes of interest for beryllium are usually identified:

1. In the low-temperature regime $\left(\lesssim 20^{\circ} \mathrm{C}\right)$, immobile point defects can be created and coalescence of these defects is very unlikely.

2. In the intermediate-temperature regime between $20^{\circ} \mathrm{C}$ and $300^{\circ} \mathrm{C}$, defects become mobile, but the produced gases are still immobile and stay at the locations where they were produced in the crystals. Swelling in this case is referred to as cold swelling.

3. At high temperatures between $300^{\circ} \mathrm{C}$ and $600^{\circ} \mathrm{C}$ gas atoms become mobile.

4. At very high temperatures $>600^{\circ} \mathrm{C}$, gas-driven swelling becomes dominant.

For low- and intermediate-temperature irradiations (regimes 1 and 2), damage in terms of dpa should be used as the measure of property degradation, while for higher temperatures $\left(>300^{\circ} \mathrm{C}\right)$, mobility of He-4 atoms also needs to be taken into account [54].

It is important to note that the rate of degradation of thermo-mechanical properties for irradiated beryllium strongly depends not only on irradiation temperature, but also on post-irradiation annealing and testing temperatures. Collected data must correspond to the conditions experienced by the MURR beryllium reflector during operation. The analysis presented here indicates that the maximum temperature in the beryllium reflector is below $100^{\circ} \mathrm{C}$ (see Section 4.6). Thus, the data collected for the empirical material models presented below correspond only to samples irradiated, as well as tested, at around $100^{\circ} \mathrm{C}$, without annealing at elevated temperatures preceding the testing.

The MURR beryllium reflector material (S-200-FH) is fabricated using a HIP process; ATR and BR2 are currently using S-200-F beryllium manufactured by VHP. These three reactors have previously contracted with Materion (formerly Brush Wellman) for beryllium reflector production. The high-flux SM3 reactor located in Dimitrovgrad, Russia, uses TE-56 hot extruded beryllium for its reflector. The 
TE-56 grade differs in composition (purity of beryllium), grain size, and isotropy from the S-200-FH grade used by MURR. S-200-FH, as fresh material, is the most isotropic and strongest among these. Unfortunately, very few of the properties of interest for this study are available for S-200-FH grade under irradiation. In the absence of available data, the authors have made an assumption that the property degradation reported for the grades developed earlier (prior to introduction of S-200-F and S-200-FH) and used at other reactors will be a sufficient substitute for S-200-FH grade beryllium data. The strength limits of these earlier grades in the fresh state are lower than the strength limits of S200-FH grade. Thus, the authors believe that these assumptions lead to conservative underestimations of the MURR beryllium reflector life. However, until proper strength testing of highly irradiated S-200-FH is performed, this assumption is not certain. As will be shown later in this chapter, higher fresh-state strength for a specific grade doesn't guarantee a higher strength in the irradiated state as compared to other grades with lower fresh-state strength.

Of highest interest for beryllium lifetime analysis in this work are the properties and behavioral models covered in subsequent sections:

1. Thermal conductivity - used for thermal analysis and prediction of temperature-induced stresses

2. Swelling rate due to transmutation products - used for prediction of internal stresses

3. Tensile yield strength - used for failure analysis

4. Compressive yield strength - used for failure analysis

5. Fracture toughness - used for failure analysis

Beryllium reflectors in research and test reactors such as MURR, ATR, BR2, SM and others are usually experiencing irradiation temperatures below $100^{\circ} \mathrm{C}$ (i.e., temperatures in the low- and intermediatetemperature regimes defined above). On the basis of an open-literature survey, there is a significant drop in thermal conductivity from $220 \mathrm{~W} / \mathrm{m}-\mathrm{K}$ (fresh beryllium) to about $50 \mathrm{~W} / \mathrm{m}-\mathrm{K}$ after irradiation at low temperatures and fast-neutron fluence $(\mathrm{E}>0.1 \mathrm{MeV})$ of about $2 * 10^{22} \mathrm{n} / \mathrm{cm}^{2}$ and beyond [13], [55]. Concurrently, irradiation hardening and embrittlement in irradiated beryllium material can initially result in a significant gain in the UCS of the material, as ATR data indicate [8]. Up to a 50\%

gain in UTS is also noted. Further irradiation leads to a complete loss of ductility and decrease of the strength below the levels present in fresh beryllium. The irradiated material becomes very brittle and brittle fracture is the dominant mode of failure in this case. Since neutron exposure, and thus the damage level, is not constant throughout the beryllium reflector in MURR, multiple modes of failure (ductile, brittle) are possible.

Thermal-conductivity degradation data at low temperature and high neutron fluence (up to $12 * 10^{22}$ $\mathrm{n} / \mathrm{cm}^{2}, \mathrm{E}>0.1 \mathrm{MeV}$ ) are available only from the SM reactor in Russia. Swelling data are most abundant, and come primarily from SCK, SM, and ATR, although other sources also provide some data. The most complete data on tensile and compressive strength of beryllium come from tests performed at ATR in the late 1970s and early 1980s [8]. Fracture-toughness data for irradiated beryllium come from the ATR, SCK, and SM reactors, although the data on beryllium fracture toughness from SCK and ATR are limited.

\subsubsection{Scaling of Data from Various Reactor Sites}

The material properties data for various grades of beryllium under irradiation needed for the current work have been reported in the literature. Such data are not usually presented as a function of all three irradiation parameters of fast fluence, He-4 content, and damage (dpa), but are rather presented as a function of just one or two of these parameters. Frequently, when fluence is given as a 
measure of irradiation level, two neutron energy ranges are considered, $\mathrm{E}>0.1 \mathrm{MeV}$ and $\mathrm{E}>1.0 \mathrm{MeV}$. Data on fluence of neutrons in these energy ranges, He-4 content, and damage have been collected for several reactor facilities at which beryllium has been tested, and allow for calculating the empirical relationships between these irradiation parameters.

Most of the data on behavior and properties of beryllium irradiated at low temperatures (below $100^{\circ} \mathrm{C}$ ) come from three reactors: SM (swelling, thermal conductivity, tensile strength, compressive strength), BR2 (swelling, tensile strength, fracture toughness), and ATR (swelling, tensile strength, compressive strength, fracture toughness). For that reason, conversion factors for these three reactors are of most importance.

Table 3.3 lists the scaling factors that have been calculated between fast fluence, He- 4 content, and damage for irradiated beryllium at these reactor sites. While these relationships are not truly linear, a linear relationship is usually a close approximation for the irradiation conditions of interest. Figure 3.9 shows relationships between fluence and He- 4 content and fluence and damage, respectively, for HEU and LEU cores in MURR, based on the MCNP5 calculations completed in this work. The conversion factors for MURR in Table 3.3 were calculated as an average of the values for the HEU and LEU cores. The set of data for SM, BR2, and HFIR used to build this table is included in Appendix E.

Table 3.3. Relationships between fluence, He-4 content, and dpa for irradiated beryllium at various reactor sites

\begin{tabular}{|c|c|c|c|c|c|c|c|}
\hline Reactor site & SM & BR2 & ATR & HFIR & HFBR & BOR & MURR \\
\hline $\begin{array}{l}\text { Ratio of fluence } \mathrm{E}>0.1 \mathrm{MeV} \\
\text { and fluence } \mathrm{E}>1.0 \mathrm{MeV}\end{array}$ & $2.035^{*}$ & $1.991^{*}$ & -- & 1.876 & $2.381^{*}$ & $2.00^{*}$ & 2.39 \\
\hline $\begin{array}{l}\text { Ratio of fluence } \mathrm{E}>0.1 \mathrm{MeV} \\
\left({ }^{*} 10^{22} \mathrm{n} / \mathrm{cm}^{2}\right) \text { and } \mathrm{He} \\
\text { content }(\mathrm{appm})\end{array}$ & 1677 & 2530 & -- & 2081 & $5000^{*}$ & $612.5^{*}$ & 1243 \\
\hline $\begin{array}{l}\text { Ratio of fluence } \mathrm{E}>0.1 \mathrm{MeV} \\
\left({ }^{*} 10^{22} \mathrm{n} / \mathrm{cm}^{2}\right) \text { and damage } \\
(\mathrm{dpa})\end{array}$ & 5.179 & $6.129 *$ & -- & 8.192 & $6.800^{*}$ & $5.875^{*}$ & 5.619 \\
\hline $\begin{array}{l}\text { Ratio of fluence } \mathrm{E}>1.0 \mathrm{MeV} \\
\left({ }^{*} 10^{22} \mathrm{n} / \mathrm{cm}^{2}\right) \text { and } \mathrm{He} \\
\text { content }(\mathrm{appm})\end{array}$ & $3413^{*}$ & 4494 & 4700 & 3920 & $11905^{*}$ & $1225^{*}$ & 2985 \\
\hline $\begin{array}{l}\text { Ratio of fluence } \mathrm{E}>1.0 \mathrm{MeV} \\
\left(* 10^{22} \mathrm{n} / \mathrm{cm}^{2}\right) \text { and damage } \\
(\mathrm{dpa})\end{array}$ & $10.54^{*}$ & 12.20 & $3.50^{*}$ & 15.43 & $16.19^{*}$ & $11.62^{*}$ & 13.44 \\
\hline $\begin{array}{l}\text { Number of references used } \\
\text { to build the data set }\end{array}$ & $\begin{array}{c}10 \\
\text { [56], [57], } \\
\text { [58], [59], } \\
\text { [12], [60], } \\
\text { [14], [13], } \\
\text { [49], [55] }\end{array}$ & $\begin{array}{c}7 \\
{[56],[61],} \\
{[42],[15],} \\
{[62],[63],} \\
{[14]}\end{array}$ & $\begin{array}{c}3 \\
{[64],[65]} \\
{[66]}\end{array}$ & $\begin{array}{c}1 \\
{[10]}\end{array}$ & $\begin{array}{c}1 \\
{[10]}\end{array}$ & $\stackrel{2}{2}[56],[57]$ & -- \\
\hline
\end{tabular}

Note: numbers marked with * symbol are based on a single data point

It can be noted that the ratio of the neutron fluence for $\mathrm{E}>0.1 \mathrm{MeV}$ and $\mathrm{E}>1.0 \mathrm{MeV}$ is similar for all reactors and is in the range between 1.88 and 2.39. The ratio of the neutron fluence for $\mathrm{E}>1.0 \mathrm{MeV}$ $\left(* 10^{22} \mathrm{n} / \mathrm{cm}^{2}\right)$ and He content (appm) is between $\sim 3,000$ and 4,700 for all but two reactors. For those reactors, HFBR and BOR, only one data point was available for each.

The ratio of the neutron fluence for $\mathrm{E}>1.0 \mathrm{MeV}\left(* 10^{22} \mathrm{n} / \mathrm{cm}^{2}\right)$ and damage (dpa) is between 10.54 and 16.19 for all reactors but ATR. Only one data point [66] for the calculated ratio of fluence for E > $1.0 \mathrm{MeV}$ and dpa is available for ATR; that ratio is 3.5, a vastly different value from those of the other 
reactors for which data are available. No data for calculating the ratio of neutrons with $\mathrm{E}>0.1 \mathrm{MeV}$ to neutrons with $\mathrm{E}>1.0 \mathrm{MeV}$ are available for the ATR reflector. For the ATR, although three data points were available for ratios between fluence for $\mathrm{E}>1.0 \mathrm{MeV}$ and $\mathrm{He}-4$ content, only one of them, 4,700 [66], [65], accounted for total He-4 production (footnote of Table 2 in [65]); that data point was used in Table 3.3.
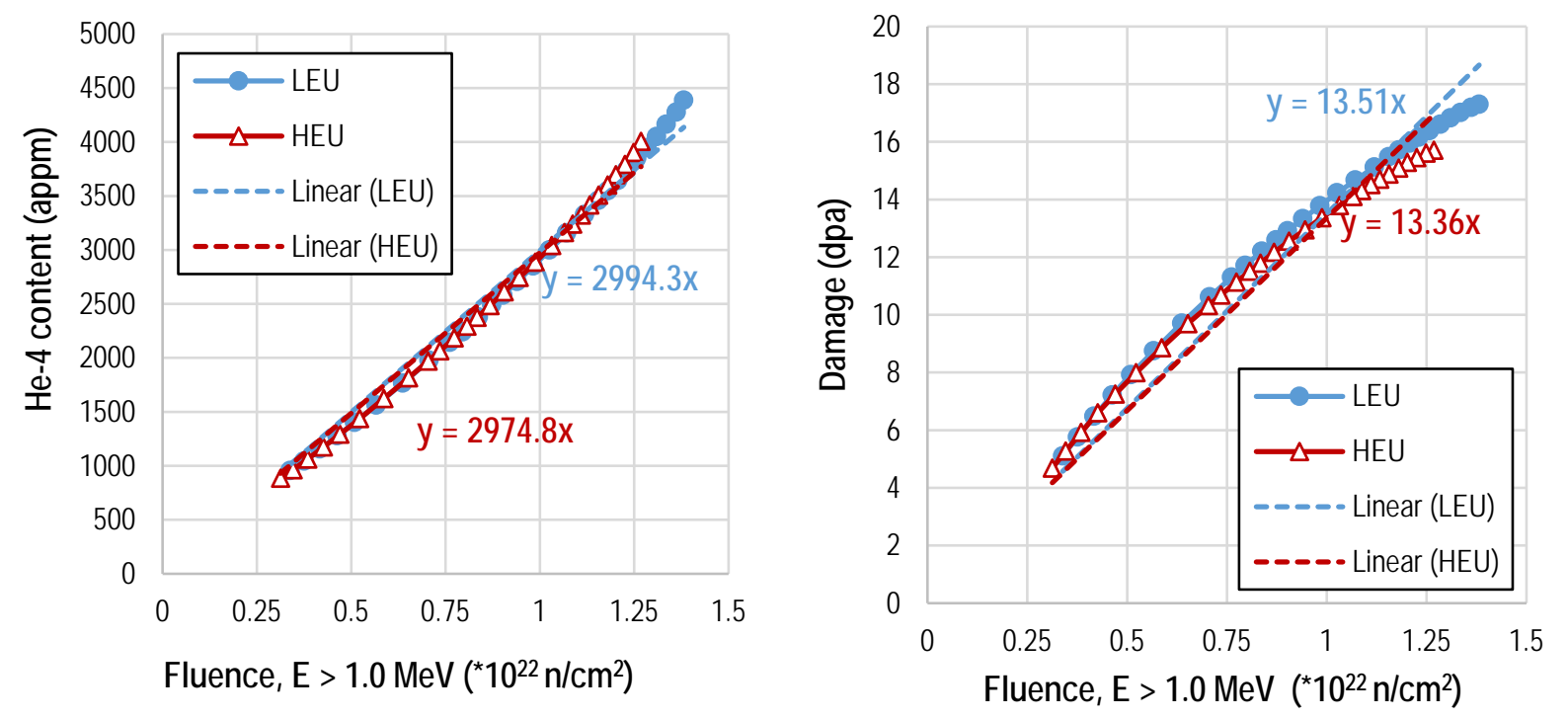

Figure 3.9. Relationship between fluence $(E>1.0 \mathrm{MeV}$ ) and He-4 content (left) and fluence (E $>1.0 \mathrm{MeV}$ ) and damage (right) in MCNP5 calculations for MURR beryllium reflector

\subsubsection{Thermal Conductivity}

This section presents the data on thermal conductivity of beryllium irradiated at low temperatures $\left(\sim 70^{\circ} \mathrm{C}\right)$ similar to the temperatures experienced by the MURR beryllium reflector. Data on thermal conductivity of irradiated beryllium are scarce in the open literature, and the data set presented here comes exclusively from the experiments conducted at the SM reactor in Russia ( [13], [49], [55], [56], [60]). The data were collected for several grades of beryllium produced in Russia that have various chemical compositions and are made with various powder compaction techniques, resulting in a broad range of thermomechanical properties [49]. The thermal conductivity of TE-30, TE-400, TIP, and DIP grades in the fresh (unirradiated) state is lower than the thermal conductivity of S-200-F and S-200-FH grades (see Figure 3.6). Only TE-56 grade has thermal conductivity in the unirradiated state similar to the conductivity of S-200-F and S-200-FH grades, i.e., $222 \mathrm{~W} / \mathrm{m}-\mathrm{K}$ versus $216 \mathrm{~W} / \mathrm{m}-\mathrm{K}$ respectively. The most extensive set of data for thermal conductivity of beryllium in the irradiated state was available for the TE-56 grade.

Thermal conductivity decreases monotonically with irradiation expressed as either fast neutron fluence or damage, but the decrease is not linear. As shown in Figure 3.10, a significant reduction of the thermal conductivity occurs for fast fluence $(\mathrm{E}>0.1 \mathrm{MeV})$ up to $2.0^{*} 10^{22} \mathrm{n} / \mathrm{cm}^{2}$. Beyond these values, the rate of further degradation slows down and levels off at around $50 \mathrm{~W} / \mathrm{m}-\mathrm{K}$. The collected data indicate that the thermal-conductivity degradation is relatively independent of the beryllium grade from which the irradiated samples were made. On the basis of that conclusion, it is assumed that data presented here can be used to construct thermal conductivity models for other beryllium grades, including S-200-FH and N-200-A, used in MURR reflectors. 


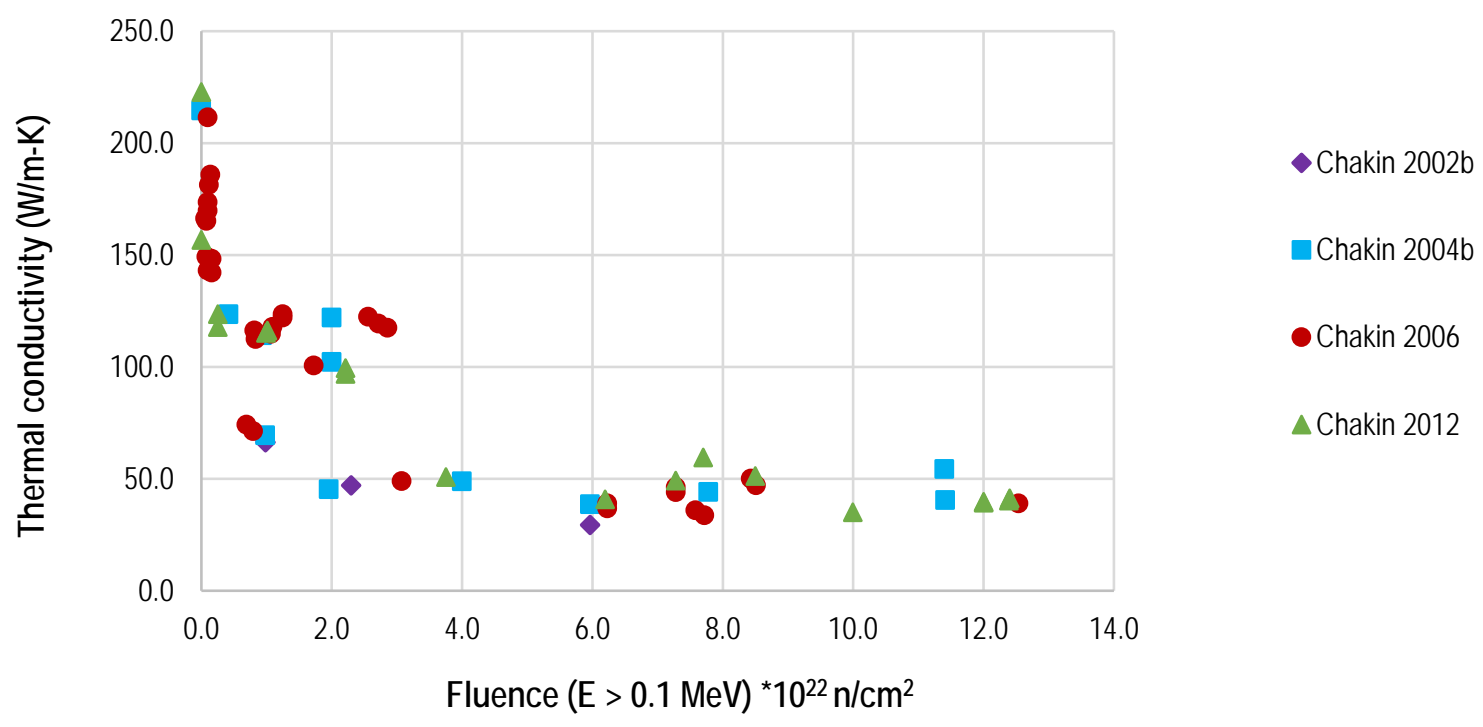

Figure 3.10. Thermal conductivity of irradiated beryllium as a function of fast neutron fluence $(\mathrm{E}>0.1 \mathrm{MeV})$

Figure 3.11 shows how the testing temperature influences the thermal conductivity of TE-56 grade in the irradiated state (data for several grades in the unirradiated state are shown in Figure 3.6) based on [60]. For irradiated samples, the testing temperature (in the tested range of up to $220^{\circ} \mathrm{C}$ ) appears to have no effect on thermal conductivity, whereas for unirradiated samples, increasing the testing temperature leads to a linear decrease of the thermal conductivity.

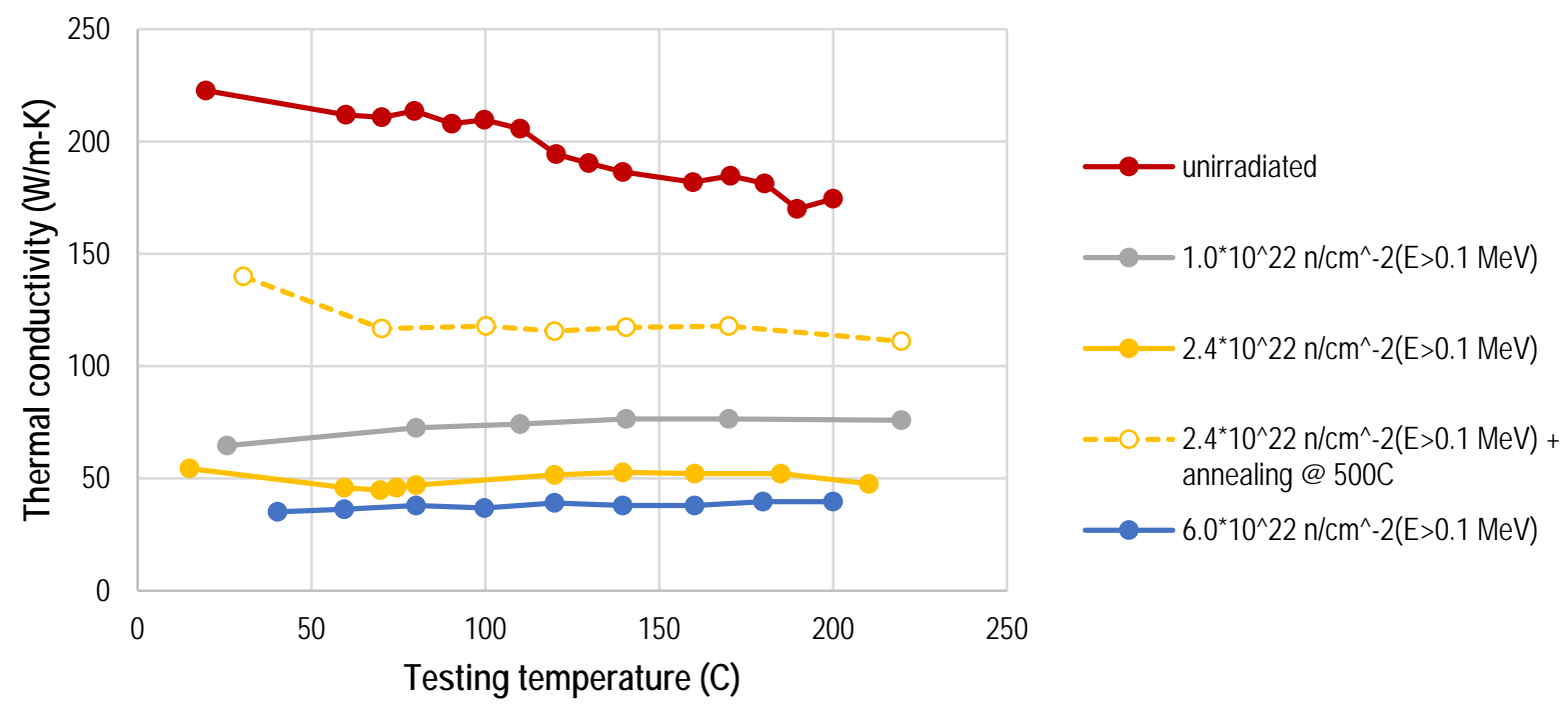

Figure 3.11. Dependence of the thermal conductivity of TE-56 grade beryllium on testing temperature

The thermal-conductivity data from Figure 3.10 are presented again in Figure 3.12 as a function of damage. To produce a thermal-conductivity model for subsequent FE analysis, a fourth-order polynomial-based fit to those data was performed. The starting point for the unirradiated state was set to $204 \mathrm{~W} / \mathrm{m}-\mathrm{K}$, which corresponds to the fresh-state thermal conductivity of S-200-FH at 
approximately $65^{\circ} \mathrm{C}$ (assuming temperature dependency based on plots in Figure 3.6). The polynomial fit was truncated at $20 \mathrm{dpa}$, beyond which point a constant thermal conductivity of 50 $\mathrm{W} / \mathrm{m}-\mathrm{K}$ was assumed. Additionally, a function representing the lower bound of the $95 \%$ confidence interval for the mean fit is presented. This fit was truncated at $13.35 \mathrm{dpa}$, beyond which point a constant value of $45 \mathrm{~W} / \mathrm{m}-\mathrm{K}$ was assumed. These fits are described by equations (3-1) and (3-2), respectively.

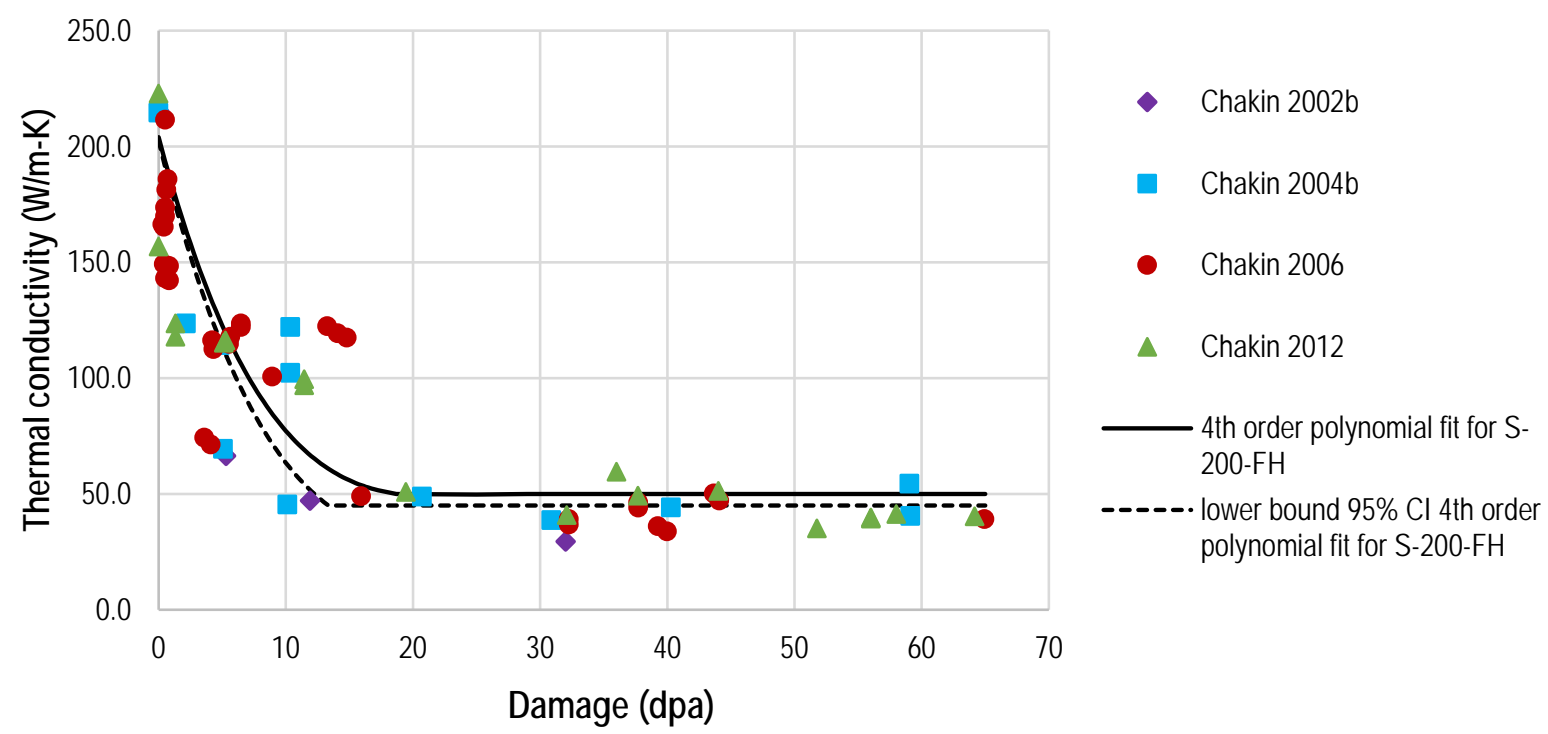

Figure 3.12. Thermal conductivity of irradiated beryllium as a function of damage in dpa, with a fit for S-200-FH grade beryllium

$$
\begin{aligned}
\text { for } d p a<25 & \\
\lambda_{\text {mean }}(d p a)= & 204-20.68 * \mathrm{dpa}+0.9795 * \mathrm{dpa}^{2}-0.01925 * \mathrm{dpa}^{3} \\
& +0.0001315 * \mathrm{dpa}^{4} \\
\text { for } d p a & >25, \quad \lambda_{\text {mean }}(d p a)=50 \\
\text { for } d p a & <13.35, \\
\lambda_{\text {lower fit }}(d p a)= & 202.5-22.43 * \mathrm{dpa}+1.037 * \mathrm{dpa}^{2}-0.01977 * \mathrm{dpa}^{3} \\
+ & 0.0001302 * \mathrm{dpa}^{4} \\
\text { for } d p a & >13.35, \lambda_{\text {lower fit }}(d p a)=45
\end{aligned}
$$

Similar fits have been performed to the same set of data but with a lower starting point for N-200-A grade. These fits are described by equations (3-3) and (3-4), and are shown in Figure 3.13. 


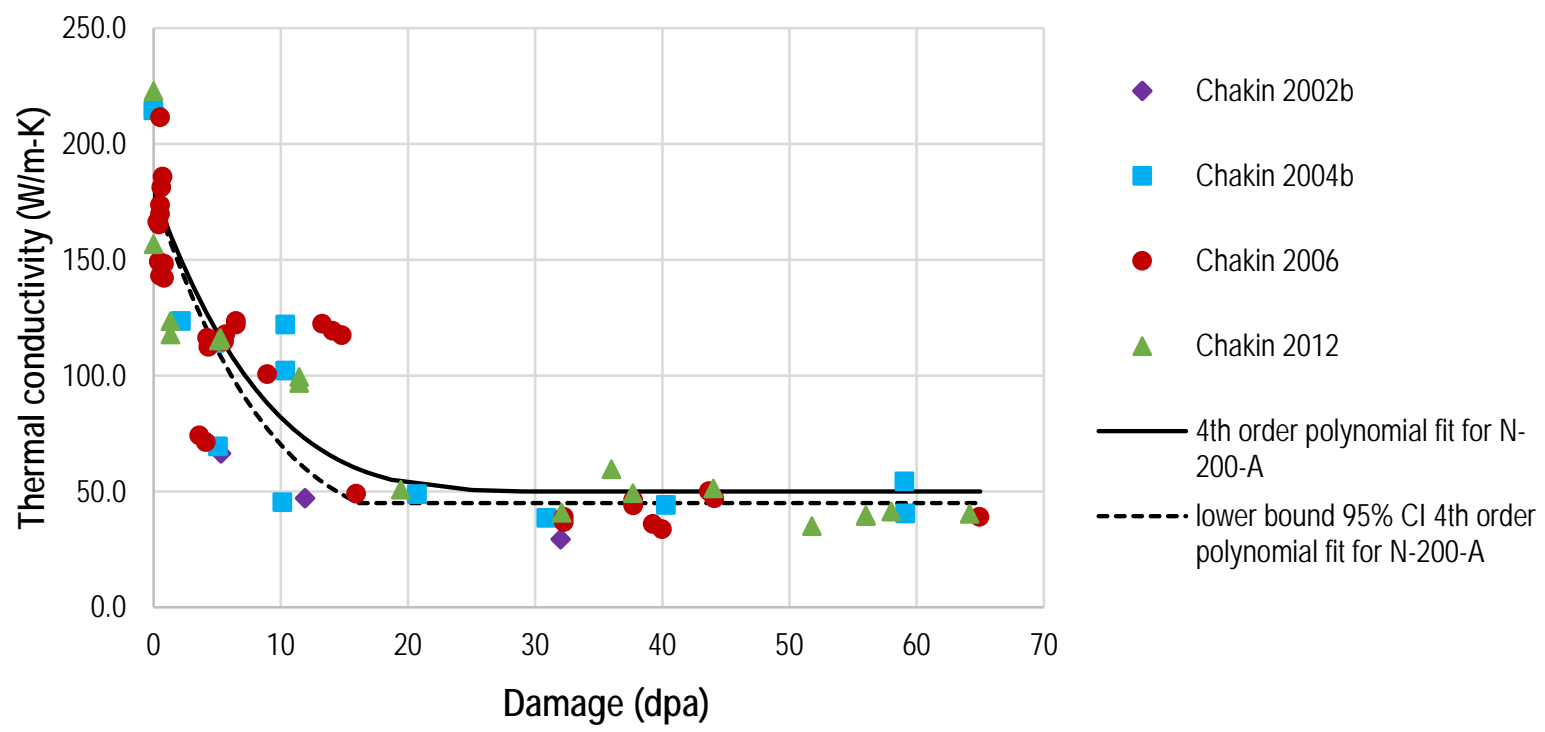

Figure 3.13. Thermal conductivity of irradiated beryllium as a function of damage in dpa, with a fit for $\mathrm{N}-200$-A grade beryllium

$$
\begin{aligned}
& \text { for dpa }<30, \\
\lambda_{\text {mean }}(d p a)= & 180-15.26 * \mathrm{dpa}+0.6614 * \mathrm{dpa}^{2}-0.01238 * \mathrm{dpa}^{3} \\
& +0.00008238 * \mathrm{dpa}^{4} \\
& \text { for dpa }>30, \quad \lambda_{\text {mean }}(\text { dpa })=50 \\
& \text { for dpa }<16.15, \\
\lambda_{\text {lower fit }}(d p a)= & 178.7-16.75 * \mathrm{dpa}+0.7104 * \mathrm{dpa}^{2}-0.01282 * \mathrm{dpa}^{3} \\
+ & 0.00008124 * \mathrm{dpa}^{4} \\
& \text { for dpa }>16.15, \quad \lambda_{\text {lower fit }}(\text { dpa })=45
\end{aligned}
$$

Thermal conductivity is distributed unevenly within the MURR reflector during its operation because of spatially and temporally varying neutron fluence. Figure 3.14 and Figure 3.15 present the distribution of thermal conductivity in the MURR beryllium reflector (S-200-FH grade) after 8 years of operation with HEU and LEU cores, respectively. The thermal-conductivity degradation fits presented above, as well as damage distributions presented in Section 2.3, were used to construct these figures. It can be seen that degradation in the beryllium reflector's thermal conductivity, after 8 years of operation, is slightly higher in the LEU core case than in the HEU core case because of the differences in radiation-induced damage. 

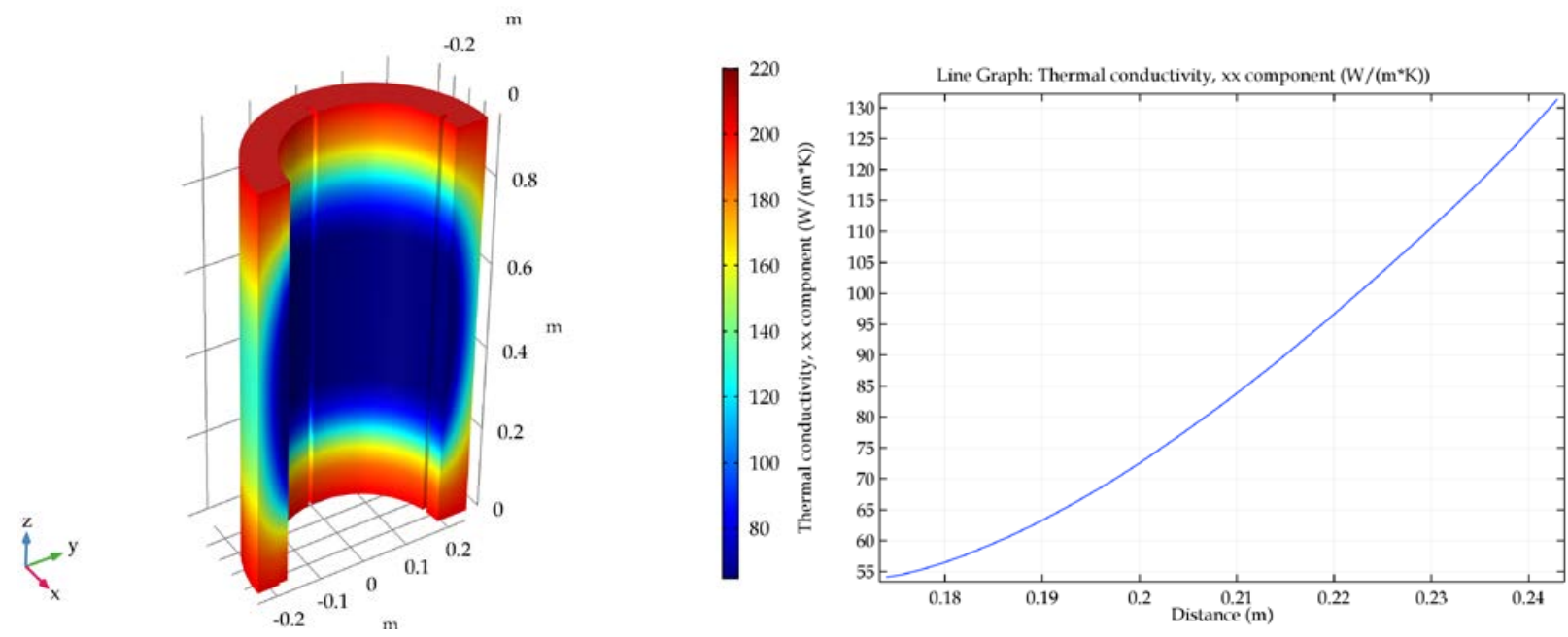

Figure 3.14. Thermal conductivity distribution in beryllium reflector irradiated for 8 years at $10 \mathrm{MW}$ with HEU core
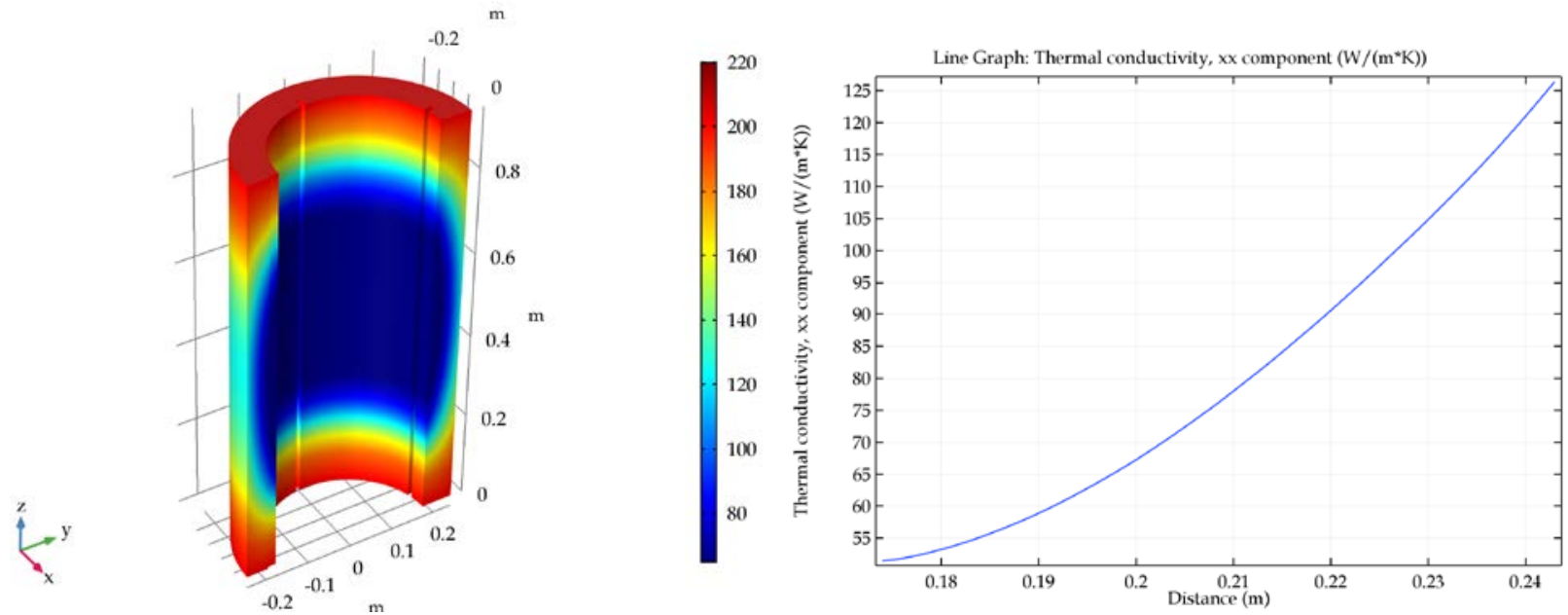

Figure 3.15. Thermal conductivity distribution in beryllium reflector irradiated for 8 years at 12 MW with LEU core

\subsubsection{Tensile Strength}

At RT, fresh, unirradiated beryllium behaves similarly to some other structural metals in both tension and compression. Raw data from tensile tests at various temperatures for S-200-FH grade [67] was digitized and is presented in Figure 3.16 (resolution loss is expected as a result of the digitization process). At RT, the linear elastic region is followed by a distinct yield point and a small Luders extension region (localized bands of plastic deformation). It is subsequently followed by a uniform work-hardening region up to the UTS at the fracture point with no prior necking. At $310^{\circ} \mathrm{C}$, upper and lower yield points can be identified, along with higher ductility and a significant necking after a small work-hardening region. At $605^{\circ} \mathrm{C}$, there is no indication of a yield point, the work hardening is very limited, and ultimate strength is reduced.

Figure 3.16 (right) shows stress-strain curves from RT tensile tests of another structural beryllium grade made by Materion, designated S-65-C [10]. Data are shown for an unirradiated sample as well as samples irradiated at HFIR and HFBR up to a fluence $(\mathrm{E}>0.1 \mathrm{MeV})$ of $0.2^{*} 10^{21} \mathrm{n} / \mathrm{cm}^{2}\left(\right.$ at $300^{\circ} \mathrm{C}$ ) and $0.5^{*} 10^{21} \mathrm{n} / \mathrm{cm}^{2}$ (at $205^{\circ} \mathrm{C}$ ), respectively. A similar grade of beryllium, S-65-B, irradiated at BR2 
up to a fluence $\left(\mathrm{E}>0.1 \mathrm{MeV}\right.$ ) of $0.65-0.85^{*} 10^{21} \mathrm{n} / \mathrm{cm}^{2}$ (at $185-310^{\circ} \mathrm{C}$ ) showed a total elongation of less than $0.1 \%$ [63]. Thus, with increasing irradiation dose, the ductility decreases while UTS increases. The Young's modulus appears not to be affected by the irradiation, and is assumed constant throughout this analysis.
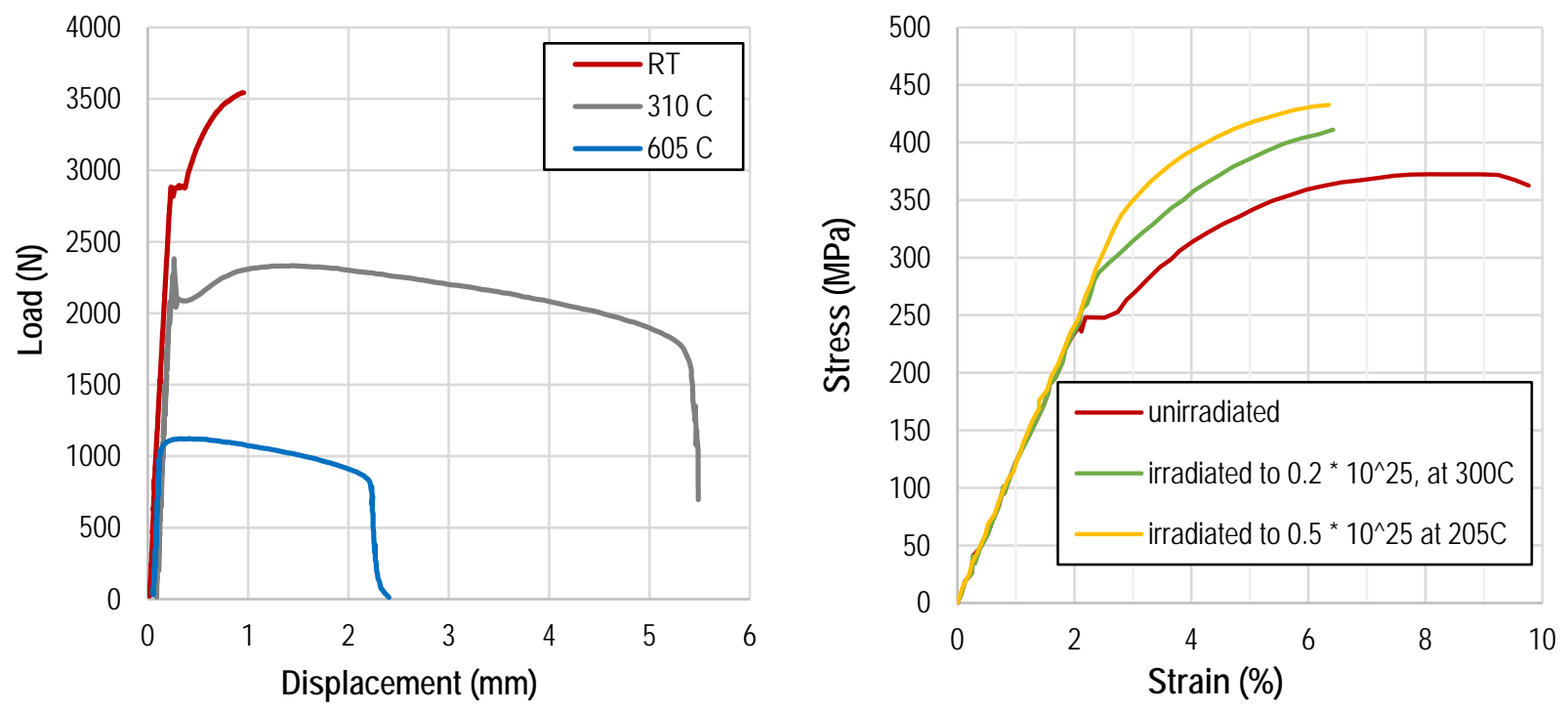

Figure 3.16. Raw data from tensile tests of S-200-FH grade, digitized from (left) and stressstrain curves from tensile tests of $S-65-C$ grade, digitized from (right)

Figure 3.17 and Figure 3.18 show the dependency of yield strength and UTS on temperature for S-200-FH and S-200-F grades of beryllium [47]. Also, such dependency is presented for irradiated samples up to a fluence ( $\mathrm{E}>1.0 \mathrm{MeV}$ ) of $0.15^{*} 10^{22} \mathrm{n} / \mathrm{cm}^{2}$. At that fluence level, there is virtually no difference between the yield and UTS (the solid red line overlaps the dashed red line). While the values of total elongation and UTS will be highly dependent on the grade of the beryllium and exact irradiation and testing conditions, qualitatively it can be concluded that the initial gain in UTS of irradiated beryllium will be accompanied by loss of ductility and embrittlement. At a certain, relatively low, level of irradiation, the ductility will be entirely lost and the failure of a sample tested in tension will occur in the elastic region through a brittle fracture. Subsequent irradiation will result in a decrease of UTS. The goal of this section is to produce a conservative envelope of the UTS as a function of irradiation dose that could be used to estimate the strength of the MURR beryllium reflector as a function of operation time within the reactor (or as a function of irradiation dose). 


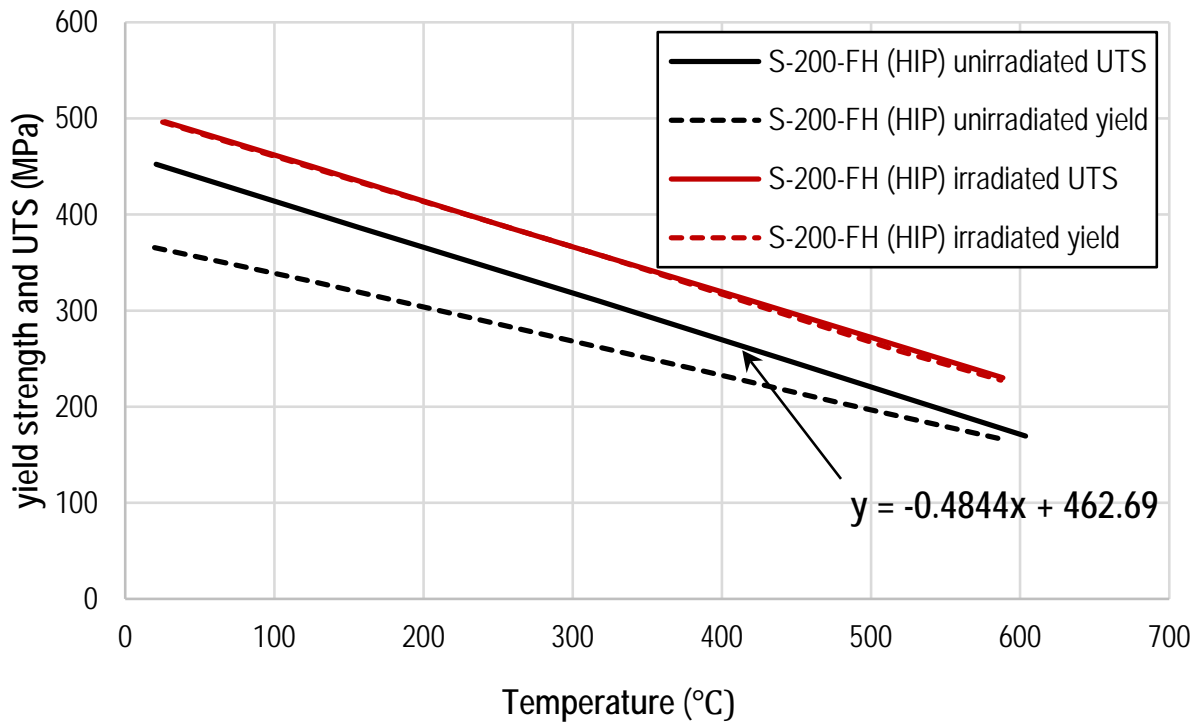

Figure 3.17. Dependency of yield strength and UTS of S-200-FH grade beryllium on temperature for unirradiated and irradiated samples, with irradiation up to a fluence (E > $1.0 \mathrm{MeV}$ ) of $0.15^{*} 10^{22} \mathrm{n} / \mathrm{cm}^{2}[47]$.

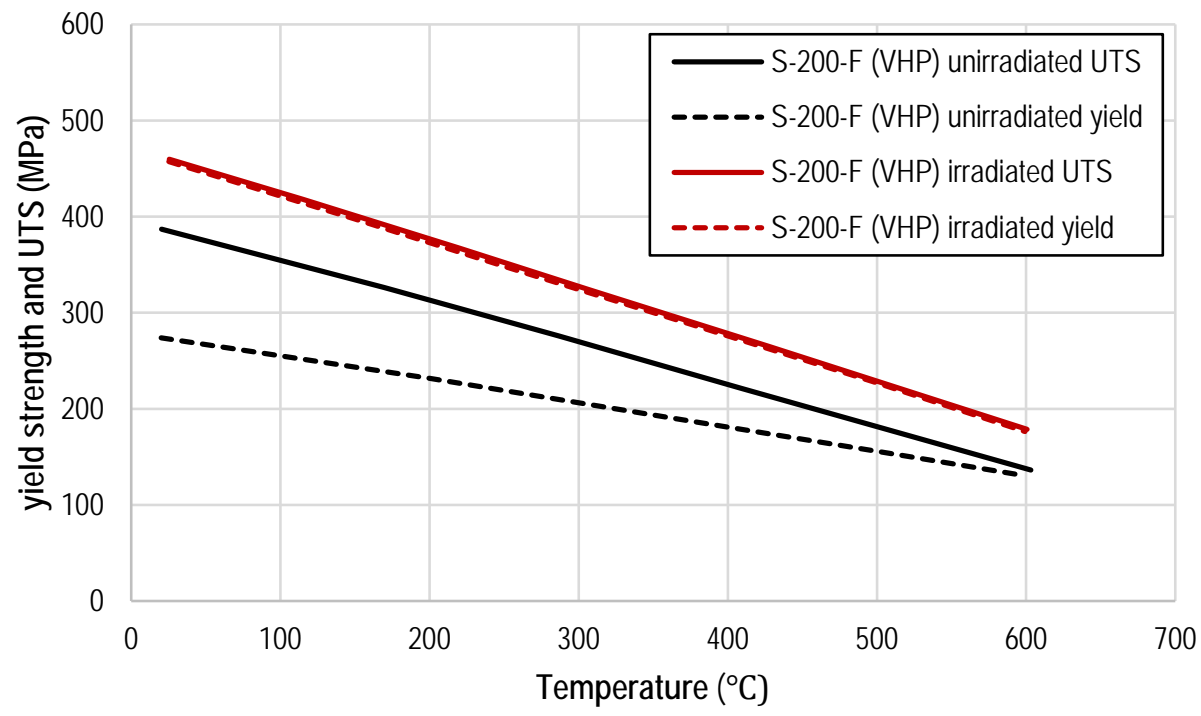

Figure 3.18. Dependency of yield strength and UTS of S-200-F grade beryllium on temperature for unirradiated and irradiated samples, with irradiation up to a fluence (E > $1.0 \mathrm{MeV}$ ) of $0.15^{*} 10^{22} \mathrm{n} / \mathrm{cm}^{2}$.

The most extensive data on yield strength in tension $\left(\sigma_{\mathrm{yt}}\right)$ and UTS of neutron-irradiated beryllium at low temperatures come from three sources, i.e., the ATR [8], [68], [69], BR2 [63], [47]and SM [12], [13], [70], [71] reactors.

Figure 3.19 presents the data obtained at ATR for hot-pressed beryllium [8]. The grade used for the testing was not specified except for the symbol QMV, which was a brand name for a vacuum hotpressed beryllium produced in the 1950s through 1970s by Brush Beryllium Company [72] (which became Brush Wellman in 1971 [73] and later became Materion). QMV included N-200-A grade 
beryllium, used for the first and second MURR reflectors, as well as early S-100, S-200 series and other structural grades produced at the time. For that reason, from the openly available literature, it is not possible to determine which grade was tested by Beeston at the time [8].

Additional data points (represented in Figure 3.19 by points with white filling), based on data from [68] and [69], from tensile tests of QMV beryllium have been added to this figure. The unirradiated tensile strength of $241 \mathrm{MPa}(35 \mathrm{ksi}$ ) reported in these references indicates that these could have been data for grade S-100-C, N-50-A, or N-100-A. All of these grades had significantly lower ductility, yield, and UTS than the current S-200-F and S-200-FH grades. Therefore, it is assumed that the structural performance (characterized here by ductility and UTS) of the newer grades produced nowadays by Materion, like S-200-F and S-200-FH, is not lower than the strength of these historical grades.

The results of three different methods of testing beryllium in tension are presented in Figure 3.19. The samples exposed to low irradiation levels were tested in a direct uniaxial tension test. The use of the direct tensile test for highly irradiated beryllium samples is problematic because of their high brittleness. The samples need to be clamped in the apparatus grips, which may cause their rupture before the test even begins. Thus, indirect methods of testing (flexure and splitting) were used. It can be noted that not only did the strength of the samples vary between the two tests, but also a large spread of the data was reported within each method of testing. In the end, Beeston [8] recommended the averaged fitted linear function, the line represented by the black continuous line in Figure 3.19, to predict the UTS of irradiated beryllium. This line seems to be an extension of the irradiated ultimate strength data from the direct uniaxial tensile test.

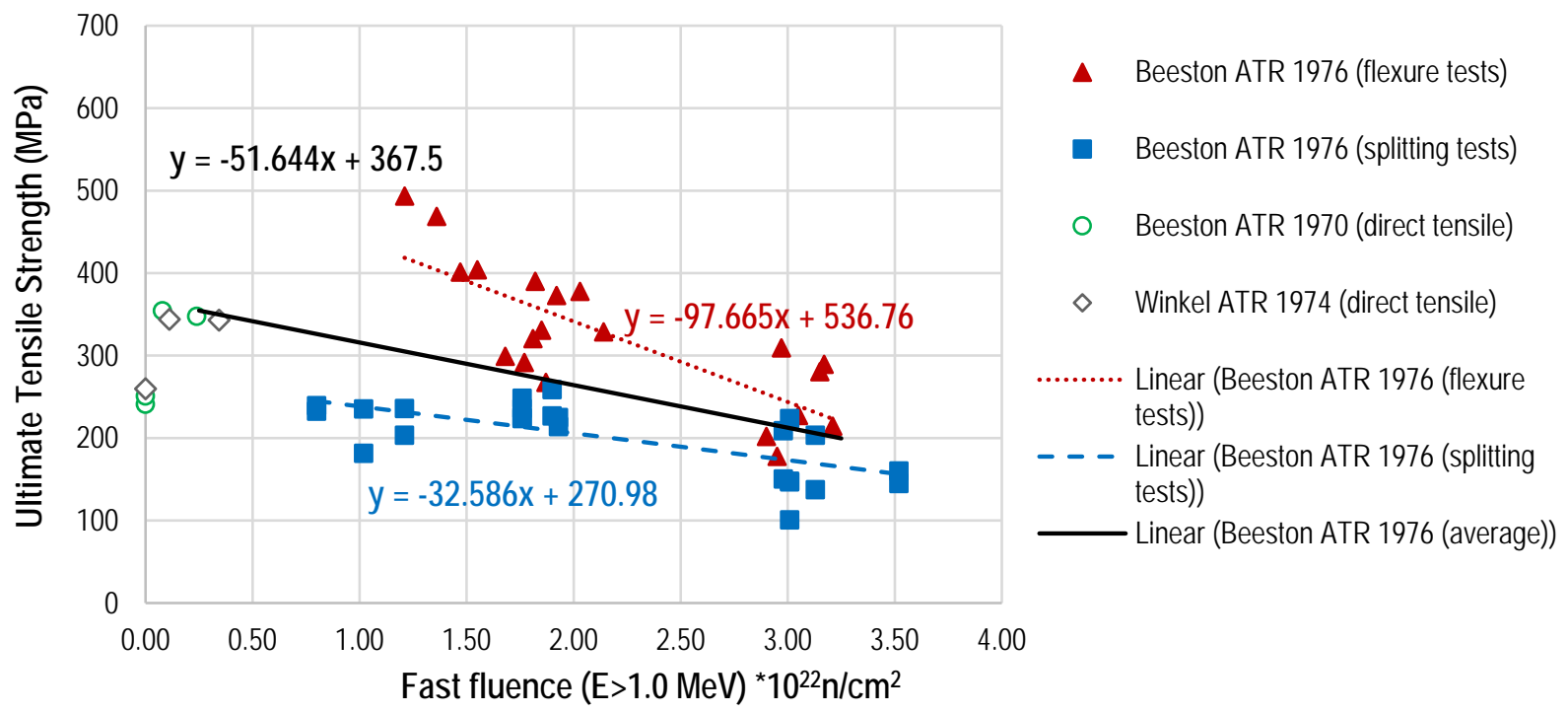

Figure 3.19. Tensile yield strength and UTS data following irradiation at ATR

Several grades of irradiated beryllium were tested in tension and compression at the SM reactor [12], [13], [70], [71]. The majority of tension test results from these references are presented in Figure 3.20. The characteristics (chemical composition, grain size, and production technology) of the TE, TIP and DIP grades can be found in [49] and [74]. The characteristics of TV grades of beryllium are not clear from these references. It appears from [73] and [75] that TV is an alternate name for TE grades. Nevertheless, the original names from the references have been retained here. 
It can be seen from Figure 3-19 that at comparable levels of fluence, the measured values for UTS from SM tend to be closer to (or even lower than) the splitting-test results from ATR (compare Figure 3.19 and Figure 3.20). The samples irradiated at the SM reactor were dumbbell-shaped and were tested via a direct tension procedure [12]. Also, it can be noted that the spread of the data is very substantial. Compared to the thermal conductivity or fracture toughness, the UTS appears to depend significantly more on the grade of the beryllium material.

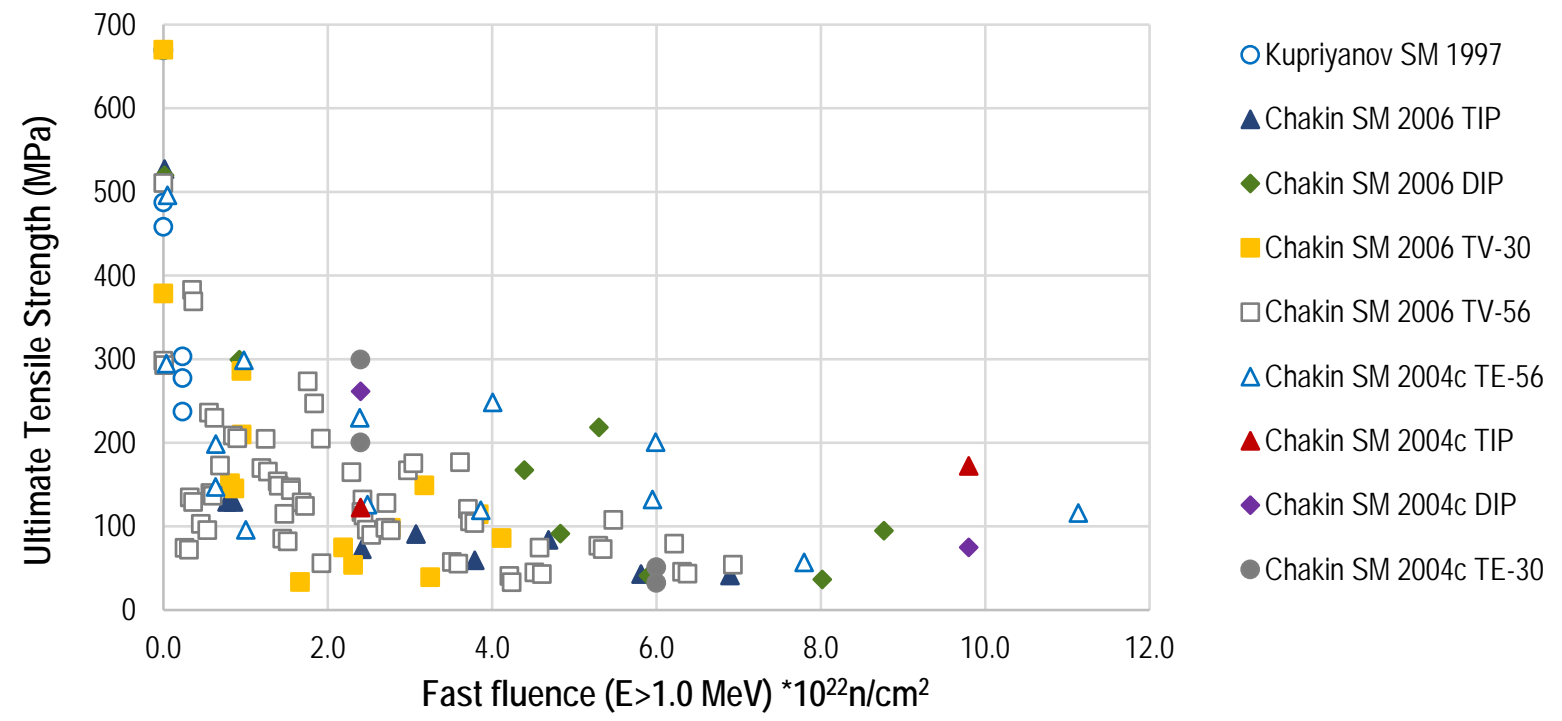

Figure 3.20. UTS data for various beryllium grades tested at the SM reactor

Figure 3.21 presents only the data for grades produced by the HIP method, with their UTS shown as a function of damage. The data show that the strength degradation of irradiated beryllium material is not a strong function of the method of production. Although the two grades shown here had a similar strength in the fresh state, $>500 \mathrm{MPa}$, the strength of the TIP grade degraded much faster than the strength of the DIP grade. The two grades' differences in chemical composition [55] could have influenced the observed difference in UTS behavior. 


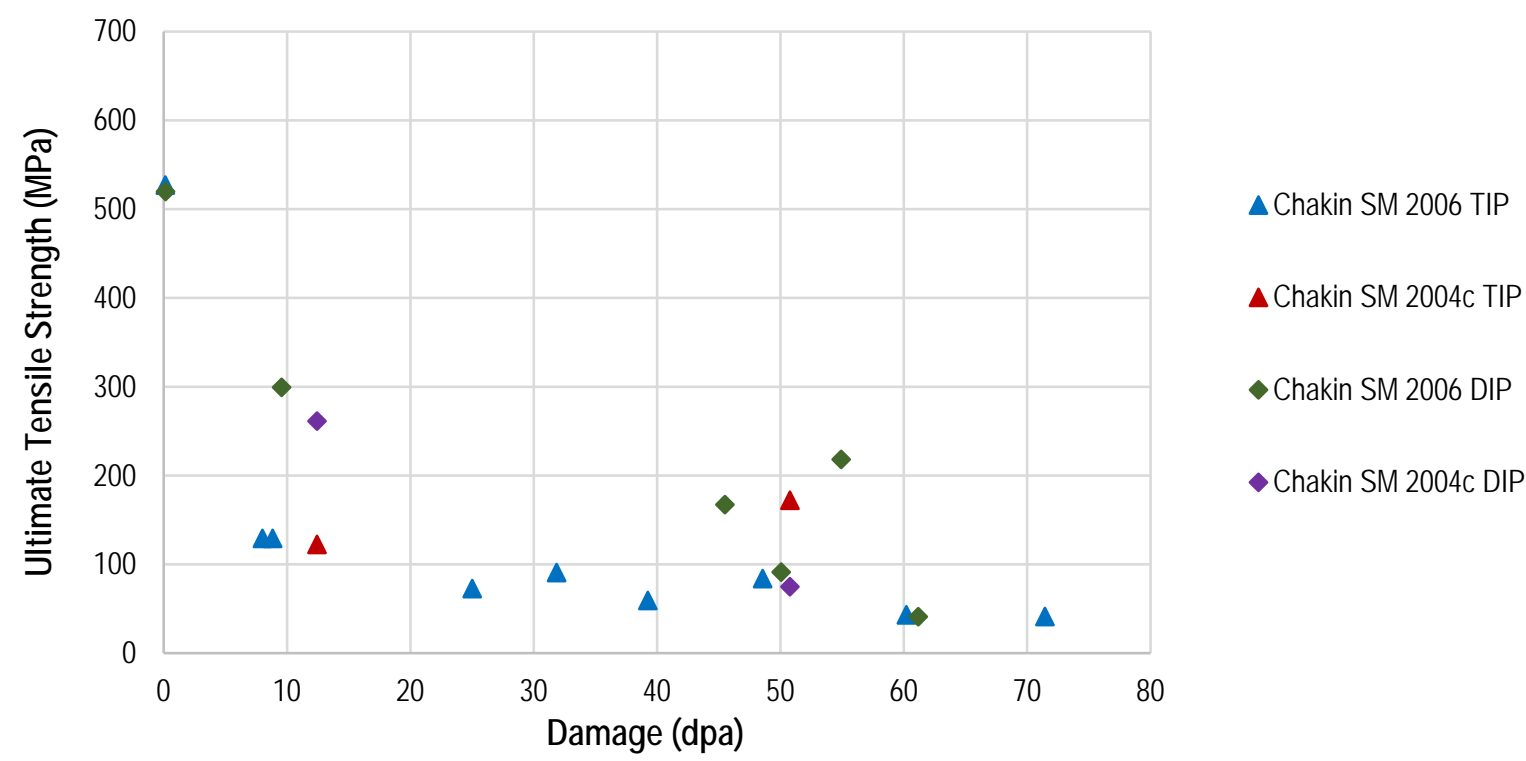

Figure 3.21. UTS data for HIP beryllium grades tested at SM reactor

After 8 years of operation, FE simulations have predicted that the largest tensile stresses of the reflector are on its outer surface near the mid-height behind the spacer grooves. As presented in Section 2.3, the damage at that location after 8 years of operation is equal to $4.4 \mathrm{dpa}$ and $4.7 \mathrm{dpa}$ for HEU and LEU cores, respectively. At a hypothetical 12 years of operation, the damage on that outer surface would reach 6.5 and $7.1 \mathrm{dpa}$ for HEU and LEU cores, respectively. On the inner surface of the reflector, where the damage is over three times as high, only compressive stresses are present. Thus, to predict the life of the MURR beryllium reflector as determined by the tensile stresses and degraded strength of beryllium in tension (refer to Section 3.5), the UTS degradation function needs to cover only the range of damage up to about $8 \mathrm{dpa}$.

Figure 3.22 presents another set of data for UTS obtained at BR2 (SCK) for S-200-FH grade (the grade currently used at MURR) produced by Materion, denoted by yellow and blue circles. The UTS for this material at low levels of damage $(\sim 0.25 \mathrm{dpa})$ for some measurements is greater than the minimum UTS of $414 \mathrm{MPa}$ assumed in this work (see Table 3.1), with a spread from about $350 \mathrm{MPa}$ to almost $500 \mathrm{MPa}$. The samples tested at SCK were irradiated at temperatures of $185^{\circ} \mathrm{C}$ or $235^{\circ} \mathrm{C}$ and tested at $25^{\circ} \mathrm{C}, 185^{\circ} \mathrm{C}$, and $235^{\circ} \mathrm{C}$. Thus, the samples of S-200-FH tested at $25^{\circ} \mathrm{C}$ may be underpredicting its strength, while the samples tested at around $200^{\circ} \mathrm{C}$ may be overpredicting its strength at $\sim 70^{\circ} \mathrm{C}$. Unfortunately, the present authors could not find any more data points for that particular grade in the available literature, and assumptions regarding the degradation of the S-200-FH due to irradiation damage had to be made.

UTS data for unirradiated S-200-FH (triangles and squares), together with data points for DIP grade from SM reactor tests, are also presented in Figure 3.22. This set of data was chosen to build the UTS degradation function for S-200-FH. Polynomial-based fits to those data were performed and are presented in Figure 3.23. The starting point for the fit for S-200-FH was forced at $430.3 \mathrm{MPa}$, which corresponds to the UTS of an unirradiated sample tested at $65^{\circ} \mathrm{C}$. Also included in the plot are the lower $95 \%$ confidence interval to the mean fit as well as various confidence intervals for individual samples. A proposed fit for the UTS of N-200-A grade is also included. 


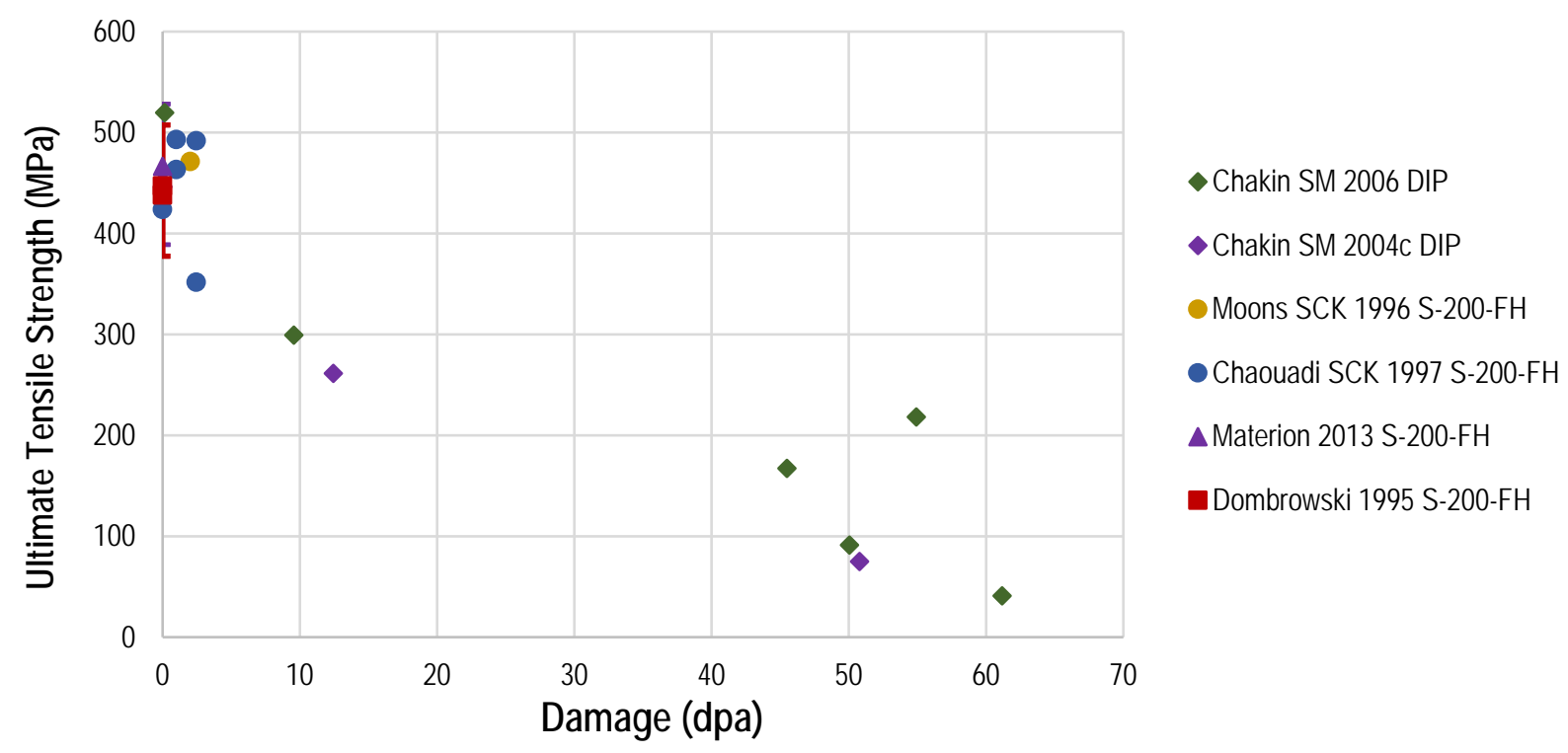

Figure 3.22. UTS data for HIP beryllium grades tested at BR2 and SM reactors as a function of damage

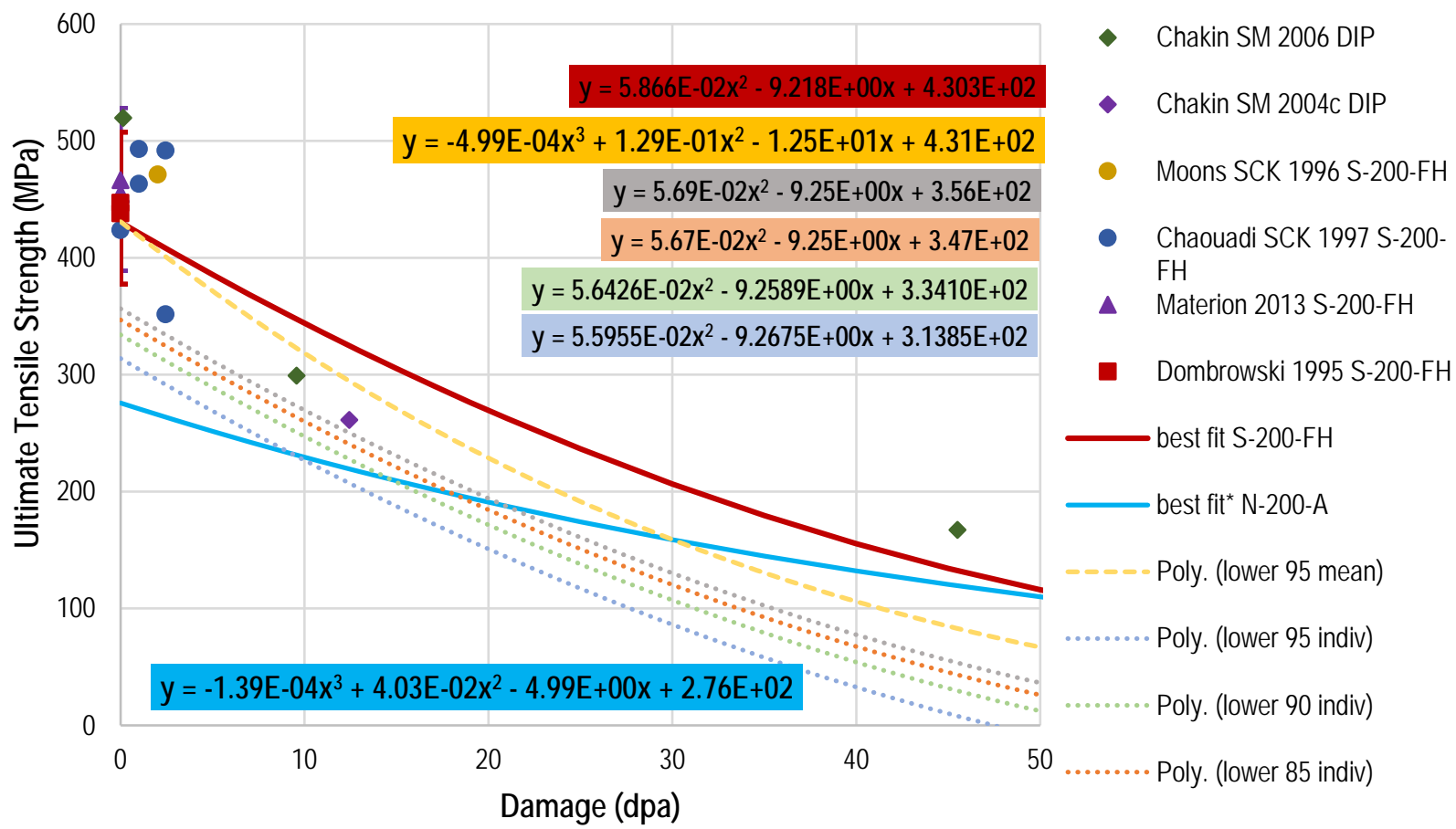

Figure 3.23. UTS degradation data with an assumed fit for S-200-FH and N-200-A grade beryllium

Figure 3.24 shows the data from ATR (presented previously in Figure 3.19) with the assumed UTS degradation model for S-200-FH grade. Since there is no reliable conversion from fluence to dpa for the data obtained at ATR, here the UTS data are presented as a function of fluence, noting that the two reactors may have different energy spectra. 
The proposed UTS degradation model for S-200-FH corresponds well with the averaged linear fit to the data from ATR (black line in Figure 3.19 and Figure 3.24). Since the beryllium grade used for testing at ATR was produced by Materion, and is similar to the grade that was used for the MURR reflector before HIP technology was introduced by Materion in 1985, the assumed degradation model is considered conservative for estimations of the lifetime of MURR beryllium reflectors.

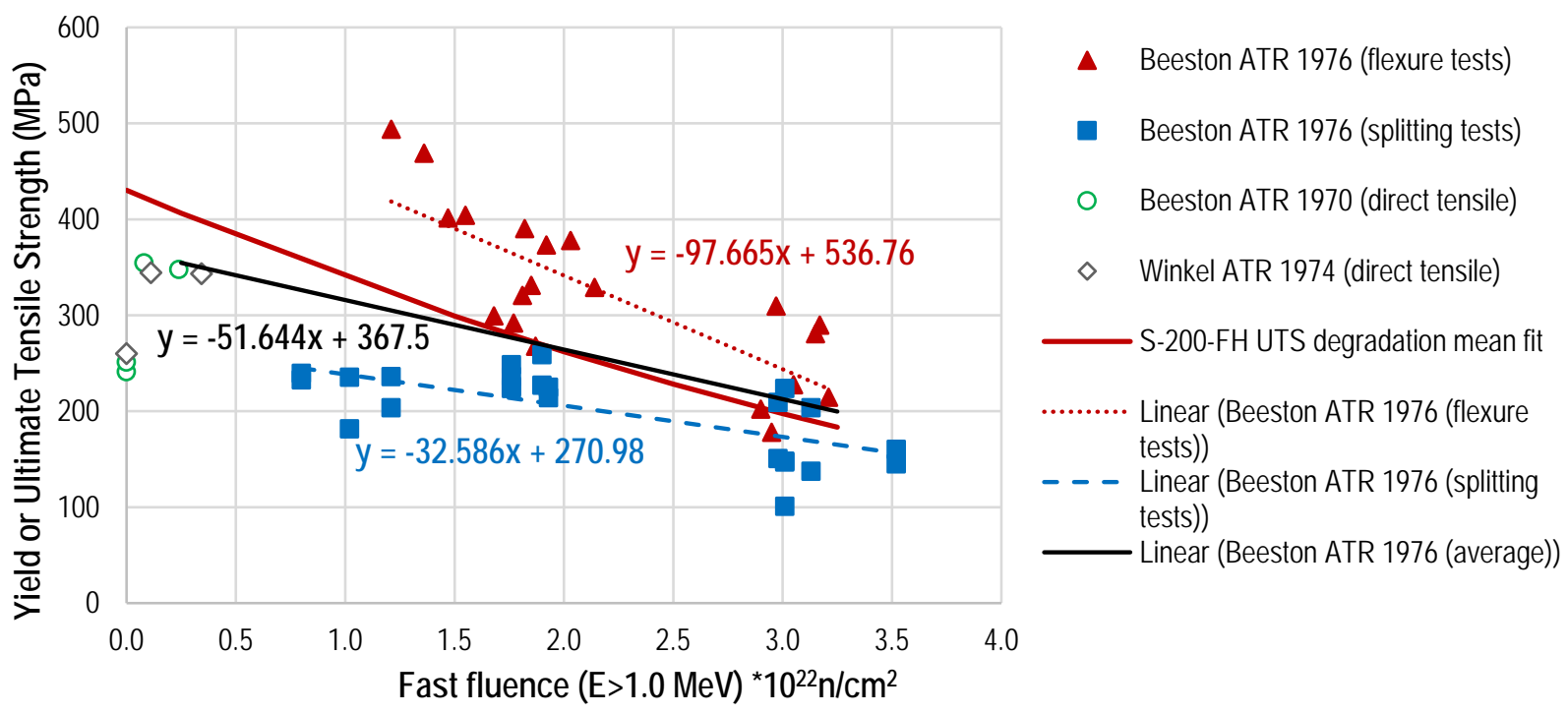

Figure 3.24. Tensile yield strength and UTS data following irradiation at ATR with assumed UTS degradation model for S-200-FH

Figure 3.25 and Figure 3.26 present the distribution of estimated UTS in a reflector made of S-200-FH at 8 years of operation for the MURR HEU and LEU cores, respectively. The distribution is based on the UTS degradation model and the damage distribution presented in Section 2.3.
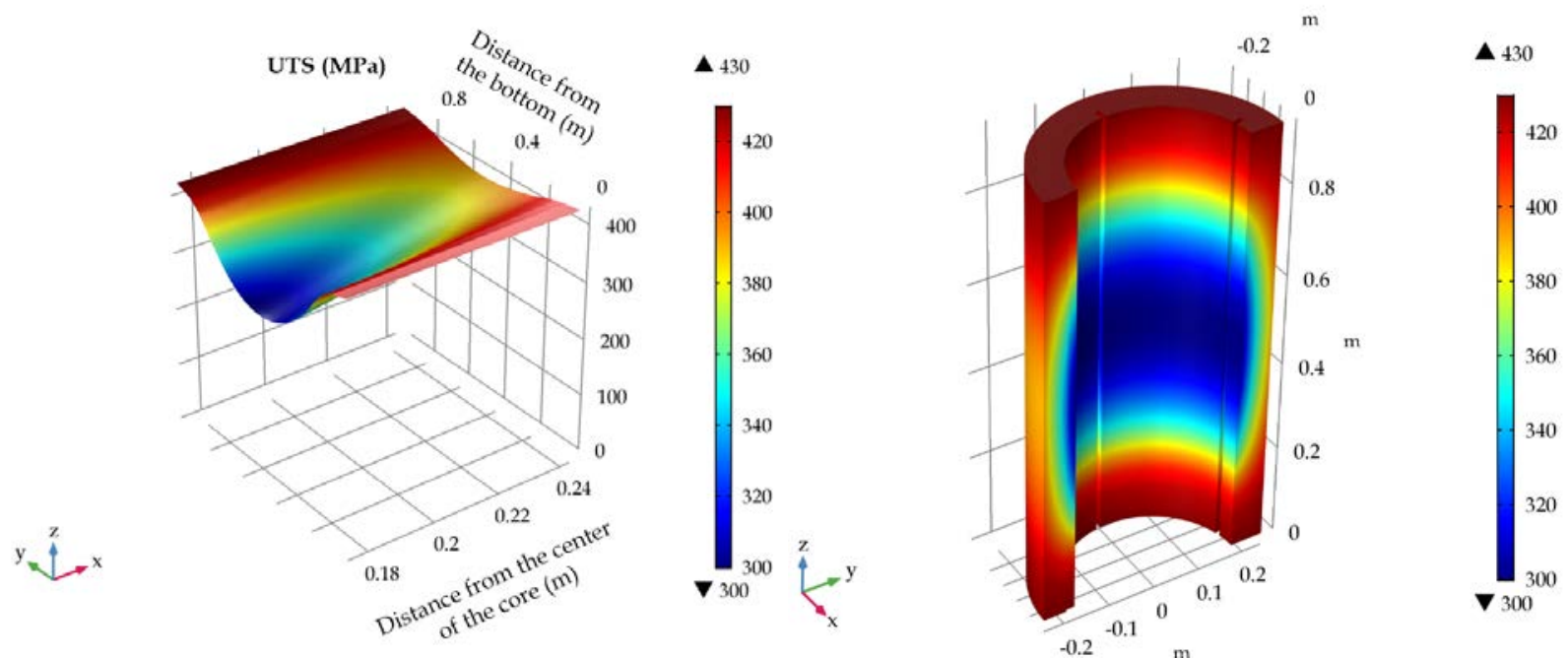

Figure 3.25. UTS (MPa) in S-200-FH beryllium reflector irradiated for 8 years with HEU core. Damage-dependent fit function implemented in COMSOL (left); data extrapolated on the beryllium model (right). 

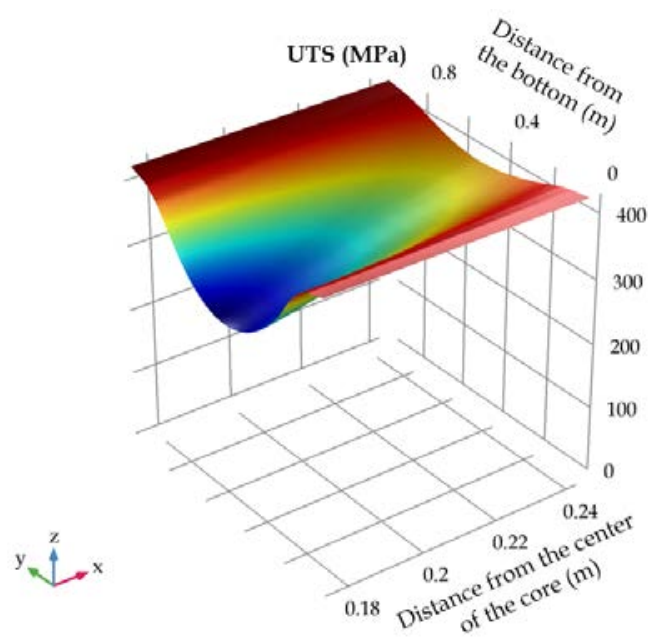
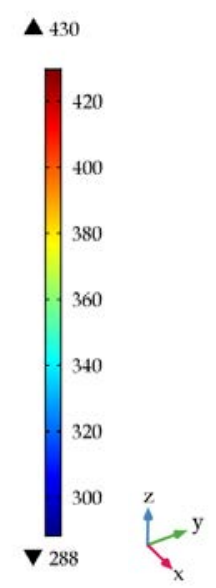

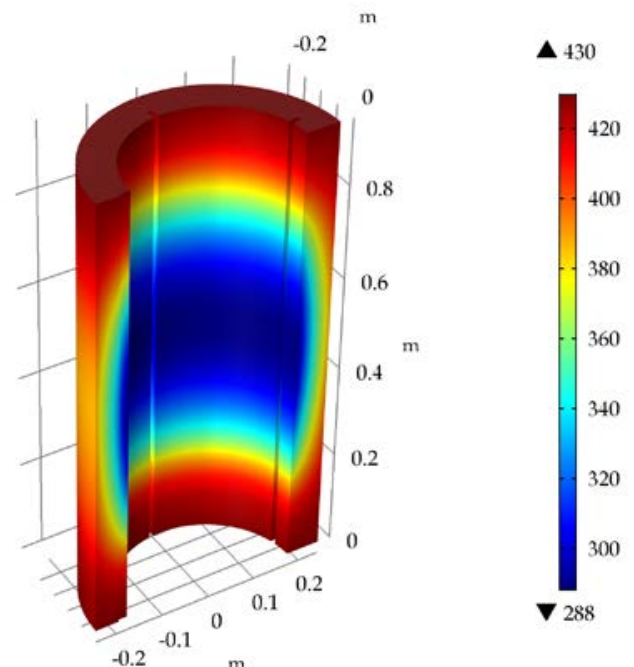

$-0.2-0.1-\mathrm{m}$

420

400

380

60

320

300
Figure 3.26. UTS (MPa) in S-200-FH beryllium reflector irradiated for 8 years with LEU core. Damage-dependent fit function implemented in COMSOL (left); data extrapolated on the beryllium model (right).

\subsubsection{Compressive Strength}

Figure 3.27 presents data for yield strength in compression $\left(\sigma_{\mathrm{yc}}\right)$ and UCS for two samples of beryllium. The availability of these data is unique because usually only one of the two ( $\sigma_{\mathrm{yc}}$ or UCS) is reported in the literature. Very few sources present data for both for the same specimens. The data coming from the Materials Testing Reactor (MTR) and SM reactors are presented as a function of fluence. While the samples of beryllium that were tested are not representative of the material used at MURR, the data show that the difference between $\sigma_{\mathrm{yc}}$ and UCS decreases with irradiation and that the two become essentially equivalent at neutron fluence levels that are expected in the MURR reflector at 8 years of operation $\left(1.4^{*} 10^{22} \mathrm{n} / \mathrm{cm}^{2}\right.$ to $0.4^{*} 10^{22} \mathrm{n} / \mathrm{cm}^{2}$ through the thickness at midheight, as shown in Figure 2.3). For fresh historical QMV beryllium, Beeston [8] reports a yield strength of about $220 \mathrm{MPa}$. Kupriyanov et al. [71] report a yield strength between $398 \mathrm{MPa}$ and 560 MPa for newer TE-30, TIP-30, and TshG-56 grades. The UCS of fresh beryllium is reported to be 1,340 MPa for QMV beryllium (MTR data) and from 1650 to $1810 \mathrm{MPa}$ for SM data (TE-30, TIP-30, and TshG-56 grades). These values correspond well with the UCS of fresh S-200-FH presented in Figure 3.1. With increasing fast neutron $(\mathrm{E}>1.0 \mathrm{MeV})$ fluence, the yield strength increases while the UCS decreases. At about $0.33 * 10^{22} \mathrm{n} / \mathrm{cm}^{2}$, there is no ductility left (no yielding) in the beryllium and the yield-strength concept is meaningless; at that fluence, the samples start to experience brittle failure. 


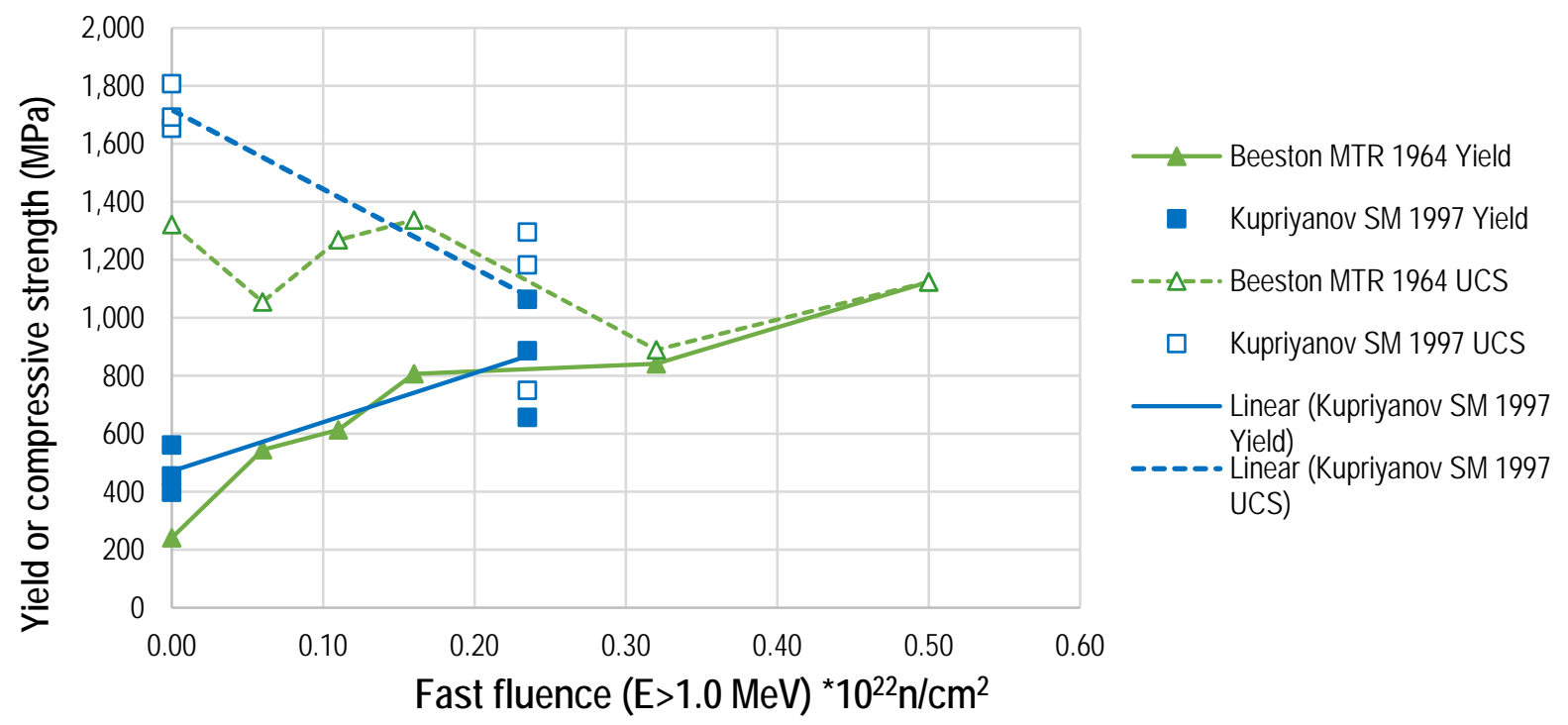

Figure 3.27. Yield strength and UCS for fresh and irradiated beryllium material, based on data from MTR and SM

Data from Figure 3.27 are also presented in Figure 3.28 together with additional, more extensive UCS data from ATR reported by Beeston [8]. Plotted together, these data indicate that UCS (as measured at MTR and SM) initially decreases with increasing fluence; then at about $0.33 * 10^{22} \mathrm{n} / \mathrm{cm}^{2}$, when the ductility drops to zero, UCS starts increasing with irradiation to a maximum value of about 1650 $\mathrm{MPa}$ at a fluence of about $1.8^{*} 10^{22} \mathrm{n} / \mathrm{cm}^{2}$, followed by a decrease in UCS with continued irradiation.

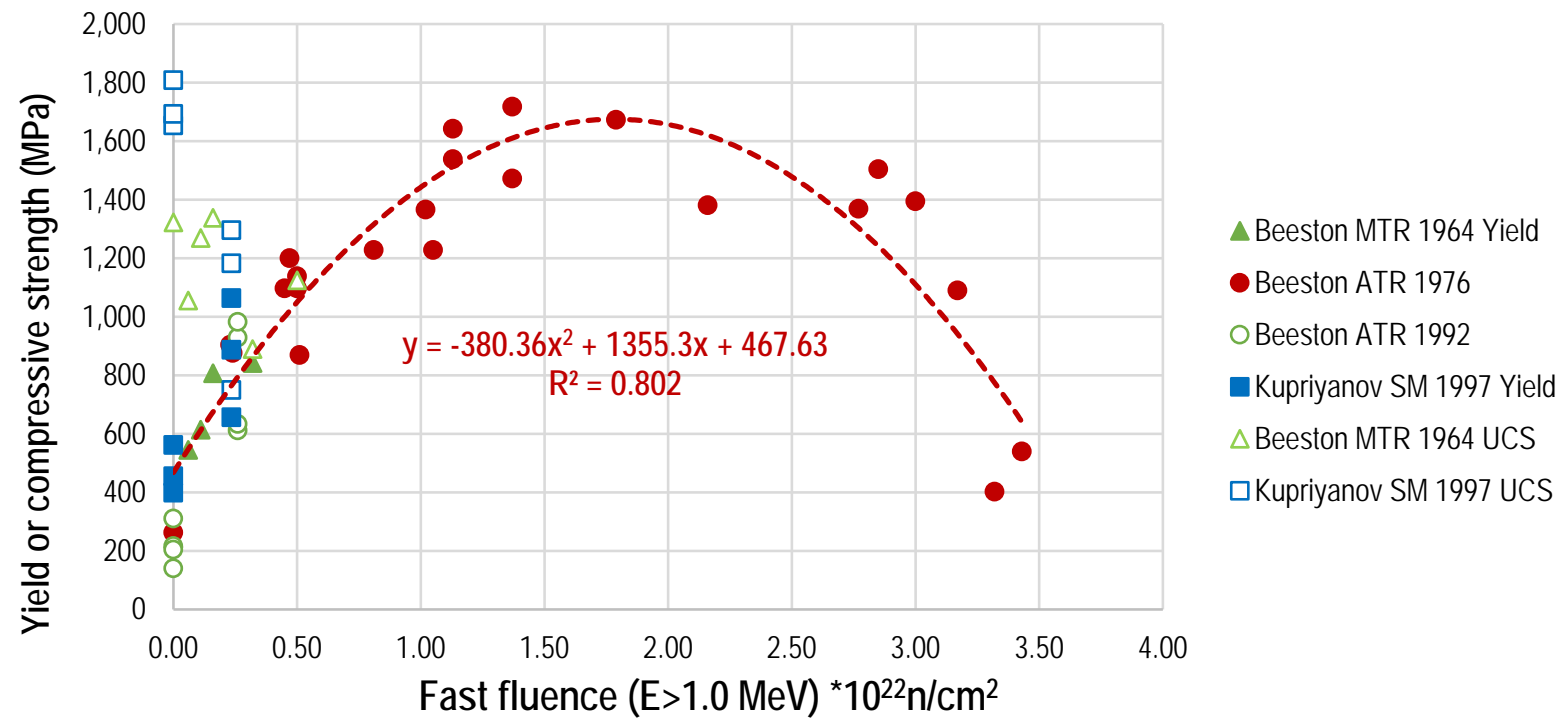

Figure 3.28. Yield or UCS for irradiated beryllium material, based on data from ATR, MTR, and SM

The ATR data, with a peak in strength at a significant level of fluence (or damage), appears to contradict the more recent data presented by Chakin [13] from multiple tests performed at the SM reactor, as shown in Figure 3.29 and Figure 3.30. It is possible that the metrology of both tests influenced the results. Tests performed by Beeston were conducted following the ASTM E9 standard. 
The exact sizes of all the samples were not specified in [8]. However, ASTM E9 requires cylindrical samples subject to compression tests to be $13 \times 25 \mathrm{~mm}$ to $32 \times 320 \mathrm{~mm}$ in diameter and height for the shortest and longest samples, respectively. The size of medium samples, according to ASTM E9, should be in the range from $13 \times 38 \mathrm{~mm}$ to $30 \times 85 \mathrm{~mm}$. The compression tests conducted by Chakin [13] were conducted on cylindrical samples $6 \times 8 \mathrm{~mm}$ in size, which were smaller than the smallest samples recommended by ASTM E9.

Figure 3.29 and Figure 3.30 show the same data, with UCS as a function of fluence and damage (dpa), respectively. For multiple tested grades, Chakin found the UCS to be monotonically decreasing, with the maximum UCS occurring for fresh beryllium material. No other source could be located to verify which of these behaviors is representative for the beryllium grade currently used at MURR. For this reason, the more conservative data reported by Chakin with monotonically decreasing UCS will be used for the beryllium life assessment in the current work (see the fourth-order polynomial fit, a black dashed line in Figure 3.31). Figure 3.31 shows only data from the SM reactor, with the polynomial fit in the range of greatest interest for the MURR beryllium reflector (with damage $<20$ dpa).

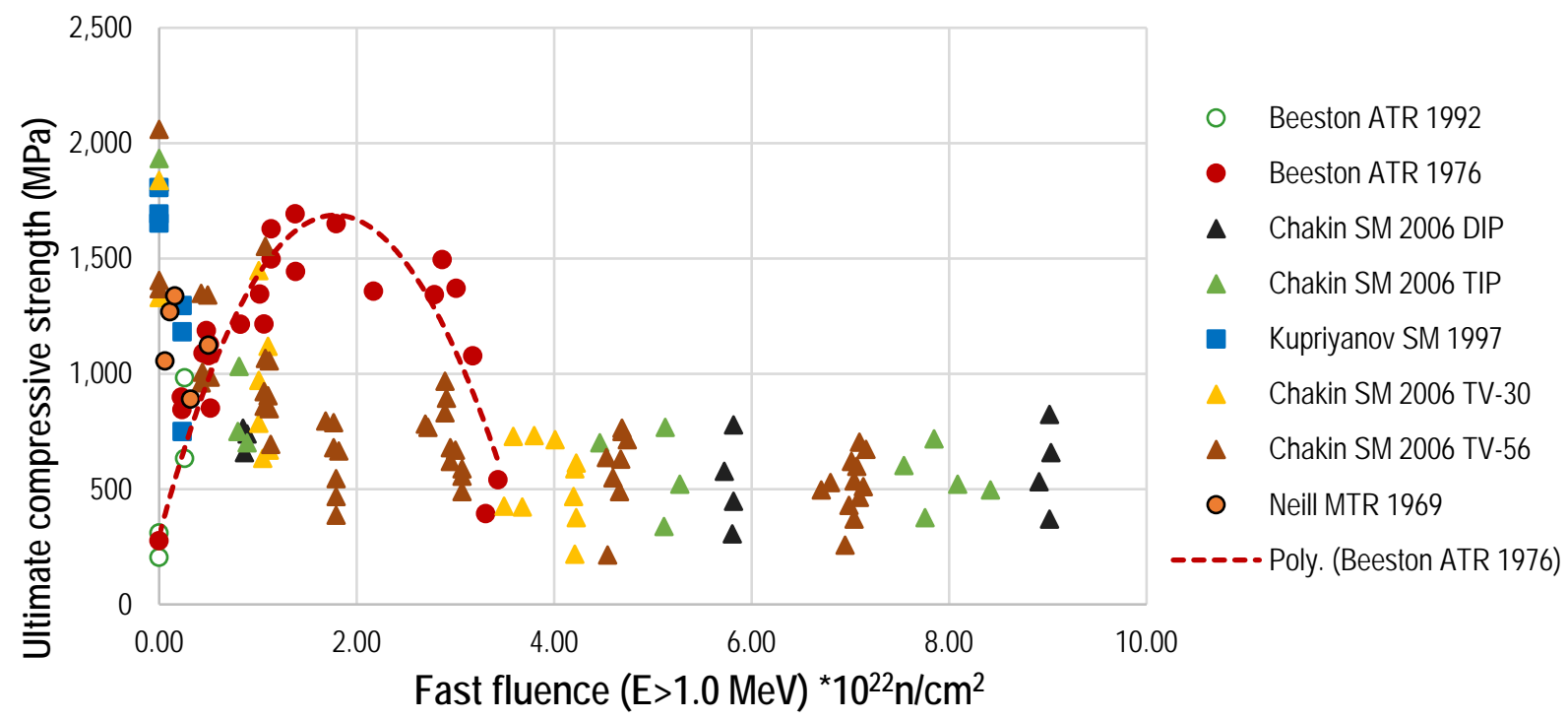

Figure 3.29. UCS for irradiated beryllium material, based on data from ATR, MTR, and SM as a function of fluence 


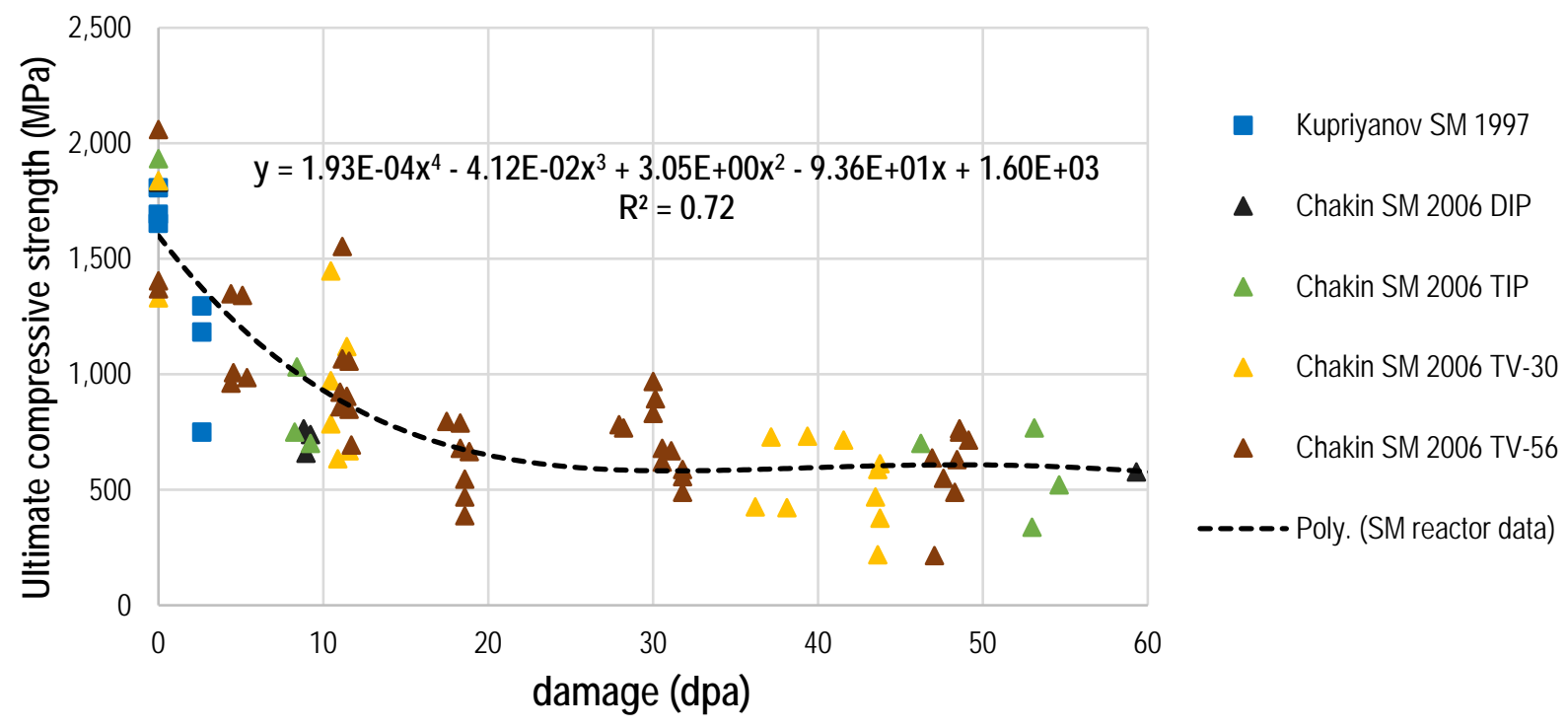

Figure 3.30. UCS for irradiated beryllium material, based on data from SM as a function of damage

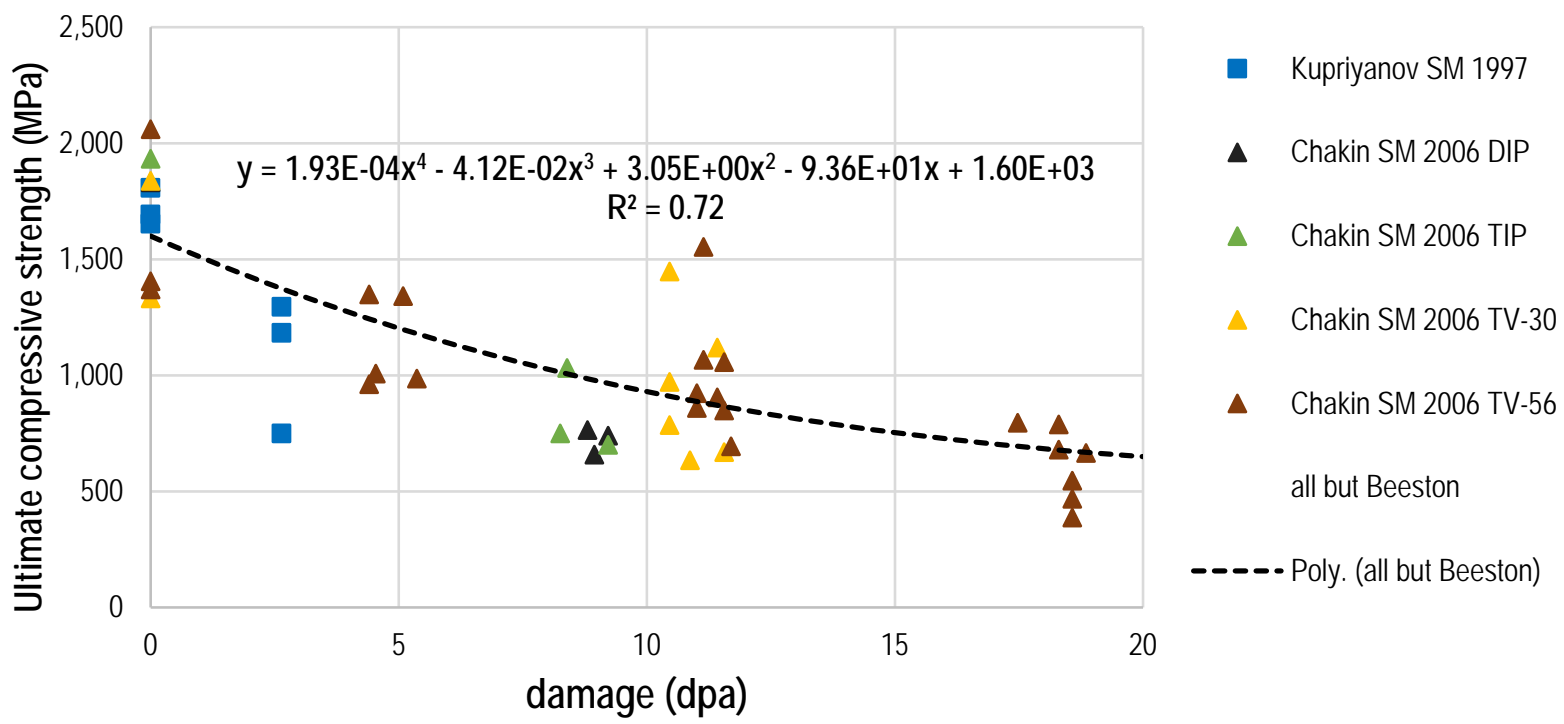

Figure 3.31. UCS for irradiated beryllium material based on data from SM, with the polynomial fit to the data

Figure 3.32 and Figure 3.33 show the damage-dependent UCS fit functions implemented in COMSOL as well as the distribution of UCS in the beryllium models for HEU and LEU cores, respectively, at 8 years of operation. The lowest UCS is calculated for the area with the highest damage and it is roughly half of the UCS of fresh beryllium, which is assumed to be 1,600 MPa. 

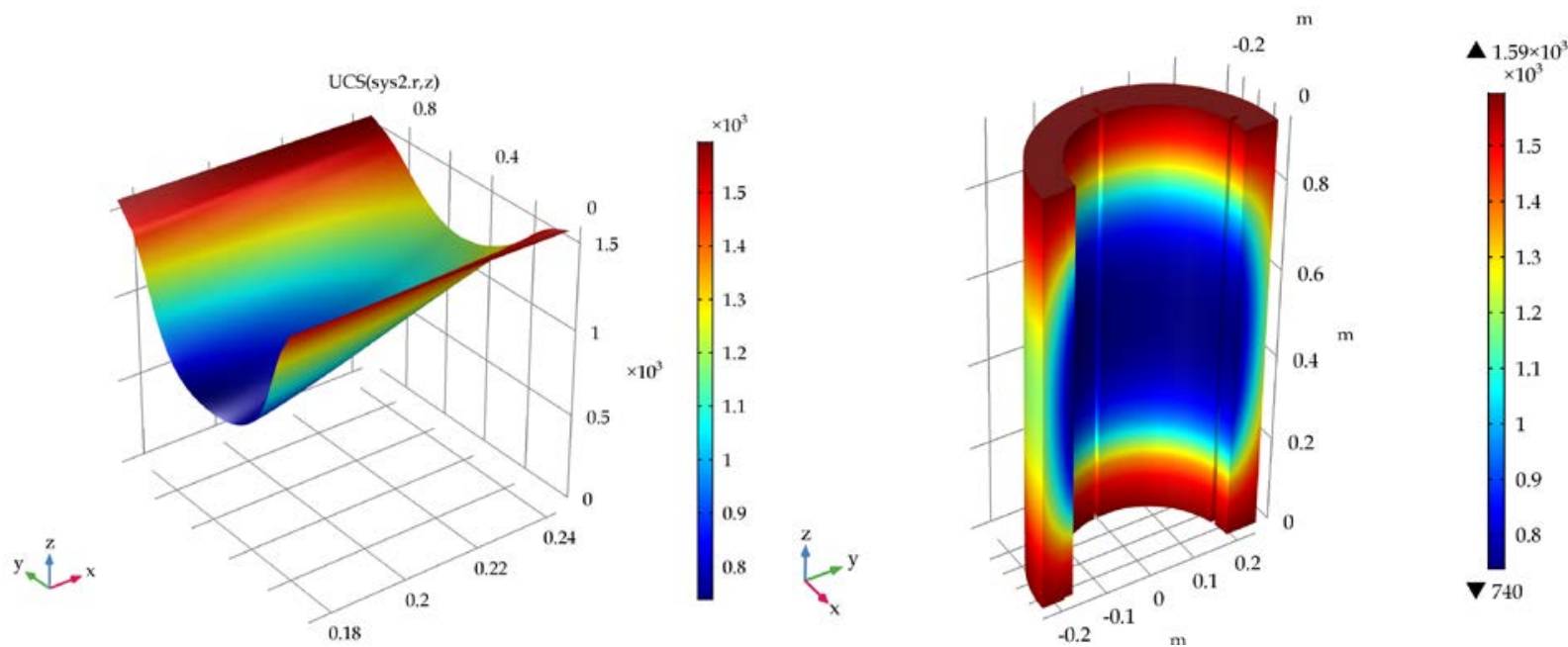

Figure 3.32. UCS (MPa) in beryllium irradiated for 8 years with MURR HEU core. Damagedependent fit function implemented in COMSOL (left); data extrapolated on the beryllium model (right).
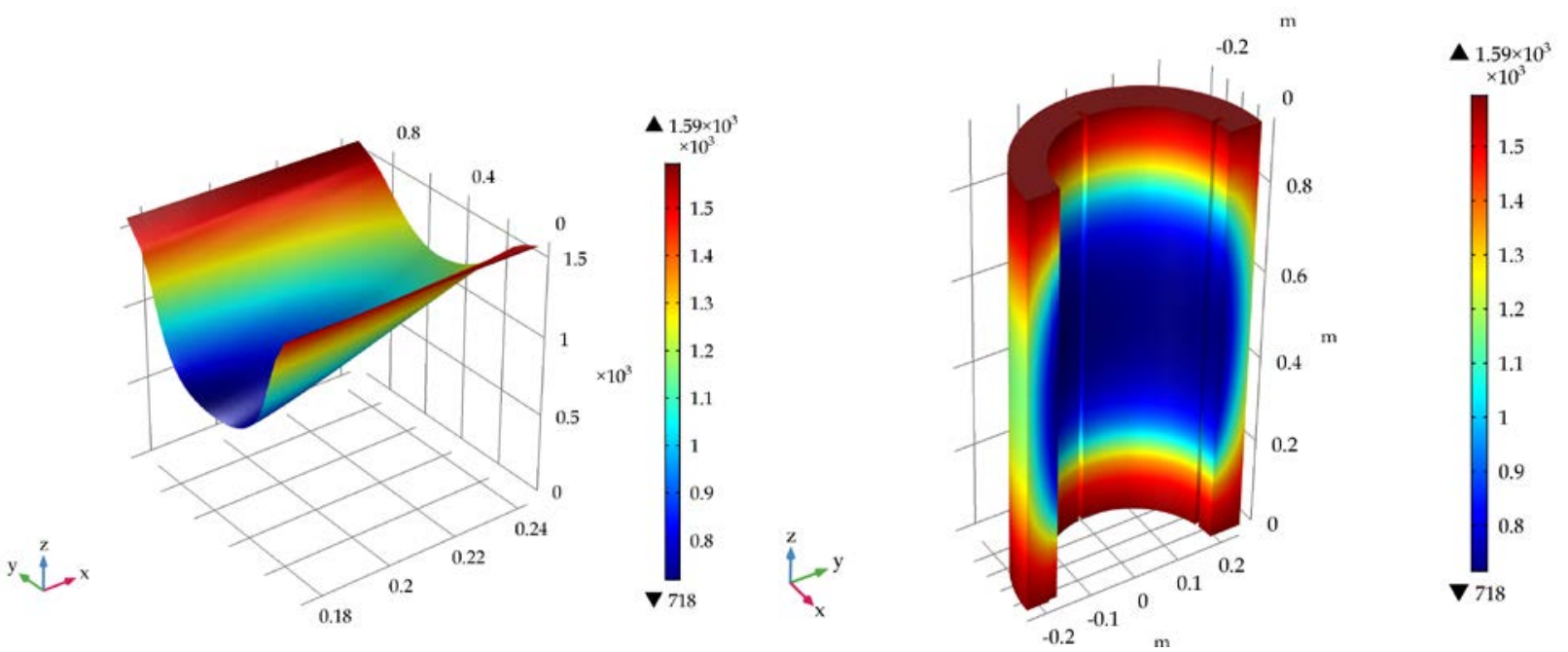

Figure 3.33. UCS (MPa) in beryllium irradiated for 8 years with MURR LEU core. Damagedependent fit function implemented in COMSOL (left); data extrapolated on the beryllium model (right).

\subsubsection{Fracture Toughness}

Beryllium fracture-toughness degradation due to irradiation has not been well studied, especially for a high neutron fluence $(\mathrm{E}>1.0 \mathrm{MeV})$ of $0.5^{*} 10^{-22} \mathrm{n} / \mathrm{cm}^{2}$. There are only two sources of data available for fracture toughness of beryllium irradiated at low temperatures, namely, the data from ATR [76] and SCK [62], [77]. The ATR data were obtained for a historical Kawecki Berylco Industries (KBI) pressed grade. SCK data were obtained for vacuum hot-pressed S-65 and S-200-F, and isostatically hot-pressed S-65 and S-200-FH (as currently used at MURR). Irradiations at SCK were performed at various temperatures from $200^{\circ} \mathrm{C}$ to $610^{\circ} \mathrm{C}$. Here, only the subset of data for lower temperatures from that range $\left(200^{\circ} \mathrm{C}\right.$ to $\left.230^{\circ} \mathrm{C}\right)$ is presented and discussed. It was observed that the material grade did not influence the fracture toughness or the rate of its degradation at these lower temperatures. 
Elevated-temperature $\left(>300^{\circ} \mathrm{C}\right)$ testing indicated that there is an annealing effect on the fracture toughness. However, samples irradiated at $\sim 200^{\circ} \mathrm{C}$ and tested at $\sim 200^{\circ} \mathrm{C}$ had similar fracture toughness to the samples irradiated at $\sim 200^{\circ} \mathrm{C}$ and tested at RT.

Figure 3.34, Figure 3.35, and Figure 3.36 show the fracture toughness of beryllium as a function of fluence, He- 4 content, and damage, respectively. Figure 3.34 and Figure 3.35 were produced using data from SCK and ATR. Figure 3.36 includes only the data from SCK, as no reliable conversion factor from fluence to dpa is available for the ATR data.

It has to be pointed out that the ATR dataset reported here is the only one that reaches irradiation levels equivalent to the fluence at the outer surface of the MURR reflector (fluence [E $>1.0 \mathrm{MeV}] 0.31-$ $0.34 * 10^{22} \mathrm{n} / \mathrm{cm}^{2}$ ). There are no data to allow for estimation of the fracture toughness of the beryllium on the inner surface of the MURR reflector (fluence [E $>1.0 \mathrm{MeV}]$ of $1.3-1.4^{*} 10^{22} \mathrm{n} / \mathrm{cm}^{2}$ ). The preference should be to use the data that are dependent on the damage (dpa) as opposed to the fluence, so the result becomes reactor-independent. The dataset from SCK is the only one that can be used in that form. However, the highest damage level obtained in these tests was only $2.3 \mathrm{dpa}$, as compared to $\sim 5 \mathrm{dpa}$ for the damage on the outer surface of the MURR beryllium reflector at 8 years of operation. For that reason, the fracture-toughness fit was built for the fluence-dependent data, as shown in Figure 3.34.

An additional finding from this review is that the spread in the fracture-toughness data appears not to be correlated with the beryllium grade, as four different grades tested at SCK displayed similar performance in toughness tests. This was not the case for the UTS data, where high dependency on the grade was reported.

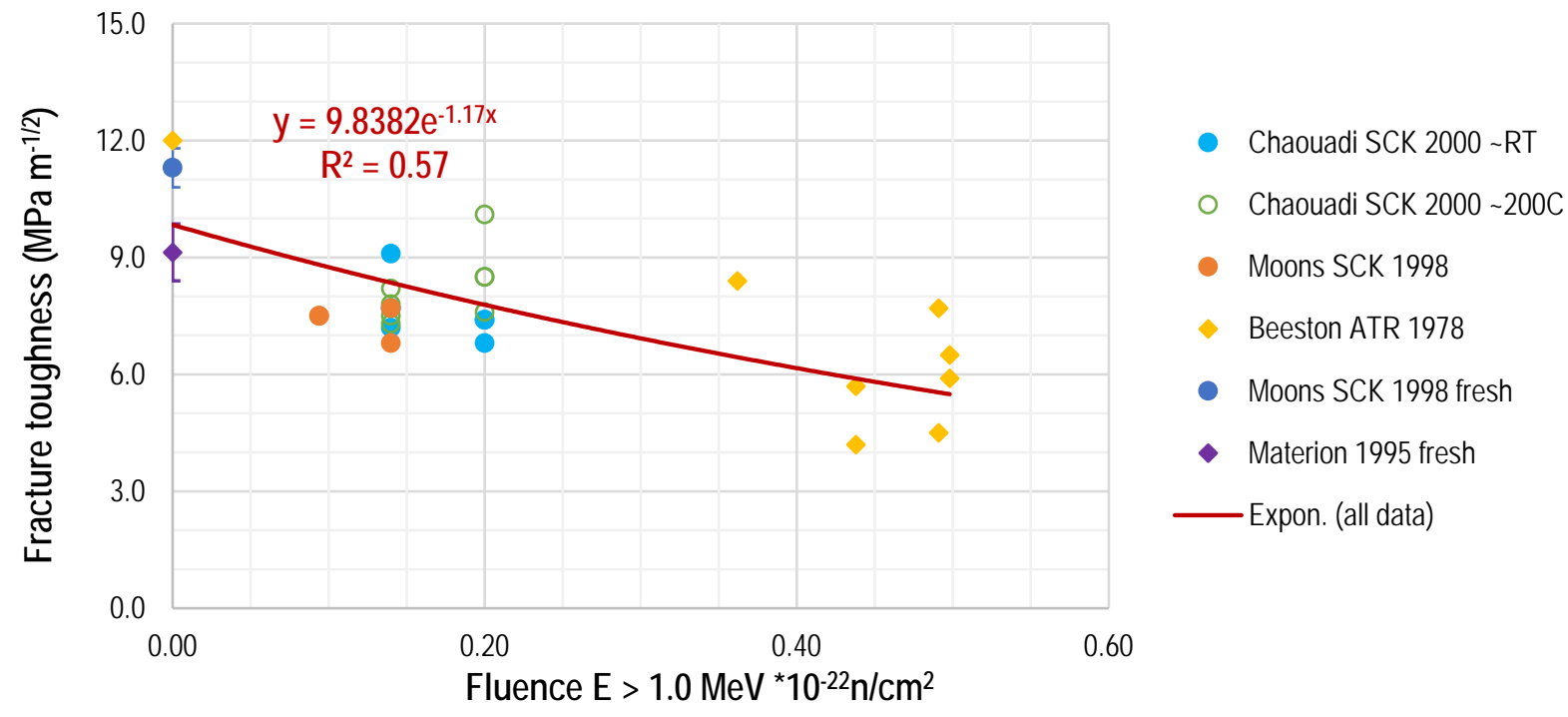

Figure 3.34. Fracture toughness of irradiated beryllium as a function of fluence $(\mathrm{E}>1.0 \mathrm{MeV})$ 


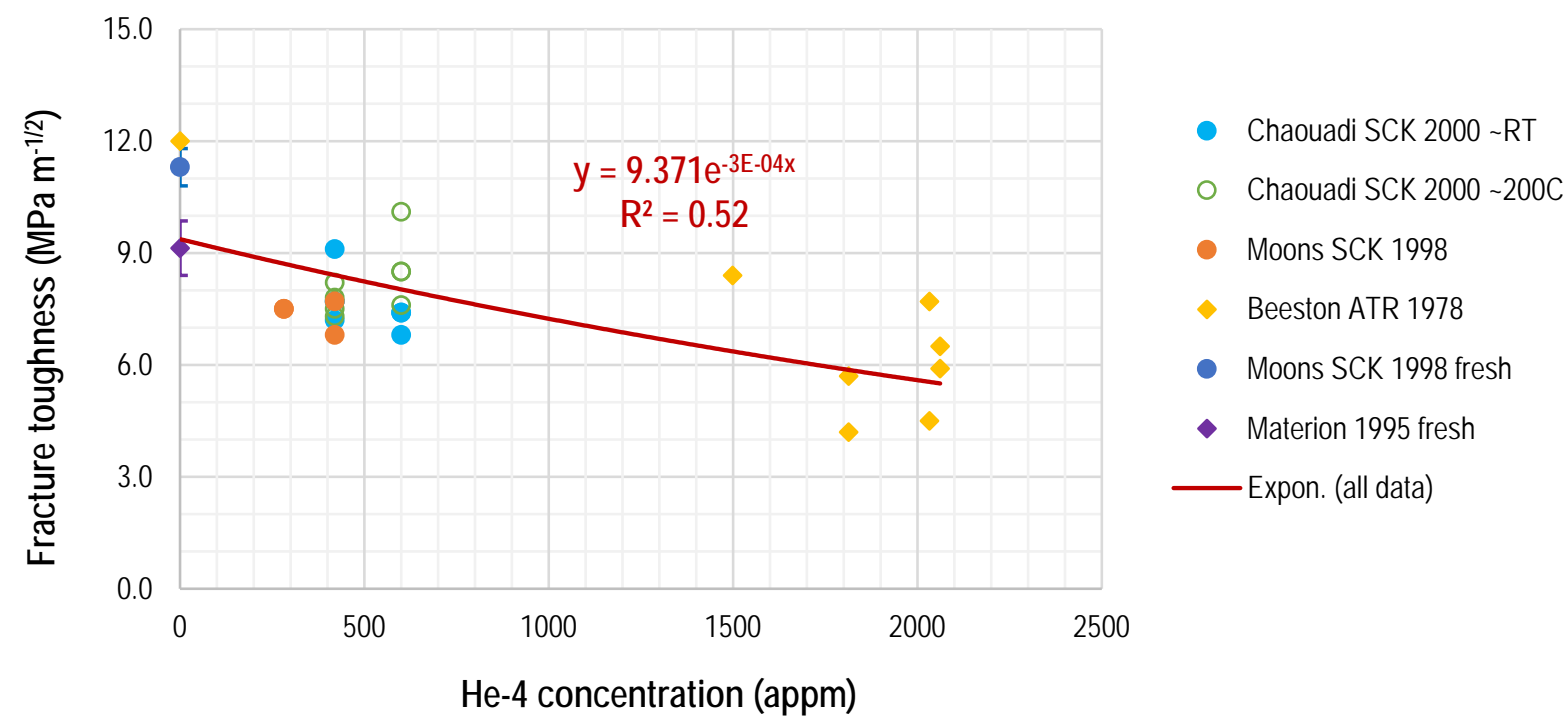

Figure 3.35. Fracture toughness of irradiated beryllium as a function of He-4 accumulation

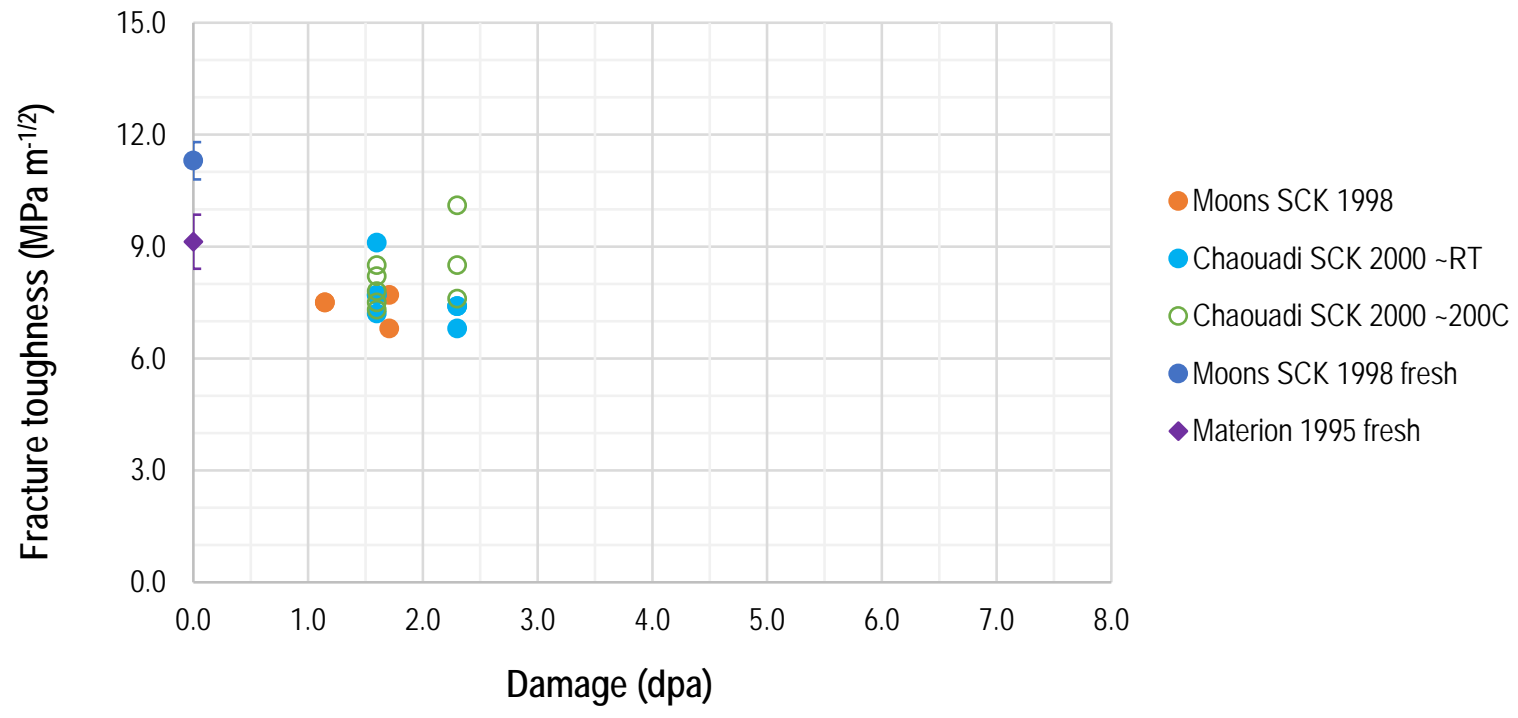

Figure 3.36. Fracture toughness of irradiated beryllium as a function of damage 


\subsection{Volumetric Swelling Model of Irradiated Beryllium}

There are multiple sources of swelling data for irradiated beryllium as well as models used to predict swelling due to irradiation. All of these models are empirically derived fits rather than models based on fundamental theory. This section aims at collecting most current data on swelling of irradiated beryllium and proposing a swelling model that can be used in further FE analysis. For the purpose of this study, the focus was on data that only pertain to beryllium irradiated at low temperatures and tested under similar conditions without (or with minimal) annealing.

As mentioned in Section 3.3, transmutation gases produced under low-temperature irradiation (below $300^{\circ} \mathrm{C}$ ) stay immobile within the lattice of the crystals, and swelling takes the form of so-called solid swelling. This behavior leads to a linear relationship between the helium content and the amount of volumetric swelling [16]. Testing at higher temperatures or annealing after the irradiation will mobilize these gases, which will further create coalesced bubbles on the grain boundaries and cause significantly larger amounts of swelling that are no longer linearly related to the helium content (or fluence). This nonlinear swelling behavior is further accelerated by the reduction in the mechanical strength of the material [16]. Irradiation-temperature-dependent models based on the experimental data fits have also been derived and can be found, for example, in references [15] and [64]. However, these models are not applicable in the case of MURR reactor in which beryllium temperature doesn't reach $100^{\circ} \mathrm{C}$.

Since the swelling is physically caused by the production of transmutation gas, primarily He-4, only the data sets and the models that describe the swelling as a function of He-4 concentration were considered in the current study. While several of the data sets, as well as the models, describe swelling as a function of fluence, it is observed that such models do not provide consistent predictions, as energy spectra vary between various reactors.

Figure 3.37 presents the most relevant data and the models available in the literature from several research reactors, including ATR [8], [78], SM [12], [59], [70], [14], BR2 [15], and HFIR [10]. The ANFIBE software model [16] is based on the data from several reactors, including BR2 and ATR. The data were collected for various grades of beryllium produced domestically and internationally (by the Russian Federation). The data suggest that the irradiation-induced swelling is not a function of beryllium grade. The swelling measurements are based on sample dimensional or density change.

The following equation describes the linear fit to the gathered data:

$$
\frac{\Delta V}{V}(\%)=0.0001114 \cdot G
$$

where $\mathrm{G}$ is He-4 accumulation measured in appm.

Equation (3-5) is plotted with a solid black line in Figure 3.37. Additionally, a dashed black line representing the upper bound of the $95 \%$ confidence interval for the mean fit is included in the plot. The fit and the confidence intervals were evaluated. The current fit is very close to the fits produced by Billone (ATR) in [78] and Sannen (BR2) in [15], and the function implemented in ANFIBE software [16]. 


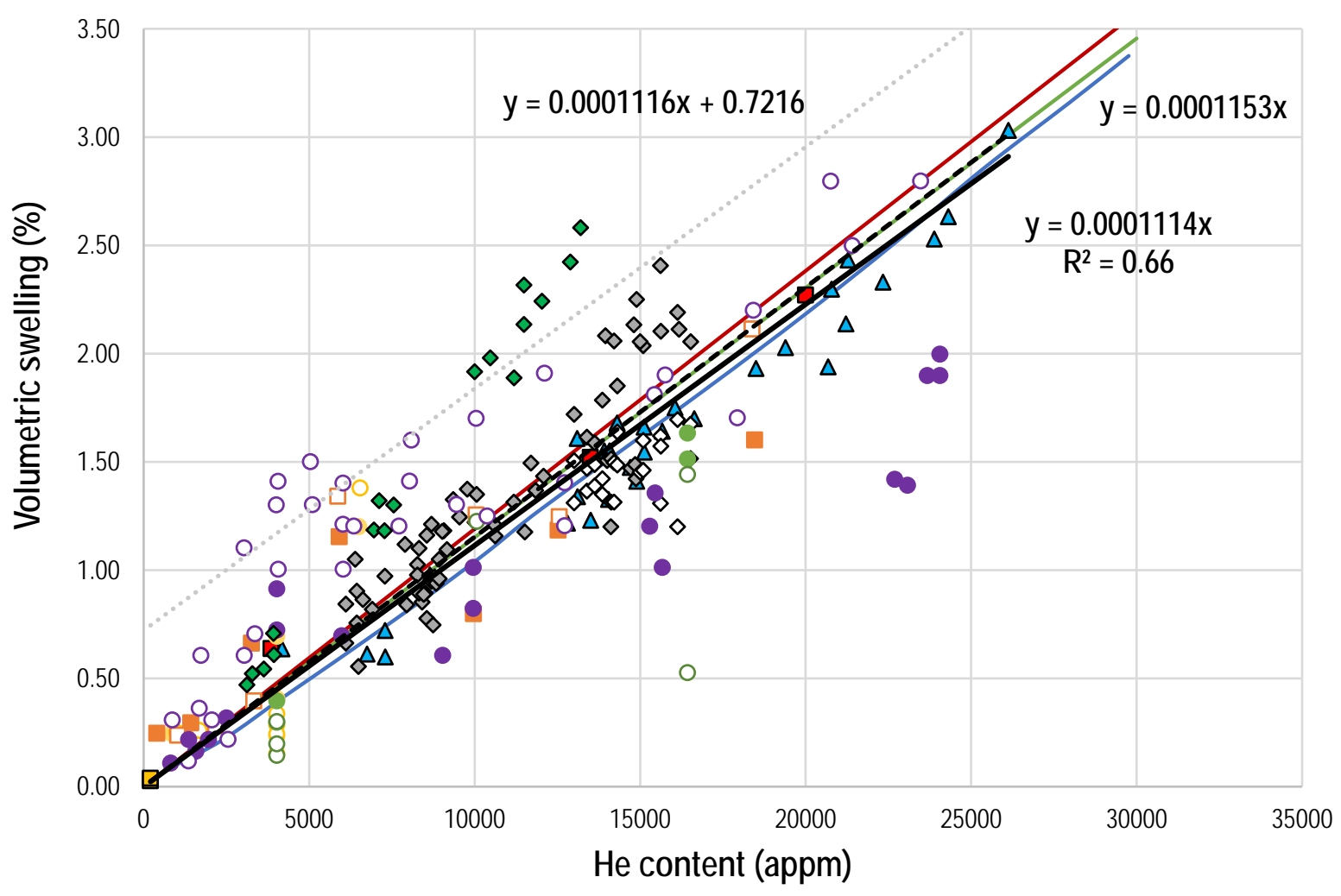

\begin{tabular}{|c|c|c|c|}
\hline & Billone (ATR) 1995 model & 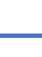 & ANFIBE model \\
\hline & Sannen (SCK) 1994 model & $\square$ & Sannen (BR2) 1994 density based \\
\hline$\Delta$ & Scaffidi-Argentina 1995 & $\diamond$ & Beeston (ATR) 1976 dimension based \\
\hline$\diamond$ & Beeston (ATR) 1976 density based & - & Chakin (SM) 2002 dimension based \\
\hline o & Chakin (SM) 2002 density based & - & Chakin (SM) 2004 dimension based \\
\hline 口 & Chakin (SM) 2004 density based & - & Chakin (SM) 2004b dimension based \\
\hline o & Chakin (SM) 2004b density based & $\bullet$ & Chakin (SM) 2011 dimension based \\
\hline 0 & Chakin (SM) 2011 density based & 口 & Snead (HFIR) 2004 \\
\hline$\diamond$ & Koonen (BR2) 1990 dimension based & - & Linear (all) \\
\hline & Linear (upper 95 indiv swelling) & - & inear (upper 95 mean swelling) \\
\hline
\end{tabular}

Figure 3.37. Volumetric swelling data for irradiated beryllium as a function of He-4 content

Figure 3.38 presents the volumetric swelling amount in the MURR reflector at 8 years of operation with HEU and LEU cores. On the basis of the model developed here (see equation (3-5)), the maximum swelling in the reflector after 8 years of operation will be about $0.45 \%$ and $0.50 \%$ with HEU and LEU cores, respectively. 


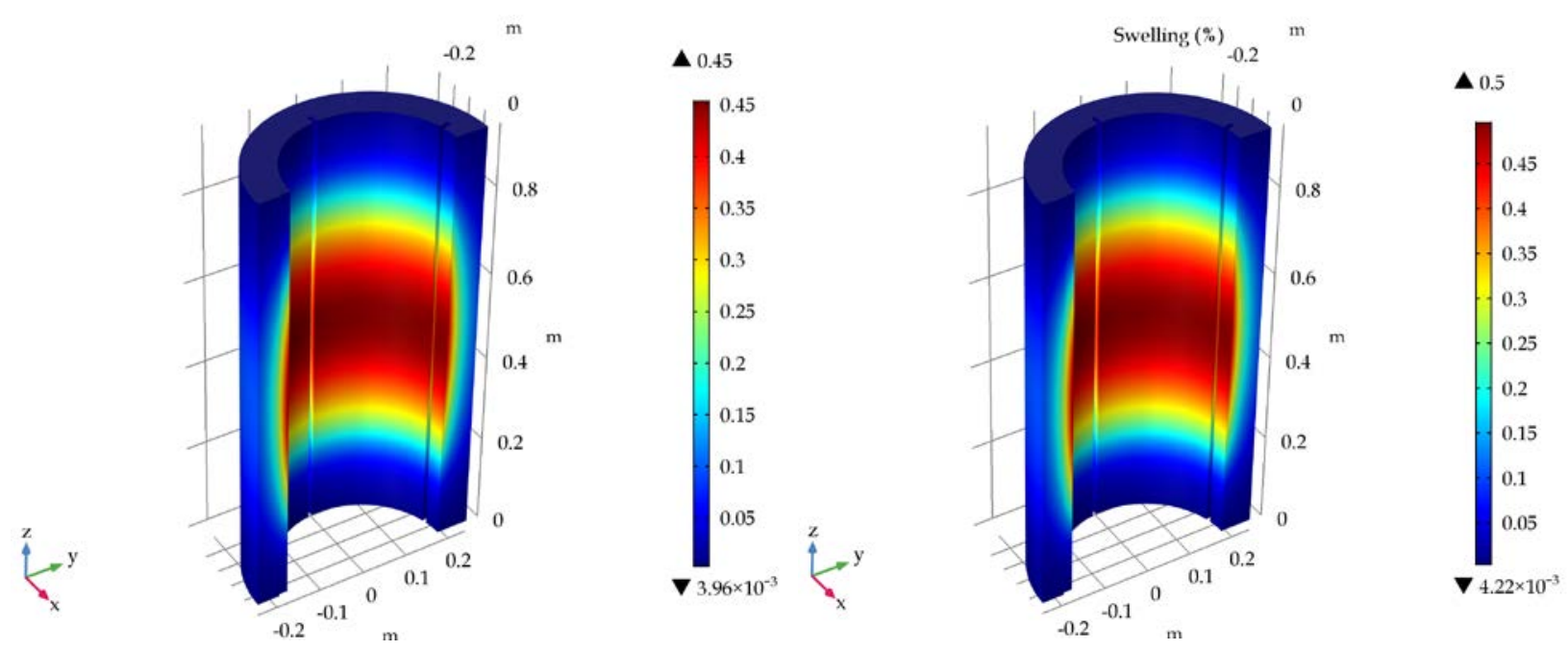

Figure 3.38. Swelling in MURR beryllium reflector after 8 years of operation with HEU (left) and LEU (right) cores

\subsection{Selection of Failure Criterion for Irradiated Beryllium}

The safety analysis acceptance criteria for non-power reactors in the US, including MURR, are established in NUREG-1537 [79], [80]. The acceptance criteria for reflectors include the following:

1. The design should allow for dimensional changes from radiation damage and thermal expansion to avoid malfunctions of the moderator or reflector.

2. The design should include experimental facilities that are an integral part of the reflector. If these facilities malfunction, the reflector components should neither damage other reactor core components nor prevent safe reactor shutdown.

3. The design should provide for removal and/or replacement of solid moderator or reflector components as systems, if required by operational considerations.

On the basis of these requirements, it is recognized that performance failure of a reflector as a component of the reactor may occur much sooner than mechanical failure of the material. For example, transmutation-gas-induced swelling of reflector components may cause dimensional changes large enough to block the gap between the reflector and any components that surround it. This blockage may cause problems with reflector cooling or safe removal of the irradiated reflector, or may impinge on operation of other components (e.g., the MURR reactivity control system). Excessive deformation of beryllium reflectors is a life-limiting factor at several reactors, including JMTR [9], SAFARI-1 in South Africa [81] [82], and HFR in Petten, The Netherlands [83]. However, in those cases where the impingement of the deformed reflector on surrounding structures is not considered an issue, reflector life will be limited by the increase of internal stresses due to heating and transmutation-induced swelling that will eventually lead to failure (fracture of material). At the same time, the reflector life will also be reduced by the degrading strength of the beryllium material due to irradiation. Historical inspections of beryllium reflectors at ATR [84] and BR2 [85], and the aforementioned failure at MURR in 1981, indicate that the reflectors at these locations can fail because of these internal stresses. 
Although fresh beryllium material (especially the new grades) behaves similar to most metals that exhibit yielding and hardening before a ductile (shear) failure, the failure mechanism of beryllium irradiated at low temperatures $\left(<100^{\circ} \mathrm{C}\right)$ is different. Under such conditions, beryllium loses its already low ductility and the material becomes brittle in both tension and compression with no yielding and hardening. The UTS of irradiated beryllium is significantly lower than the UCS, and the degradation of the two with irradiation progresses at different rates. Thus, any failure criterion which assumes ductile yield in tension and compression (like the von Mises criterion that is commonly used for ductile metals) cannot be applied to beryllium material irradiated at low temperatures. Moreover, the irradiation level in the reflector varies significantly in both the radial and axial dimensions (see Figure 2.1-Figure 2.3). As a result, irradiation-induced damage (in dpa) is greater at the inner surface and mid-height of the reflector than at the outer surface (Figure 2.7 and Figure 2.8). For that reason, failure limits must vary spatially in the model in accordance with the damage distribution, which is presented in Section 2.3.

With regard to the historical failure of the MURR reflector in 1981, it is assumed that failure of the reflector occurred at the point in time when at the mid-height of the reflector there was minimal ductility left in the material because of irradiation hardening. At the same time, portions of the reflector near the top and bottom had significantly less exposure to irradiation, and the material in these sections had substantial ductility left. Throughout this report, the major focus is on the failure mode similar to the 1981 failure located at the mid-height of the reflector. For that reason, only failure criteria for brittle materials are considered here.

As will be shown later in Section 4.6, there will be two most-stressed points of interest in the MURR beryllium reflector. Since both these points lie on the surface of the reflector, two principal stresses dominate, with the third one being close to zero. Therefore, two-dimensional (2D) failure criteria will be considered to evaluate the likelihood of failure. Additionally, of most importance for the life prediction of the MURR beryllium reflector is the definition of failure locus in the first quadrant of the 2D principal stress space where both principal stresses $\left(\sigma_{1}\right.$ and $\sigma_{2}$ ) are tensile (see Figure 3.39). It should be noted that positive principal stresses indicate tensile stresses, while negative values indicate compressive stresses. However, UCS is reported as a positive number.

The simplest failure theory for brittle materials is based on the maximum stress criterion, also known as the normal stress, Coulomb, or Rankine criterion [86]. According to this criterion, failure occurs when maximum principal stresses reach UTS or UCS. The failure can be defined in the 2D space of principal stresses $\sigma_{1}, \sigma_{2}$, as

$$
-U C S \geq \sigma_{2}, \sigma_{1} \geq U T S
$$

This criterion has been suggested for the design of beryllium reflectors since the 1960s [87]. It is currently used in the life prediction of reflector components at ATR [88]. The ATR life estimation procedure takes into account the degradation of the UTS of beryllium as a function of fluence (instead of dpa).

The failure locus of the maximum normal stress criterion in the 2D principal stress space is shown in Figure 3.39 by a dashed green line. The safe stress state is within the square defined by equation (3-6). To construct these boundaries, the UTS of fresh beryllium based on Table 3.1 was used. The UCS of fresh beryllium was not reported by Materion for S-200-FH [26]; therefore, a value of $1600 \mathrm{MPa}$ was assumed on the basis of the data from SM reactor tests, as shown in Figure 3.31. 


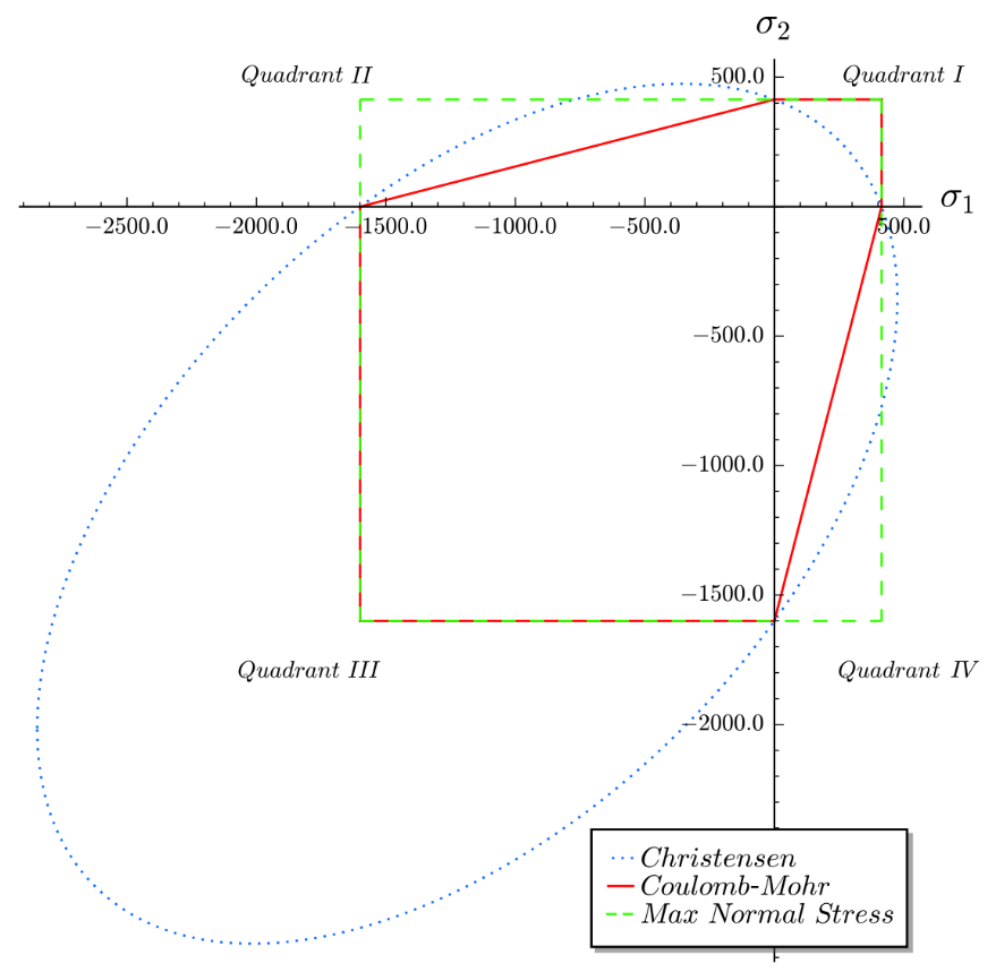

Figure 3.39. Comparison of failure criteria in 2D principal stress space

A more conservative criterion, known as the Coulomb-Mohr criterion, is based on the internal friction theory [86]. The theory predicts failure of material to occur when the combination of the maximum and minimum principal stress exceeds the limits listed in Table 3.4. The locus for the Coulomb-Mohr criterion is plotted in Figure 3.39 with a red line.

Table 3.4. Coulomb-Mohr criterion requirements

\begin{tabular}{|l|c|}
\hline \multicolumn{1}{|c|}{ Type of stresses } & \multicolumn{1}{c|}{ Failure condition } \\
\hline Both stresses in tension: $\sigma_{1}, \sigma_{2}>0$ & $\sigma_{1} \geq U T S$ and $\sigma_{2} \geq U T S$ \\
\hline Both stresses in compression: $\sigma_{1}, \sigma_{2}<0$ & $\sigma_{1} \leq-U C S$ and $\sigma_{2} \leq-U C S$ \\
\hline $\begin{array}{l}\sigma_{1} \text { in tension, } \sigma_{2} \text { in compression: } \sigma_{1}> \\
0, \sigma_{2}<0\end{array}$ & $\frac{\sigma_{1}}{U T S}+\frac{\sigma_{2}}{-U C S} \geq 1$ \\
\hline $\begin{array}{l}\sigma_{2} \text { in tension, } \sigma_{1} \text { in compression: } \sigma_{2}> \\
0, \sigma_{1}<0\end{array}$ & $\frac{\sigma_{2}}{U T S}+\frac{\sigma_{1}}{-U C S} \geq 1$ \\
\hline
\end{tabular}

As is the case for many brittle materials, there are no experimental data available to define the actual shape of the locus for irradiated beryllium in a biaxial stress field. However, for the materials for which such data are available, failure under biaxial tension is quite well predicted by the two simple criteria mentioned so far. They are equivalent to each other in the first and third quadrants of the principal stress plane (biaxial tension and biaxial compression). However, for some materials, the failure in quadrant I (biaxial tension) occurs sooner than these two criteria would predict [89], [90]. For that reason, Christensen proposed a failure criterion that assumes lower strength under combined biaxial tension. It is claimed, and supported by experimental results, to work well for some brittle and ductile materials [89], [90]. It is expected that the strength of irradiated beryllium in biaxial tension lies somewhere between the loci proposed by Christensen and Coulomb-Mohr. 
However, the exact shape of that locus can only be confirmed by the additional testing of irradiated beryllium samples.

Table 3.5 lists failure criteria based on Christensen's theory. A polynomial failure criterion is used for all types of materials (ductile and brittle). Its form in the 3D case and its simplified form in the 2D case are listed in the table. Additional failure criteria, together with the polynomial criterion, are used for brittle materials, for which $0 \leq \frac{U T S}{U C S} \leq \frac{1}{2}$, stipulating that none of the tensile stresses individually can be larger than UTS (as would be otherwise possible if only the polynomial criterion were used). Note that this additional criterion is equivalent to the limits set by the maximum normal stress criterion.

Table 3.5. Christensen criterion requirements [90] [91]

\begin{tabular}{|c|c|}
\hline Strength ratio & Failure condition \\
\hline For all materials: & in $3 D:\left(\frac{1}{U T S}-\frac{1}{U C S}\right)\left(\sigma_{1}+\sigma_{2}+\sigma_{3}\right)$ \\
$0 \leq \frac{U T S}{U C S} \leq 1$ & $\begin{array}{r}+\frac{1}{2 U T S \cdot U C S}\left[\left(\sigma_{1}-\sigma_{2}\right)^{2}+\left(\sigma_{2}-\sigma_{3}\right)^{2}+\left(\sigma_{3}-\sigma_{1}\right)^{2}\right] \geq 1 \\
\text { in } 2 D:\left(\frac{1}{U T S}-\frac{1}{U C S}\right)\left(\sigma_{1}+\sigma_{2}\right)+\frac{1}{U T S \cdot U C S}\left(\sigma_{1}^{2}-\sigma_{1} \sigma_{2}+\sigma_{2}^{2}\right) \geq 1\end{array}$ \\
\hline $\begin{array}{c}\text { Additionally for } \\
\text { brittle materials with } \\
\text { strength ratio: }\end{array}$ & $\sigma_{1} \geq U T S ; \sigma_{2} \geq U T S ; \sigma_{3} \geq U T S$ \\
$0 \leq \frac{U T S}{U C S} \leq \frac{1}{2}$ & \\
\hline
\end{tabular}

Figure 3.40 presents historical data on yield strength and ultimate failure limits tested in biaxial states for a historical beryllium grade produced by KBI (which was purchased by NGK Berylco in 1986 [92]). Together with these curves, lines representing failure limits according to the maximum normal stress criterion (dashed green) and the Christensen criterion (dotted blue) are plotted. These lines were produced using approximate values of about 440 MPa for UTS and about 540 MPa for UCS, corresponding to the values where the solid red line crosses the horizontal axis. The maximum normal stress criterion underpredicts the strength of that grade throughout the first quadrant (both stresses in tension), except in a small region where the corner of two green dashed lines crosses the experimental ultimate-failure line. The Christensen-criterion-based failure line in that quadrant is also entirely contained within the experimental ultimate-failure line. The Christensen failure line in the second quadrant follows precisely the experimental fit line. For this specific beryllium grade, UTS and UCS are close to each other. The Christensen criterion is a two-parameter criterion, in which UTS and UCS fully describe the shape of the failure locus. In cases where UCS is much larger than UTS (as is the case for S-200-FH), this criterion may greatly underestimate the strength of beryllium in biaxial tension ( $\sigma_{\mathrm{L}}$ and $\sigma_{\mathrm{T}}$ being the stresses in the longitudinal and transverse directions). The yellow dotted line represents a hypothetical grade which has the same UTS of $440 \mathrm{MPa}$ as the historical KBI beryllium but a much higher UCS of $1000 \mathrm{MPa}$. As can be noted, in the first quadrant, the yellow line is significantly below the red line. Thus, this criterion or other similar two-parameter criteria must be used with caution and need to be verified against experimental data. 


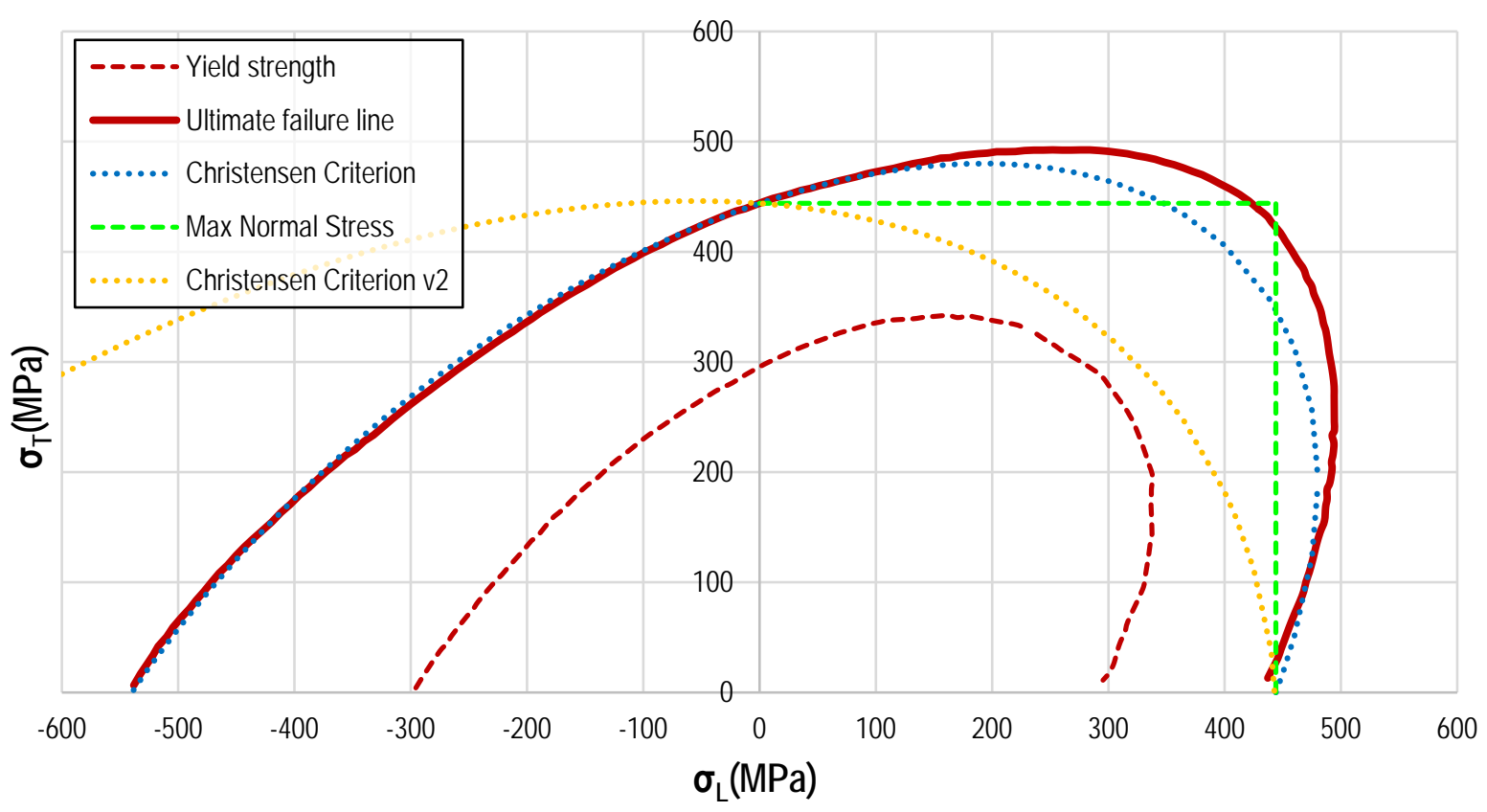

Figure 3.40. The effect of biaxial stress on yield and UTS of historic KBI beryllium as compared to various failure criteria

More complex criteria have been proposed for highly anisotropic rolled beryllium sheets, including the Tsai Wu criterion [93]. They contain more parameters describing the failure locus and, for that reason, require much more testing in complex states of stress. These criteria yield failure loci similar in shape to the one shown in Figure 3.40 (solid red line). Consideration should be given to these criteria when more data become available from multiaxial testing of beryllium.

The strength of any brittle material in tension is directly related to its surface condition and the presence of surface and subsurface cracks caused by the casting, machining, or finishing processes [29]. Any failure criterion based solely on the state of stress will only be valid if the surface condition of the examined solid body is equivalent to the condition of samples that were used to determine the ultimate strength of the beryllium material. This is usually not the case, and more modern failure theories, like those based on crack propagation phenomena, could be used to analyze the life of brittle materials, and of the MURR beryllium reflector in particular.

Fracture mechanics theories start with the assumption that cracks exist in mechanical parts even before they are put into service. The so-called linear elastic fracture mechanics approach focuses on the growth of a preexisting crack due to service loading. When a crack length exceeds the critical size, the crack propagates without further increase in loading, leading to failure of the component. Three modes of crack propagation exist [86]:

- Mode I - the most common mode, the opening crack propagation mode, due to tensile stress;

- Mode II - the sliding mode, due to in-plane shear; and

- $\quad$ Mode III - the tearing mode, arising from out-of-plane shear.

A so-called stress intensity factor for Mode I is defined as 


$$
K_{I}=\beta \sigma \sqrt{\pi a}
$$

with the units of $M P a \sqrt{m}$, where $\beta$ is a stress intensity modification factor, $\sigma$ is the applied stress in $\mathrm{MPa}$, and $a$ is the characteristic size of the crack in meters. The stress intensity factor is a function of stresses, geometry, size and shape of the crack, and the type of loading [86]. Tables for $\beta$ exist for most common geometries and locations of cracks. Figure 3.41 is a chart of the geometry and loading related to the MURR beryllium reflector (with some notable differences like more complex state of stress) recreated from [86]. For small cracks $<<$ the thickness of the reflector, the $\beta$ factor is about 1.1.
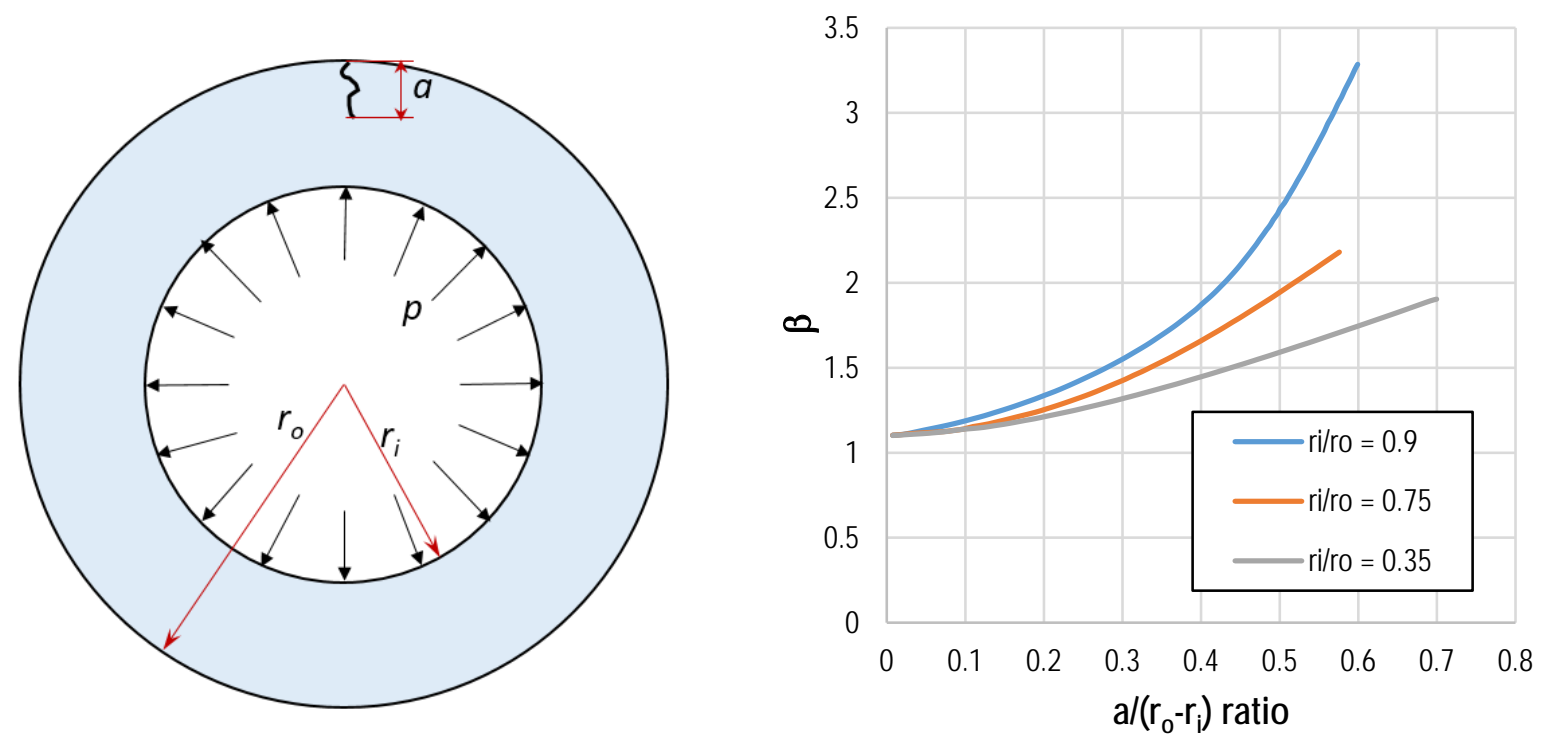

\section{Figure 3.41. Stress intensity modification factor for pressurized thick cylinders and cracks on the outer surface}

It is assumed that once $K_{I}$ reaches a critical value, called fracture toughness, for a specific material, crack propagation initiates, leading to a failure of the component. $K_{I C}$, just like tensile strength, is a material property that is dependent on temperature, loading rate, or state of stress. For irradiated beryllium material, $K_{I C}$ decreases with increasing fluence (or damage in terms of dpa).

As shown in Section 3.3.5, data on the fracture toughness of irradiated beryllium are scarce at low fluence and unavailable for the levels of fluence experienced by the beryllium reflector in the MURR reactor. Information on the size and location of preexisting cracks is also needed for life estimation based on these theories. The surface condition at the microscopic level of the MURR beryllium reflector is also largely unknown. Until these data become available, the material property $K_{I C}$, the size of the internal cracks, and the surface condition (crack size $a$ ) need to be assumed in the crackpropagation (Mode I) type of analysis. Thus, in our opinion, an approach based on crack propagation has limited use in the case of the MURR beryllium reflector. Despite the lack of complete information on the properties, an attempt is made to use limited available data to assess the relative strength of the analyzed reflectors with this approach. It must be noted that the crack propagation theories for the multi-dimensional state of stress are under constant development and there is no consensus on which one of them should generally be used [94], especially for materials as rarely used as beryllium. 
It is worth noting that the FE analysis in COMSOL does not rely on UTS and UCS. These are used to interpret the calculated state of stress or post-process the data only. Thus, the analysis presented in Section 4 will remain valid and when statistical evaluation of the strength properties of irradiated S200-FH beryllium becomes available, or a more appropriate failure criterion is proposed, the interpretation of the results (presented in Section 5) may be updated without the need to repeat the analysis. 


\section{Finite Element Model of MURR Beryllium Reflector}

\subsection{Geometry}

The geometry of the MURR reflector that will be used with LEU fuel will be identical to that of the current reflector used with HEU fuel. The current reflector does not have any penetrations for beamport holes. However, to estimate the relative life of the current reflector design following conversion of the fuel, the geometry of the reflector with beamport holes, which was the reflector design that failed in 1981, was also analyzed. It is expected that the features causing the largest stress concentrations leading to that failure were the spacer grooves on the inner face of the reflector and the edges around the beamport holes. Initially, all other geometrical features were ignored with the assumption that first, the most critical locations will be confirmed through the FE analysis using simplified models and coarse meshes.

The original MURR beryllium reflector cross section is shown in Figure 4.1, together with the details showing the geometry of edges around the beamport holes. Figure 4.2 shows the geometry of the reflector without beamport holes as built in a COMSOL software model to represent the current reflector design. Figure 4.3 shows the geometry of the first (and second) reflector design used at MURR with beamport holes as built in a COMSOL software model to represent the reflector that failed in 1981. All curvatures around the grooves and the beamport holes were modeled as closely as possible to the nominal dimensions found in the technical drawings of the reflectors.

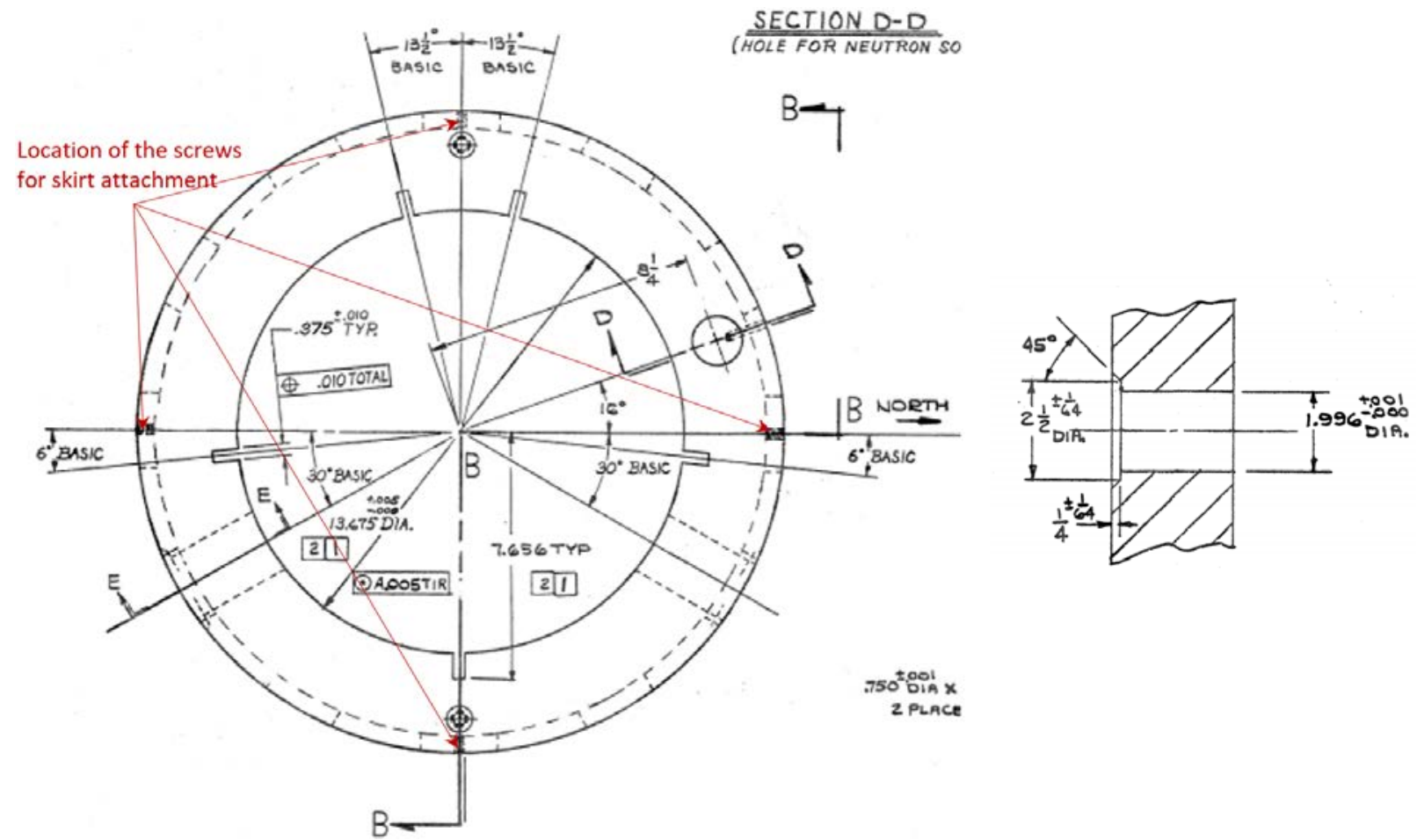

Figure 4.1. Cross section of MURR beryllium reflector with beamport holes 


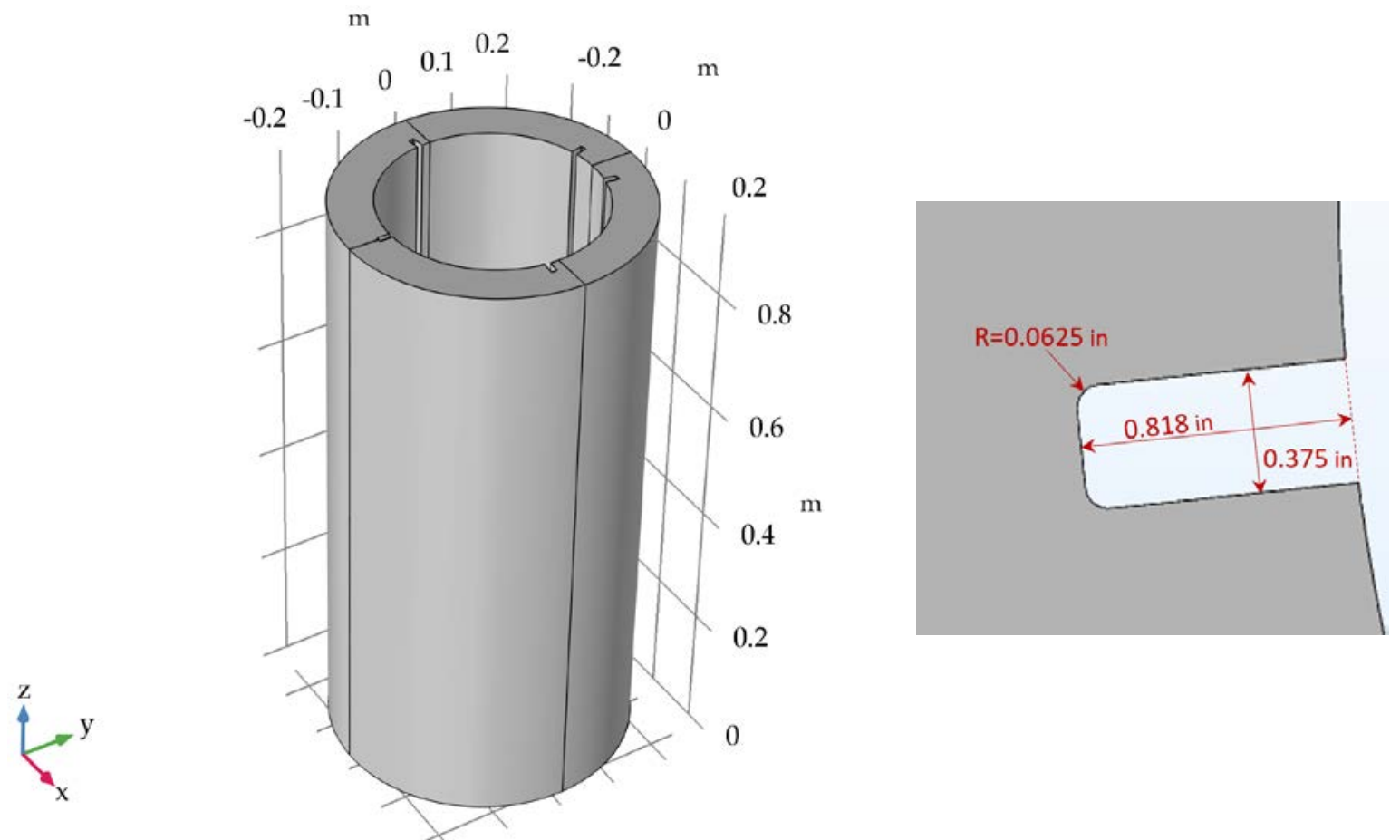

Figure 4.2. Geometry of the beryllium reflector model without beamport holes (left) and with a spacer groove (right)
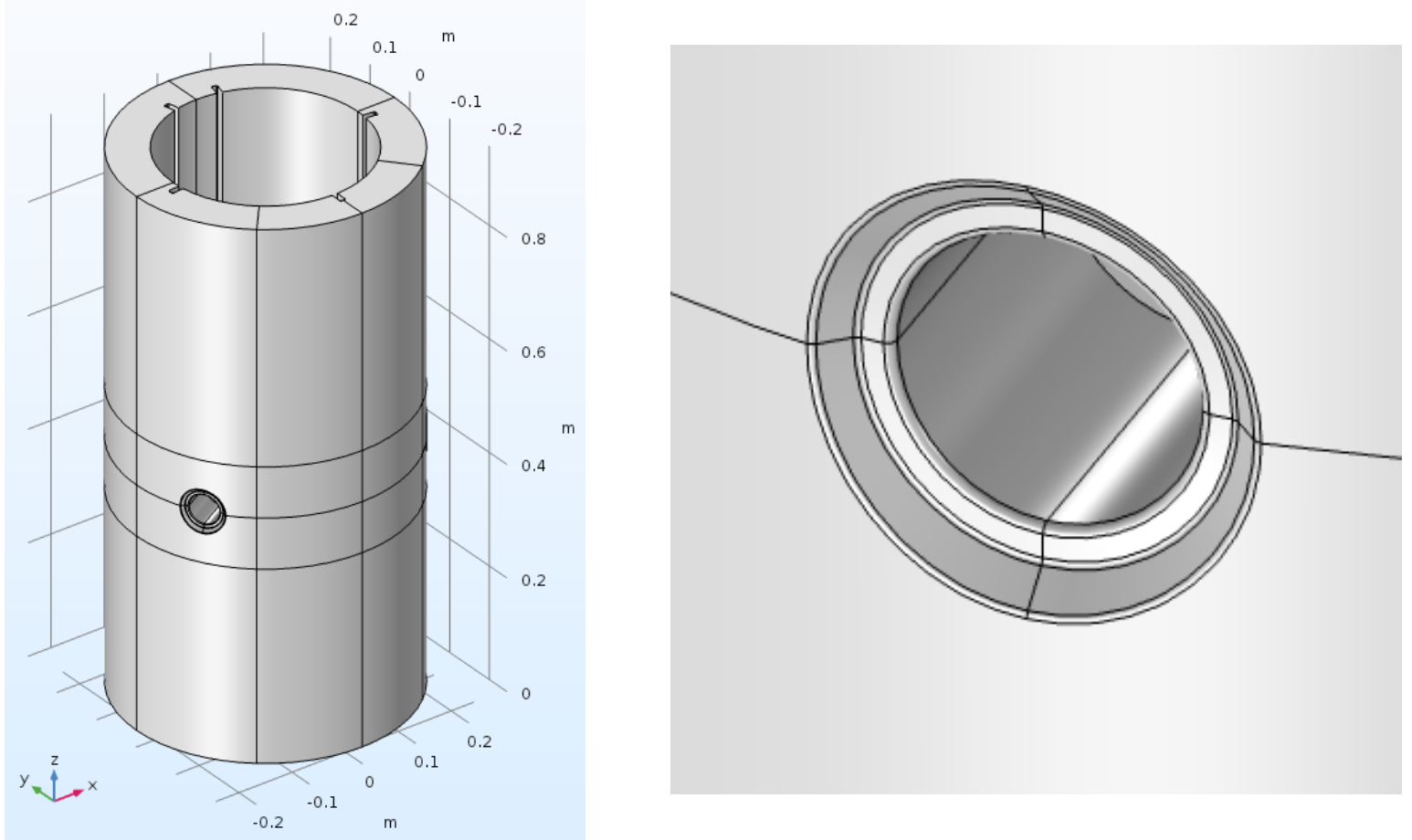

Figure 4.3. Geometry of MURR beryllium reflector with beamport hole (left), and detail of the beamport hole (right) 


\subsection{Finite Element Mesh}

Four aspects of mesh density were considered during the mesh development stage:

(1) The size of elements in a horizontal cross section of the reflector far from the grooves,

(2) The number of elements along the height of the reflector,

(3) The number of elements along the curvature of the spacer groove, and

(4) For the model with beamport holes, the density of the elements around the edge of the hole.

Figure 4.4 shows the model without the beamport hole, with the base mesh density.
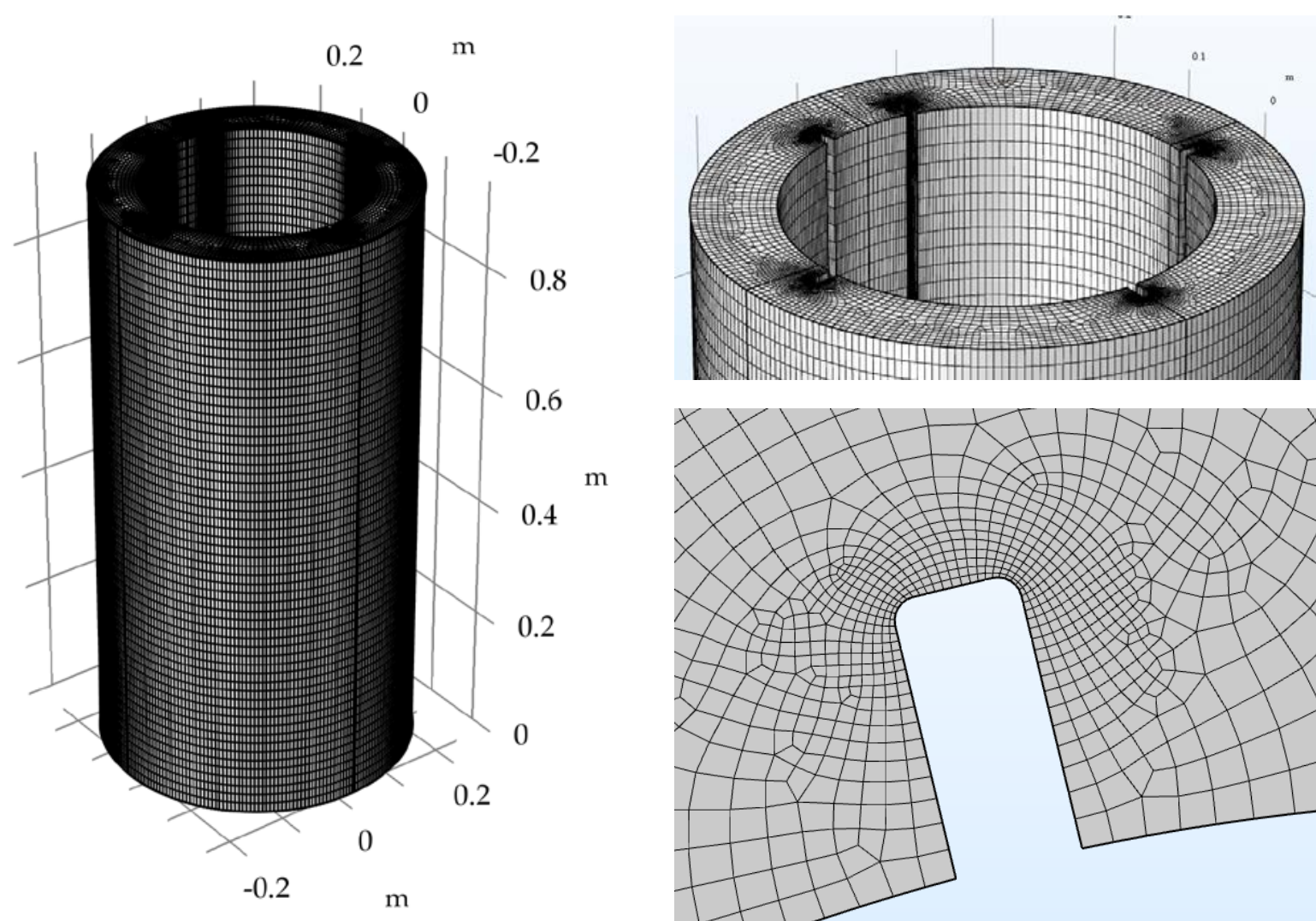

Figure 4.4. Mesh on the model without the beamport hole

\subsubsection{Size of Cells across the Thickness of the Reflector}

The mesh sensitivity study was performed on the model of the fourth and the subsequent reflectors (S-200-FH grade) without beamport holes, operating with a HEU core for 8 years. Four densities of mesh with quadratic elements in the cross section far from the grooves were studied to determine which is appropriate for the analysis. The reason to increase the density of the mesh through the reflector cross section is to properly capture the stress gradients and temperature distribution across the thickness of the reflector. Figure 4.5 shows the four mesh densities that were studied. The maximum $1^{\text {st }}$ principal stress on the outer surface of the reflector was chosen as a measure of the convergence of the results for most of the mesh parameters (except for the mesh density on the bottom of the groove, where the $3^{\text {rd }}$ principal stress was used for the convergence study). Table 4.1 lists the characteristic element size near the outer face of the reflector, the number of elements across the reflector thickness far from the groove, and the maximum $1^{\text {st }}$ principal stress predicted by each 
of these models. The differences in the predicted results are negligible. Consequently, any of these meshes is considered satisfactory for the convergence measure that was used. The mesh density with 12 quadratic elements across the thickness of the reflector (Figure 4.5(b)), noted as base mesh, was used for the final models. It gave smoother distributions of the temperatures and stresses across the thickness of the reflector than the coarse mesh. For the limiting cases quadratic elements have been used. For sensitivity studies, linear elements were used. For the linear as compared to the quadratic elements the savings in the computation time were roughly tenfold.

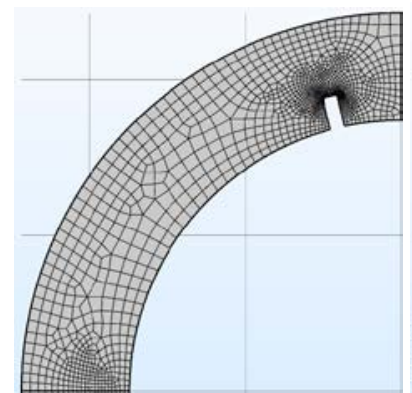

(a)

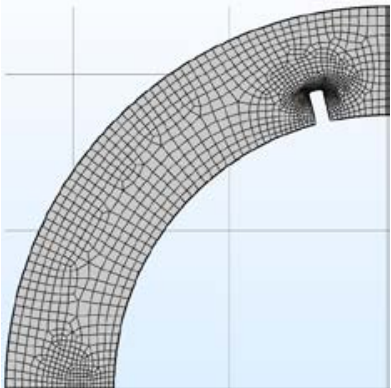

(b)

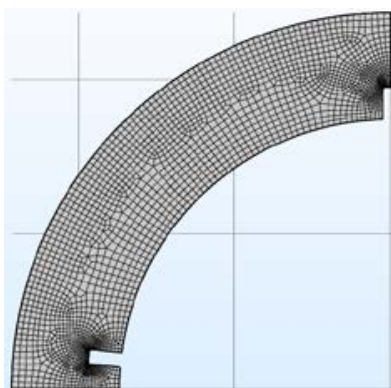

(c)

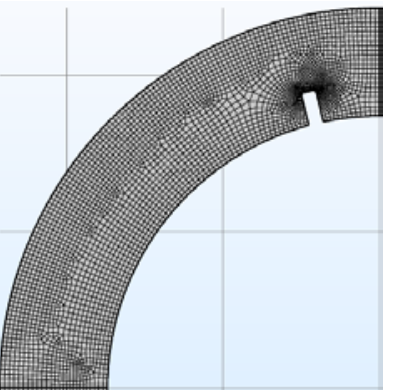

(d)

Figure 4.5. Various mesh densities in the cross section of the reflector model; from left to right: coarse (a), base (b), dense (c), and denser (d)

Table 4.1. Comparison of results for various mesh densities in the cross section of the reflector model

\begin{tabular}{|c|c|c|c|c|}
\hline & Coarse & Base & Dense & Denser \\
\hline $\begin{array}{c}\text { Element size on the outer } \\
\text { face (m) }\end{array}$ & 0.008 & 0.006 & 0.004 & 0.003 \\
\hline $\begin{array}{c}\text { Element count through } \\
\text { thickness away from grooves }\end{array}$ & 9 & 12 & 20 & 25 \\
\hline $\begin{array}{c}\text { Max 1st principal stress in } \\
\text { HEU model with linear } \\
\text { elements (MPa) }\end{array}$ & 250.1 & 250.2 & 250.8 & 251.0 \\
\hline $\begin{array}{c}\text { Max 1st principal stress in } \\
\text { HEU model with quadratic } \\
\text { elements (MPa) }\end{array}$ & 252.0 & 251.9 & 251.7 & 251.6 \\
\hline
\end{tabular}

\subsubsection{Size of Cells along the Height of the Model}

The gradients of heating and He-4 concentration are significantly smaller in the axial direction than they are in the radial direction (through the thickness of the reflector). For that reason, the model can have a smaller mesh density along the height. Table 4.2 compares the maximum $1^{\text {st }}$ principal stress (maximum tensile stress located on the outer surface of the reflector) in six reflector models. Linear and quadratic elements were used for three mesh densities in the axial direction. The models with quadratic elements have very smooth profiles of stresses through the thickness of the reflector and near stress concentration features. Additionally, the models with quadratic elements predict slightly larger maximum stresses than the models with linear elements, and thus are conservative for a study of reflector lifetime. However, the difference between the maximum stresses in the base models with linear and quadratic elements is less than 1\%. A final model with 60 quadratic elements along the height was used for smoother distributions of temperatures and stresses through the thickness, as mentioned above. Convergence of stresses was also confirmed for the maximum compressive stress on the bottom of the spacer grooves. 
Table 4.2. Comparison of results for various mesh densities in the cross section of the reflector model

\begin{tabular}{|c|c|c|c|}
\hline & Coarse & Base & Dense \\
\hline Element height (m) & 0.06 & 0.04 & 0.02 \\
\hline Element count in axial direction & 40 & 60 & 120 \\
\hline $\begin{array}{c}\text { Max 1st principal stress in LEU model } \\
\text { with linear elements (MPa) }\end{array}$ & 250.3 & 250.2 & 250.2 \\
\hline $\begin{array}{c}\text { Max 1st principal stress in LEU model } \\
\text { with quadratic elements (MPa) }\end{array}$ & 251.9 & 251.9 & 252.0 \\
\hline
\end{tabular}

\subsubsection{Number of Cells along the Groove Curvature}

The grooves are locations where stress concentration occurs. To capture this effect properly, the mesh density around the grooves was examined. Figure 4.6 shows models with mesh around the grooves with three, six, and nine elements along the groove fillet. Table 4.3 shows the minimum $3^{\text {rd }}$ principal stresses (or maximum compressive stress) around the groove in these test cases. The difference in the stresses between 6 and 9 elements along the groove fillet is $0.5 \%$ or less. In the final models, 6 quadratic elements along the groove fillet were used. The smallest element edge size in that model was about $0.0005 \mathrm{~m}(0.5 \mathrm{~mm})$.

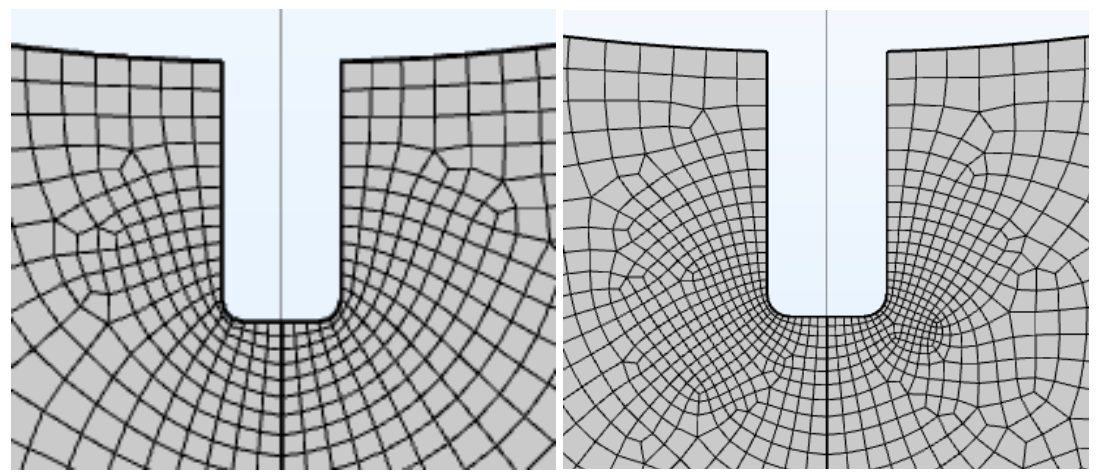

(a) (b)

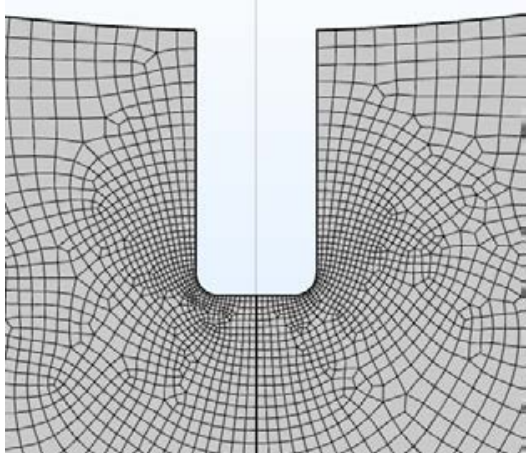

(c)

Figure 4.6. Mesh near the spacer groove. From left: coarse (a), base (b), dense (c)

Table 4.3. Comparison of results for various mesh densities in the cross section of the reflector model

\begin{tabular}{|c|c|c|c|}
\hline $\begin{array}{c}\text { Number of elements along the groove } \\
\text { corner fillet }\end{array}$ & $\mathbf{3}$ - coarse mesh & $\mathbf{6}$ - base mesh & $\mathbf{9}$ - dense mesh \\
\hline $\begin{array}{c}\text { Max 3 } \\
\text { with linear elements (MPa) }\end{array}$ & $-1,045$ & $-1,076$ & $-1,073$ \\
\hline $\begin{array}{c}\text { Max 3rd principal stress in LEU model } \\
\text { with quadratic elements (MPa) }\end{array}$ & $-1,022$ & $-1,079$ & $-1,076$ \\
\hline
\end{tabular}

\subsubsection{Size of Cells near the Beamport}

In order to predict realistic stress distributions around the beamport holes, their exact geometry needs to be modeled. The complex shape of the bevel could not be modeled with structured hexahedral elements. Free meshing with tetrahedral elements had to be used instead. First, the model 
was sliced with two horizontal planes and two vertical planes to isolate the region around both beamport holes (see Figure 4.7). The upper and lower portions were meshed with 25 layers of elements and the middle portion with 30 elements in the axial direction. Only the regions near the beamports were modeled with tetrahedral mesh. COMSOL has a predefined setting for the mesh densities when using free tetrahedral mesh in the model, which can be manually adjusted if needed. Four of these densities-fine, finer, extra fine, and extremely fine-were investigated, and the maximum $1^{\text {st }}$ principal stress (maximum tensile stress) on the beamport edge was tracked. The differences in these meshes are shown in Figure 4.8. Only the extremely fine mesh was able to capture the curvature of the entry to the beamport hole properly. Table 4.4 shows convergence of the results with increasing mesh density. Compared to the model with extremely fine mesh, the model with fine mesh predicted a maximum stress about $6.5 \%$ greater. The model with extra fine mesh predicted a maximum stress that is $1 \%$ larger than the extremely fine model. Although the results of the model with extra-fine mesh are considered adequate, the extremely fine mesh near the beamport holes with quadratic elements was used for the final models.

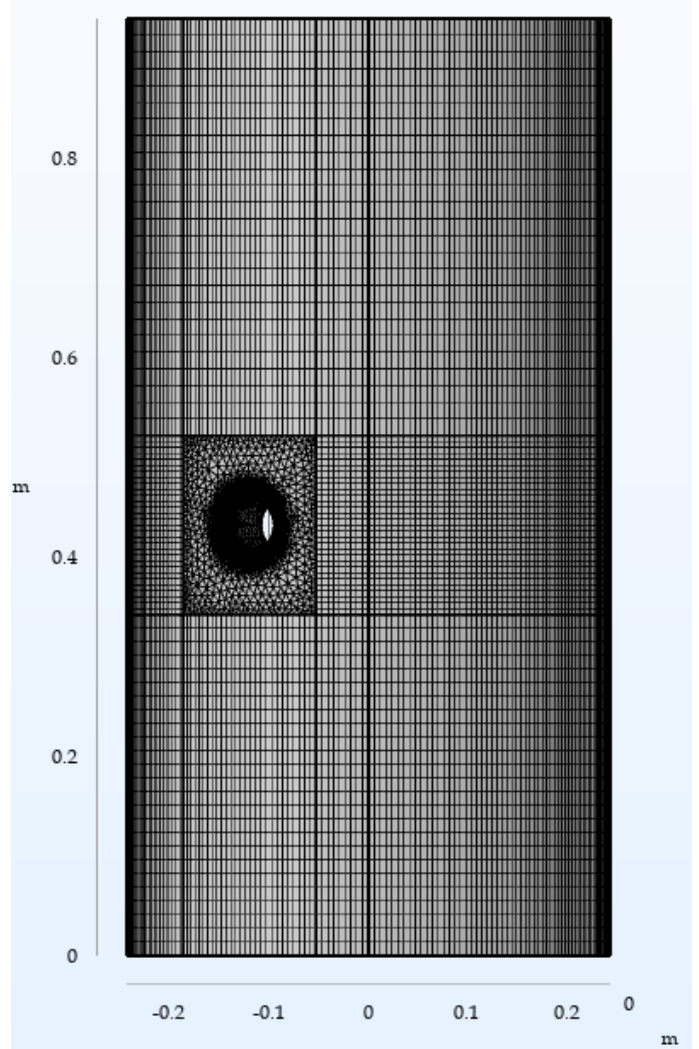

Figure 4.7. Mesh in the model with beamport holes

Table 4.4. Comparison of results for various mesh densities in the cross section of the reflector model

\begin{tabular}{|c|c|c|c|c|}
\hline & Fine & Finer & Extra Fine & $\begin{array}{c}\text { Extremely } \\
\text { fine }\end{array}$ \\
\hline Total element count in the model & 659,457 & 671,862 & 737,023 & $1,122,742$ \\
\hline $\begin{array}{c}\text { Max 1st principal stress in LEU } \\
\text { model (MPa) with quadratic } \\
\text { elements }\end{array}$ & 426 & 413 & 403 & 400 \\
\hline
\end{tabular}



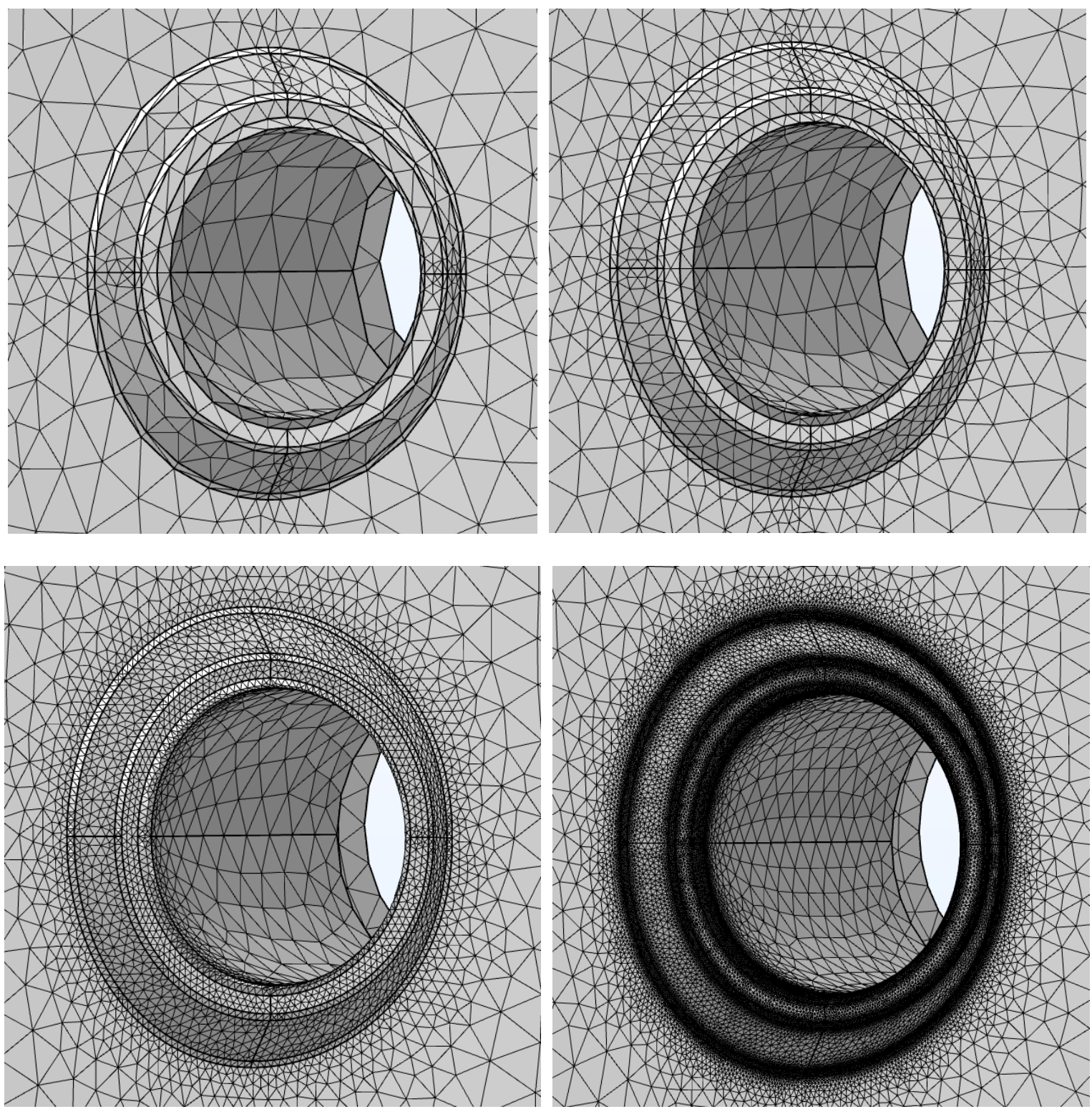

Figure 4.8. Various mesh densities around the beamport holes: (top) fine, finer, (bottom) extra fine, extremely fine

\subsection{Mechanical Boundary Conditions and Loadings}

Figure 4.9 shows a photo of the MURR beryllium reflector with the installed aluminum skirt around the base of the reflector and a schematic of the cross-section through the reflector and the skirt. The skirt is 0.5475 in. thick. A 1.75-in. rabbet is machined out of the outer surface of the beryllium material on the bottom and inserted into the skirt. Four holes are drilled through the skirt into the beryllium reflector. The reflector is then secured to the skirt with four screws that only have threads in the aluminum. A portion of the screw without threads extends into the beryllium and allows for independent expansion of the beryllium reflector and the skirt. This attachment creates complex mechanical boundary conditions that are not easy to replicate in a structural mechanics model. When the reflector expands as a result of heating and swelling, the contact surface between the reflector 
and aluminum skirt can slip. Additionally, the four screws/pins create small contact points, which give rise to localized stresses.

Figure 4.10 shows the geometry of the base of the machined beryllium reflector in the COMSOL model, which followed the technical drawings as closely as possible. Several FE models with this geometry were created in COMSOL and thermal-structural analysis was performed. During the development of the model, it was noted that one of the holes is drilled through one of the spacer grooves (see Figure 4.1 for reference). This feature caused problems with meshing. Models with this precise geometry had a significantly higher number of degrees of freedom (which was needed to resolve small curvatures), and some of them caused numerical problems with convergence. Various assumptions regarding the boundary conditions were made, resulting in a wide range for the predicted stresses at the bottom of the reflector. However, the assumptions regarding the boundary conditions on the bottom of the reflector did not influence the stresses in the mid-height of the reflector, where the most critical conditions influencing a life prediction are expected. In addition, the reflector failure in 1981 originated at the beamport edges, and the currently operating reflectors do not show any signs of failure near the skirt. For these reasons, it was decided that simplified boundary conditions on the bottom of the reflector would be pursued, and if possible, used in the final simulations. This choice resulted in a significant reduction of the number of degrees of freedom and made the numerical models more stable. It is advised that the locations of the holes drilled through the skirt and reflector at MURR be changed so that the holes are not aligned with the groove.
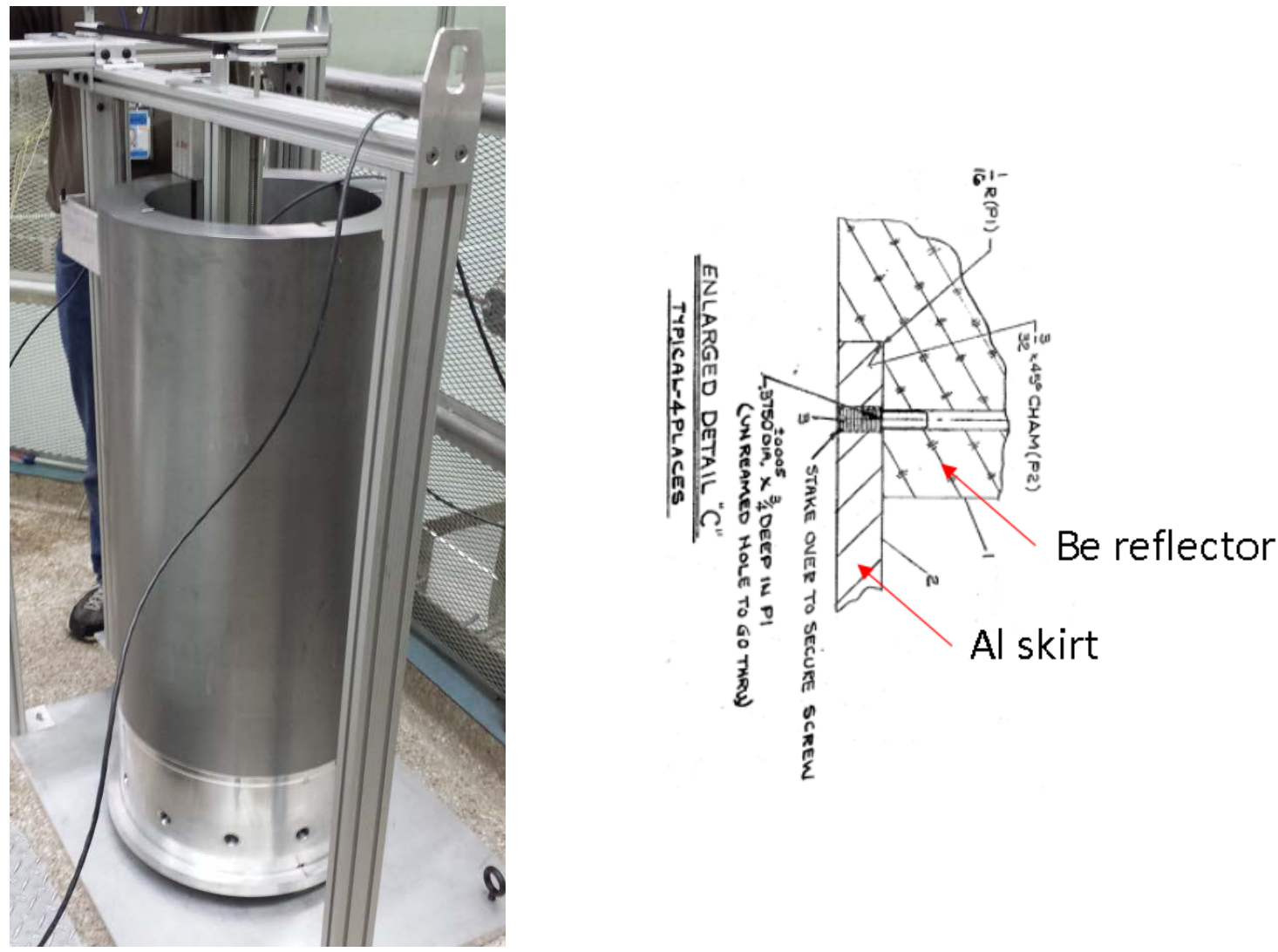

Figure 4.9. Beryllium reflector and aluminum skirt; (left) photo of MURR reflector with skirt, (right) detail sketch 

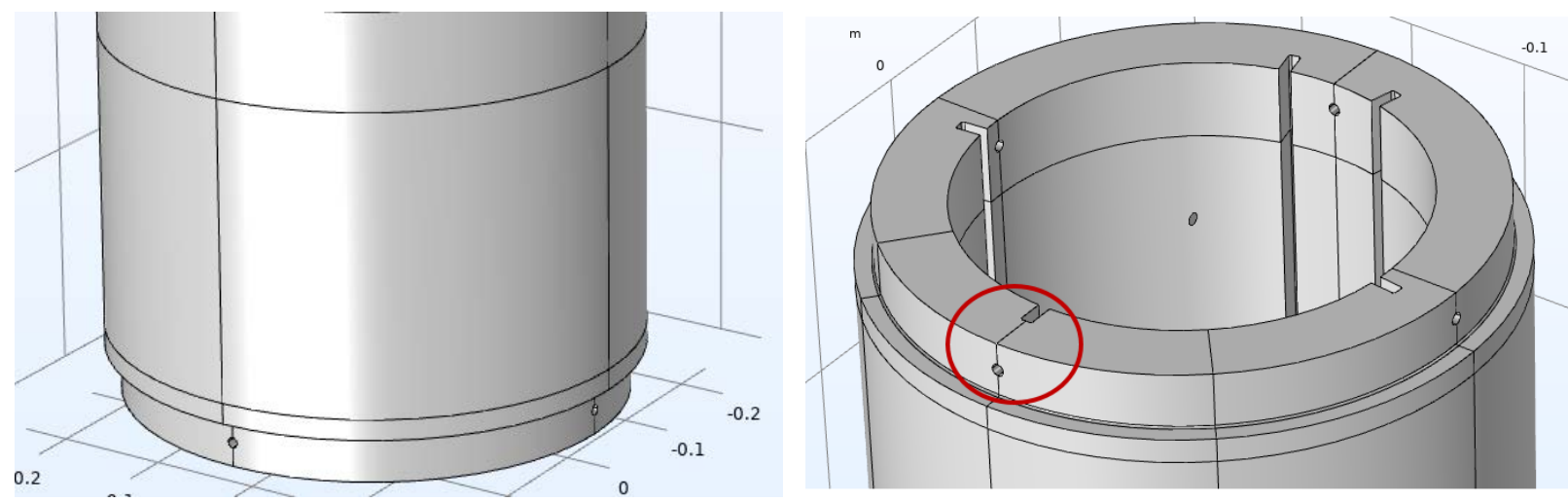

Figure 4.10. Geometry of beryllium reflector with exact geometry of the base

Figure 4.11 shows the areas where simplified boundary conditions were applied to the reflector. Symmetry boundary conditions were applied to the entire bottom surface of the reflector (constrained axial translations in $\mathrm{z}$ direction and rotations about $\mathrm{x}$ and $\mathrm{y}$ axes). On the inner edge of the bottom surface, spring boundary conditions were defined. All springs were modeled as acting in the radial direction from the center point on the bottom. Additionally, one point, as shown in the righthand panel, was fixed so that all rigid-body degrees of freedom for the reflector were constrained (a requirement for most structural solvers). The springs are not equivalent to the actual boundary conditions created by the contact with the aluminum skirt. However, they allow for expansion of the reflector without creating localized stresses on the bottom and without influencing the complexity of the model and increasing computation time. The stresses in that area are not representative, but these "soft" boundary conditions do not adversely influence the stresses predicted in the most critical areas of the reflector where transmutation-gas production and damage are the largest.
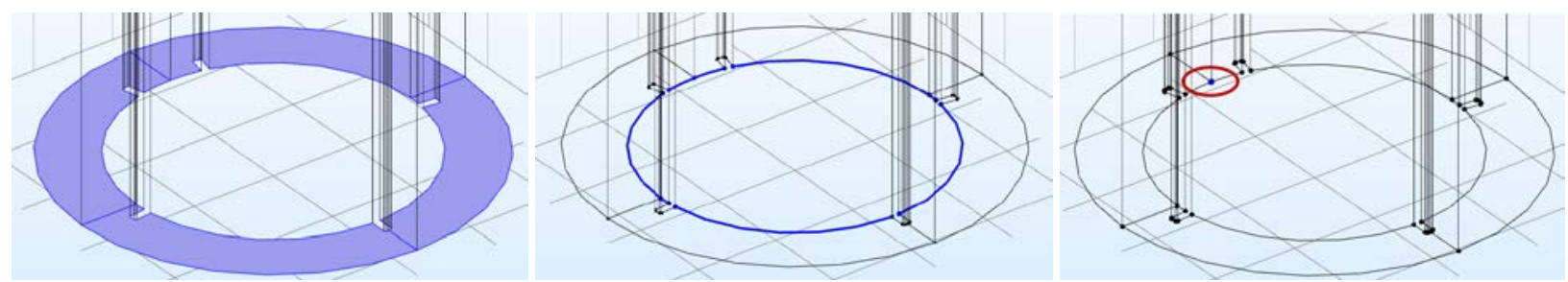

Figure 4.11. Mechanical boundary conditions on the bottom of the reflector

To confirm that the soft boundary conditions chosen for the model do not adversely affect the predicted maximum stress in the reflector, a most-limiting boundary condition case was simulated by constraining the entire bottom surface of the reflector, which creates a fully fixed boundary condition. Such constraints create more resistance to deformation than the actual method of attaching the reflector to the aluminum skirt. Maximum principal stresses were compared between the models with these two boundary conditions. Figure 4.12 shows the distribution of $1^{\text {st }}$ principal stresses in the model with soft boundary conditions (symmetry plane on the bottom with springs) and the model with fixed boundary conditions. The difference in the predicted maximum $1^{\text {st }}$ principal stress on the outer surface of the reflector for these models is only about $1 \%$, and is actually greater for the model with the soft boundary condition. Thus, the distribution of stresses in the mid-height appears to not be affected to any noticeable extent by the choice of the boundary conditions, which only have a localized effect near the base. 

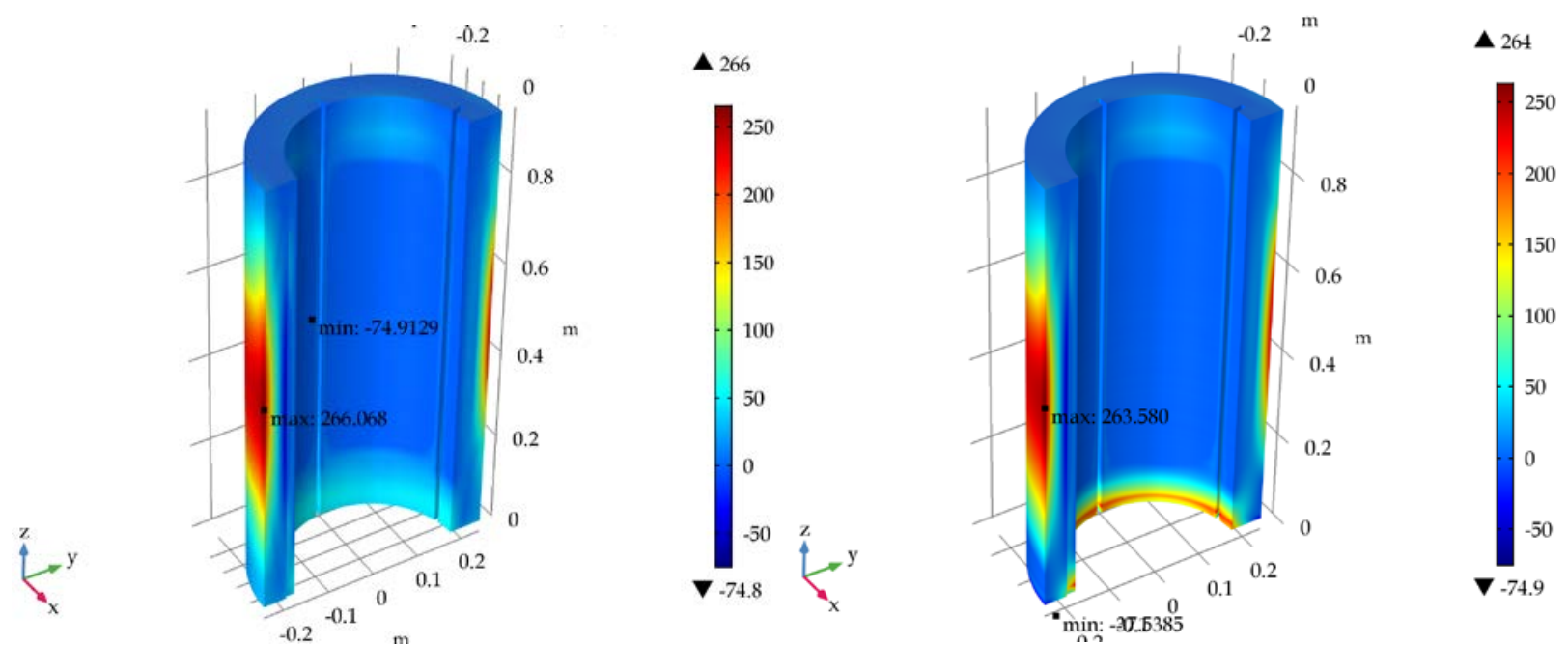

Figure 4.12. Comparison of $1^{\text {st }}$ principal stresses in models with soft (left) and fixed (right) boundary conditions at the bottom of the reflector

Figure 4.13 shows the distribution of the $1^{\text {st }}$ principal stresses in one of the test models, in which the most realistic geometry was used. Tetrahedral elements had to be used to model the fine details of the chamfer near the bottom of the reflector. At the mid-height, the stresses are not affected by the boundary conditions assumed on the bottom of the reflector. Near the base of the reflector, especially near the chamfer, extremely high stresses are predicted. However, since this area is not of major focus in the current study, for final models, the soft boundary conditions were chosen. If the lower portion of the reflector ever becomes of interest for failure analysis, the precise geometry of the reflector and accurate modeling of the contact between the reflector and the skirt will have to be considered.

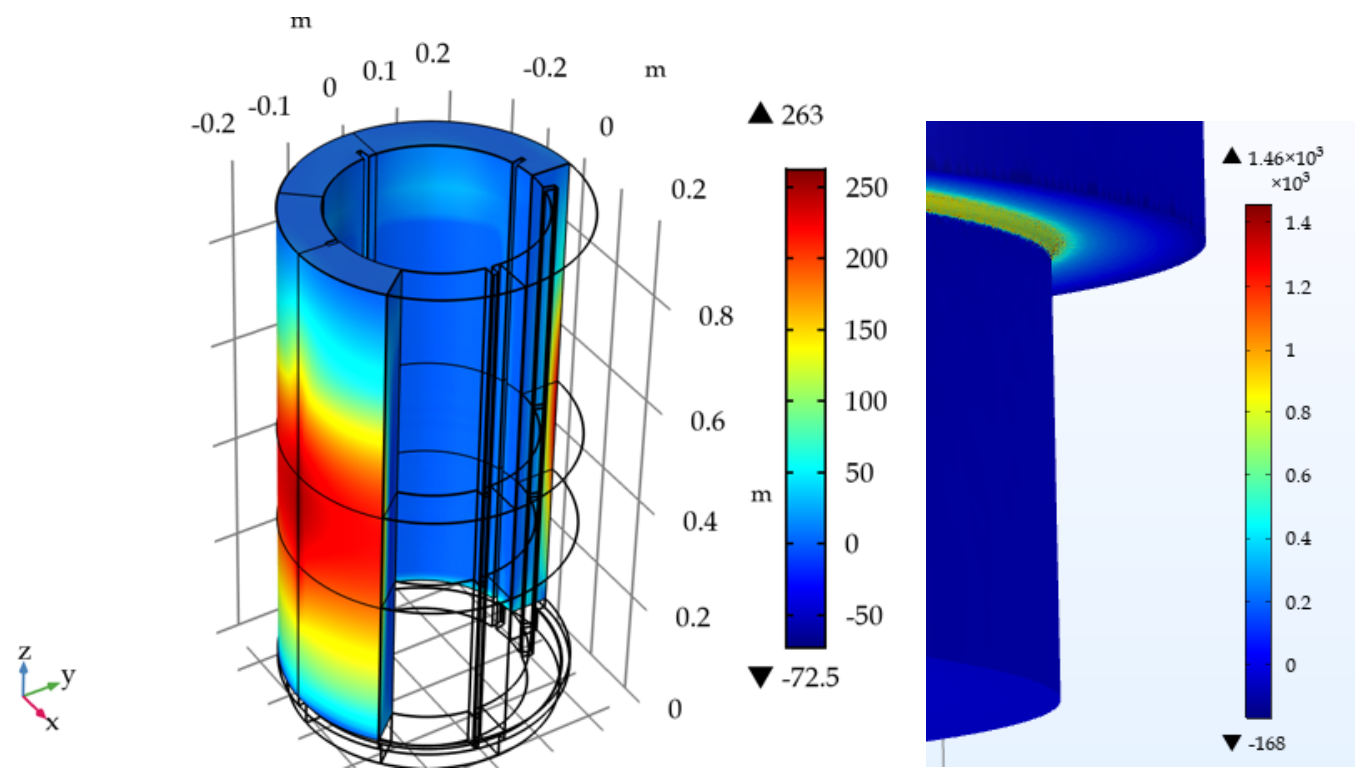

Figure 4.13. $1^{\text {st }}$ principal stresses in the model with exact geometry near the base of the reflector at the mid-height (left) and localized stresses near the base (right)

The strain tensor representing the total mechanical loadings in the analysis is built of two components: 


$$
\varepsilon^{t o t}=\varepsilon^{t h}+\varepsilon^{s w}+\varepsilon^{c r},
$$

where $\varepsilon^{t h}$ is the thermal strain tensor, $\varepsilon^{s w}$ is the swelling strain tensor, and $\varepsilon^{c r}$ is the irradiation creep. The thermal strain tensor components are defined as

$$
\varepsilon_{i j}^{t h}=\alpha_{i j}(T) \cdot \Delta T
$$

where $\alpha_{i j}(T)$ is the temperature-dependent coefficient of thermal expansion. In the current analysis, isotropic thermal expansion with a constant coefficient of thermal expansion was assumed, as per Table 3.1.

As noted above for equation (4-1), $\varepsilon^{s w}$ is the irradiation-induced swelling strain tensor. Again, isotropic behavior is assumed in the analysis and $\varepsilon^{s w}$ is defined as

$$
\varepsilon_{i j}^{s w}=\left\{\begin{array}{c}
\frac{\Delta V}{3 V} \quad \text { for } i=j \text { and } i, j=1,2,3 \\
0 \quad \text { for } i \neq j,
\end{array}\right.
$$

where $\frac{\Delta V}{V}$ is the swelling function fit defined in Section 3.4:

$$
\frac{\Delta V}{V}(\%)=0.0001126 \cdot H e 4 \_a c c u m u l a t i o n(a p p m)
$$

No suitable creep model was found in the literature. The irradiation creep causes reduction of stresses with time. Thus, omission of the creep model in this study leads to more conservative results.

\subsection{Thermal Boundary Conditions and Loadings}

For the thermal loading, the volumetric heating distributions from MCNP5 presented in Section 2.4 were applied as volumetric heat sources. For the inner vertical surface of the reflector, the heat transfer coefficient was estimated on the basis of the Dittus-Boelter correlation and approximate conditions in the coolant paths surrounding the reflector [95]. On the inner side of the beryllium reflector, there is a channel into which the control blades are raised and lowered to regulate the core reactivity during operation and for emergency core shutdown. The nominal water gap between the inner surface of the beryllium reflector and the outer surface of the reactor vessel is $0.0142 \mathrm{~m}(0.56$ in.), and the control blades are centered within this space.

During most of the reactor operating period, the control blades are positioned at about 23 in. withdrawn, which means that the bottom of the poison in the control blades has been raised 23 in. above its reference "full-in" position, or about 9 in. above the reactor core midplane. The control blades are $0.175 \mathrm{in}$. thick in the region of the active poison. Consequently, the spacing between the control blade and the beryllium reflector is $0.1925 \mathrm{in}$., and there is a corresponding 0.1925 -in.-wide space between the control blade and the outer surface of the pressure vessel. There is also a U-shaped edge component that is attached to the bottom of the control blade, which serves as a stiffener. This edge piece adds an additional 0.0375 in. to each side of the shim blade at the bottom (leading edge) 
of the blade. Consequently, the total thickness of the blade at this position is $0.250 \mathrm{in} .(0.0375+0.175$ +0.0375 ), which reduces the space between the beryllium and the leading edge of the control blades to 0.155 in. (0.1925-0.0375). However, this slightly reduced gap is not considered important for the cooling of the beryllium, since the space between the beryllium and the control blade along most of the axial dimension is the larger value of 0.1925 in. Additionally, from a thermal stress analysis perspective, worse conditions are expected for the case of a thicker channel, which leads to a lower heat transfer coefficient on the surface of the beryllium. Consequently, for further thermal-structural analysis, the full thickness of the channel, 0.56 in. $(1.422 \mathrm{~cm})$, was assumed.

The exact estimation of flow rates on both sides of the reflector is challenging without in-reactor measurements. Such measurements have not been performed. The total forced coolant flow rate in the pool is $1,200 \mathrm{gpm}$ by design. During design work for MURR, it was estimated that the flow rate between the pressure vessel and the reflector would be $215 \mathrm{gpm}$ [96], while on the outer side of the beryllium reflector the flow rate would be $100 \mathrm{gpm}$. The resulting coolant velocities are $4.5 \mathrm{ft} / \mathrm{s}$ and $4.25 \mathrm{ft} / \mathrm{s}$, respectively. The pool coolant temperature is $100^{\circ} \mathrm{F}\left(38^{\circ} \mathrm{C}\right)$ at the inlet and $106^{\circ} \mathrm{F}\left(41^{\circ} \mathrm{C}\right)$ at the outlet. Using these assumptions, a film coefficient of $4,260 \mathrm{~W} /\left(\mathrm{m}^{2}-\mathrm{K}\right)$ was estimated using the Dittus-Boelter correlation for the inner surface. For the outer surface, a film coefficient of 3,803 $\mathrm{W} /\left(\mathrm{m}^{2}-\mathrm{K}\right)$ was estimated. It should be noted that the Dittus-Boelter correlation based on the hydraulic diameter assumes the flow to be fully turbulent and fully developed, and uses a reference temperature equivalent to the local mixing-cup temperature.

In COMSOL, the heat-flux boundary condition is one for which $q_{0}=h \cdot\left(T_{\text {ext }}-T\right)$, where $T_{\text {ext }}$ is the temperature far away from the modeled domain; and the heat transfer coefficient, $h$, represents all the physics occurring between the boundary and a position that is "far away." The most common situation is that $h$ represents the effect of an exterior fluid cooling or heating the surface of a solid (convective cooling or heating) [97]. Since the difference between the inlet and the outlet temperatures is very small, $T_{\text {ext }}$ was assumed to be $39.5^{\circ} \mathrm{C}$, which represents an average temperature of the coolant around the reflector (the average of the pool inlet and outlet temperatures).

These values, together with the photon heat distributions presented in Section 2.4, define the boundary conditions and the loading in the thermal portion of the analysis. Table 4.5 compares the current estimations of film coefficients on the beryllium vertical surfaces to the values calculated in [95] during the reactor design stage. FE analysis was performed with these two sets of boundary conditions and the difference in the predicted maximum stresses ( 1 st principal stresses) was within $7 \%$, with the lower film coefficients leading to more conservative estimations of tensile stresses on the outer surface of the reflector. The same film coefficients are assumed for all analyzed cases (reflector with beamport hole operating with HEU core; reflectors without beamport holes operating with HEU and LEU cores). Thus, the impact of that assumption on all these cases is similar, and the main conclusions of this report are not affected by it.

Table 4.5. Film coefficient estimations on the vertical faces of the beryllium reflector

\begin{tabular}{|l|c|c|}
\hline & $\begin{array}{c}\text { Current } \\
\text { estimation }\end{array}$ & Internuclear Company estimation [95] \\
\hline Inner surface $\mathbf{W} /\left(\mathbf{m}^{\mathbf{2}} \mathbf{- K}\right)$ & 4,260 & $\begin{array}{c}6,757 \text { (below the bottom of the control rods) } \\
13571 \text { (above the bottom of the rods) }\end{array}$ \\
\hline Outer surface $\mathbf{W} /\left(\mathbf{m}^{\mathbf{2}} \mathbf{- K}\right)$ & 3,803 & 5,224 \\
\hline
\end{tabular}




\subsection{Matrix of Runs}

In the current analysis, two primary contributors to the overall state of stress-thermal and swellinginduced stresses-are taken into account. During the operation of the reactor, the thermal stresses are induced almost instantaneously but are reduced to zero when the reactor briefly shuts down as part of its normal weeklong cycle. The swelling-induced stresses increase slowly over time and are a function of transmutation-gas content. The following three states were considered:

a) Only thermal stresses are present in the beryllium reflector (initial state after turning on the reactor with a fresh beryllium reflector);

b) Only swelling stresses are present in the beryllium reflector (reactor is shut down at the end of its weekly cycle after 8 years of operation); and

c) Both thermal and swelling stresses are present in the beryllium reflector (reactor operating under normal conditions after 8 years of operation)

Note that in the initial state (case a), the thermal conductivity is constant and equal to the conductivity in the fresh (unirradiated) state (see Table 3.1). For the other two cases, the thermal conductivity distribution is based on the degradation fit as presented in Section 3.3.

These three states, combined with two geometries (with and without beamport holes) and two fuel element types (HEU and LEU), resulted in 9 analyzed base cases as listed in Table 4.6.

Table 4.6. Matrix of analyzed base cases

\begin{tabular}{|c|c|c|c|}
\hline & Heating only & Swelling only & Heating and swelling \\
\hline HEU with BPH (N-200-A) & $1 \mathrm{a}$ & $1 \mathrm{~b}$ & $1 \mathrm{c}$ \\
\hline HEU, no BPH (S-200-FH) & $2 \mathrm{a}$ & $2 \mathrm{~b}$ & $2 \mathrm{c}$ \\
\hline LEU, no BPH (S-200-FH) & $3 \mathrm{a}$ & $3 \mathrm{~b}$ & $3 \mathrm{c}$ \\
\hline
\end{tabular}

No transient analysis was performed for cooling-down and heating-up conditions. Simulating such conditions would require much more understanding about the strain rate-dependency of beryllium properties at various temperatures, which are not available in the literature. Since the temperature difference between the surface and the inside of the beryllium reflector is not very large $(\sim 30 \mathrm{~K})$, no significant additional stresses are expected. Also, as shown in Section 5.1, the current model predicts the swelling-induced stresses to be responsible for about $80 \%$ of overall stresses (superposition of effects is not possible because of nonlinear properties, so this estimate is very rough). Thus, uncertainties related to the thermal stresses have less effect on the overall state of stresses.

To predict the life of reflectors, the simulations had to be performed for irradiation times longer than the current replacement schedule of 8 years. For the case of reflectors without beamport holes with HEU and LEU cores, additional simulations have been performed for 10 and 12 years of operation. These simulations resulted in four additional cases. Subsequently, all the simulations have been repeated with model uncertainties in order to achieve the most conservative results. The uncertainties considered are listed in Table 4.7. 
Table 4.7. List of uncertainties in the finite element models of the reflectors

\begin{tabular}{|c|c|}
\hline Source of uncertainty & Input to the model \\
\hline $\begin{array}{l}\text { Neutronics calculation uncertainty (methods, } \\
\text { modeling, and data) }+/-10 \%\end{array}$ & $\begin{array}{l}\text { Heating, fluence, He- } 4 \text { concentration, damage (dpa) } \\
\text { scaled by } 1.0667 \text { (for } 2 \sigma \text { ) }\end{array}$ \\
\hline $\begin{array}{l}\text { Axisymmetric neutronics model vs. localized data: } \\
\text { - } 9 \% \text { higher He- } 4 \text { concentration at the bottom } \\
\text { of the grooves, no direct impact on the outer } \\
\text { surface of the reflector; } \\
\text { - } 6 \% \text { damage higher on the outer surface, } \\
\text { behind the grooves }\end{array}$ & Damage scaled by 1.06 \\
\hline $\begin{array}{l}\text { Swelling model: } 95 \% \text { upper confidence interval } \\
\text { bound of mean fit }\end{array}$ & Scaling factor for the swelling fit: 1.036 \\
\hline $\begin{array}{l}\text { Material properties data: } \\
\text { - Thermal conductivity fit } \\
\text { - Strength degradation }\end{array}$ & $\begin{array}{l}95 \% \text { lower confidence interval bound of mean fit to } \\
\text { the thermal conductivity degradation data } \\
95 \% \text { lower confidence interval bound of mean fit to } \\
\text { the ultimate tensile strength degradation data }\end{array}$ \\
\hline
\end{tabular}

\subsection{Results}

\subsubsection{Stresses in Beryllium (N-200-A) Reflectors with HEU Core and Two Beamport Holes}

The results of simulations revealed that the most critical case occurs for the "c case" from Table 4.6, representing the normal operation conditions when both thermal and swelling stresses are present in the reflector. The results presented in the following section correspond to this case.

Figure 4.14 presents the temperature distribution in the reflector with beamport holes after 8 years of operation with the HEU core. The peak temperature is found to be around $366 \mathrm{~K}\left(93^{\circ} \mathrm{C}\right)$. This finding confirms that the properties used in FE modeling should be limited to those reported for irradiation and testing temperatures around $100^{\circ} \mathrm{C}$.
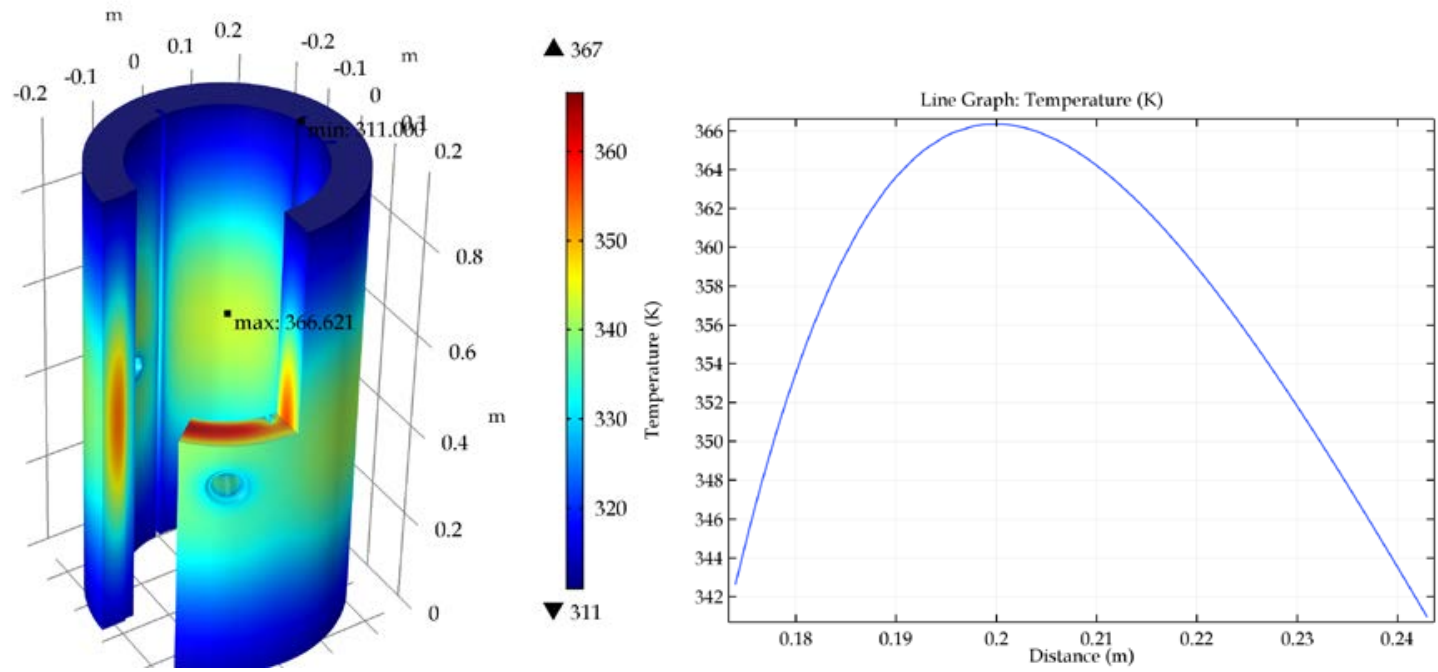

Figure 4.14. Temperature distribution in the FE model (left) and the distribution of temperature through the thickness of the reflector in the hottest location along the $y$-axis (right), in beryllium reflector with beamport hole after 8 years of operation with HEU core 
Figure 4.15 shows the distribution of the $1^{\text {st }}$ and $2^{\text {nd }}$ principal stress in the vicinity of the beamport hole in the beryllium reflector operating for 8 years with the HEU core. The principal directions of these stresses are shown as well with red arrows indicating the direction of 1 st principal stress and the green arrows indicating the directions of the $2^{\text {nd }}$ principal stress. The second principal stress was about $5 \mathrm{MPa}$ and the third one was even closer to zero. Thus, they are not significant contributors to a three-dimensional (3D) state of stress on the surface, near the beamport hole.

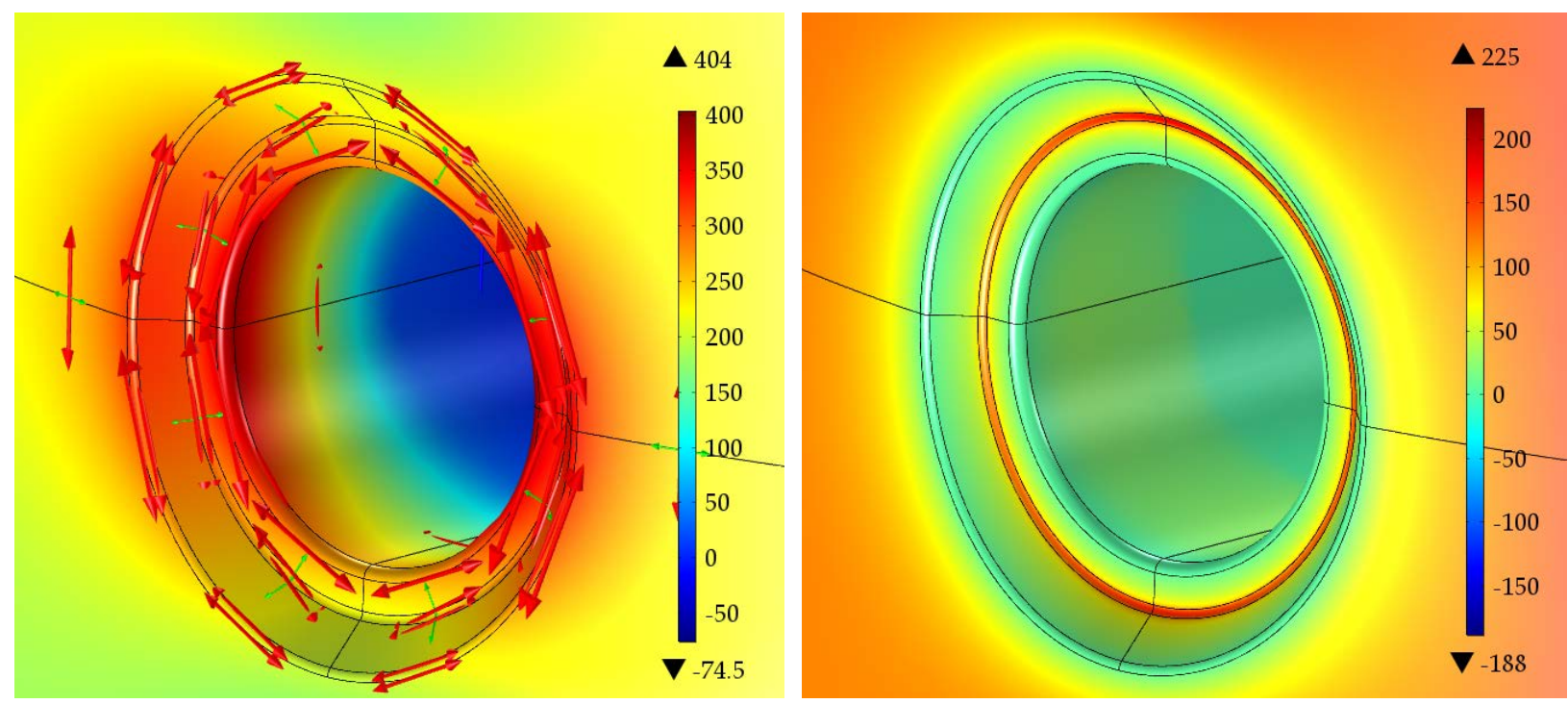

Figure 4.15. $1^{\text {st }}$ (left) and $2^{\text {nd }}$ (right) principal stresses in the reflector at the location of the beamport hole after 8 years of operation with HEU core

As explained in Section 3.5, the most critical stresses for brittle materials are usually the tensile stresses, since they lead to crack propagation. For the model with the beamport hole, the largest tensile stresses were found to be located on the outer edges of the beamport hole (see Figure 4.15). The $1^{\text {st }}$ principal stress (largest tensile stress) at this location was predicted to be $404 \mathrm{MPa}$ for the combined swelling and heating loads. That value is greater than the UTS of fresh beryllium grades available from Brush Beryllium in the 1960s, when the first reflector was manufactured and installed. The assumed minimum UTS of beryllium N-200-A was $276 \mathrm{MPa}$ (see Table 3.1). Thus, even without taking into account any strength degradation due to irradiation, the calculated stresses near the beamport hole were greater than the assumed minimum strength in tension, although the actual UTS of the installed beryllium block could have been greater than this assumed value. However, there exists a high probability that the tensile stresses after 8-year-equivalent operation of the beryllium reflector with beamport holes were higher than the actual strength of the material. The second reflector, which was installed in 1981, was made of the same material and had beamport holes machined in it. It operated for about $26,000 \mathrm{MWd}$, a period only $5 \%$ shorter than the operation period of the first reflector, which failed after 27,100 MWd. The authors of this report believe that although the second reflector was at a stress state very close to its material limits, it didn't fail because it may have had a better fabrication process and/or surface quality than the first one.

Figure 4.16 presents the ratio of $1^{\text {st }}$ principal stress to the UTS of beryllium N-200-A after 8 years of service at MURR. The maximum value on the scale was capped at 1.0 to highlight the zones where the degraded UTS was reached. The maximum value of that ratio was 1.61 near the entrance to the beamport hole. Figure 4.16 also shows that the areas on the outer surface of the reflector behind the spacer grooves were also experiencing stresses higher than the degraded UTS (very close to the UTS of fresh beryllium N-200-A). This indicates that in 1981, there could have potentially been several 
locations around the reflector at the mid-height where the tension was very close to or above the UTS, leading to the observed failure.

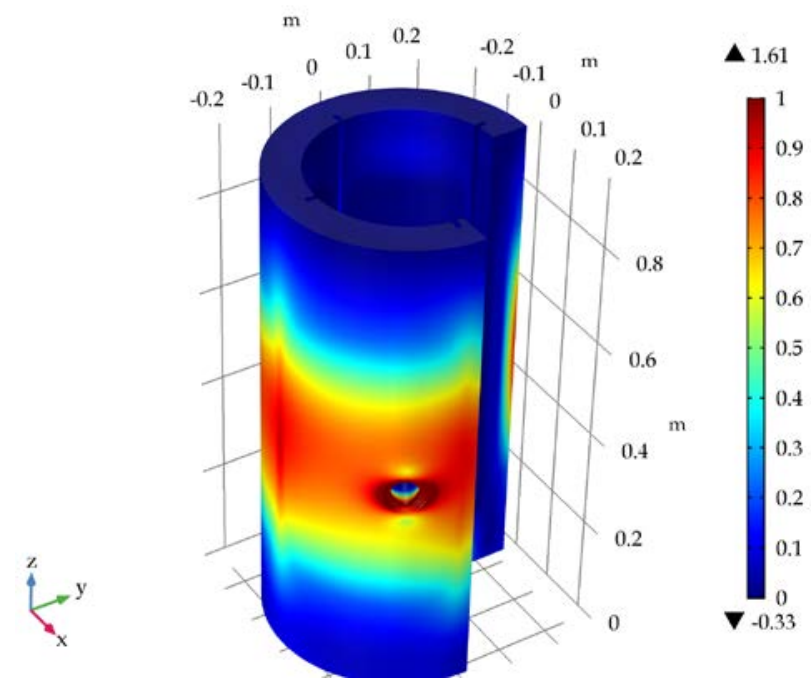

\section{Figure 4.16. Ratio of $1^{\text {st }}$ principal stress to local UTS in beryllium reflector with beamport} hole after 8 years of operation with HEU core

Figure 4.17 plots the $1^{\text {st }}$ and $2^{\text {nd }}$ principal stress in the reflector with the beamport hole and HEU core at 8 years of operation. The principal directions of these stresses are shown in Figure 4.18 with red arrows indicating the direction of $1^{\text {st }}$ principal stress and the green arrows indicating the directions of the $2^{\text {nd }}$ principal stress. In the areas near the mid-height of the reflector and behind the spacer grooves, not only the $1^{\text {st }}$ principal stress but also the $2^{\text {nd }}$ principal stress is predicted to approach values close to UTS. The predicted maximum $1^{\text {st }}$ principal stress in this area was $238 \mathrm{MPa}$ and the second principal stress was predicted to be $225 \mathrm{MPa}$. The direction of the $1^{\text {st }}$ principal stress is nearly vertical behind the grooves, while the direction of the $2^{\text {nd }}$ principal stress is nearly horizontal in that area. These are the preferred directions of crack propagation, with vertically oriented stresses causing horizontal cracks to open and vice versa. The $3^{\text {rd }}$ principal stress was close to zero, as shown in Figure 4.19. Because the $1^{\text {st }}$ and $2^{\text {nd }}$ principal stresses are both tensile and both of them are reasonably close to the UTS, a combined state of stress should be considered for these locations. Such a state of stress is more dangerous for a brittle material than the case where only one principal stress is dominant in tension and two others are close to zero or compressive. It is not clear which failure criterion should be used to analyze stresses at these locations. This question will be discussed in more detail in Section 5, together with the results from the two other reflector models (HEU and LEU cores and reflector with no beamport hole).

The lack of compressive stresses on the outer surface of the reflector is to be expected. The internal swelling and heating cause the reflector to expand and generate only tensile stresses on the outer surface of the reflector. Moreover, the He-4 production and heating are largest on the inner side of the reflector. For this reason, the stresses on the inner surface of the reflector are primarily compressive. By far, the largest compressive stresses ( $3^{\text {rd }}$ principal stress) occur on the bottom of the spacer grooves, around the mid-height. These stresses are highly localized because of the small geometrical features of the grooves, and reach -1,018 MPa for the reflector model with beamport holes. The $2^{\text {nd }}$ principal stress at that location is $-65 \mathrm{MPa}$. The $1^{\text {st }}$ principal stress is $-14 \mathrm{MPa}$. The principal directions with the dominant direction of $3^{\text {rd }}$ principal stresses are shown in Figure 4.20. Further interpretation 
of these stresses in comparison to other analyzed cases, namely, HEU core and reflector without the beamport hole as well as the reflector with the LEU core, will be presented in Section 5.
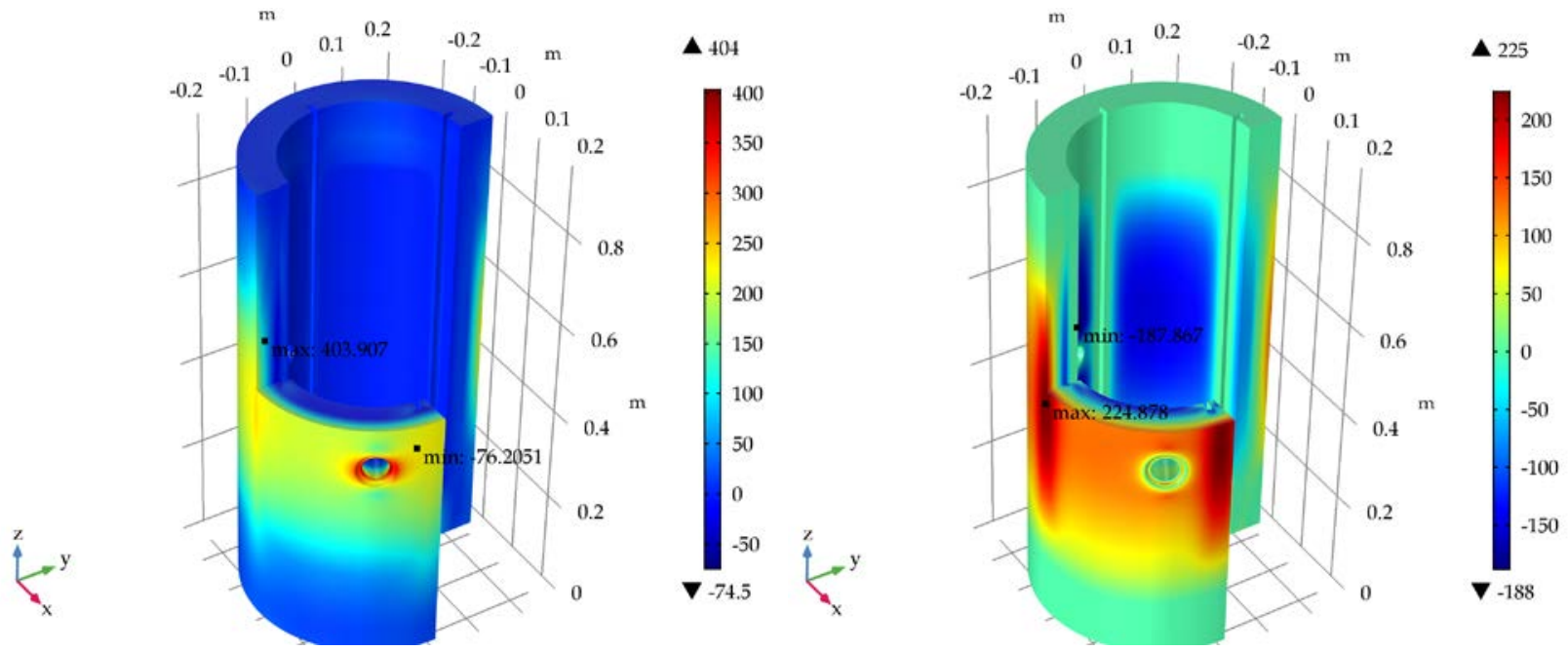

Figure 4.17. $1^{\text {st }}$ (left) and $2^{\text {nd }}$ (right) principal stress distribution in beryllium reflector with beamport hole after 8 years of operation with HEU core

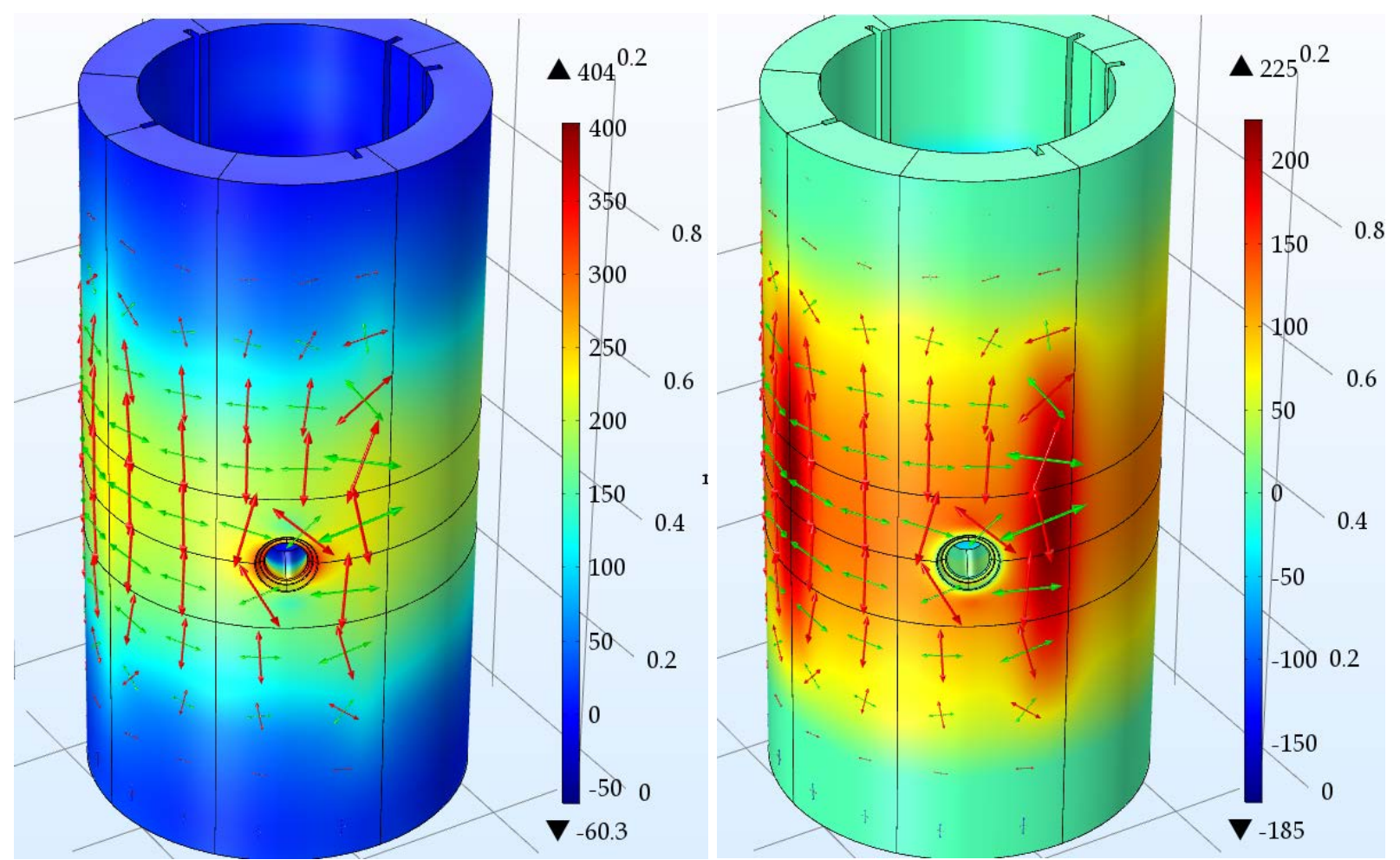

Figure 4.18. $1^{\text {st }}$ (left) and $2^{\text {nd }}$ (right) principal stress distribution with principal directions in beryllium reflector with beamport hole after 8 years of operation with HEU core 


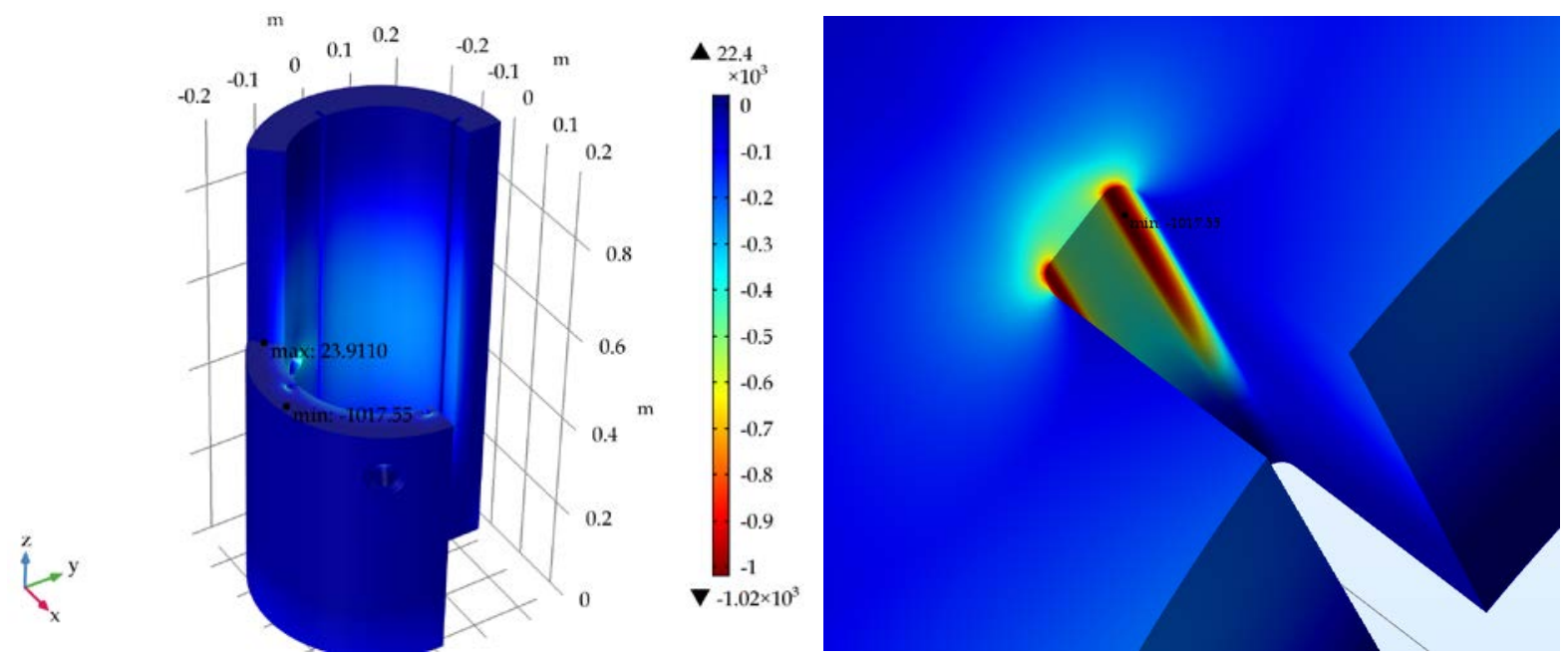

Figure 4.19. $3^{\text {rd }}$ principal stress distribution in beryllium reflector with beamport hole after 8 years of operation with HEU core
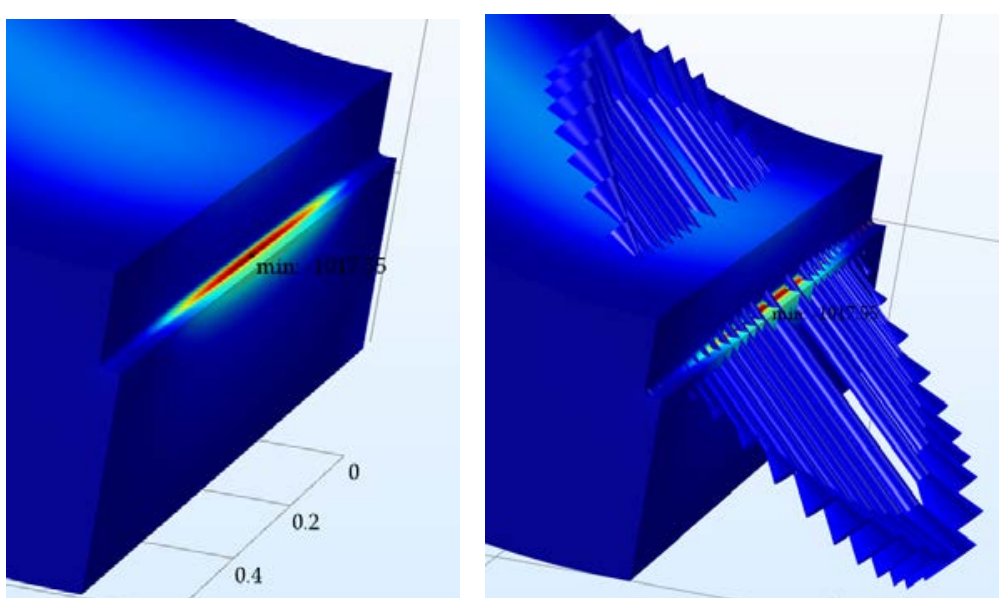

Figure 4.20. $3^{\text {rd }}$ principal stress distribution and principal direction in beryllium reflector with beamport hole after 8 years of operation with HEU core

\subsubsection{Stresses in Beryllium (S-200-FH) Reflectors with HEU Core and No Beamport Holes}

Figure 4.21 presents the distribution of temperature in the beryllium reflector without the beamport hole in the reactor operating with the HEU core for 8 years. This distribution is very similar to the one shown in Figure 4.14 for the reflector with the beamport hole (temperature variation is only present in the vicinity of the beamport holes). The peak temperature is predicted to be almost $368 \mathrm{~K}$. The right-hand panel of Figure 4.21 shows the distribution of temperature through the thickness of the reflector in the hottest location. Note that the maximum temperature refers to a point within the reflector body, not the surface exposed in the figure. The peak temperature is shifted closer to the inner surface of the reflector because of the shape of the heating function, which has a peak on the inner face of the reflector. 

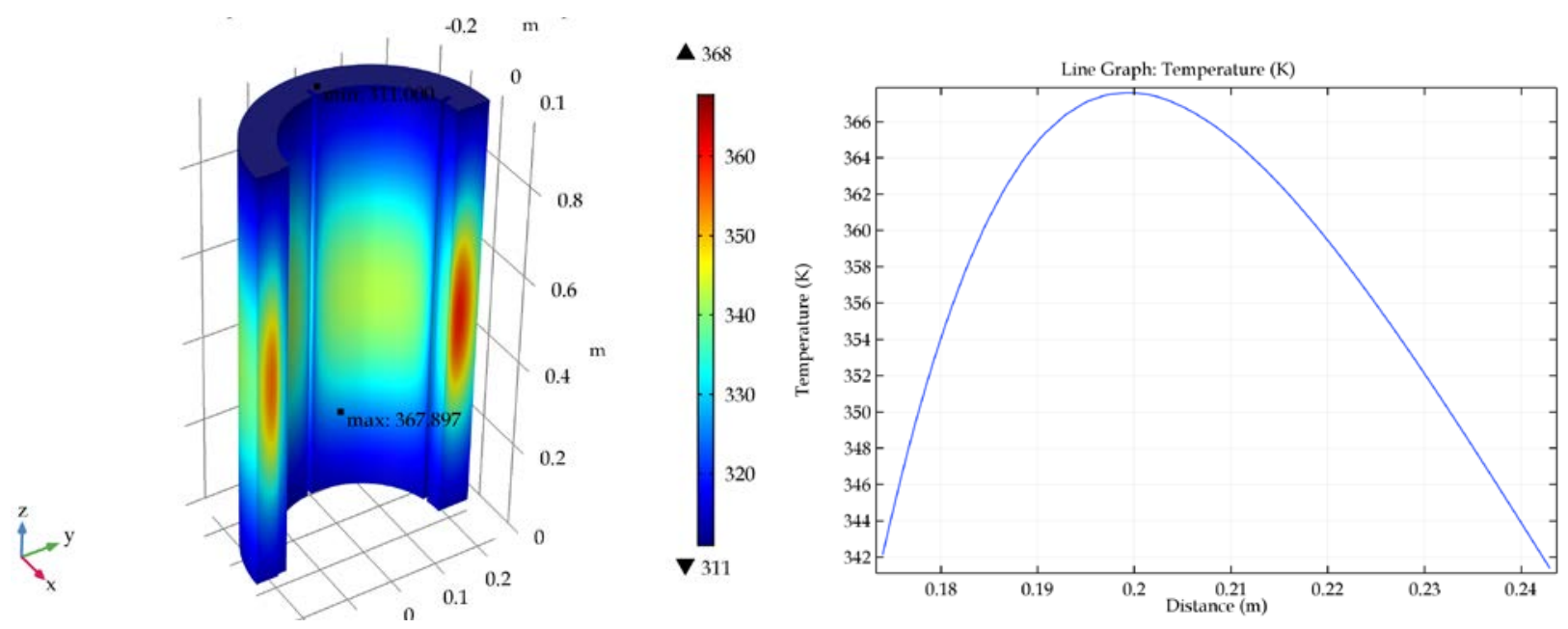

Figure 4.21. Temperature distribution in the FE model (left) and the distribution of temperature through the thickness of the reflector in the hottest location along the $y$-axis (right) in beryllium reflector without beamport hole after 8 years of operation with HEU core

Figure 4.22 shows the distribution of $1^{\text {st }}$ principal stress in the reflector without the beamport hole after 8 years of reactor operation with HEU fuel. In the right-hand panel, the ratio of these stresses to the local (degraded) UTS is displayed. The maximum $1^{\text {st }}$ principal stress at this point of operation is about $64 \%$ of the local, degraded UTS on the outer surface of the reflector.
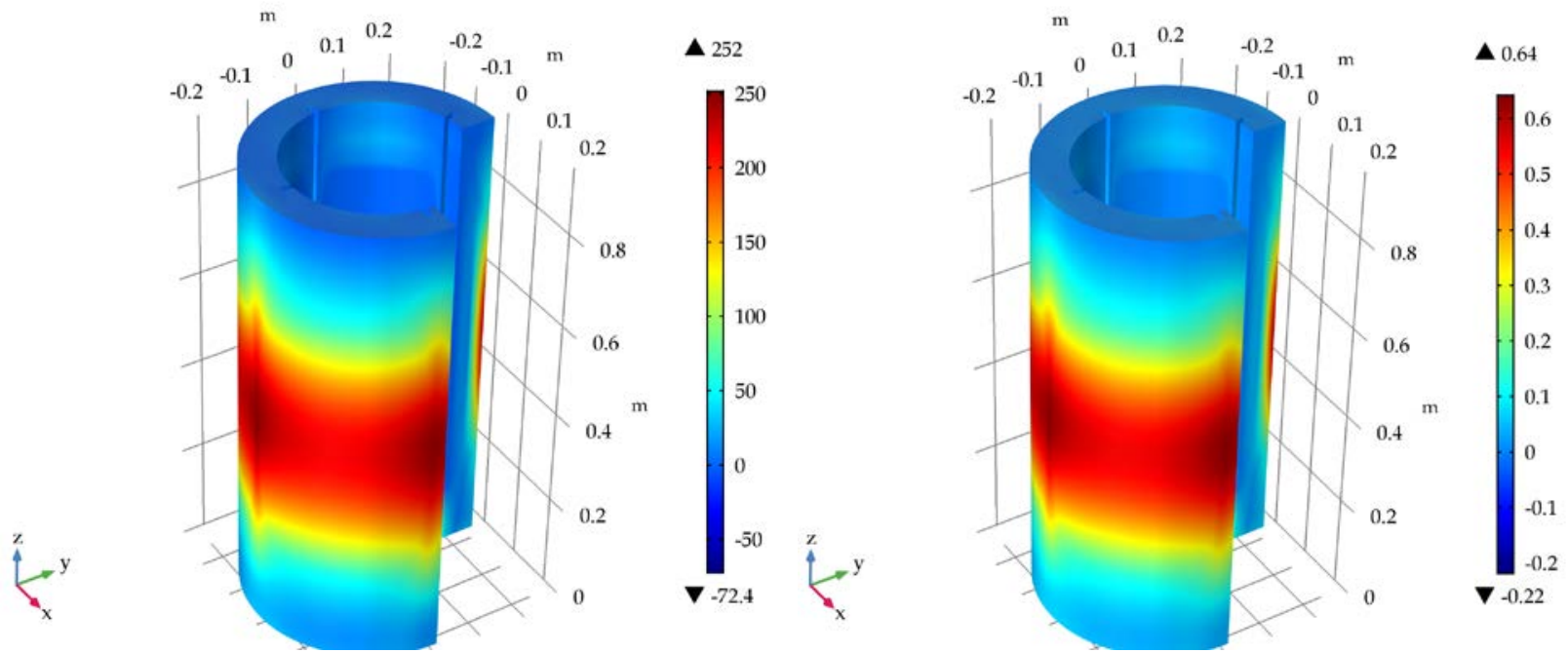

Figure 4.22. $1^{\text {st }}$ principal stress, MPa (left) and ratio of $1^{\text {st }}$ principal stress to local UTS (right) in beryllium reflector without beamport hole after 8 years of operation with HEU core

Figure 4.23 shows the distributions of the $2^{\text {nd }}$ and $3^{\text {rd }}$ principal stresses in the model of the reflector without the beamport hole operating for 8 years with an HEU core. The maximum $2^{\text {nd }}$ principal stress is tensile and is close in magnitude to the $1^{\text {st }}$ principal stress. The maxima of these two principal stresses are located at the same place, on the outer surface of the reflector behind the grooves and at the mid-height. The principal directions of stresses on the outer surface of the reflector are shown in Figure 4.24. The principal directions on the bottom of the grooves are shown in Figure 4.25. To assess the state of stress and margin to failure, the full state of stress needs to be considered and simple 
comparisons of the components to the UTS are not sufficient. Section 5 will attempt to interpret this state of stress in comparison to the two other analyzed models.

The $3^{\text {rd }}$ principal stress is near zero on the outer surface of the reflector and is the highest on the inner face, with a localized maximum on the bottom of the groove. Its distribution and magnitude are very similar to the case with beamport holes, as the same heating and swelling loadings are used in the analysis.
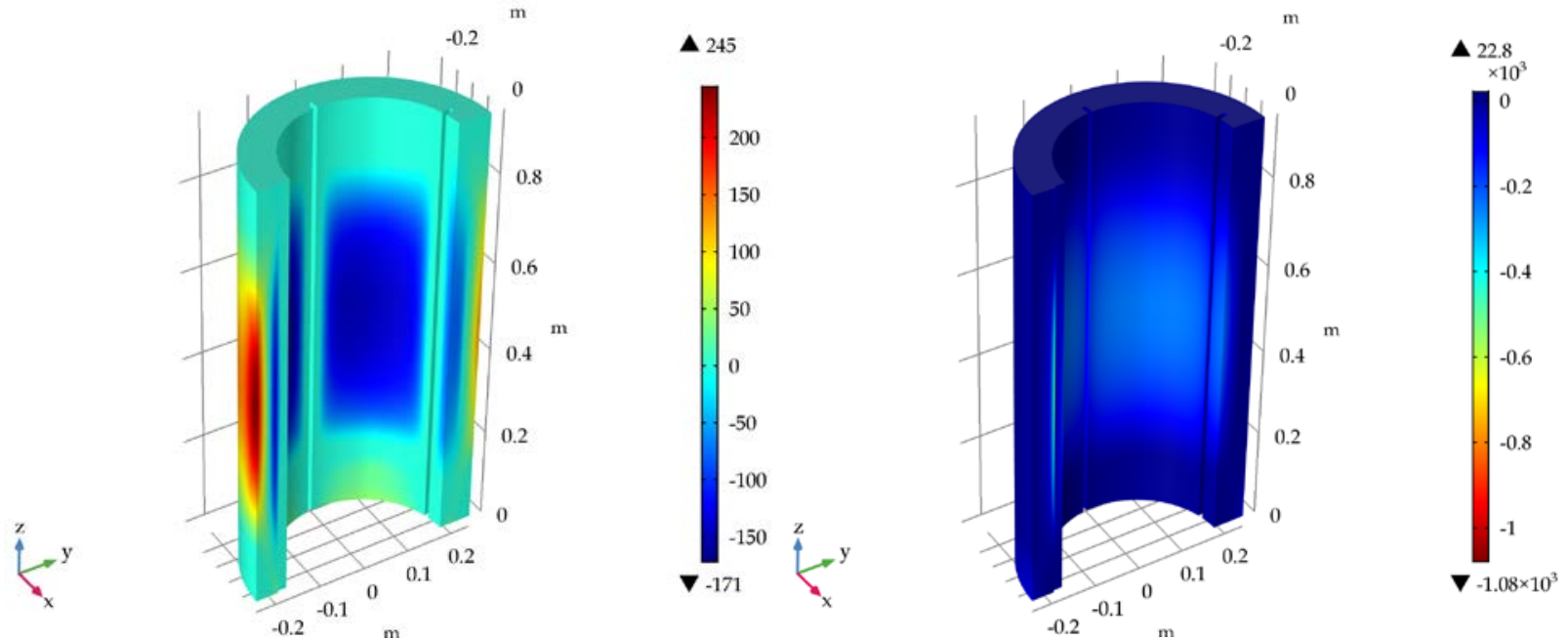

Figure 4.23. $2^{\text {nd }}$ (left) and $3^{\text {rd }}$ (right) principal stress, MPa, in beryllium reflector without beamport hole after 8 years of operation with HEU core

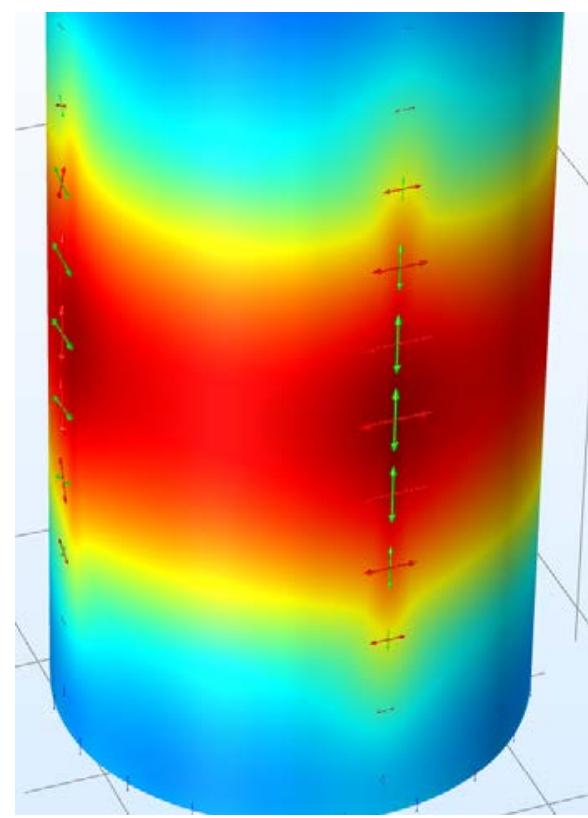

Figure 4.24. Principal directions of stresses on the outer surface of the beryllium reflector without beamport hole after 8 years of operation with HEU core 

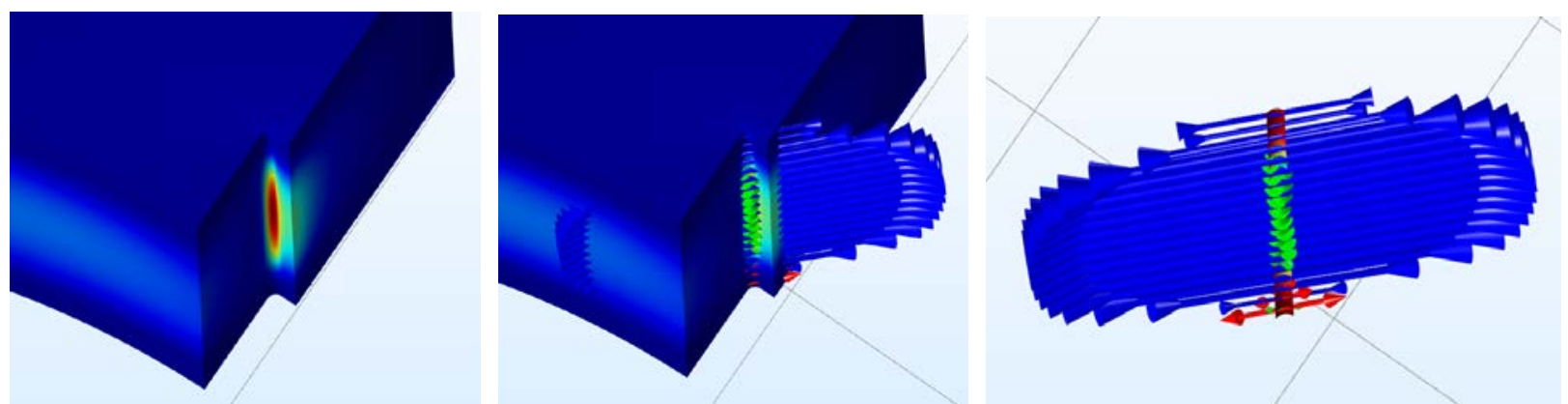

Figure 4.25. $3^{\text {rd }}$ principal stresses on the bottom of the groove, with their principal directions, in beryllium reflector without beamport hole after 8 years of operation with HEU core

\subsubsection{Stresses in Future Beryllium (S-200-FH) Reflectors with LEU Core and No Beamport Holes}

Figure 4.26 shows the distribution of the temperature in a beryllium reflector without a beamport hole at 8 years of operation with the LEU core. On the right, the temperature distribution through the thickness at the hottest location is plotted. The maximum temperature reaches about $362 \mathrm{~K}$, which is about $6 \mathrm{~K}$ lower than the temperature predicted for a reflector operating with the HEU core because of differences in the photon heating (refer to Section 2.4 for details).
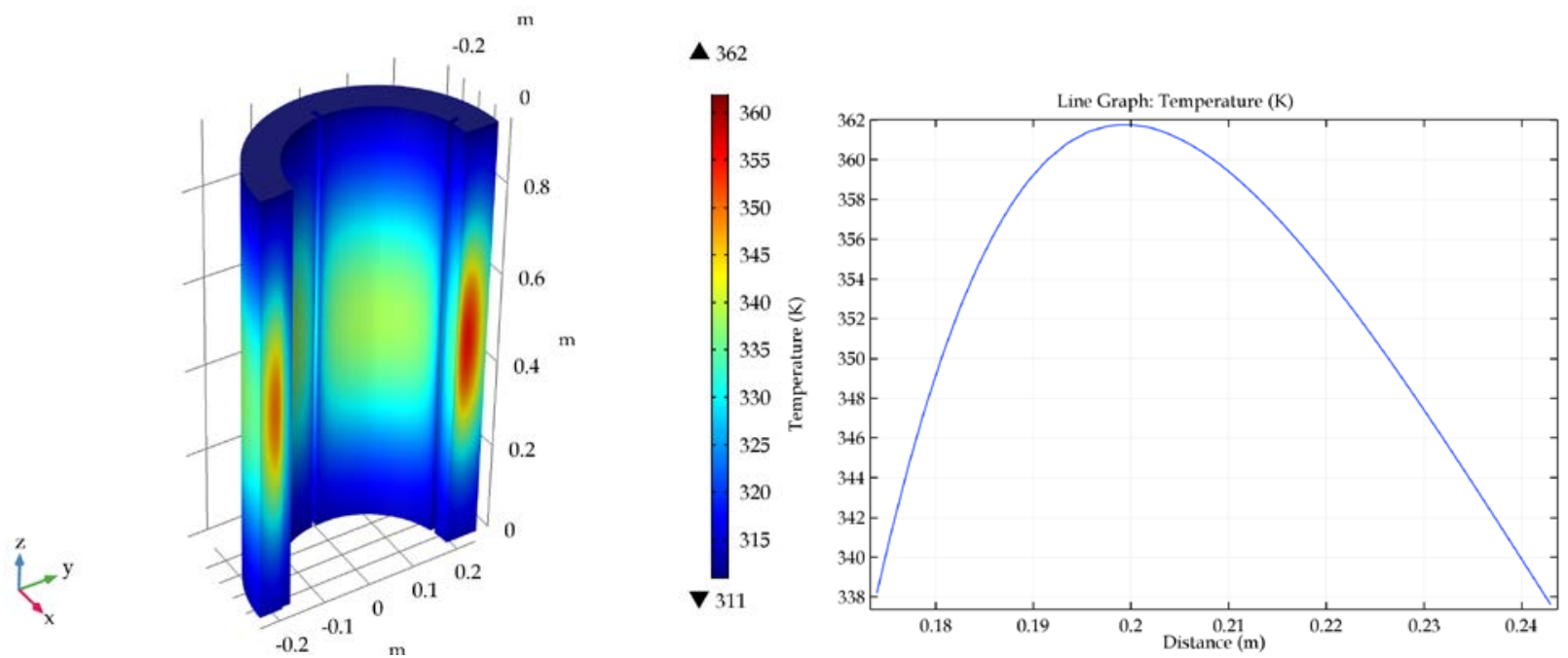

Figure 4.26. Temperature distribution in the FE model (left) and the distribution of temperature through the thickness of the reflector in the hottest location along $y$-axis (right) in beryllium reflector without beamport hole after 8 years of operation with LEU core

Figure 4.27 presents the distribution of the largest tensile principal stresses ( $1^{\text {st }}$ principal stress) in the beryllium reflector after 8 years of operation with the LEU core. The peak value reaches about $266 \mathrm{MPa}$. The areas with highest tensile stresses are located on the outer surface of the reflector, near the mid-height, behind the spacer grooves. These are the most critical locations for the current reflectors without beamport holes.

Figure 4.27 also presents the ratio of the $1^{\text {st }}$ principal stress to the local, degraded UTS. The maximum ratio is $68 \%$, as compared to $64 \%$ for the HEU-core case. The increase in this ratio is caused by a 
higher maximum stress in the LEU case (266 MPa vs $252 \mathrm{MPa}$ ) as well as a greater reduction of the local UTS due to slightly higher irradiation damage. This finding suggests that the beryllium reflector with the LEU core will have a shorter life than the reflector operating under the current conditions with the HEU core. Nonetheless, the maximum tensile stresses for the cases with LEU and HEU without a beamport hole are significantly lower than the stresses in the reflector with a beamport hole. Also, the strength of the S-200-FH beryllium grade that is used in current reflectors is substantially higher than the strength of the N-200-A grade used in the first reflector, which failed. Thus, the life of the reflectors without a beamport hole operating today with HEU cores at $10 \mathrm{MW}$ and in the future with LEU cores at $12 \mathrm{MW}$ is longer than the life of the reflector with beamport holes. The exact determination of how much longer the reflector can survive in the reactor without a failure is hard to predict. For that purpose, we look into the 3D state of stress, discuss the critical crack length for each of the analyzed cases, and examine potential crack propagation failure in the following section.
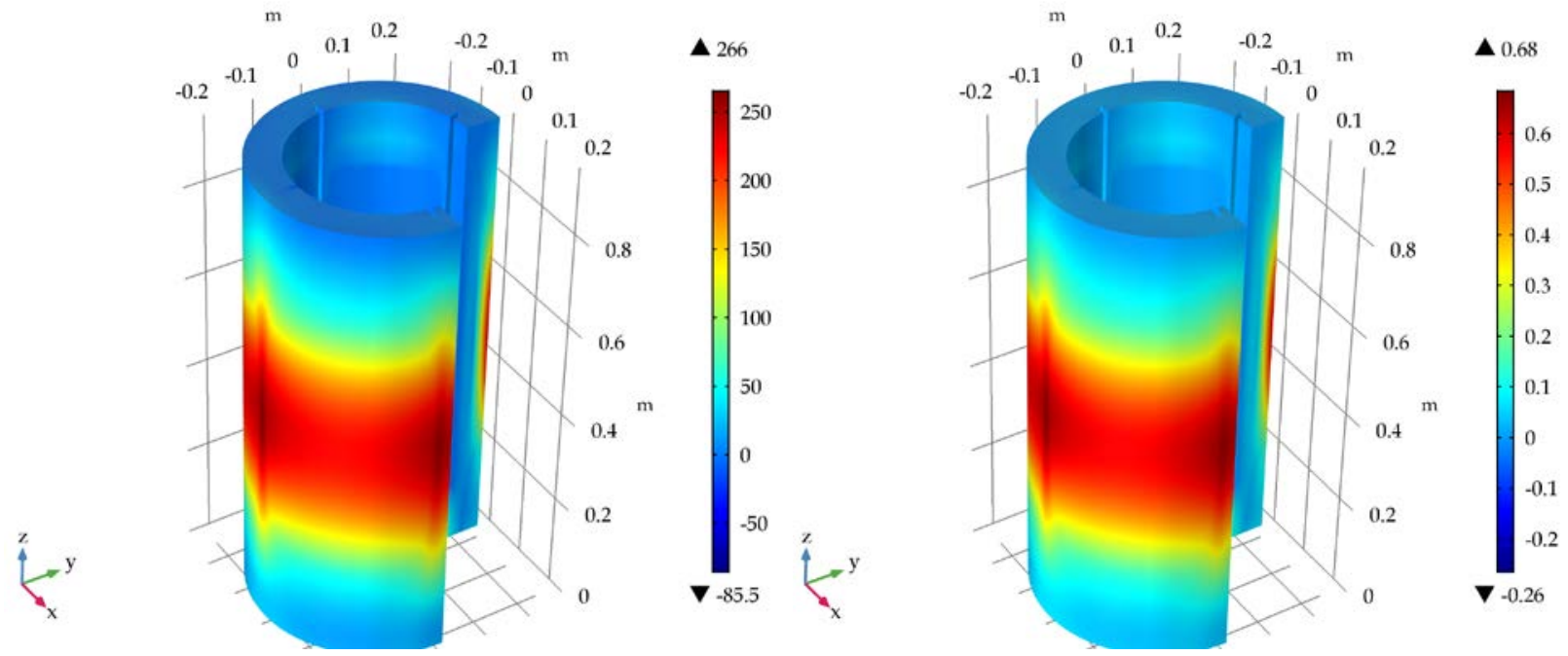

Figure 4.27. $1^{\text {st }}$ principal stress (left) and ratio of $1^{\text {st }}$ principal stress to local UTS (right) in beryllium reflector without beamport hole after 8 years of operation with LEU core

Figure 4.28 shows the distribution of $2^{\text {nd }}$ and $3^{\text {rd }}$ principal stresses in the reflector with the LEU core at 8 years of operation. The distribution of the $2^{\text {nd }}$ principal stress follows that of the $1^{\text {st }}$ principal stress. It is also a tensile stress and its magnitude is only slightly lower (by about 6\%) than the magnitude of the $1^{\text {st }}$ principal stress. Thus, as stated before, the 3D state of stress, rather than a simple comparison of the maximum tensile stress to the UTS, needs to be considered as a predictor of reflector lifetime. 

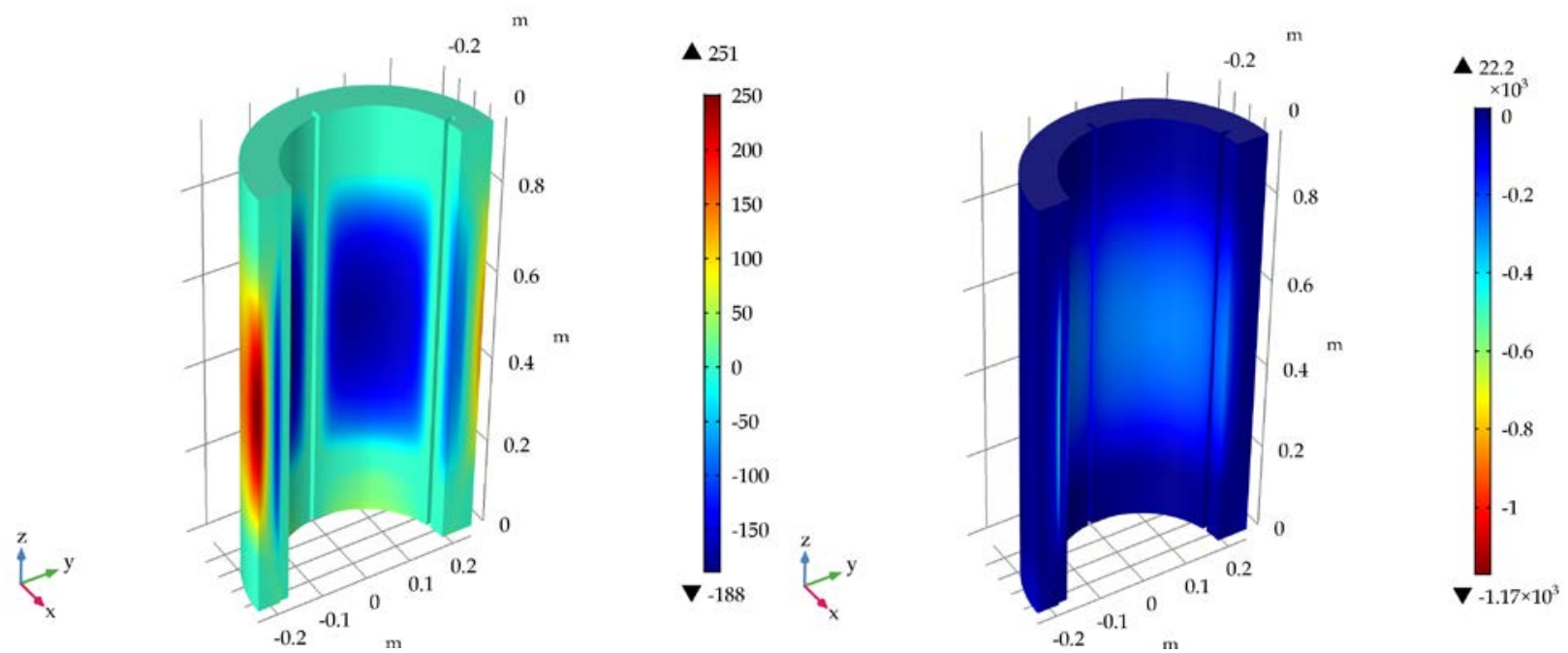

Figure 4.28. $2^{\text {nd }}$ (left) and $3^{\text {rd }}$ (right) principal stress in beryllium reflector without beamport hole after 8 years of operation with LEU core 


\section{Discussion}

\subsection{Tensile Stresses in Beryllium Reflectors}

Table 5.1 lists the maximum and minimum stresses from three combinations of stress contributors for the three models:

1. Beryllium reflector with 2 beamport holes operating with HEU core,

2. Beryllium reflector without beamport hole operating with HEU core, and

3. Beryllium reflector without beamport hole operating with LEU core.

The influence of heating alone at the beginning of life, swelling alone after 8 years of operation, and the combined influence of heating and swelling after 8 years of operation are presented for each of these models. The table only lists one component of the three principal stresses (i.e., the one with the largest magnitude) in a location of interest. From the comparison of maximum stresses (1 1 st principal tensile stress), it can be noted that the largest stresses are present in the model with the beamport hole. The first MURR reflector was identical to the second one that did not fail. It is possible that the model used in this study is overly conservative. It is also quite likely that there were fewer flaws in the highly stressed areas of the second reflector and that's why the failure of the second reflector did not occur. The third reflector had only one beamport hole. However, it was made out of a significantly stronger beryllium grade (S-200-FH). By removing the holes from subsequent reflectors, the stress concentration feature around the edge of the beamport hole was eliminated, substantially decreasing the maximum tensile stress. The maximum tensile stress in the model without a beamport hole operating with the HEU core is $252 \mathrm{MPa}$, which is a 38\% reduction of maximum stresses compared to the reflector with the beamport holes. As pointed out in Section 4.6, the location of the maximum tensile stress, when there is no beamport hole in the model, was near the mid-height behind the grooves on the outer surface of the reflector. For the reflector with beamport holes, the stresses at these locations were the same. Thus, the initiation of the failure that occurred in the first reflector is attributed to the stress concentrations near the beamport holes. The substantially lower strength of the older N-200-A grade of beryllium, as compared to the strength of the current S-200-FH grade, is another factor contributing to that failure. Ductility and thermal conductivity are also higher for the S-200-FH grade, contributing to its longer life as compared to the N-200-A grade.

In the model operating with the LEU core, the maximum tensile stresses have increased to $266 \mathrm{MPa}$ (5.5\% greater than the HEU core case) because of a larger concentration of He-4 following conversion and a power uprate from $10 \mathrm{MW}$ with HEU to $12 \mathrm{MW}$ with LEU. However, these stresses with the LEU core are 34\% lower than the $404 \mathrm{MPa}$ predicted near the beamport holes for the first reflector with an HEU core.

Table 5.1. Maximum stress values in reflectors (MPa) with base properties

\begin{tabular}{|c|c|c|c|c|c|c|}
\hline & \multicolumn{2}{|c|}{ Heating only at 0 years } & \multicolumn{2}{c|}{ Swelling only at 8 years } & \multicolumn{2}{c|}{$\begin{array}{c}\text { Heating and swelling at 8 } \\
\text { years }\end{array}$} \\
\cline { 2 - 7 } & $\begin{array}{c}\text { Max stress } \\
\text { (tension) }\end{array}$ & $\begin{array}{c}\text { Min stress } \\
\text { (compression) }\end{array}$ & $\begin{array}{c}\text { Max stress } \\
\text { (tension) }\end{array}$ & $\begin{array}{c}\text { Min stress } \\
\text { (compression) }\end{array}$ & $\begin{array}{c}\text { Max stress } \\
\text { (tension) }\end{array}$ & $\begin{array}{c}\text { Min stress } \\
\text { (compression) }\end{array}$ \\
\hline HEU with BP & 88.5 & -32.3 & 270.0 & $-1,013$ & 403.9 & $-1,018$ \\
\hline HEU, no BP & 60.2 & -29.5 & 199.7 & $-1,066$ & 251.9 & $-1,079$ \\
\hline LEU, no BP & 44.9 & -25.7 & 218.7 & $-1,166$ & 265.8 & $-1,171$ \\
\hline
\end{tabular}

Table 5.2 lists the ratios of maximum tensile stress (single component only) to the local, degraded UTS for the three analyzed cases. For the case of the reflector with the beamport holes, the maximum ratios at the edge of the beamport entrance and on the outer surface of the reflector behind the spacer 
grooves are listed. A ratio greater than 1.0 means that the degraded UTS is exceeded and failure may occur. For the reflector with the beamport holes, there were nine locations (five grooves, two beamport holes with two sides) where the UTS was exceeded or the stresses were very close to it (see Figure 5.1 for the location of the highest stresses in the reflector). This situation could easily lead to a propagation of cracks on the entire annulus and produce a failure like that observed in 1981.

The value of the stress-to-UTS ratio, 1.61, may indicate that the model used in this analysis is overly conservative. However, it must be noted that this model assumes elastic properties of the beryllium only while the transition from ductile to brittle occurs slowly in time with increasing fluence. The UTS of beryllium could have been reached near the beamport holes sooner (after less than 8 years of equivalent operation at $10 \mathrm{MW}$ ). Local yielding could have occurred, relieving the stresses. The models presented here are mostly intended for estimation of a relative life of the beryllium reflector operating in the future with an LEU core as compared to the historically and currently operating reflectors with HEU cores.

Table 5.2. Ratio between the maximum stresses and local UTS of beryllium material in various models

\begin{tabular}{|c|c|c|c|c|c|}
\hline Fuel & $\begin{array}{c}\text { Core Power } \\
\text { (MW) }\end{array}$ & Beamport & $\begin{array}{c}\text { Beryllium } \\
\text { grade }\end{array}$ & $\begin{array}{c}\text { Location of } \\
\text { stresses }\end{array}$ & $\begin{array}{c}\text { Max } \\
\text { ratio }\end{array}$ \\
\hline HEU & 10 & Yes (two) & N-200-A & Beamport hole edge & 1.61 \\
\hline HEU & 10 & Yes (two) & N-200-A & Behind grooves & 0.93 \\
\hline HEU & 10 & No & S-200-FH & Behind grooves & 0.64 \\
\hline LEU & 12 & No & S-200-FH & Behind grooves & 0.68 \\
\hline
\end{tabular}
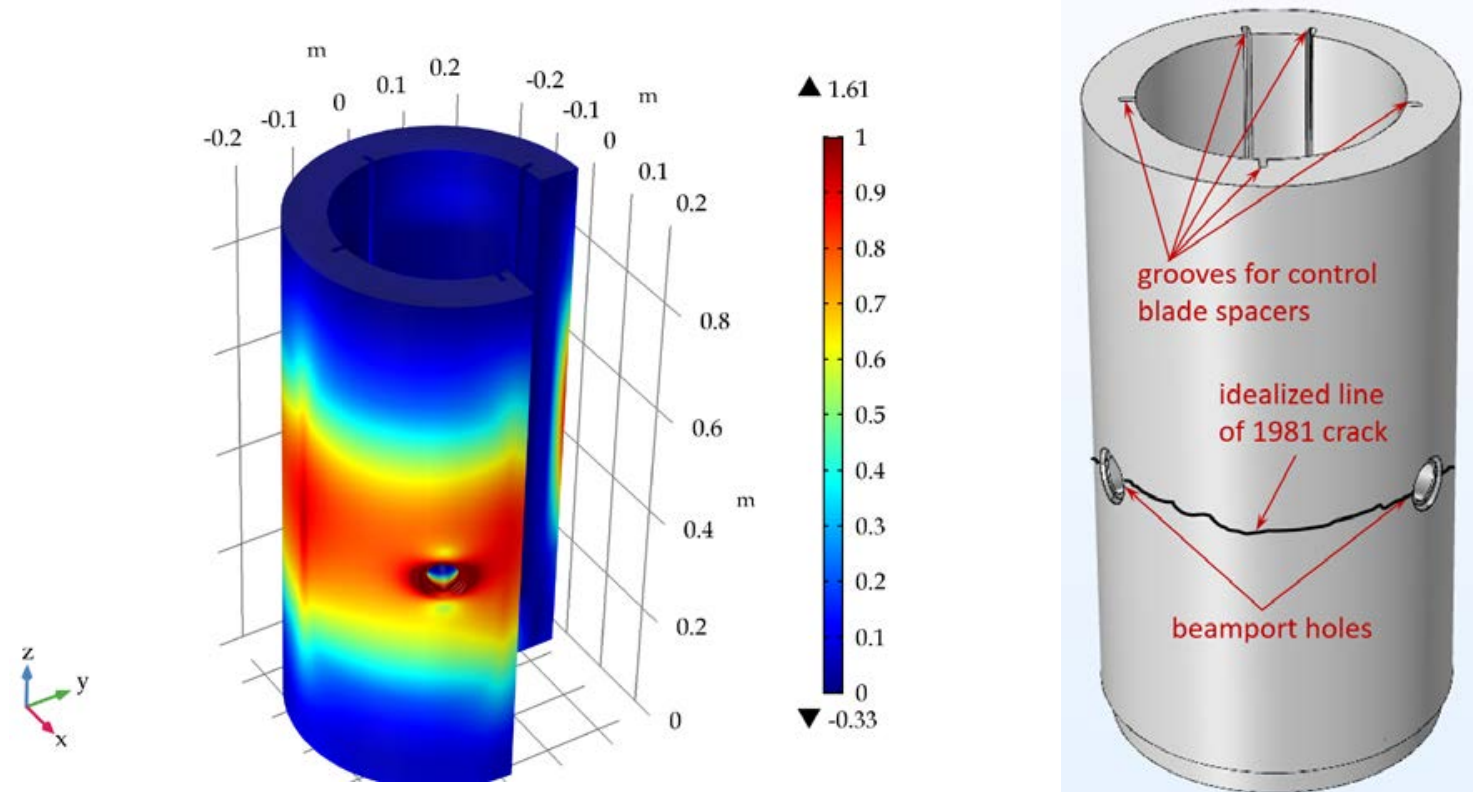

Figure 5.1. Locations of highest stresses in the reflector with beamport holes (left) and schematic of crack location in reflector from 1981 (right)

The beryllium reflector without the beamport holes operating with the LEU core is predicted to experience larger stresses than the same reflector operating with the HEU core. Thus, its life is expected to be shorter than that of the current reflector with the HEU core. The analysis required multiple assumptions regarding the boundary conditions, loadings, and material properties. The authors recognize that with so many unknowns, it is valuable to understand whether there is a set of 
unfavorable conditions that could lead to a failure of the reflector with the LEU core sooner than the current operation time of 8 years. Figure 5.2 provides a schematic of the progression of stress growth and degradation of the UTS with time for the three analyzed cases. The orange, green, and blue solid lines represent the increase in stress for the beryllium reflectors/cores that have been evaluated. The stress for the reflector with the beamport hole (orange line) starts from a higher level than those for the reflectors without beamport holes (green line for HEU and blue line for LEU). These initial values correspond to the thermally induced stresses for each case, which are summarized in Table 5.1. The blue curve corresponding to the LEU core starts from the lowest value because the reflector heating is lower with the LEU core. Subsequent growth in the stress for this reflector case is mostly due to the increased swelling with irradiation. Additional thermal stresses are produced because of the degrading thermal conductivity. At some point in time, the stress in the reflector with the LEU core exceeds that in the corresponding reflector with the HEU core. However, the difference between these two cases remains very small. After 8 years of operation, the maximum tensile stresses for the reflector with the HEU and LEU cores are predicted to be $252 \mathrm{MPa}$ and $266 \mathrm{MPa}$, respectively.

If any of the analysis assumptions regarding the thermal- or swelling-induced stresses lead to underpredicting of the total stress, these assumptions will affect all the cases in a similar manner. Thus, the starting point of the stress lines and/or the slope of the lines in Figure 5.2 would change for different assumptions, but the change would be similar for all three cases. That said, there is high confidence that for the MURR reflector, within a reasonable operation time (around 8 years), which was selected as a safe operation time for the reflector with beamport holes, the stresses in reflectors without beamport holes will not be higher than the stresses predicted for the reflector with beamport holes.

The dashed lines in Figure 5.2 represent the change in the UTS of the beryllium materials with time. It is unknown how much error is associated with the assumed degradation profiles. It is known, however, that the failed reflector was built from a weaker grade of beryllium than the current reflector. Similarly to the above discussion on the predicted stress, within a reasonable operation time (around 8 years), the UTS of N-200-A is expected to remain lower than the UTS of S-200-FH irradiated with the LEU core. However, since the beryllium material damage rate with the LEU core will be slightly higher than for the reflector operating with the HEU core, it is reasonable to maintain the assumption that the UTS of the reflector with the LEU core will always be lower than that for the reflector with the HEU core. Thus, the replacement schedule for the reflector with the LEU core should not be longer than that of the reflector with the HEU core. 


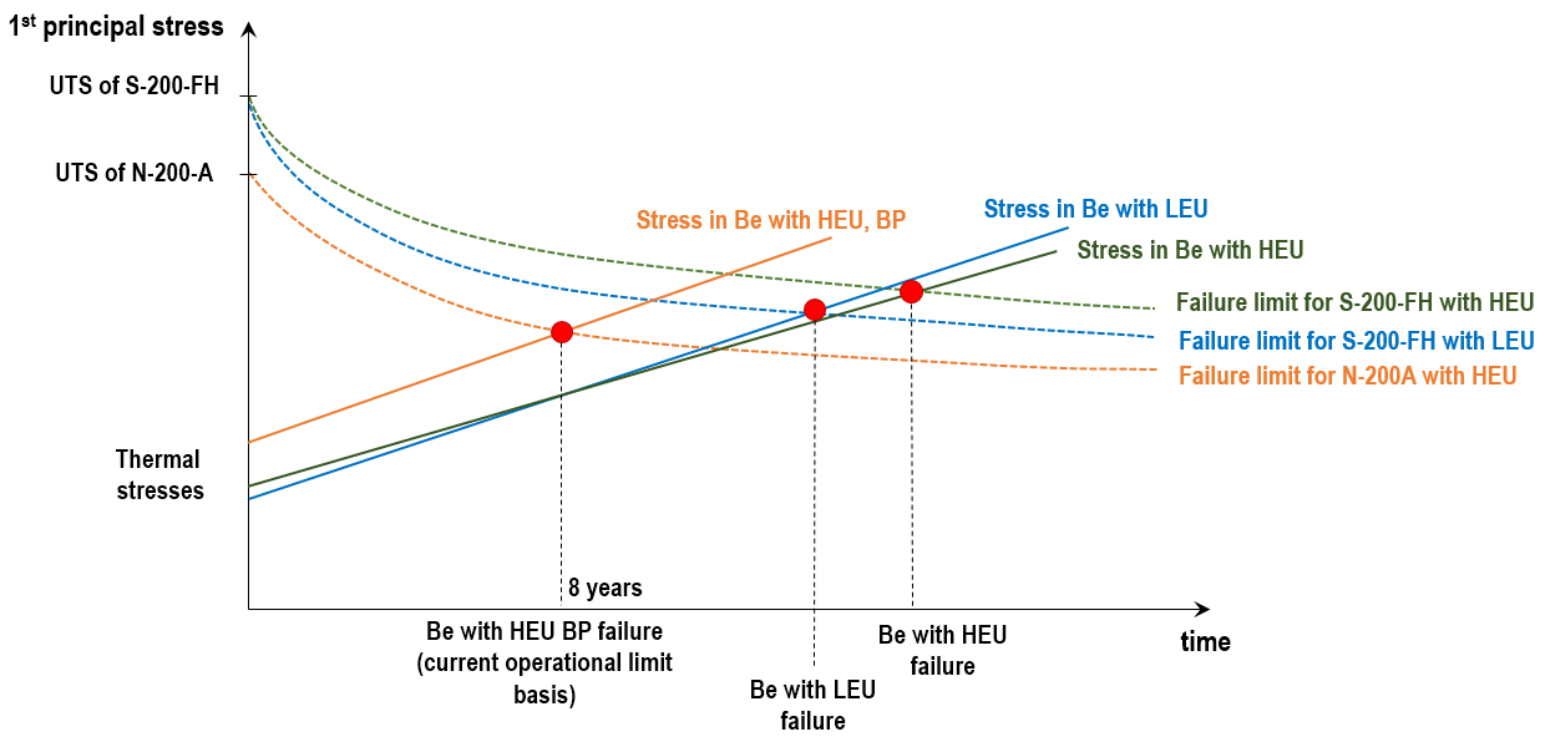

Figure 5.2. Relative life prediction for beryllium reflectors with HEU and LEU cores. Note that the plot is not drawn to scale and has only a qualitative character.

The intersection of each solid line with the corresponding dashed line of the same color in Figure 5.2 indicates a point when the UTS is exceeded by the stress in the reflector, meaning a failure of the reflector could occur at any time after that. It is concluded that for the reasons given above, the stressinduced failure of the reflector operating with the LEU core will occur sooner than for the same reflector (design, material) operating with the HEU core. However, it is highly unlikely that this failure would occur before 8 years of operation or at a time before the failure of the reflector with the beamport hole.

As mentioned earlier, to fully understand the relative life of the reflectors for the three cases that were analyzed, the overall state of stress needs to be considered instead of just one component of it. The points on the outer surface of the reflector, including the ones on the edge of the beamport hole, have two significant principal-stress components that are in tension, while the third principal stress remains relatively small and close to zero. Thus, further analysis of a $2 \mathrm{D}$ principal stress space is justified.

Figure 5.3 shows failure loci according to the Maximum Normal Shear stress, Coulomb-Mohr, and Christensen criteria together with the stresses in the most stressed point in the beryllium reflector with the beamport hole at 8 years of operation. Two points, one on the edge of the beamport hole and another on the outer surface behind the groove at mid-height that is far from the beamport hole, are considered. The values of the $1^{\text {st }}$ and $2^{\text {nd }}$ principal stresses at these locations determine the positions of the points marked in the plot in Figure 5.3. The blue point, corresponding to the state of stress around the beamport hole, is outside the failure loci. The purple point, representing the state of stress behind the grooves near the mid-height, is very close to the locus of Maximum Normal Stress and well beyond the Christensen-criterion locus.

In general, it is possible that the individual stress components do not reach UTS, while in the 2D or 3D state of stress the failure state is reached. The Christensen failure criterion allows for such cases. However, it was shown in Section 3.5 to be too conservative in the first quadrant (where both principal stresses are in tension). Nevertheless, it is shown here as a reminder that the true failure locus may be located somewhere between the ones produced by the Christensen and the Maximum 
Normal Stress criteria. This determination can only be made upon performing biaxial loading tests for S-200-FH grade.
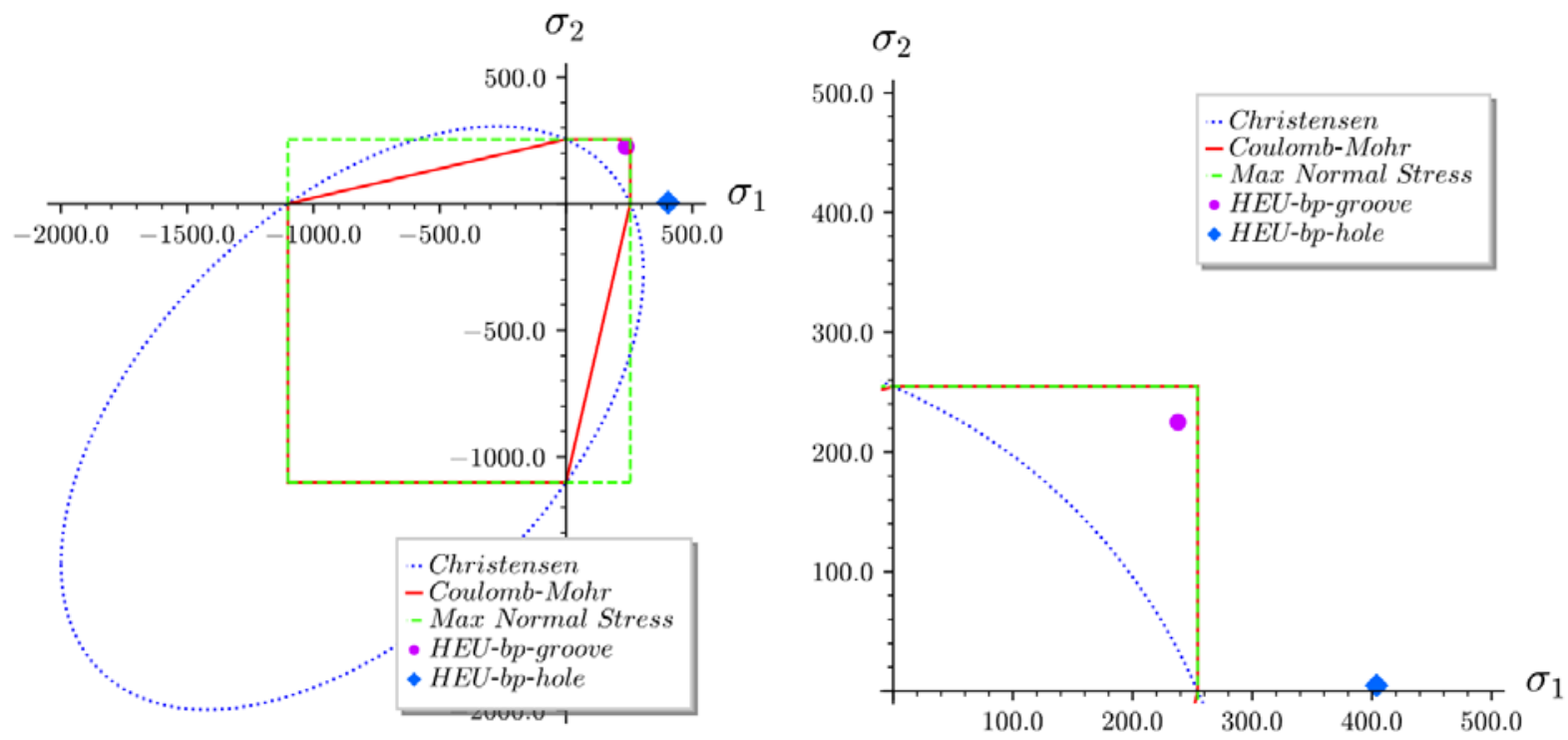

Figure 5.3. Location of points with highest tensile stresses in beryllium reflector with beamport hole in 2D principal stress after 8 years of operation with HEU core

Figure 5.4 and Figure 5.5 show failure loci for the beryllium reflector geometries without beamport holes, operating with HEU and LEU cores, respectively. The locus for the LEU case is slightly closer to the origin of the axes, as the strength degradation of beryllium is greater at equivalent operation times. The points representing the state of stress on the outer surface of the reflector behind the grooves are marked as blue circles. For both these cases, the points are well within the locus defined by the Maximum Normal Stress criterion. The point for the LEU case is slightly closer to the boundaries than the one for the HEU case. The Christensen's criterion locus is reached in both cases.
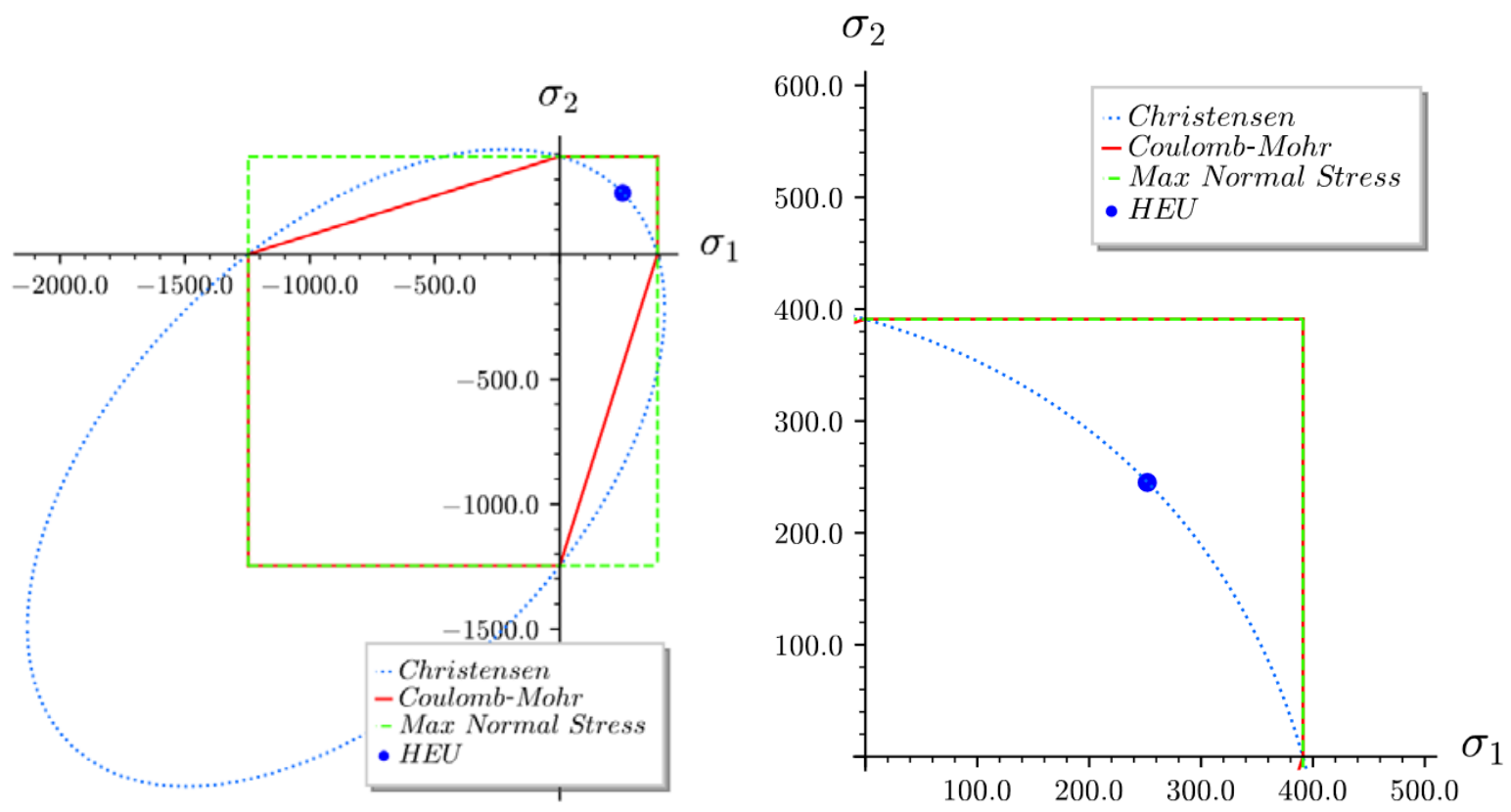

Figure 5.4. Location of points with highest tensile stresses in beryllium reflectors without beamport hole in 2D principal stress after 8 years of operation with HEU cores 


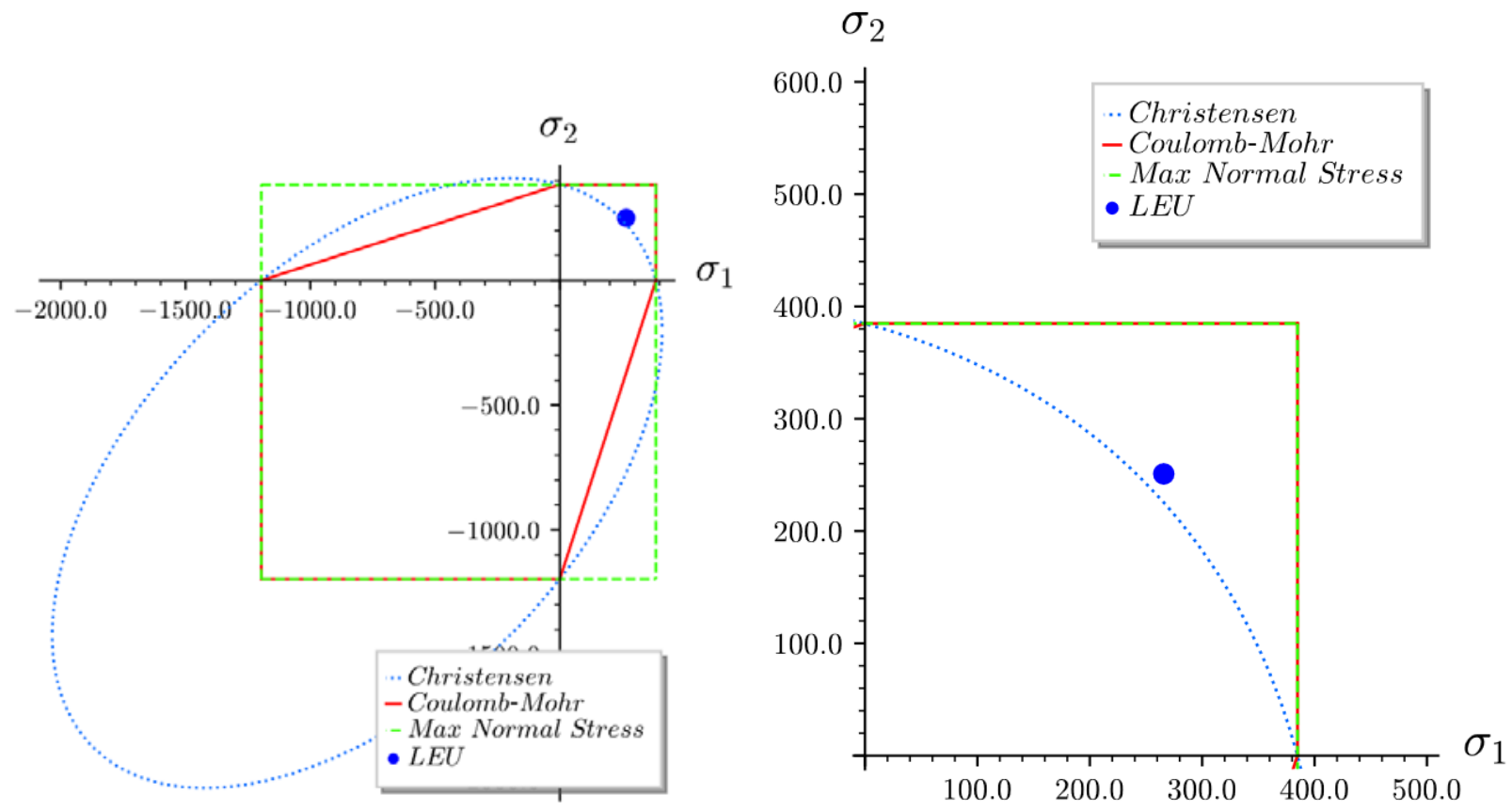

Figure 5.5. Location of points with highest tensile stresses in beryllium reflectors without beamport hole in 2D principal stress after 8 years of operation with LEU cores

As mentioned in Section 4.5, additional analysis has been performed with modeling uncertainties included. Table 4.7 lists the uncertainties that have been considered in the models. Figure 5.6, Figure 5.7, and Figure 5.8 show the progression of maximum stresses with time of operation in the three analyzed cases of reflectors. Figure 5.6 shows the progression of maximum stresses in time in the first two beryllium reflectors made out of N-200-A grade. Solid black and grey lines represent the increase of maximum stresses near the beamport holes and behind the grooves on the outer surface of the reflector, respectively. The dashed lines of the same colors represent the stresses predicted in the models with uncertainties included. The blue line represents the degrading UTS of N-200-A beryllium with time. Note that this model is only based on the minimum guaranteed UTS of N-200-A. The actual mean strength was higher than that, but there are no available data to estimate it. Thus, this fit is conservative. When one of the grey or black lines crosses the blue line, it means that the stresses at that location at that specific point in time have reached the UTS. As discussed earlier, the stresses near the beamport holes at 8 years have already been higher than the reduced UTS. According to the current model, the UTS was reached at about 4.5 years of operation near the beamport holes. Obviously, local yielding (which is not modeled here) could have occurred and could lead to redistribution of stresses and reduction of maximum stresses. Also, if the ductility of the material was entirely lost, cracks could have started to develop. According to the current model, the maximum tensile stresses behind the grooves could reached the UTS at about 8.5 years of operation. The uncertainties included in the model reduce these times to 3.7 and 7.4 years, respectively (cross section of dashed lines with the blue line). 


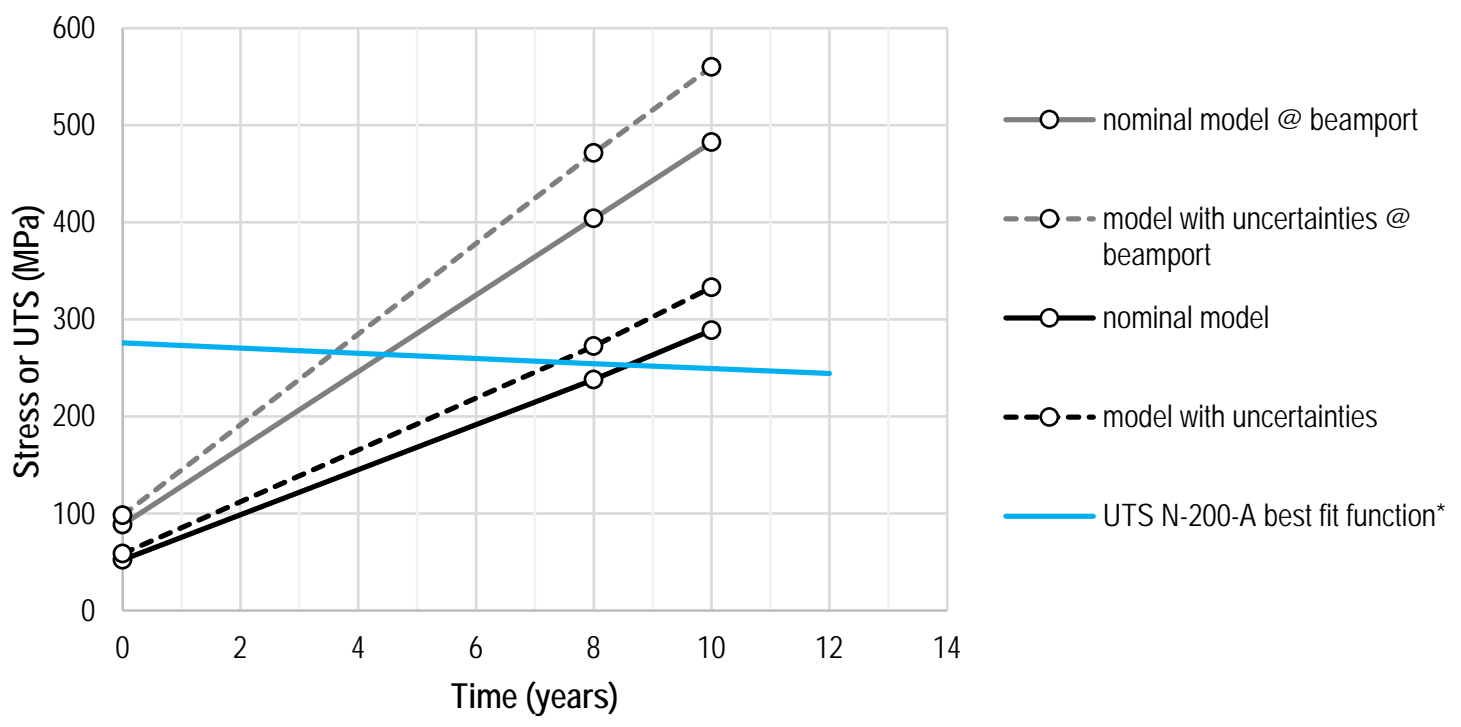

Figure 5.6. Progression of maximum stresses with assumed operation time of beryllium reflector (N-200-A) with beamport holes and HEU core

Figure 5.7 presents a similar analysis for the case of beryllium reflectors without beamport holes, made of S-200-FH grade and operating with the HEU core. Significantly more information about the strength of S-200-FH grade was found than for the N-200-A grade (refer to Section 3). That information allowed for building statistical models with the mean fit to UTS data (solid red line), the lower-bound 95\% confidence interval of the fit (dashed red line), and confidence intervals for individual samples (blue lines). The solid black line represents the maximum stresses behind the grooves at mid-height in the best-estimate model. The dashed black line represents the maximum stresses' progression in time for the model with uncertainties. The best-estimate model crosses the mean UTS line at about 12.2 years of operation. The maximum stresses in the model with uncertainties reach the 95\% lower confidence interval fit to UTS data (dashed black line crossing dashed red line) at slightly over 10 years. It is still debatable whether the confidence interval lines for UTS of individual samples (blue solid lines) should be used for life estimations or the mean fits. If the 95\% confidence interval for individual samples is used, the predicted stresses in the model with uncertainties will reach the UTS level at about 7.5 years of operation. Since all reflectors made of S200-FH beryllium survived 8 years of operation, this would most likely be an overly conservative estimation. 


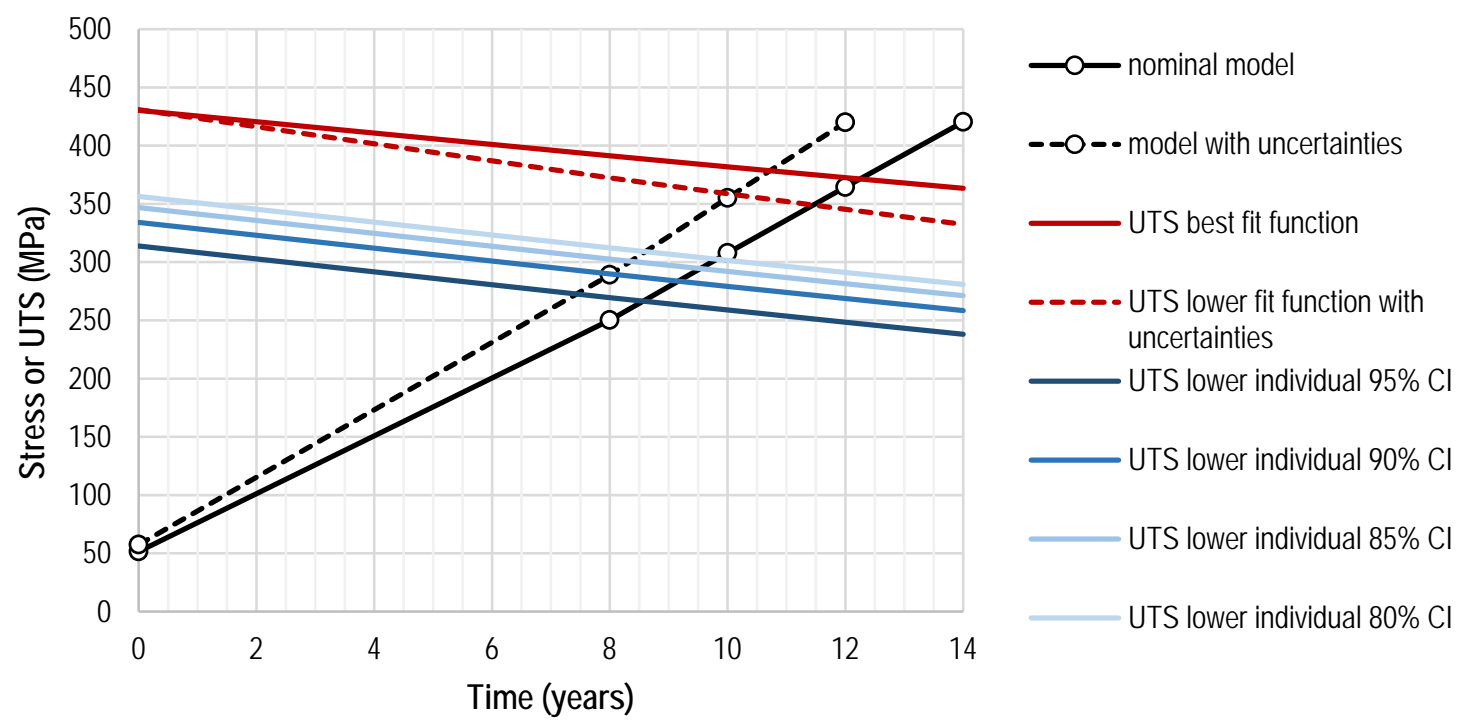

Figure 5.7. Progression of maximum stresses with assumed operation time of beryllium reflector (S-200-FH) without beamport holes and with HEU core

Figure 5.8 presents a similar analysis for the case of beryllium reflectors without beamport holes, made of S-200-FH grade and operating with an LEU core. The best-estimate model crosses the mean UTS line at about 11.3 years of operation. The maximum stresses in the model with uncertainties reach the 95\% lower confidence interval fit to UTS data (dashed black line crossing dashed red line) at around 9.3 years. If the $95 \%$ confidence interval on individual samples is used, the predicted stresses in the model with uncertainties will reach the UTS level at about 6.9 years of operation.

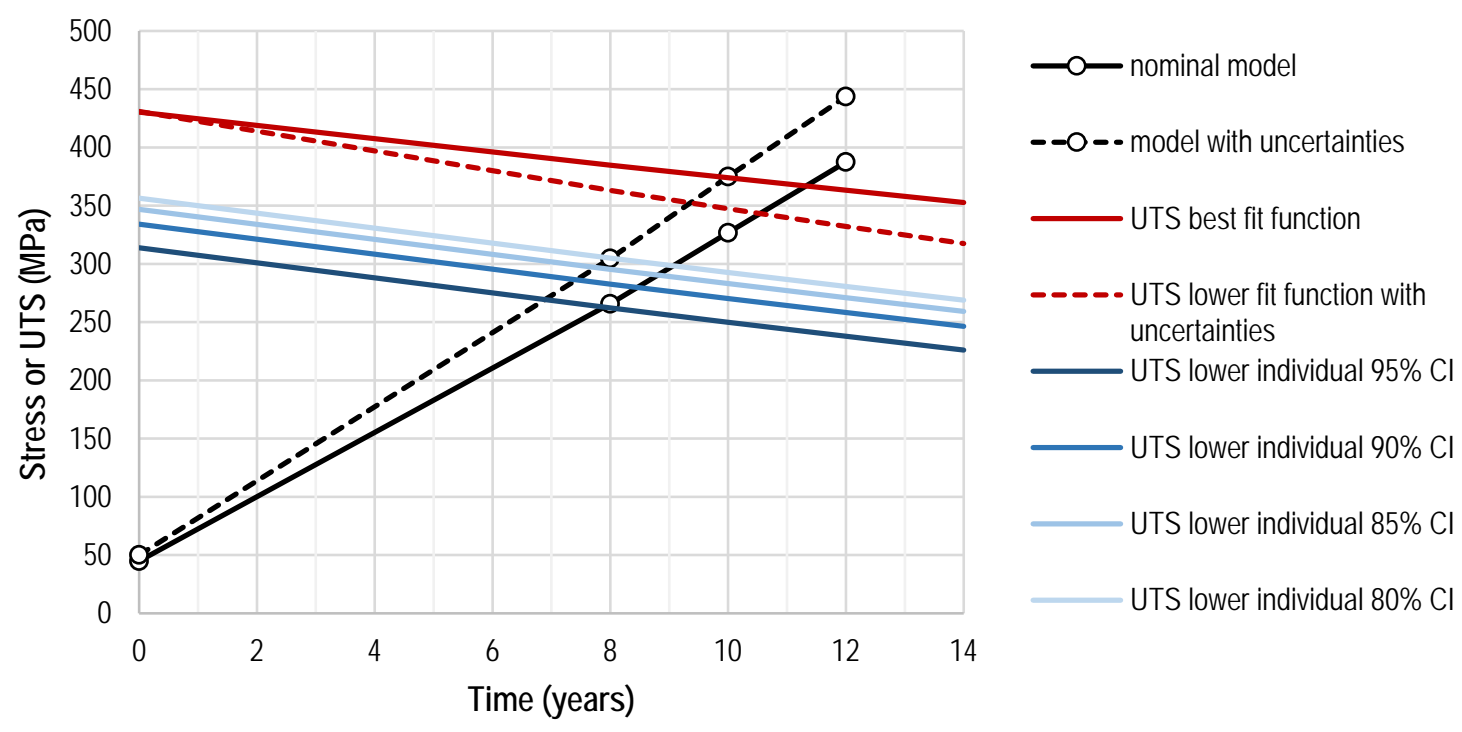

Figure 5.8. Progression of maximum stresses with assumed operation time of beryllium reflector (S-200-FH) without beamport holes and with LEU core

It must be reiterated that these plots pertain only to a single component of stress, i.e., the maximum tensile stresses on the outer surface of the reflector. The combination of other stresses as well as surface condition need to be considered simultaneously in a broader estimation of failure. 


\subsection{Compressive Stresses in Beryllium Reflectors}

While the tensile stresses are believed to be the cause of failure of the beryllium reflector and in general are more critical for brittle materials, the compressive stresses on the bottom of the grooves require careful examination and analysis as well. It was already mentioned in Section 4.6 that these stresses are very high, and the highest values were calculated for the LEU-core case. Figure 5.9 shows how the $3^{\text {rd }}$ principal stresses change through the thickness of the reflector in the vicinity of the spacer groove. These stresses are plotted along several lines, as marked on the left-hand portion of the figure. The colors of the curves in the plot correspond to the colors of the cross sections shown on the left. It can be noted that in the middle of the groove, the maximum compressive stress reaches almost $-600 \mathrm{MPa}$ (the cyan and blue solid curves). Near the fillet on the bottom of the groove, the stresses reach the level of $-1,100 \mathrm{MPa}$. The magenta cross-section line was made less than $1 \mathrm{~mm}$ beyond the groove's boundary. At this location, the maximum $1^{\text {st }}$ principal stresses decreased back to the level of $-600 \mathrm{MPa}$. Further away from the groove, the stresses decrease even more. In the cross sections away from the groove, such as the one marked with the dashed teal line $(\sim 40 \mathrm{~mm}$ from groove center), the stresses have a monotonic character with a maximum of about $-270 \mathrm{MPa}$ on the inner surface of the reflector and decrease to zero on the outer surface of the reflector. This behavior is common to all analyzed cases. Only the magnitude of the largest compressive stresses varies slightly among them.

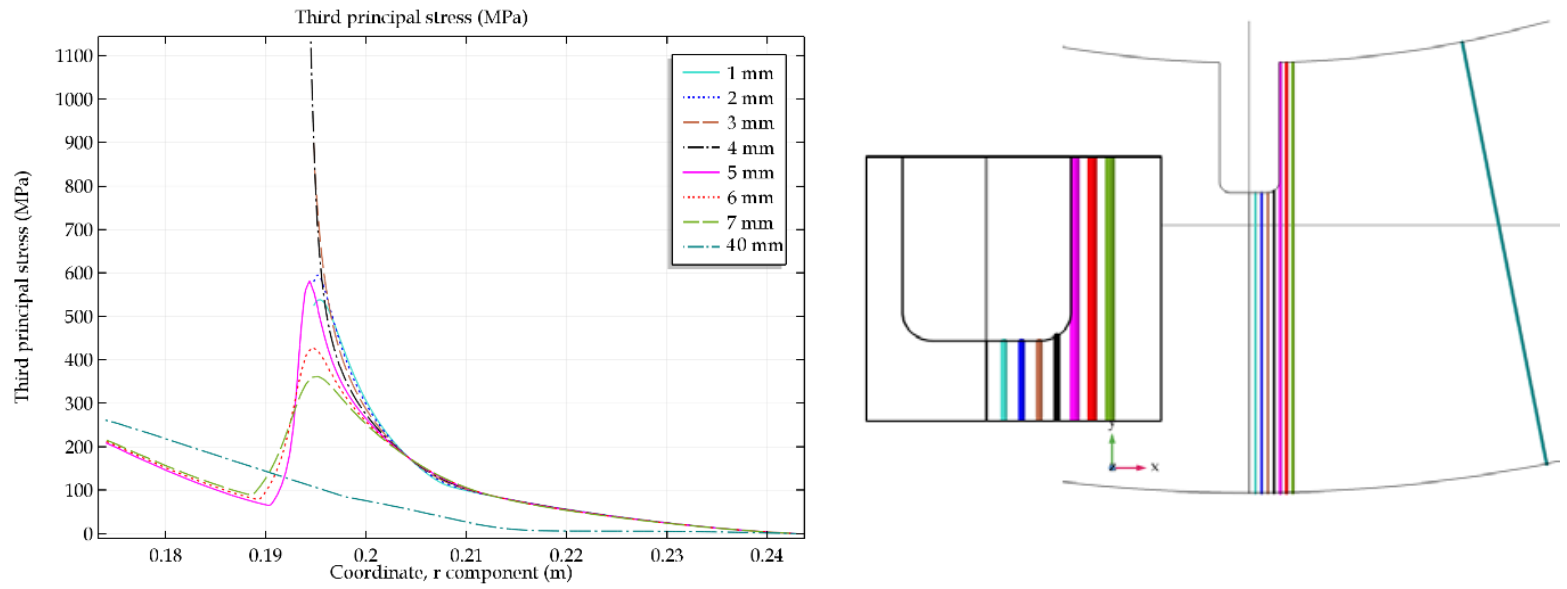

Figure 5.9. $3^{\text {rd }}$ principal stress distribution through the thickness of the beryllium reflector with the LEU core, in the vicinity of the spacer groove

Table 5.3 shows how the highest compressive stresses ( $3^{\text {rd }}$ principal stress) at the bottom of the spacer grooves would change in reflectors with HEU and LEU cores at 8, 9 and 10 years of operation. Their interpretation with respect to the UCS of beryllium is not straightforward because the degradation of UCS for irradiated beryllium is not well characterized in the literature (as presented in Section 3.3.4). The two major sources of data, ATR and SM, provide quite different descriptions of the degradation of the UCS. It is unclear at this point which one of them is more applicable to the MURR reflector case. Nevertheless, the fact that the current reflectors are not showing signs of failure due to high compressive stresses in the grooves indicates that these stresses are not of primary concern. Additionally, the other two stress components at the location of maximum compressive stress are near zero. For that reason, no crack propagation can originate from that location. 
Table 5.3. Compressive stresses in reflector without beamport hole, with HEU and LEU cores, at 8, 10, and 12 years of hypothetical use (units in MPa)

\begin{tabular}{|c|c|c|c|}
\hline & 8 years & 10 years & 12 years \\
\cline { 2 - 4 } & $3^{\text {rd }}$ principal stress & 3 rd principal stress & 3 $3^{\text {rd }}$ principal stress \\
\hline HEU & $-1,079$ & $-1,341$ & $-1,602$ \\
\hline LEU & $-1,171$ & $-1,459$ & $-1,747$ \\
\hline
\end{tabular}

\subsection{Stress Intensity in Beryllium Reflectors and Critical Crack Length}

It is known that beryllium material is particularly susceptible to surface damage during machining. Frequently, the damage takes the form of twins, disturbed surface layers and actual cracks [29]. The physical damage depth may vary substantially; it is affected by the machining operation and the condition of the tools. According to information provided by Materion [29], the damaged layer usually does not exceed 0.002 in. $(0.015 \mathrm{~mm})$ in depth, but may reach $0.008-0.010$ in. $(0.203-0.254 \mathrm{~mm})$ for severe cases, which may drastically affect the fracture strength. After machining is performed, a chemical etching process is used to remove an outer layer 0.002-0.004 in. (0.051-0.102 mm) thick, or thermal treatment is used to anneal out the disturbed layers. This information indicates that a very broad range of surface damage level can be present in the machined beryllium.

To assess the life of historical beryllium reflectors at MURR, an assumption has to be made regarding the quality of the reflector surface. It is quite possible that the quality of the surface of the reflector that failed in 1981 was substantially worse than that of the reflectors that have been installed since. The early grades of beryllium used powder that was obtained via attrition grinding, producing flakeshaped particles [20]. This method of production, as well as the use of recycled beryllium in the process, resulted in a higher spread (standard deviation) in the strength [25]. Additionally, machining of the beamport hole and the edges around it may have introduced cracks and residual stresses that were not fully removed by the etching and annealing. However, this is only a speculation, and it cannot be confirmed almost 40 years after the failure occurred.

The critical crack lengths that are admissible in a structure under specific loading conditions are calculated with the use of an equation binding fracture toughness of the material to the stresses present near the crack (see Section 3.5 for an explanation of this formula):

$$
c=\frac{\left(\frac{K_{I c}}{1.1 \cdot \sigma}\right)^{2}}{\pi}
$$

This equation requires an assumption regarding the degraded fracture toughness of the material. As mentioned in Section 3.3.5, there are no data in the literature describing the fracture toughness of irradiated S-200-F beryllium at the damage level of 5 dpa (equivalent to the damage on the outer surface of the reflector), let alone at the damage level of 17.3 dpa observed on the inner surface of the reflector with the LEU core. Consequently, an assumption has to be made regarding the values of fracture toughness, using the fit to the limited data presented in Figure 3.34. On the basis of equation (5-1) and these multiple assumptions (machined surface condition, degraded fracture toughness), critical crack lengths have been calculated for 8,10 and 12 years of hypothetical operation for the reflectors with HEU and LEU cores, and are presented in Figure 5.10. 


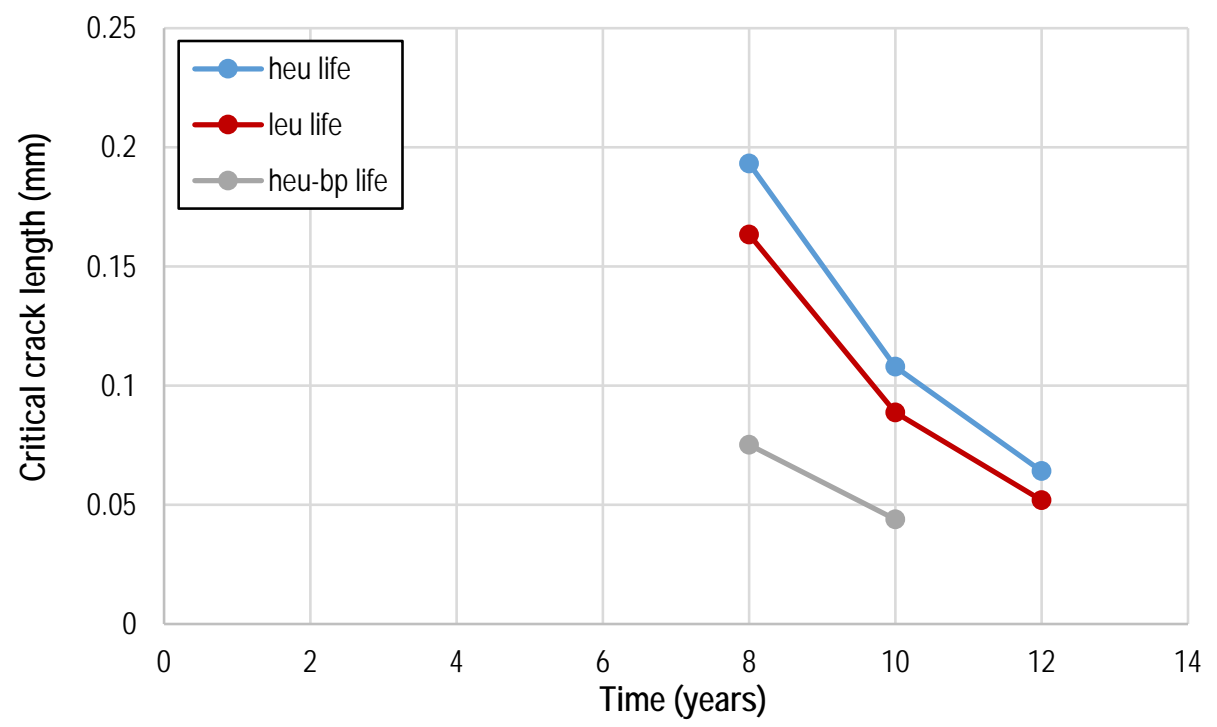

Figure 5.10. Critical crack length in beryllium reflectors at 8, 10, and 12 years of operation with HEU and LEU cores

To assess the life of a structure, the critical crack length for a specific load and fracture toughness is compared to the cracks or imperfections on the surface of that structure. With time (irradiation), the fracture toughness of the beryllium reflector decreases in a similar fashion to UTS degradation, and the stresses due to swelling (and thermal-conductivity degradation) increase. Consequently, the calculated critical crack length decreases.

The assumption of existing crack length (which can take the form of surface roughness) is highly debatable, as the actual data from MURR reflector inspections are not available. The allowable crack size described by Materion is up to $0.254 \mathrm{~mm}$ (see discussion above). If one assumes that the chemical etching removes some of the crack depth, then $0.154 \mathrm{~mm}$ is allowable. This value can be compared with the critical crack lengths presented in Figure 5.10. It can be noted that the critical crack length for the case of the reflector with beamport holes at 8 years of operation was about $0.075 \mathrm{~mm}$. Thus, if the sizes of actual surface imperfections were on the order of maximum allowable crack sizes $[0.154$ $\mathrm{mm}$ ), the failure would occur sooner than that. For the case of the beryllium reflector without beamport holes and with the HEU core, at 8 years of operation the critical crack length is predicted to be about $0.193 \mathrm{~mm}$. For the case of the LEU core, at 8 years of operation the critical crack length is predicted to be about $0.163 \mathrm{~mm}$. These two values are higher than the maximum allowable 0.154 mm crack length.

It is not certain what size of surface imperfections can be expected in the current reflectors without beamport holes. It is certain, however, that the removal of the beamport holes from the design not only reduced the stresses but removed the necessity of elaborate machining of the surface of the reflector, which leads to physical damage of the beryllium structure. Therefore, it can be assumed that the surface condition of the current reflectors is of higher quality than the surface of the historical reflectors with beamport holes.

This approach relies on more uncertain assumptions than the approach based on the maximum stress theory presented in the previous sections. Confidence in using this approach can be augmented when additional information about the surface quality of the reflectors and the data on fracture toughness of highly irradiated beryllium grade S-200-FH become available. Moreover, the simple equation 
presented above to calculate the critical crack length has a quadratic form and is highly sensitive to the changes in the fracture toughness and stresses. Additionally, it was developed for metal sheets (2D cases), not solid bodies (3D state of stress near the crack tip). The crack propagation theories for 3D solid bodies are still under development (see examples in [94]). For these reasons, for beryllium reflector life assessment, this method should be used with caution. 


\section{Conclusions}

The literature review, neutronics calculations, and structural analysis led to the following findings:

1. Despite the increase of core power from $10 \mathrm{MW}$ to $12 \mathrm{MW}$ following conversion from HEU to LEU, the reflector heating decreases by $13.4 \%$ for the LEU core as compared to the HEU core.

2. At the same time, the transmutation-gas production will increase following conversion. The most prevalent component, He-4, will be produced at a rate $9 \%$ higher with the LEU core as compared to the HEU core because of the power uprate. This increase will cause an increase in swelling-induced stresses in beryllium material.

3. The damage, or dpa, for the case of the LEU core will progress at a $10 \%$ faster rate in the most exposed location, which is on the inner face of the reflector. This damage will contribute to a faster change in material properties for the case of the LEU core.

4. The reflector that failed in 1981 was built from a different beryllium grade than the currently manufactured MURR beryllium reflectors (N-200-A versus S-200-FH).

5. The minimum guaranteed UTS of fresh N-200-A beryllium in tension was substantially lower than the guaranteed strength of the current S-200-FH grade used currently at MURR (276 MPa vs $414 \mathrm{MPa}$ ). The method of production of the S-200-FH (HIP) grade also leads to a more uniform, more isotropic and more ductile beryllium than the $\mathrm{N}-200-\mathrm{A}$ beryllium.

6. The degradation rate of these properties due to irradiation may be different for these two grades, but there is no available information on that process in the literature. It is very likely that the reflector materials measured at the ATR were made of Materion's N- or early S-200 grades, so the data from ATR may adequately describe the strength of early MURR reflectors, too.

7. Only a single study reports the results of strength testing after irradiation of the grade of beryllium (S-200-FH) that is currently used at MURR. However, the level of irradiation during these tests was relatively low and the spread in the data is quite substantial. Thus, assumptions have to be made regarding material strength degradation in the current analysis. These strength degradation models may have high uncertainty associated with them; however, the conclusions on lifetime with a HEU core compared to a LEU core would be expected to remain consistent despite the uncertainty levels found, as both fuel types would be similarly impacted.

8. Reduction of thermal conductivity and fracture toughness in irradiated beryllium appears to be less dependent on the grade of beryllium as compared to the UTS and UCS degradations, which appear to be significantly dependent on the grade. Swelling of beryllium at low temperatures $\left(<300^{\circ} \mathrm{C}\right)$ appears to be independent of the grade as well.

9. From the structural analysis performed in this work, it is concluded that the MURR beryllium reflector failure in 1981 was likely due to the tensile stresses on the outer surface of the reflector, especially near the beamport hole edges. Analysis of this reflector predicted that in 9 areas on the outer surface, the stresses exceeded or were very close to the UTS limits of the material ( 4 near the beamport holes and 5 behind the grooves at mid-height). And the corresponding critical crack-length is estimated to be smaller than the allowable flaw size in 
fresh beryllium. Therefore, these stresses are likely to have been responsible for the split of the reflector into upper and lower parts, as observed in 1981.

10. The life of the beryllium reflector with the LEU core will be slightly shorter than the life of the beryllium reflector without a beamport hole operating with the HEU core. The stresses due to heating will be lower for the LEU core, but the stresses due to swelling contribute more to the overall state of stress. Additionally, higher damage due to irradiation with the LEU fuel and the power uprate will more rapidly reduce the strength of the material for this case. Nevertheless, the life of the beryllium reflector with the LEU core will certainly be longer than the life of the reflector that failed in 1981. Thus, the LEU reflector life will be longer than the current HEU replacement schedule.

11. Compressive stresses on the bottom of the grooves will be slightly larger for the LEU core than for both reflector geometries (with and without a beamport hole) with the HEU core. These large compressive stresses are not of great concern. These stresses substantially decrease within a fraction of an inch from the groove. In addition, compressive stresses do not lead to crack propagation in the way that the tensile stresses do. Cracking on the inner surface of the grooves is possible, on the basis of this analysis.

12. Because the strength degradation data are uncertain, it cannot be determined precisely if and by how many years the replacement schedule for the reflector can be extended beyond the current practice of 8 years of operation. A hypothetical study of stress levels beyond the 8-year limit indicates that some margin to failure does exist for the current replacement schedule, but because of the uncertainties, no extension of this schedule is recommended on the basis of this study.

13. For more precise life estimation, higher-fidelity data regarding the degradation of UTS and fracture toughness of the S-200-FH grade is required. It may be examined through experimental testing under irradiation conditions relevant for the MURR beryllium reflector. It is also necessary to better understand the distribution of intrinsic and surface flaws within the fabricated reflector.

14. Many assumptions are made in the analysis presented here. Some of them may be overly conservative, while others may be less conservative or not at all conservative. However, each of these assumptions influences the results of all analyzed cases in the same fashion. Thus, if the stresses due to swelling progress at a faster rate than assumed in the analysis, all three analyzed models will be affected similarly. As a result, the relative life of beryllium reflectors should remain in the same order, with the life of the beryllium reflector with the beamport hole and the HEU core < the life of the beryllium reflector without the beamport hole and with the LEU core $<$ the life of the beryllium reflector without the beamport hole and with the HEU core.

15. The lifetimes of beryllium reflectors with both the HEU and LEU core were analyzed to exceed the current operational limit of 26,000 MWd achieved at 8 years of operations. 


\section{Acknowledgment}

This work was sponsored by the U.S. Department of Energy, Office of Material Management and Minimization in the U.S. National Nuclear Security Administration Office of Defense Nuclear Nonproliferation under Contract DE-AC02-06CH11357. 


\section{References}

[1] N. Peters, J. McKibben and L. Foyto, "The Impact of the HEU to LEU Fuel Conversion on the Lifetime and Efficacy of Beryllium: The Primary Neutron Reflector at High-performance Research and Test Reactors," in Test Research and Training Reactor, Columbia, M0, 2013.

[2] University of Missouri Research Reactor (MURR), Specification Drawing Beryllium Reflector, Drawing No. 193, Columbia, MO, 1981.

[3] University of Missouri Research Reactor (MURR), Specification Drawing Beryllium Reflector, Drawing No. 193, Columbia, MO, 2001.

[4] J. Stillman, E. Feldman, L. Foyto, K. Kutikkad, J. McKibben, N. Peters and J. Stevens, "Technical Basis in Support of the Conversion of the University of Missouri Research Reactor (MURR) Core from Highly-Enriched to Low-Enriched Uranium - Core Neutron Physics, ANL/RERTR/TM-1230," Argonne National Laboratory, Lemont, IL, 2012.

[5] J. A. Stillman, E. Feldman, D. Jaluvka, C. Bojanowski, A. Tentner, T. Heltemes, B. Dionne, M. Kalimullah, J. Stevens, E. Wilson, W. Cowherd, B. Rickman, C. McKibben, K. Kutikkad, N. Peters and L. Foyto, "Low-Enriched Uranium Conversion Preliminary Safety Analysis Report for the University of Missouri Research Reactor," University of Missouri Research Reactor, 2017.

[6] J. Saddler, N. Peters, J. McKibben and L. Foyto, "Impact of HEU to LEU Fuel Conversion on the Lifetime and Efficacy of Beryllium as the Primary Neutron Reflector at High Performance Research and Test Reactors," in 6th International Symposium on Material Testing Reactors, Bariloche, Rio Negro, Argentina, 2013.

[7] G. Was, Fundamentals of Radiation Materials Science, Springer-Verlag Berlin Heidelberg, 2007.

[8] J. Beeston, "Properties of Irradiated Beryllium Statistical Evaluation, TREE-1063," EG\&G Idaho, Inc., Idaho Falls, ID, 1976.

[9] C. Dorn, K. Tsuchiya, Y. Hatano, P. Charkov, M. Kodama and H. Kawamura, "Status of Material Development for Lifetime Expansion of Beryllium Reflector," in Proceedings of the 4th International Symposium on Material Testing Reactors, Oarai, Japan, 2011.

[10] L. Snead, "Low-temperature low-dose neutron irradiation effects on beryllium," Journal of Nuclear Materials, vol. 326, pp. 114-124, 2004.

[11] S. Morozumi, S. Goto and M. Kinno, "Effects of Distribution of Helim Bubbles on the Tensile Properties and Swelling of Neutron-Irradiated and Annealed Beryllium," Journal of Nuclear Materials, vol. 68, pp. 82-91, 1977.

[12] V. Chakin, V. Kazakov, R. Melder, Y. Goncharenko and I. Kupriyanov, "Effects of neutron irradiation at 70-200 C in beryllium," Journal of Nuclear Materials, Vols. 307-311, pp. 647-652, 2002.

[13] V. Chakin, A. Posevin and R. Latypov, "Radiation damage in beryllium at 70-440 C and neutron fluence (0.3-18) * 10^22 (E>0.1 MeV)," Atomic Energy, vol. 101, pp. 743-749, 2006.

[14] V. Chakin, R. Rolli, H. Schneider, A. Moelsang, P. Kurinskiy and W. Van Renterghem, "Pores and cracks in highly neutron irradiated beryllium," Journal of Nuclear Materials, pp. 3-8, 2011.

[15] L. Sannen, C. De Raedt, F. Moons and Y. Yao, "Helium content and induced swelling of neutron irradiated beryllium," Fusion Engineering and Design, vol. 29, pp. 470-474, 1994.

[16] F. Scaffidi-Argentina, M. Dalle Donne, C. Ferrero and C. Ronchi, "Helium induced swelling and tritium trapping mechanisms in irradiated beryllium: A comprehensive approach," Journal of Fusion Engineering and Design, vol. 27, pp. 275-282, 1995. 
[17] COMSOL, Inc., "Introduction to COMSOL Multiphysics - Version 5.3a," COMSOL, Inc., Burlington, MA, 2017.

[18] X-5 Monte Carlo Team, "MCNP-A General Monte Carlo N-Particle Transport Code, Version 5 Volume I, II and III, LA-UR-03-1987/LA-CP-03-0245/LA-CP-03-0284," Los Alamos National Laboratory, Los Alamos, NM, 2003.

[19] D. Ilas, "Impact of HFIR LEU Conversion on Beryllium Reflector Degradation Factors, ORNL/TM2013/441," Oak Ridge National Laboratory, Oak Ridge, TN, 2013.

[20] K. Walsh, Beryllium Chemistry and Processing, Material Park, OH: ASM International, 2009.

[21] K. Walsh, ASM Handbook, Beryllium and Beryllium Alloys, Materials Park, OH: ASM International, 2010.

[22] D. Floyd and J. Lowe, Beryllium Science and Technology, Volume 2, Springer US, 1979.

[23] C. L. Haertling, "Beryllium (Be) Handbook, LA-UR-20-23733," Los Alamos National Laboratory, Los Alamos, NM, 2020.

[24] E. Simonds and D. Keller, "Reactor Materials Quarterly Technical Progress Review," The University of Michigan, 1967.

[25] Materion, "Thermomechanical Properties of Beryllium, Materion Data Sheet TR-1182," Materion Corporation, 1995.

[26] Materion, "Materion - Products, Uses \& Descriptions," 2020b. [Online]. Available: https://materion.com/-/media/files/beryllium/engineering-design/materion-product-usesand-descriptions.pdf.

[27] Materion Inc., "HIP Beryllium Achieves Full Commercial Status," 2020c. [Online]. Available: https://materion.com/-/media/files/beryllium/albemetmaterials/hipberylliumachievesfullcommercialstatus.pdf. [Accessed 2002 2020].

[28] Materion Inc., "A Guide to Deisgn and Fabrication with Beryllium," Materion Inc..

[29] Materion, "Materion - Designing and Fabricating with Beryllium," 2020a. [Online]. Available: https://materion.com/-/media/files/beryllium/beryllium-materials/mb001designingandfabricatingberyllium.pdf.

[30] Materion, "S-200-FH Grade Beryllium Spec Sheet," Materion, 2012.

[31] Materion, "S-200-F Standard Grade beryllium Spec Sheet," Materion, 2014.

[32] The Brush Beryllium Company, "QMV Beryllium Specification Sheet," Brush Beryllium Company, Cleveland, OH, 1961.

[33] V. Kuksenko, S. Roberts and E. Tarleton, "The hardness and modulus of polycrystalline beryllium from nanoindentation," International Journal of Plasticity, vol. 116, pp. 62-80, 2019.

[34] Swales Aerospace, "Elastic properties measurements for beryllium alloys," Swales Aerospace, 2005.

[35] A. Goldberg, "Atomic, Crystal, Elastic, Thermal, Nuclear, and Other Properties of Beryllium, UCRL-TR-224850," Lawrence Livermore National Laboratory, Livermore, Ca, 2006.

[36] W. Hodge, "Beryllium for Structural Applications - A Review of the Unclassified Literature, 1958-1960, DMIC Report 168," Batelle Memorial Institute, Columbus, OH, 1962.

[37] Kirk-Othmenr, Encyclopedia of Chemical Technology, I. John Wiley \& Sons, Ed., 2000.

[38] C. H. Wu, J. Bonal, H. Kwast, F. Moons, G. Pott, H. Werle and G. Vieider, "EU results on neutron effects on PFC materials," Fusion Engineering and Design, Vols. 39-40, pp. 263-273, 1998.

[39] Materion, Personal correspondence with Keith J. Smith VP Nuclear, Science \& Government Affairs at Materion, 2020. 
[40] Materion, Inc., "Characterization of Beryllium Structural Grade, TM-778 / S-200F," Materion, Inc., 1985.

[41] J. Beeston, "Gas Release and Compression Properties in Beryllium Irradiated at 600 and 750C, IN-1057," Idacho Nuclear Corporation, Idaho Falls, ID, 1967.

[42] F. Moons, R. Chaouadi and J. Puzzolante, "Fracture behaviour of neutron irradiated beryllium," Fusion Engineering and Design, pp. 187-193, 1998.

[43] F. Grensing, D. Hashiguchi and J. Marder, "Fracture Toughness of Vacuum Hot Pressed Beryllium Powder," in Advances in Powder Metallurgy, vol. 2, Princeton, N.J., 1990, pp. 27-36.

[44] C. Swenson, "HIP beryllium: Thermal expansivity from 4 to $300 \mathrm{~K}$ and heat capacity from 1 to 108 K," Journal of Applied Physics, vol. 70, pp. 3046-3051, 1991.

[45] K. Ammigan, P. Hurh, R. Zwaska, S. Bidhar, K. Yonehara, K. Anderson, G. Waver, M. Calviani, C. Losito, C. Torregrosa, R. Seidenbinder, K. Kershaw, M. Butcher, V. Kuksenko, D. Senor, A. Casella, C. Densham, A. Atherton, G. Burton, O. Caretta, T. Davenne, P. Loveridge, J. O'Dell, M. Fitton, T. Ishida, S. Makimura, E. Wakai and S. Roberts, "Results and experience from the BeGrid experiment (HRMT24) and an overview of the follow-up BeGrid2 experiment (HRMT43)," in HiRadMat User Day, 2018.

[46] F. Scaffidi-Argentina, G. Longhurst, V. Shestakov and H. Kawamura, "Beryllium R\&D for fusion applications," Fusion Engineering and Design, Vols. 51-52, pp. 23-41, 2000.

[47] F. Moons, L. Sannen, A. Rahn and J. Van De Velde, "Neutron irradiated beryllium: tensile strength and swelling," Journal of Nuclear Materials, vol. 41, pp. 823-827, 1996.

[48] Dynatech, "Thermal conductivity of hot pressed beryllium between 4 and $600 \mathrm{~K}, \mathrm{TEX}-6$," November 1976. [Online].

[49] V. Chakin, R. Latypov, D. Suslov and I. Kupriyanov, "Thermal Conductivity of Beryllium under Low Temperature High Dose Neutron Irradiation," in Proceedings of the Sixth IEA International Workshop on Beryllium Technology for Fusion, Miyazaki City, Japan, 2004.

[50] D. Lemon and W. Brown, "Fracture Toughness of Hot-Pressed Beryllium," in 24th AIAA/ASME/ASCE/AHS Structures, Structural Dynamics and Materials Conference, Lake Tahoe, NV, 1983.

[51] L. Barker and A. Jones, "Fracture toughness of CIP-HIP beryllium at elevated temperatures, MTL TR 86-10," U.S. Army Material Technology Laboratory, Watertown, MA, 1986.

[52] D. Gelles, G. Sernayev, M. Dalle Donne and H. Kawamura, "Radiation effects in beryllium used for plasma protection," Journal of Nuclear Materials, Vols. 212-215, pp. 29-38, 1994.

[53] V. Barabash, S. Tanaka and R. Matera, "Beryllium Assessment and Recommendation for Application in ITER Plasma Facing Components," in IEA International Workshop on Beryllium Technology for Fusion, Mito City, Japan, 1997.

[54] V. Barabash, G. Federici, M. Rodig, L. Snead and C. Wu, "Neutron irradiation effects on plasma facing materials," Journal of Nuclear Materials, pp. 138-146, 2000.

[55] V. Chakin, J. Reimann, A. Moeslang, R. Latypov and A. Obukhov, "Thermal conductivity of highly neutron-irradiated beryllium in nuclear fusion reactors," Progress in Nuclear Energy, vol. 57, pp. 2-7, 2012.

[56] V. Chakin, V. Kazakov, A. Teykovtsev, V. Pimenov, G. Shimansky, Z. Ostrovsky, D. Suslov, R. Latypov, S. Belozerov and I. Kupriyanov, "High dose neutron irradiation damage in beryllium as blanket material," Fusion Engineering and Design, Vols. 58-59, pp. 535-541, 2001.

[57] V. Chakin and Z. Y. Ostrovsky, "Evolution of beryllium microstructure under high-dose neutron irradiation," Journal of Nuclear Materials, Vols. 307-311, pp. 657-663, 2002. 
[58] V. Chakin, A. Posevin and I. Kupriyanov, "Swelling, mechanical properties and microstructure of beryllium irradiated at $200 \mathrm{C}$ up to extremely high neutron doses," Journal of Nuclear Materials, Vols. 367-370, pp. 1377-1381, 2007.

[59] V. Chakin, R. Melder and S. Belozerov, "Experience of Beryllium Blocks Operation in the SM and MIR Nuclear Reactors Useful for Fusion," in Proceedings of the Sixth IEA International Workshop on Beryllium Technology for Fusion, Miyazaki City, Japan, 2004 a.

[60] D. Sysov, V. Chakin and R. Latypov, "Influence of high dose neutron irradiation on thermal conductivity of beryllium," Journal of Nuclear Materials, Vols. 307-311, pp. 664-667, 2002.

[61] F. Scaffidi-Argentina, M. Donne, C. Ronchi and C. Ferrero, "ANFIBE: A Comprehensive Model for Swelling and Tritium Release from Neutron-Irradiated Beryllium-II: Comparison of Model Predictions with Experimental Results," Journal of Fusion Technology, vol. 33, pp. 146-163, 1998.

[62] R. Chaouadi, A. Leenaers, J. Puzzolante and M. Scibetta, "Radiation Effects on the Mechanical Properties of Beryllium," in Proceedings of the 4th IEA International Workshop on Beryllium Technology for Fusion, Kalsruhe, Germany, 1999.

[63] R. Chaouadi, F. Moons and J. Puzzolante, "Tensile and Fracture Toughness Test Results of Neutron Irradiated Beryllium, TEC97/51.F040010/15 /RC-Rev.1," SCK•CEN, Mol, Belgium, 1997.

[64] J. Beeston, L. Miller, E. Wood and R. Moir, "Comparison of Compression Properties and Swelling of Beryllium Irradiated at Various Temperatures," Journal of Nuclear Materials, vol. 122 \& 123, pp. 802-809, 1984.

[65] L. Miller, J. Beeston, B. Harris and C. Wong, "Special Topics Reports for the Reference Tandem Mirror Fusion Breeder: Beryllium Lifetime Assessment," EG\&G Idaho, Inc., Idaho Falls, ID, 1984.

[66] T. McCarville, D. Berwald, W. Wolfer, F. Fulton, J. Lee, R. Maninger, R. Moir, J. Beeston and L. Miller, "Technical Issues for Beryllium Use in Fusion Blanket Applications, UCID-20319," Lawrence Livermore National Laboratory, 1985.

[67] D. Harries, M. Dalle Donne and F. Scaffidi-Argentina, "Beryllium Irradiation Embrittlement Test (BSBE) Programme, Assessment of the Tensile and Fracture Toughness Test Data FZKA 6376," Forschungszentrum Karlsruhe, Karlsruhe, Germany, 2000.

[68] J. Beeston, "Beryllium Metal as a Neutron Moderator and Reflector Material," Nuclear Engineering and Design, vol. 14, pp. 445-474, 1970.

[69] B. Winkel, "Stress and Deformation Analysis of Irradiation Induced Swelling," in Pressure Vessels and Piping - Material - Nuclear Conference, Miami Beach, FL, 1974.

[70] P. Chakin, I. Kupryanov and R. Melder, "State of beryllium after irradiation at low temperature up to extremely high neutron doses," pp. 1347-1352, 2004c.

[71] I. Kupriyanov, V. Gorokhov, R. Melder, Z. Ostrovsky and G. Nikolaev, "Neutron Irradiation Behavior of ITER Candidate Beryllium Grades," in Proceedings of the 3rd IEA International Workshop on Be Technology for Fusion, Mito City, Japan, 1997.

[72] K. Wikle, "Metallurgical Process Development Division Brush Beryllium Company," in Semiannual meeting of the American Society of Tool Engineers, Los Angeles, CA, 1958.

[73] Case Western Reserve University, "Encyclopedia of Cleveland History," Case Western Reserve University, 2020. [Online]. Available: https://case.edu/ech/articles/b/brush-wellman-inc. [Accessed 0305 2020].

[74] I. Kupriyanov, V. Gorokhov, G. Nikolaev and V. Burmistrov, "Research and development of radiation resistant beryllium grades for nuclear fusion applications," Journal of Nuclear Materials, Vols. 233-237, part 2, pp. 886-890, 1996. 
[75] V. Chakin, "Evolution of the microstructure and physical-mechanical properties of beryllium under high neutrons exposure (in Russian), PhD dissertation," Ulyanovsk State University, Ulyanovsk, Russia, 2017.

[76] J. Beeston, "Fracture Toughness of Irradiated Beryllium," EG\&G Idaho, Inc, Idaho Falls, ID, 1978.

[77] F. Moons, R. Chaouadi and J. Puzzolante, "Fracture behaviour of neutron irradiated beryllium," Fusion Engineering and Design, pp. 187-193, 1998.

[78] M. Billone, M. Dalle Donne and R. Macaulay-Newcombe, "Status of beryllium development for fusion applications," Fusion Engineering and Design, vol. 27, pp. 179-190, 1995.

[79] NRC, "Guidelines for Preparing and Reviewing Applications for the Licensing of Non-Power Reactors, Format and Content, NUREG-1537, part 1," United States Nuclear Regulatory Commission, 1996.

[80] NRC, "Guidelines for Preparing and Reviewing Applications for the Licensing of Non-Power Reactors, Standard Review Plan and Acceptance Criteria, NUREG-1537, part 2," United States Nuclear Regulatory Commission, 1996.

[81] M. Belal and W. Styrdom, "The Status of the Beryllium Reflector in the Safari-1 Research Reactor," in Proceedings of an International Conference organized by the International Atomic Energy Agency (IAEA), Rabat, Morocco, 2011.

[82] L. Moloko, "Impact of beryllium reflector ageingon Safari-1 Reactor core parameters," NorthWest University, Potchefstroom, South Africa, 2011.

[83] J. Ahlf and W. Zijp, "Upgrading Activities for the HFR Petten," in Internatioanl Symposium on Research Reactor Safety, Operations and Modifications, Chalk River, Ontario, Canada, 1989.

[84] J. Durney and D. Croucher, "Replacement of Core Components in the Advanced Test Reactor, IAEA-SM-310/21," in International Symposium on Research Reactor Safety, Operations and Modifications, Chalk River, Ontario, Canada, 1990.

[85] E. Koonen, "Experience Gained from the BR2 Beryllium Matrix Replacement and Second Matrix Surveillance Programme," in International Symposium on Research Reactor Safety, Operations and Modifications, Chalk River, Ontario, Canada, 1990.

[86] R. Budynas and J. Nisbett, Shigley's Mechanical Engineering Design, 9th edition, New York: McGraw-Hill, 2011.

[87] P. Hui, "A Survey of Beryllium Technology and Nuclear Applications, ORNL-4421," Oak Ridge National Laboratory, Oak Ridge, TN, 1969.

[88] T. Tomberlin, "Beryllium - AUnique Material in Nuclear Applications, INEEL/CON-04-01869," Idaho National Engineering and Environmental Laboratory, Idaho Falls, ID, 2004.

[89] R. Christensen, "A Two-Property Yield, Failure (fracture) Criterion for Homogenosu, Isotropic Materials," Journal of Engineering Materials and Technology, vol. 129, pp. 45-52, 2004.

[90] R. Christensen, "A Comprehensive Theory of Yielding and Failure for Isotropic Materials," Journal of Engineering Materials and Technology, pp. 173-181, 2007.

[91] R. Christensen, "Evaluation of Ductil/Brittle Failure theory and Derivation of the Ductile/Brittle Transition Temperature," ASME Journal of Applied Mechanics, vol. 83, pp. 1-10, 2016.

[92] NGK Berylco, "About NGK Metals Corporation," 2020. [Online]. Available: https://www.ngkmetals.com/index.cfm/m/3/About. [Accessed 3108 2020].

[93] J. Henker, I. Spiker and W. Castner, "The mechanical behavior of cross-rolled beryllium sheet; NASA-TM-4397," NASA, 1992. 
[94] J. Wang, L. Ren, L. Xie, H. Xie and T. Ai, "Maximum mean principal stress criterion for threedimensional brittle fracture," International Journal of Solids and Structures, vol. 102, pp. 142154, 2016.

[95] Internuclear Company, "Stress and Thermal Analysis of the Beryllium Reflector for the University of Missouri Reactor, TM-ERS-62-1," 1962b.

[96] Internuclear Company, "Missouri University Research Reactor Design Data, Volume I," Clayton, MO, 1962.

[97] COMSOL Inc., Comsol Multiphysics Reference Manual v5.3a, 2019.

[98] N. Peters, "Spatial Distribution of the Effective Fission Energy Deposition within the MURR Core: HEU vs. LEU Fuel Comparison, TDR-0140," University of Missouri Research Reactor, 2015.

[99] I. Charak, "Thermal and Hydraulic Design for Reactor Regions Cooled by Pool Water, MURR Design Data, TM-IC-62-5:," Internuclear Company, 1962. 


\section{APPENDIX A: MURR Beryllium Reflector Flux, Gas Production, Damage, and Heating}

\section{A.1 Introduction}

MURR is a compact core operating at a high power density. A cross-sectional view of the reactor, showing the eight elements of the core (numbered 1 through 8), reactor control system, and experimental facilities, is provided in Figure A.1. In order to mitigate neutron losses from leakage and sustain the neutron economy, the core is surrounded by an annular beryllium reflector. Neutron and photon fluence integrated over time cause internal stresses and damage (dpa) in the beryllium which necessitates routine replacement (currently replaced after $\sim 8$ years typical operation) in order to maintain the integrity of the reflector.

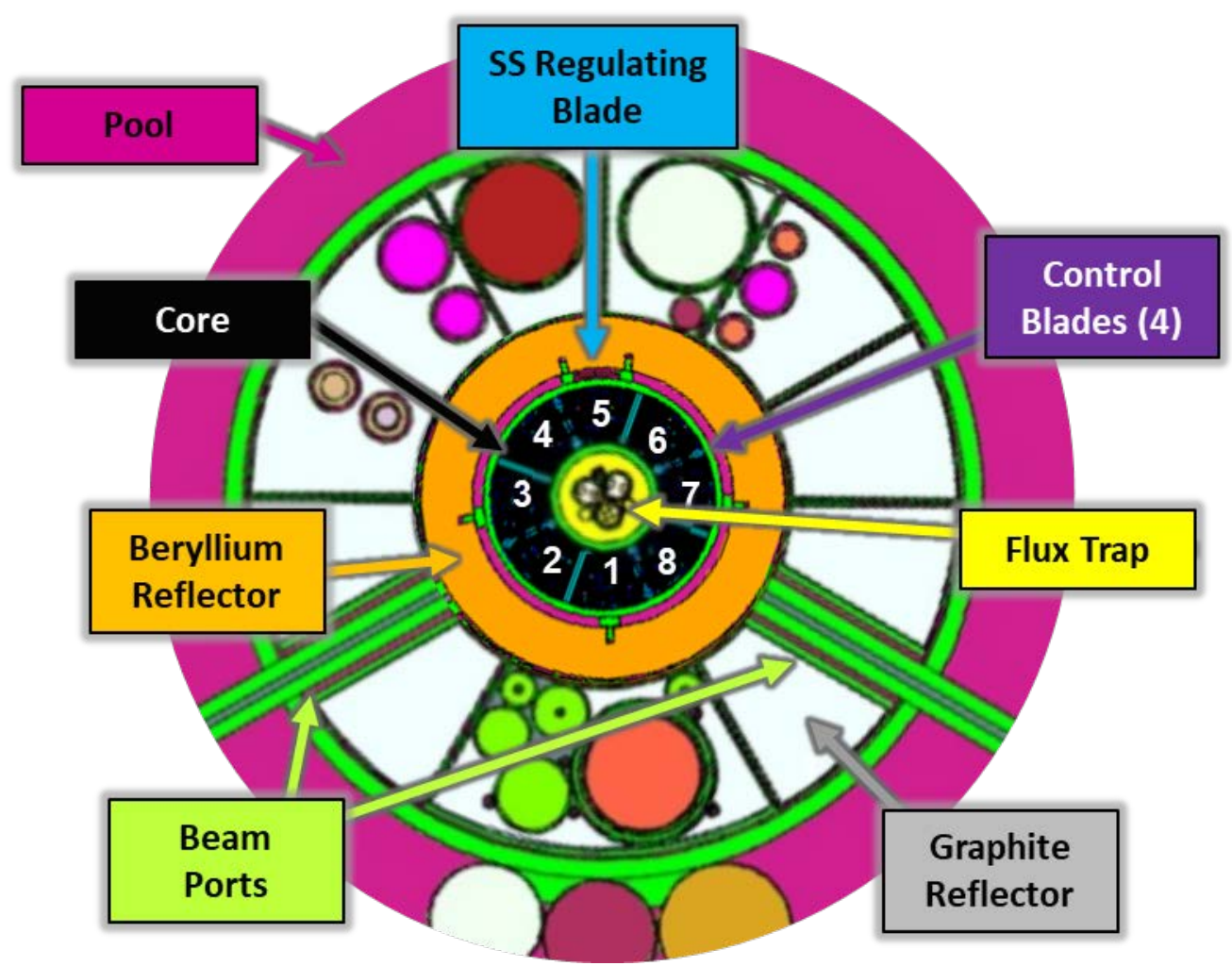

Figure A.1. Cross-sectional view of MURR layout

In order to support assessment of the MURR beryllium reflector lifetime, calculations were completed to predict the neutron flux, He-4 gas production, and neutron damage over an anticipated life of the reflector. Neutron and photon heating in the reflector were also calculated. Predictions of the reflector irradiation conditions were made using the software MCNP5 v. 1.6 [18]. The MCNP5 models used in the current analysis are the same as those used to support safety analyses for HEU and LEU cores [5], but with the beryllium reflector discretized into many more regions to provide the data needed for this work.

The chemical composition of the reflector material in the MCNP5 model was based on a material assay of the reflector and recommended by the reactor operator [4]. MCNP5 does not model material 
depletion. Rather, the neutron flux, He-4 gas production rate, damage rate, and neutron and photon heating rates were predicted based on the conditions of a fresh (unirradiated) beryllium reflector. Thus, the analysis neglects how any changes in the reflector composition over its lifetime might affect the flux level, reaction rates, or heating in the reflector. This is a reasonable assumption since the weekly core changeout provides a relatively constant neutron and photon flux impinging on the reflector from core leakage. A separate analysis found that the neutron flux remains relatively unchanged with increasing reflector burnup. The magnitude of the fast flux increases by just $0.2 \%$ and the total flux decreased by only $1.6 \%$ over an extreme irradiation history of nearly 14 years.

In this simulation of reflector irradiation conditions with the MURR HEU and LEU cores, the core was maintained at a constant burnup state under equilibrium xenon conditions over the typical multiyear depletion of the reflector. This is reasonable since weekly operating cycles in MURR are relatively fixed in terms of core burnup, and the majority of the cycle is spent at equilibrium xenon conditions with the reactor control blades positioned from 23 to 25 inches withdrawn. Table A.1 summarizes cores which are typical for current MURR operations with HEU and anticipated with LEU following conversion.

Table A.1. MURR core state for prototypic operations with HEU and LEU fuel

\begin{tabular}{|l|l|c|c|}
\hline \multirow{2}{*}{ Fuel } & \multirow{2}{*}{ State } & Core Burnup (MWd) & $\begin{array}{l}\text { Critical Blade Position (inches } \\
\text { withdrawn) }\end{array}$ \\
\hline \multirow{2}{*}{ HEU } & Xenon-free & 579 & 17.8 \\
\cline { 2 - 4 } & Equilibrium Xenon & 599 & 24.0 \\
\hline \multirow{2}{*}{ LEU } & Xenon-free & 687 & 17.6 \\
\cline { 2 - 4 } & Equilibrium Xenon & 711 & 24.3 \\
\hline
\end{tabular}

\section{A.2 Relevant Reactions for Gas Production in Irradiated Beryllium}

The neutron and photon reactions considered most relevant for the analysis of the MURR reflector depletion are:

$$
\begin{array}{ll}
\text { Be- }-9+n \rightarrow \text { Be- } 8+2 n(n, 2 n \text { reaction) } & \text { Be- } 8 \rightarrow 2 \text { He- } 4 \text { (short decay) } \\
\text { Be- } 9+n \rightarrow \text { He- } 6+\text { He- } 4 \text { (n, } \alpha \text { reaction) } & \text { He- } 6 \rightarrow \text { Li- } 6 \text { (short decay) } \\
\text { Be- } 9+\gamma \rightarrow n+2 H e-4 \text { ( } \gamma, 2 \alpha \text { reaction) } & \\
\text { Li }-6+n \rightarrow \text { He- } 4+\text { H-3 (n,T reaction) } &
\end{array}
$$

The majority of the gaseous by-products of beryllium irradiation is He-4. The source of the He-4 is mostly produced by threshold $(n, 2 n)(78 \%)$ and $(n, \alpha)(15 \%)$ reactions in Be-9. A small fraction of the total He-4 (7\%) is produced from the Li-6 (n,T) reaction. There is also a photonuclear reaction for high-energy photons with Be-9 which can produce He-4. In addition to the He-4 production, another $7 \%$ of the total gas concentration in the irradiated reflector is tritium, which is produced primarily through $(\mathrm{n}, \mathrm{T})$ reactions in Li-6.

Results relevant to the MURR reflector lifetime analysis were obtained based on detailed spatial and energy discretizations of the MURR beryllium reflector in MCNP5. The reflector was segmented for tally purposes with 28 radial zones and 45 axial zones. Segmentation details are provided in Appendix $B$. The neutron flux and reaction rates were also tallied with 15 energy bins. The group structure is summarized in Appendix C. 
The following tallies were implemented in MCNP5 models of the HEU and LEU cores:

- Neutron flux

- $\quad(n, 2 \mathrm{n})$ reaction rate in $\mathrm{Be}-9(\mathrm{MT}=16)$

- $(n, \alpha)$ reaction rate in $\mathrm{Be}-9(\mathrm{MT}=107)$

- $(\gamma, 2 \alpha)$ reaction rate ${ }^{1}$ in Be-9 $(\mathrm{MT}=29)$

- $\quad(\mathrm{n}, \mathrm{T})$ reaction rate in $\mathrm{Li}-6^{2}(\mathrm{MT}=105)$

- Beryllium damage from atomic displacement (MT=444)

- Beryllium heating from neutron and photon reactions

All tallies from MCNP5 were normalized to a core power of $10 \mathrm{MW}$ for HEU and $12 \mathrm{MW}$ for LEU. The recoverable energy per fission assumed in the analysis was $198.36 \mathrm{MeV} /$ fission for $\mathrm{HEU}$ and 201.04 MeV/fission for LEU. These recoverable energy/fission values, which include energy released from capture, is from an MCNP5 analysis performed at MURR and reported by Peters in [98].

\section{A.3 Neutron Flux: Beryllium Reflector with HEU Core}

Figure A.2 shows that the neutron flux decreases with penetration into the reflector, and is highest near the core midplane. Figure A.3 shows the radial distribution of the fast flux (E $>1.0 \mathrm{MeV}$ ).

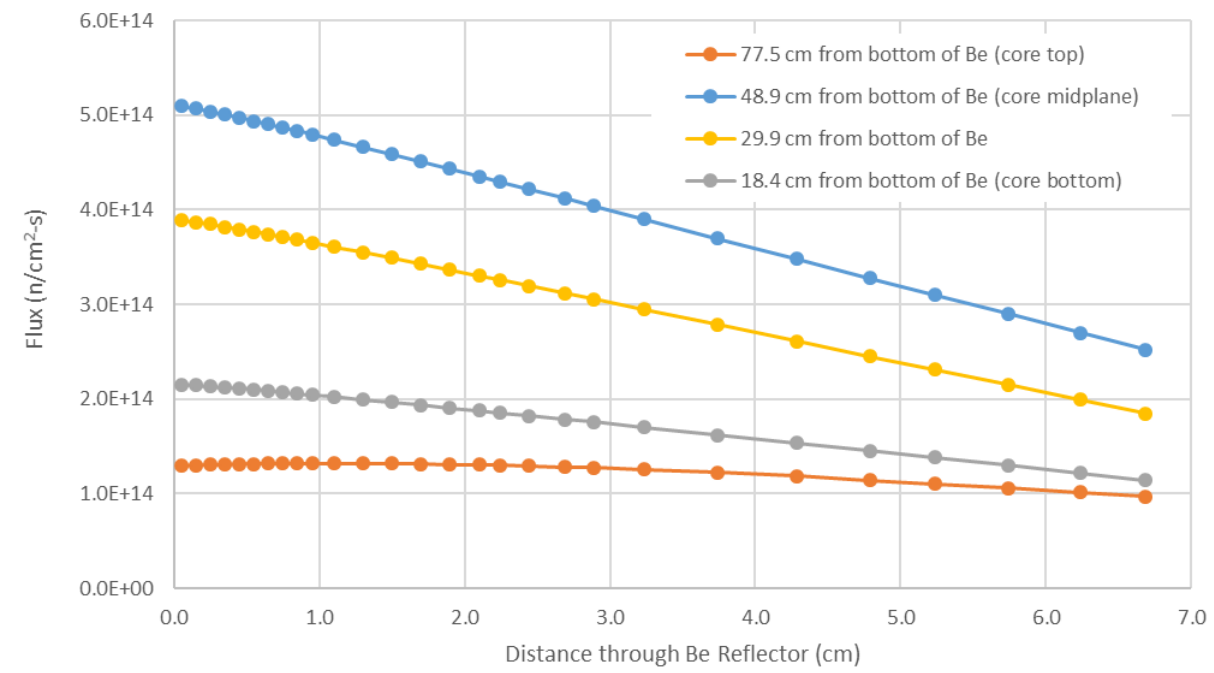

Figure A.2. Total flux in beryllium reflector (HEU core at equilibrium Xe)

${ }^{1}$ Calculated with MCNP6.2. MCNP5 is not capable of processing the $(\gamma, 2 \alpha)$ reaction type.

2 There is no Li- 6 in the fresh beryllium, so a uniform Li- 6 concentration of $\sim 4.5$ appm $(5.6 \mathrm{E}-07$ at/b-cm with a fresh Be-9 atom density of $1.2 \mathrm{E}-01 \mathrm{at} / \mathrm{b}-\mathrm{cm}$ ) was selected for the tally purpose only. This concentration was selected based on an assumed equilibrium concentration of Li- 6 in the reflector, which is reached rather quickly ( $<2$ years). 


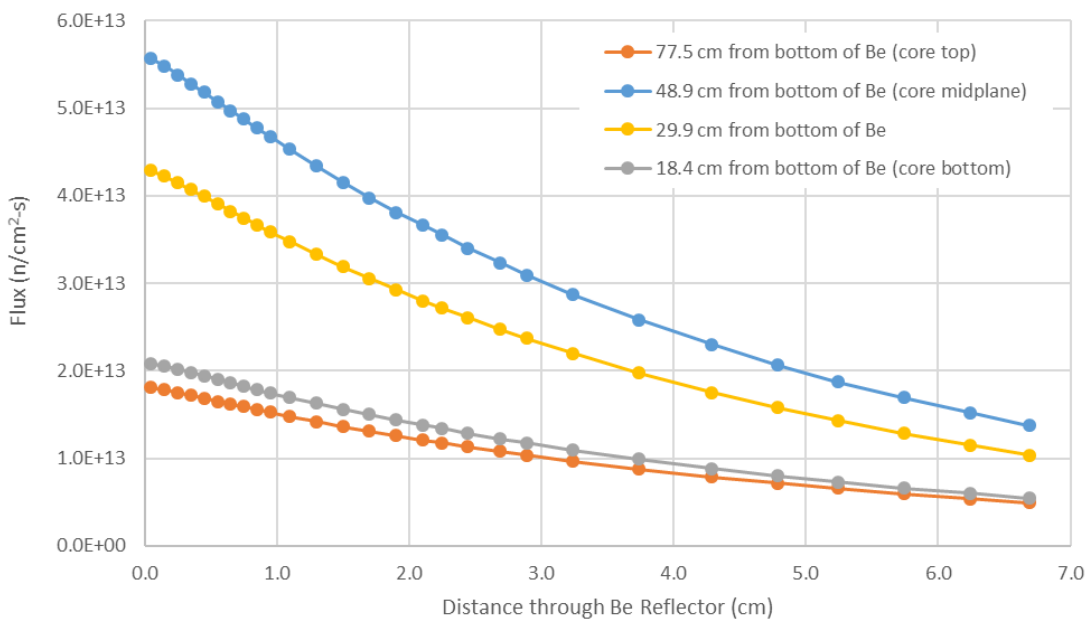

Figure A.3. Flux ( $\mathrm{E}>1.0 \mathrm{MeV}$ ) in beryllium reflector (HEU core at equilibrium Xe)

The three-dimensional contour plots provided in Figure A.4 to Figure A.8 were generated from the MCNP5 results to illustrate the behavior of the neutron flux in the reflector. It should be noted that the figures are not to scale in the radial or axial dimension, as they are plotted based on the spatial bin number in the MCNP5 tallies. Because these bin sizes are not uniform, the flux shape is somewhat visually distorted and does not appear smooth in the radial direction as in Figure A.2 and Figure A.3. This is especially apparent in the perspective shown in Figure A.6. Nonetheless, the shape of the flux can still be discerned, and the results show that the total and fast neutron flux decrease severely with distance from the inner to outer radial surface of the reflector and axially from the core midplane to the top and bottom of the reflector. As can be seen in Figure A.8, the thermal flux also decreases with penetration distance into the reflector, but not as sharply as the fast flux. This is because the neutron flux spectrum becomes increasingly more thermalized from elastic scattering with the beryllium, increasing the fraction of lower energy neutrons with $\mathrm{E}<100 \mathrm{keV}$ from $75 \%$ at the inner surface of the reflector to $86 \%$ at the outermost surface, and more than $90 \%$ at the extreme axial positions.

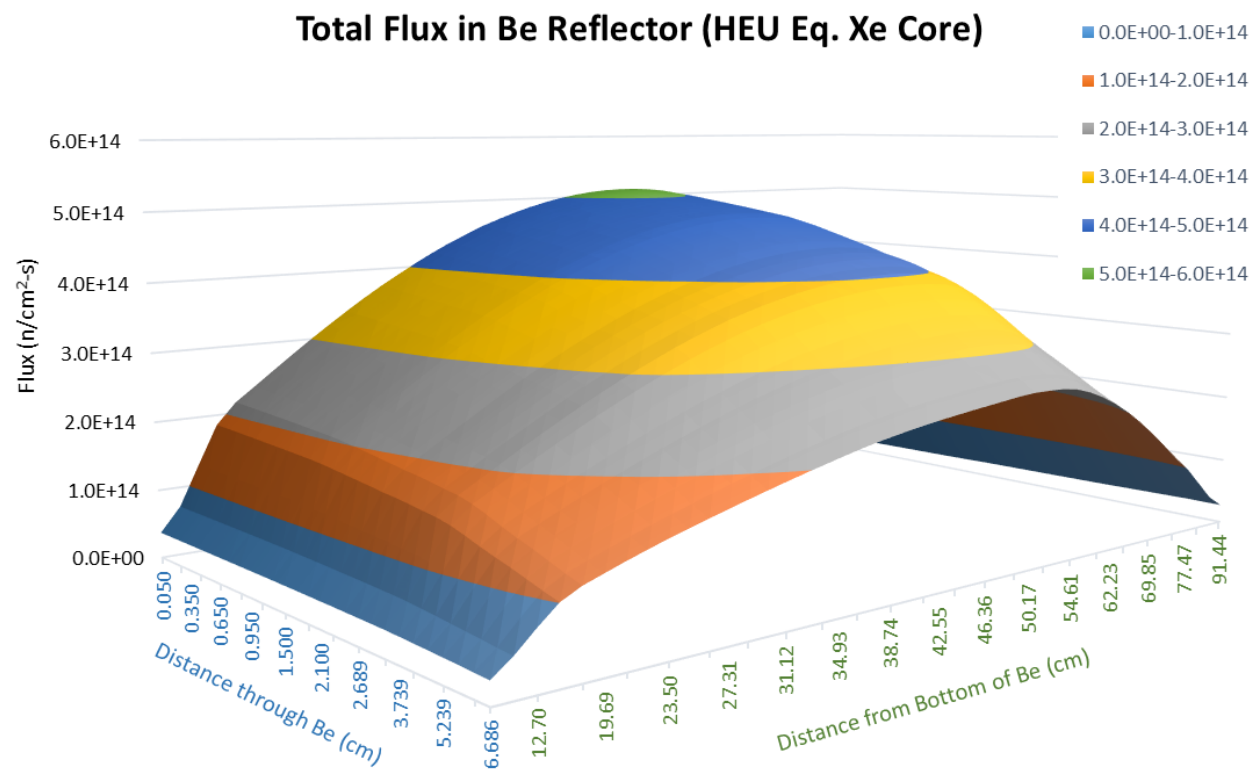

Figure A.4. Total flux in beryllium reflector (HEU core at equilibrium Xe) 
Total Flux in Be Reflector (HEU Eq. Xe Core)

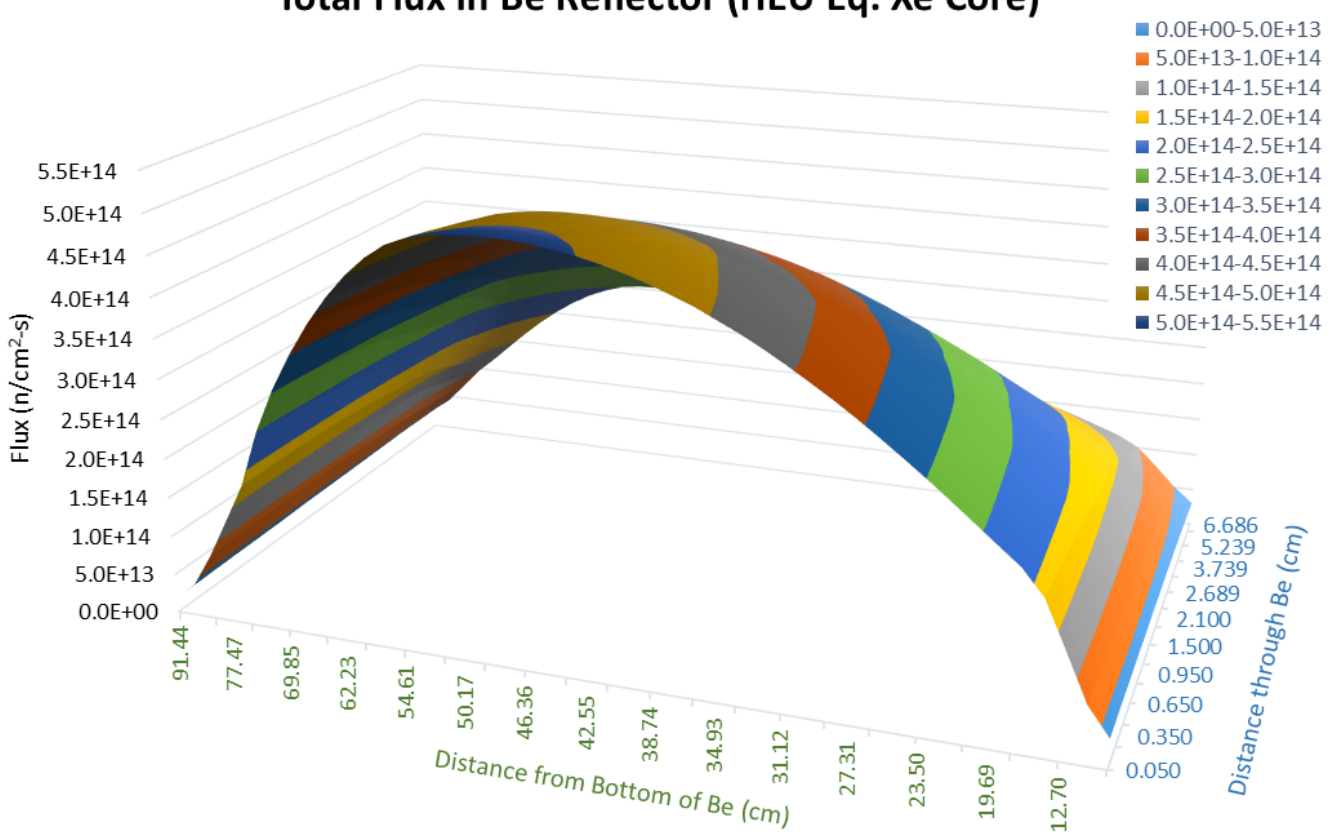

Figure A.5. Total flux in beryllium reflector (HEU core at equilibrium Xe)

Flux for E > 1 MeV in Be Reflector (HEU Eq. Xe Core) $\quad-0.0 E+00-1.0 E+13$

ㅁ. $1.0 \mathrm{E}+13-2.0 \mathrm{E}+13$

- $2.0 \mathrm{E}+13-3.0 \mathrm{E}+13$

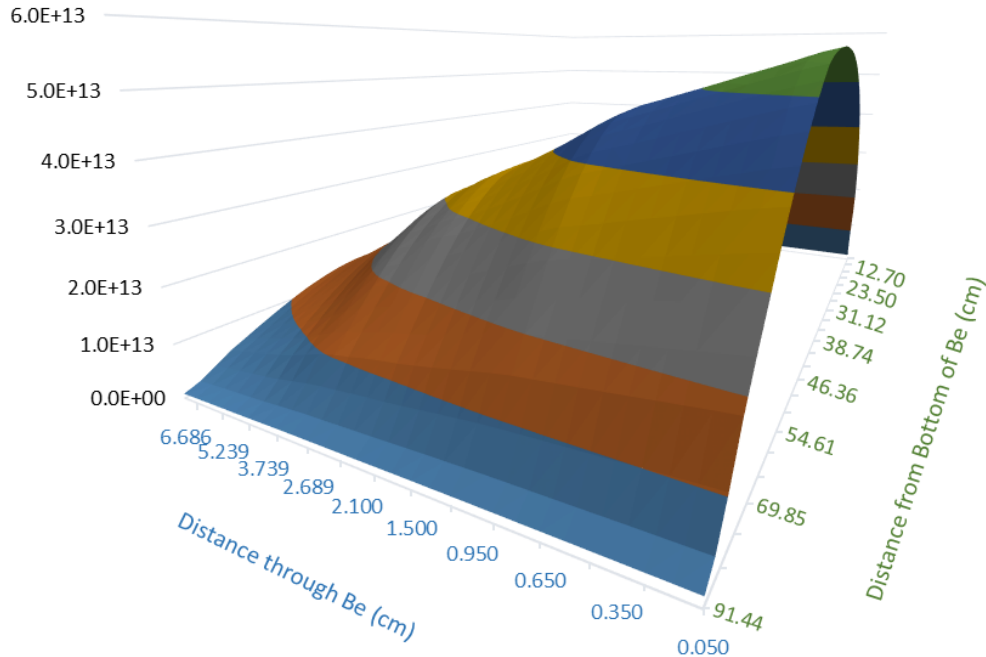

- $3.0 \mathrm{E}+13-4.0 \mathrm{E}+13$

- $4.0 \mathrm{E}+13-5.0 \mathrm{E}+13$

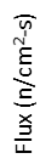

- 5.0 $\mathrm{E}+13-6.0 \mathrm{E}+13$

Figure A.6. Flux for $\mathrm{E}>1.0 \mathrm{MeV}$ in beryllium Reflector (HEU core at equilibrium Xe) 
Flux for $\mathrm{E}>\mathbf{1 0 0} \mathbf{k e V}$ in Be Reflector (HEU Eq. Xe Core) $=0.0 \mathrm{E}+00-2.0 \mathrm{E}+13$

- $2.0 \mathrm{E}+13-4.0 \mathrm{E}+13$

- $4.0 \mathrm{E}+13-6.0 \mathrm{E}+13$

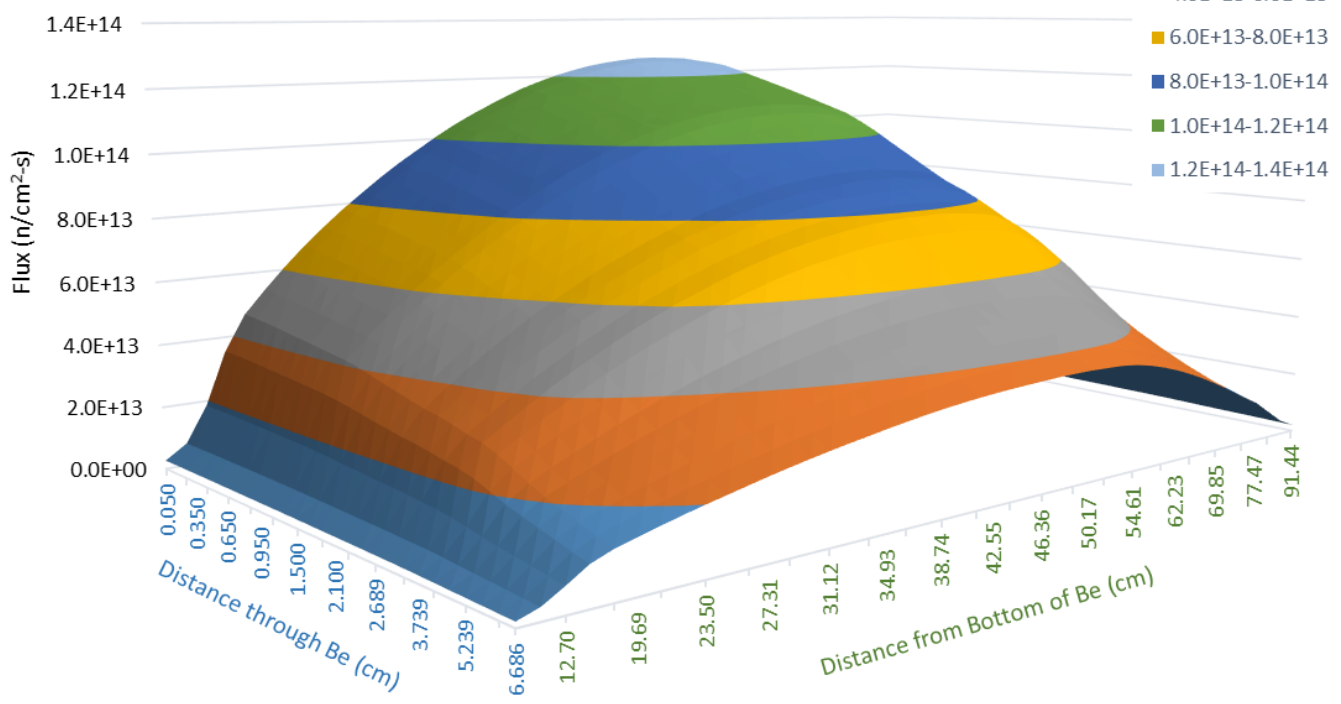

Figure A.7. Flux for $\mathrm{E}>0.1 \mathrm{MeV}$ in beryllium reflector (HEU core at equilibrium Xe)

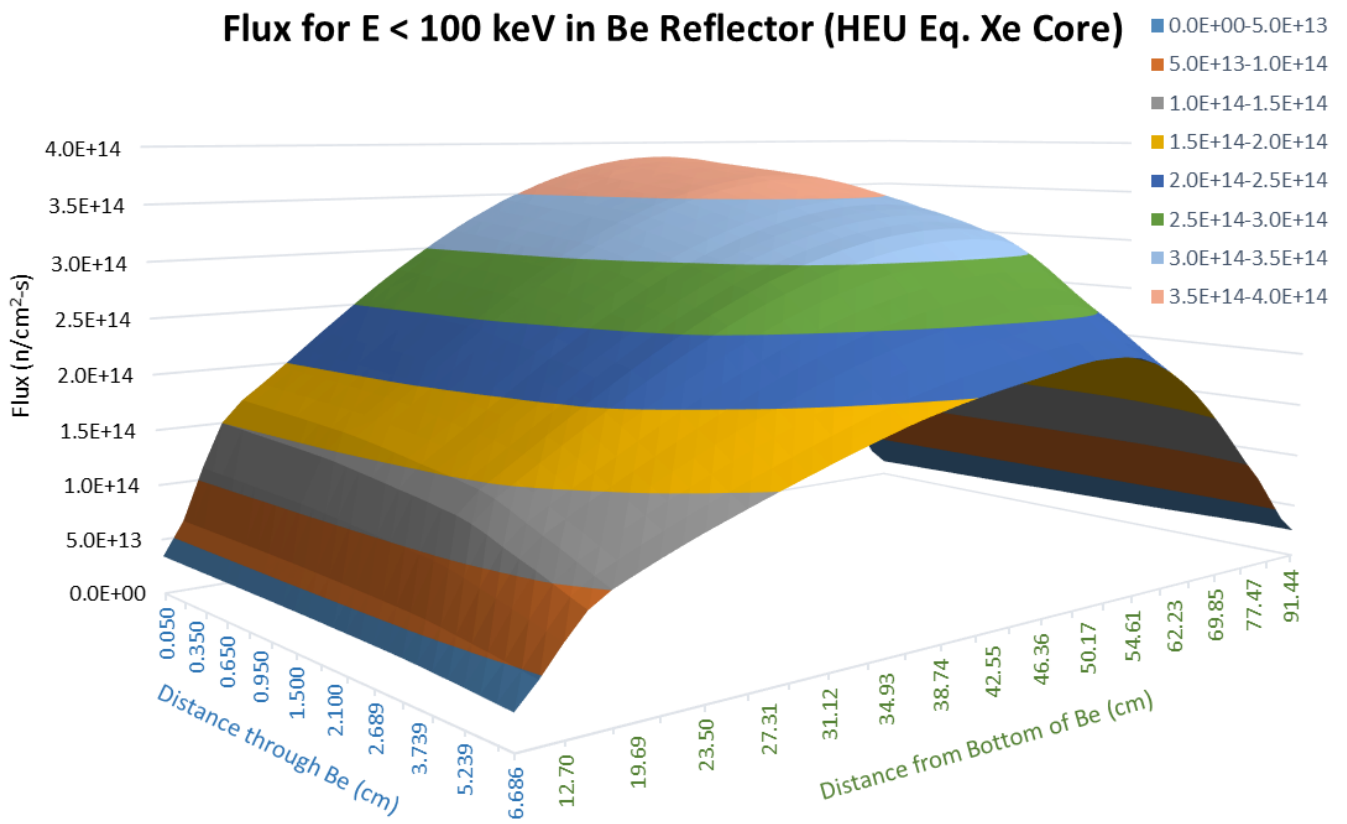

Figure A.8. Flux for $\mathrm{E}<0.1 \mathrm{MeV}$ in beryllium reflector (HEU core at equilibrium Xe)

The neutron spectra at two locations in the reflector were calculated to demonstrate how the energy distribution changes with position. Figure A.9 illustrates the locations selected for the neutron spectrum results and Figure A.10 provides the spectra at each location. It can be seen that the fast neutron flux decreases by up to $80 \%$ from the inner surface at the core midplane to the outermost surface at the bottom of the reflector. 


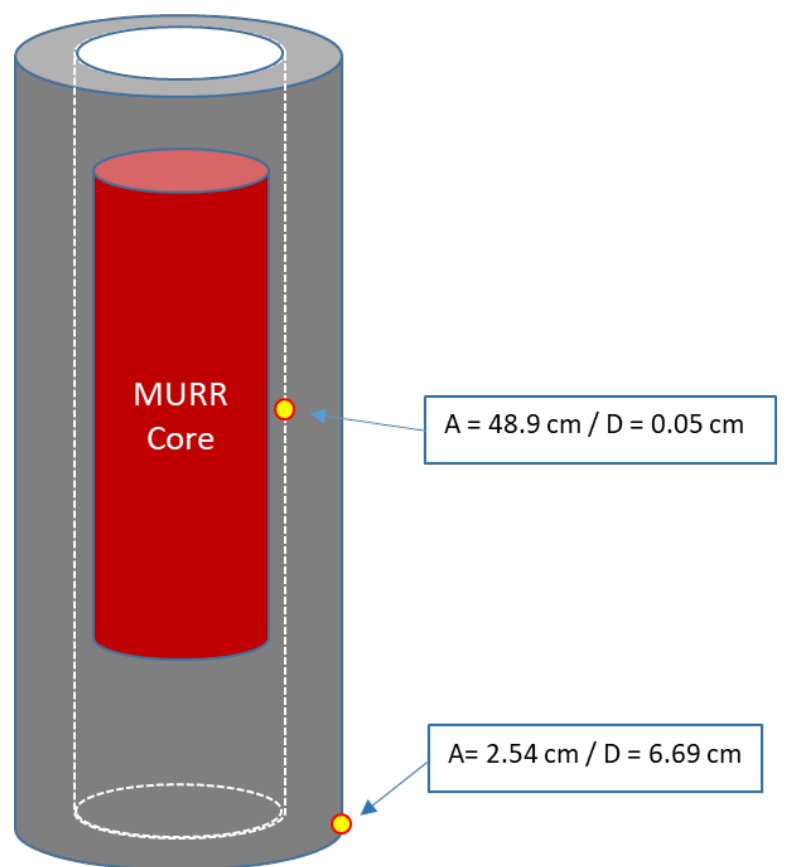

Figure A.9. Location of neutron flux spectra plots in beryllium reflector

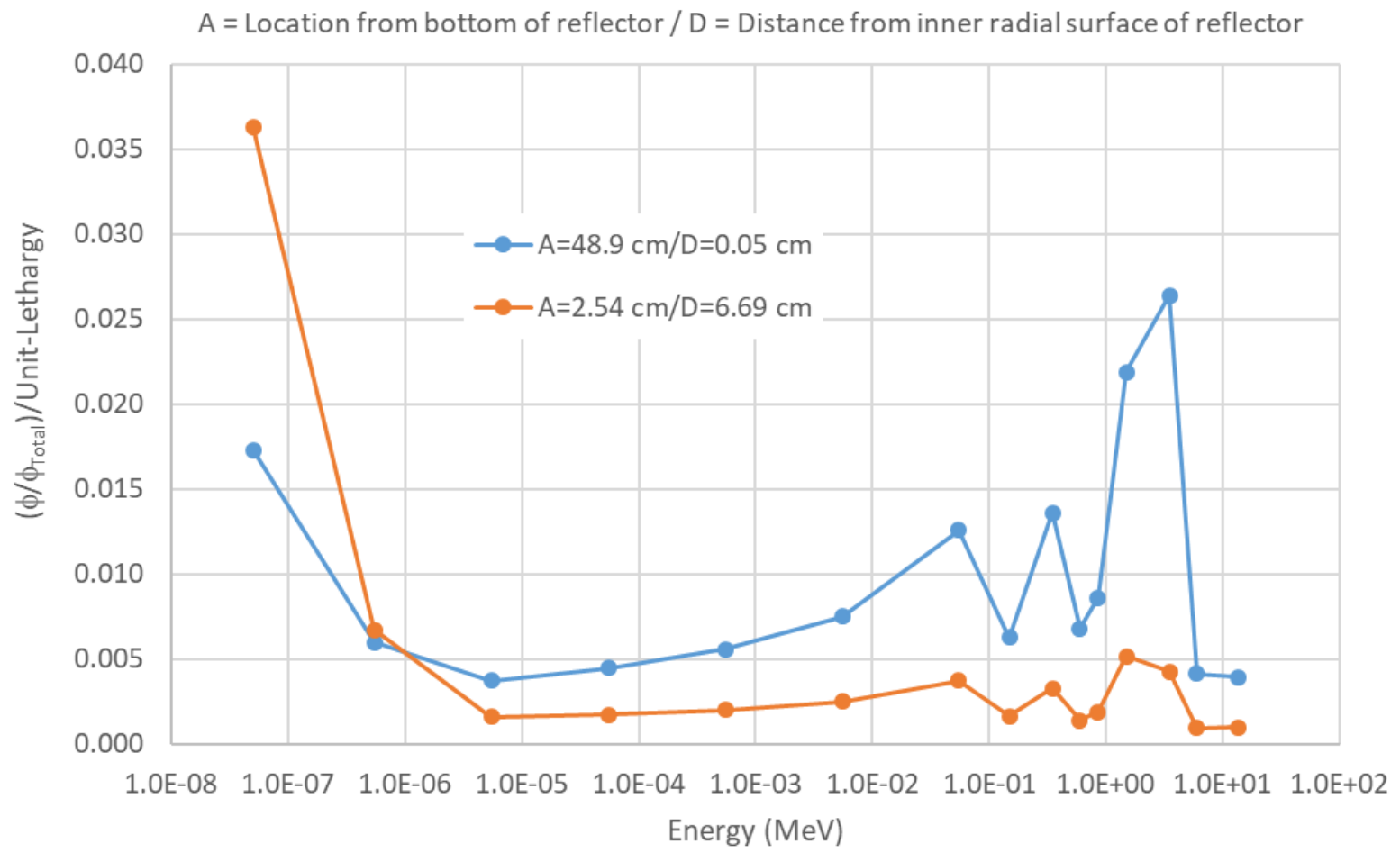

Figure A.10. Neutron flux by location in MURR reflector (HEU core at equilibrium Xe)

It is notable that regardless of position, the majority of the neutron flux in the reflector is from neutrons with $\mathrm{E}<100 \mathrm{keV}$ (generally $>75 \%$ of the total flux), although Figure A.11 shows that the Boral ${ }^{\circledR}$ control blades positioned between the core and reflector do cause a localized decrease in the epi-thermal neutron fraction. For the xenon-free core at BOC the control blades more deeply inserted, so the effect is even larger. However, while only about $20 \%$ of the neutrons in the reflector have 
energies $>100 \mathrm{keV}$, the high energy neutrons interact with the beryllium through threshold reactions to produce more than $90 \%$ of the helium which contributes to reflector swelling and about $85 \%$ of the damage to the reflector.
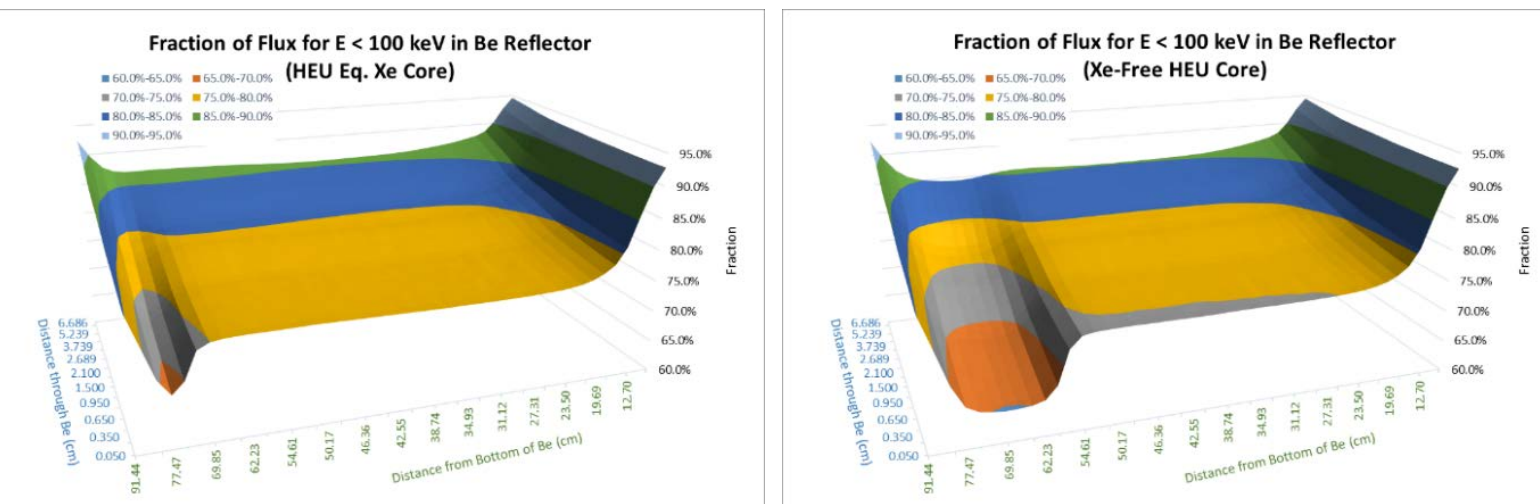

Figure A.11. Fraction of Flux for $\mathrm{E}<0.1 \mathrm{MeV}$ in beryllium reflector for $\mathrm{HEU}$ core at eq. Xe and Xe-free states

\section{A.4 Helium Production: Beryllium Reflector with HEU Core}

Helium-4 is produced primarily from three reactions: 1$)(n, 2 n)$ reactions in Be-9, 2) $(n, \alpha)$ reactions in Be-9, and 3) (n,T) reactions in Li- 6 as a by-product of the beryllium depletion via the $(n, \alpha)$ reaction. Analysis also shows that there is a small amount of He-4 produced from a photon-induced $(\gamma, 2 \alpha)$ reaction in the beryllium. The reaction rates at the axial position of the core mid-plane are shown in Figure A.12. It should be noted that the Be-9 $(n, 2 n)$ reaction produces two He-4 atoms per reaction, compared with a yield of 1 for the Be-9 $(n, \alpha)$ and Li-6 (n,T) reactions. Consequently, the Be-9 (n,2n) reaction dominates the total helium production in the reflector.

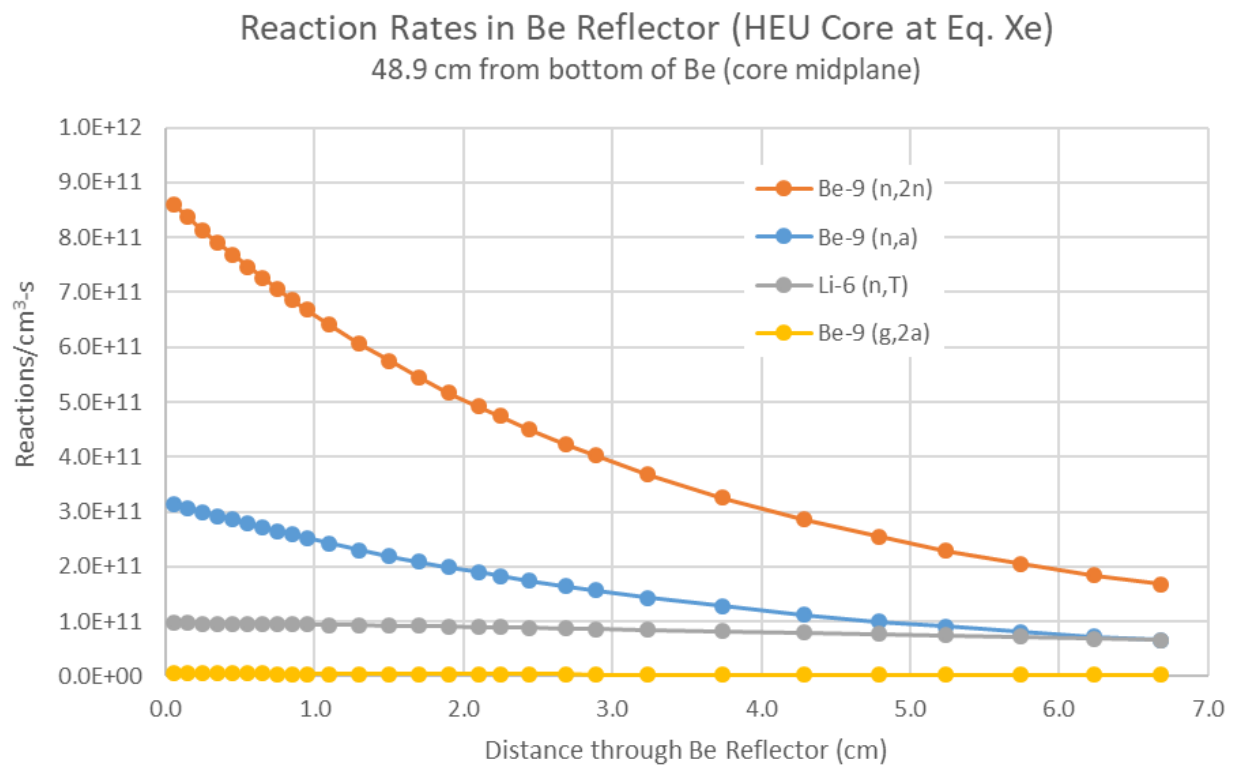

Figure A.12. Reaction rates in beryllium reflector (HEU core at equilibrium Xe)

Details of each of the reactions which produce He- 4 are described below. The reaction rates profiles are shown in Figure A.13. 


\section{Primary Reactions in Beryllium Reflector for He-4 Production}

Be-9 $(n, 2 n)$ Reaction Rate

The $(n, 2 n)$ reaction in Be-9 is a threshold reaction. The reaction rate peaks at the interface with the gap between the core and the reflector. It is dominated by neutrons with $\mathrm{E}>2 \mathrm{MeV}$, which accounts for $99.9 \%$ of the total reaction rate. All $(n, 2 n)$ reactions in Be- 9 occur for neutrons with $\mathrm{E}>1 \mathrm{MeV}$.

\section{Be-9 $(n, \alpha)$ Reaction Rate}

The $(n, \alpha)$ reaction in Be-9 is also a threshold reaction. The reaction rate peaks at the interface with the gap between the core and the reflector. It is dominated by neutrons with $\mathrm{E}>2 \mathrm{MeV}$, which accounts for more than $75 \%$ of the total reaction rate. All $(n, \alpha)$ reactions in Be-9 occur for neutrons with $\mathrm{E}>1 \mathrm{MeV}$.

\section{Li-6 $(n, T)$ Reaction Rate}

At equilibrium concentrations of $\mathrm{Li}-6$, the $(\mathrm{n}, \mathrm{T})$ reaction rate peaks at the interface with the gap between the core and the reflector. Unlike the threshold reactions in Be-9, almost all (n,T) reactions in Li- 6 occur at energies $<100 \mathrm{keV}$.

\section{Be-9 $(\gamma, 2 \alpha)$ Reaction Rate}

The photonuclear $(\gamma, 2 \alpha)$ reaction from high-energy photons in Be-9 produces a very small amount of He-4 in the reflector. The reaction rate is more than two orders of magnitude lower than the $(n, 2 n)$ reaction and about a factor of 50 lower than the $(n, \alpha)$ reaction rate in beryllium.

The total production of He-4 from the neutron and photon reactions in the beryllium was estimated assuming an irradiation period of 8 calendar years ( 7.2 full power years) and a constant rate of helium gas production from the reactions described above. This analysis is based on the assumption that 1 ) the source flux from the HEU core at equilibrium xenon conditions is constant over the irradiation of the reflector and 2) that the flux and spectrum in the reflector does not change with reflector age. These are both reasonable assumptions given that the typical operation cycle for MURR is relatively fixed.

Figure A.14 shows the distribution of the He-4 gas in the beryllium reflector with the HEU core produced by the reactions identified above. The equilibrium xenon core condition was selected since it corresponds to the burnup state and approximate control blade position which exist for most of the typical 6.3 day operating cycle. The analysis estimates a peak helium concentration after 8 years of operation at these conditions (7.2 full-power years) of 4,005 appm at the inner surface of the reflector at the core mid-plane. The majority of the helium production is from the Be-9 $(n, 2 n)$ reaction (> $80 \%)$. 

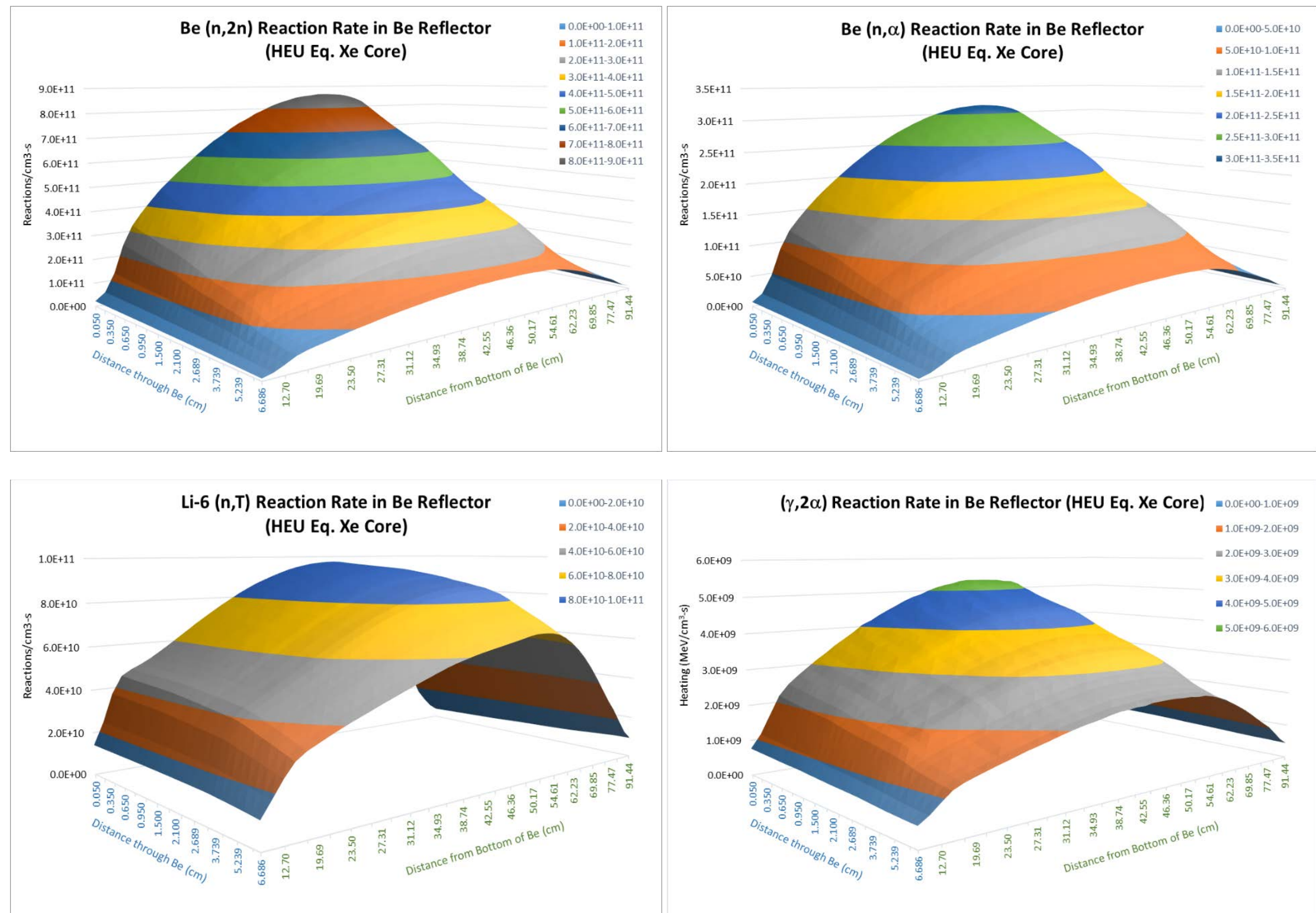

Figure A.13. Reaction rates in beryllium reflector (HEU core at equilibrium Xe) 


\section{Total He-4 Prodced in Be Reflector (8 CY)} (HEU Eq. Xe Conditions)

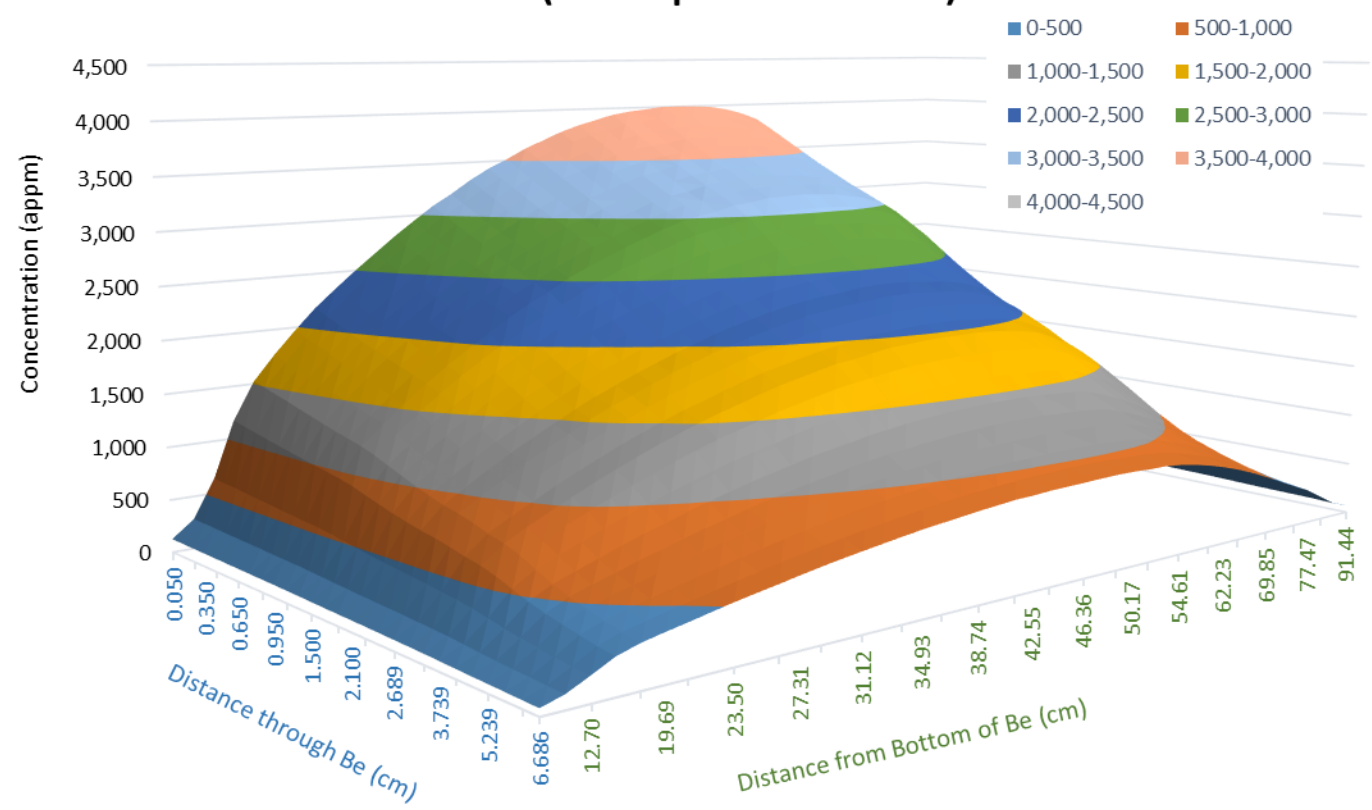

Figure A.14. Total He-4 produced in beryllium reflector at 8 years of operation with HEU core at equilibrium Xe

The average He-4 produced in the reflector after 8 years operation (7.2 full-power years) was calculated by homogenizing the detailed spatial results from the MCNP5 analysis over the entire reflector. Table A. 2 provides a summary of the sources of the He-4 produced in the reflector after 8 years operation with the HEU core predicted by the analysis with MCNP5 reaction rates. The results confirm that more than $90 \%$ of the He-4 is produced from neutron and photon reactions with Be- 9 . A small fraction $(<9 \%)$ of the He-4 production is from $(n, T)$ reactions with the Li- 6 by-product of the $(n, \alpha)$ reaction with Be-9.

Table A.2. He-4 gas concentration predicted by MCNP5 analysis in reflector with HEU core at 8 years operation

\begin{tabular}{|l|c|c|}
\hline Source Reaction & Concentration (appm) & Fraction \\
\hline Be-9 $(n, 2 n)$ & 769 & $75.9 \%$ \\
\hline Be-9 $(n, \alpha)$ & 148 & $14.6 \%$ \\
\hline Li-6 $(n, T)$ & 89 & $8.8 \%$ \\
\hline Be-9 $(\gamma, 2 \alpha)$ & 7 & $0.7 \%$ \\
\hline Total & 1013 & $100 \%$ \\
\hline
\end{tabular}

Predicted concentrations of He-4+T (total gas) in the reflector with the HEU core were reported in [6]. The data were provided in units of ppm (parts per million by mass). Adjusting to units of appm (parts per million by atom), the average gas concentration predicted by [6] is $1178 \mathrm{appm}$. Since the Li-6 $(\mathrm{n}, \mathrm{T})$ reaction produced both one $\mathrm{T}$ and one He-4 atom, the $\mathrm{T}$ fraction in the reflector can be estimated from the MCNP5 results presented in Table A.2 as $8 \%(=89 /(89+1013))$ of the total gas concentration. Using this value for the $\mathrm{T}$ fraction to adjust the results predicted by [6], the He- 4 in the 
reflector is 1084 appm, which agrees to within $7 \%$ of the He-4 concentration predicted from the MCNP5 reaction rate analysis. Users should be particularly careful to note units of ppm or appm when comparing predictions of gas concentrations in beryllium reflector materials to avoid mistakes in calculations of the reflector swelling with irradiation.

MURR operates for a brief period each week (weekly operating cycle of 6.3 full-power days) at xenonfree conditions. At these conditions at reactor startup, the control blades are positioned about 6 inches lower than at equilibrium xenon conditions. Under these xenon-free conditions, the neutron flux profile is more axially-peaked, and peaked at a lower axial position, as shown in Figure A.15 for the HEU core. The change in the axial flux distribution results in higher local production rates of helium, especially near the inner surface of the reflector, as shown in Figure A.16, which plots the most relevant reaction rates by radial position and the axial position of the peak flux.

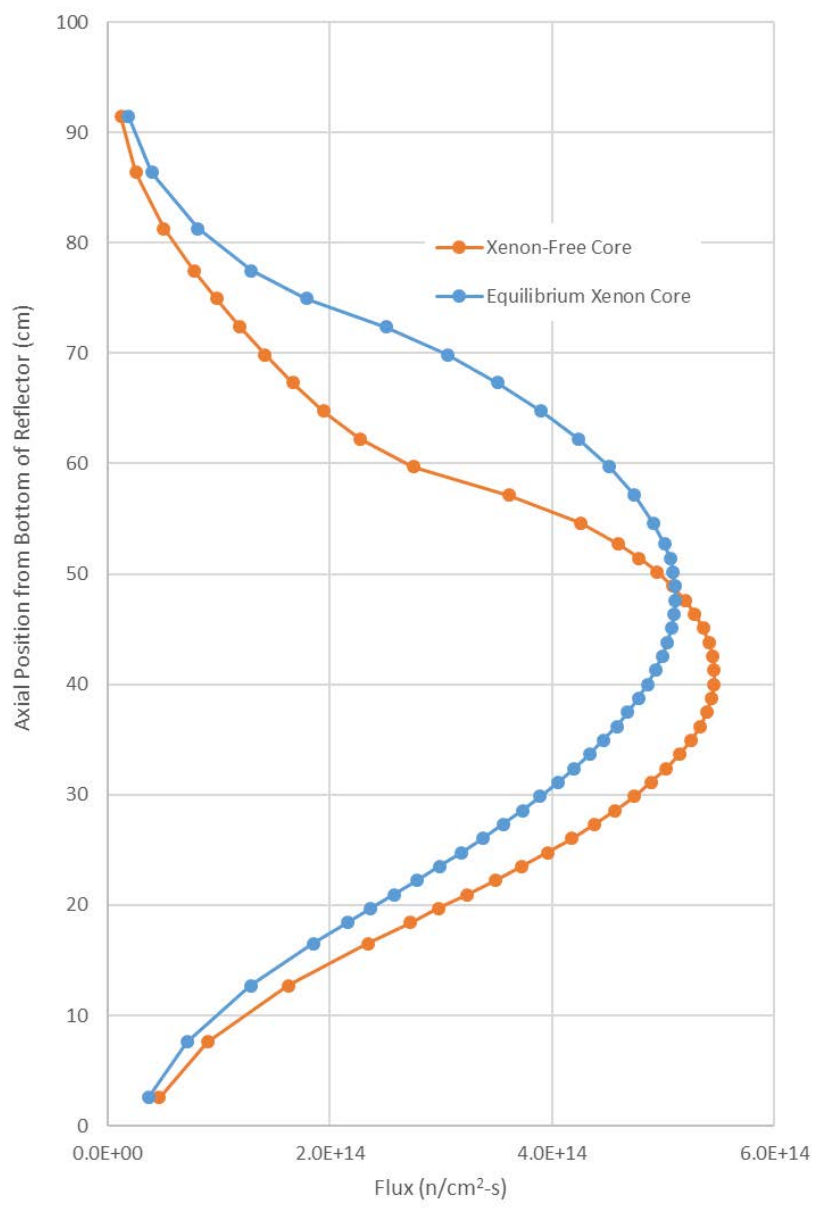

Figure A.15. Total flux at inner surface of MURR beryllium reflector 


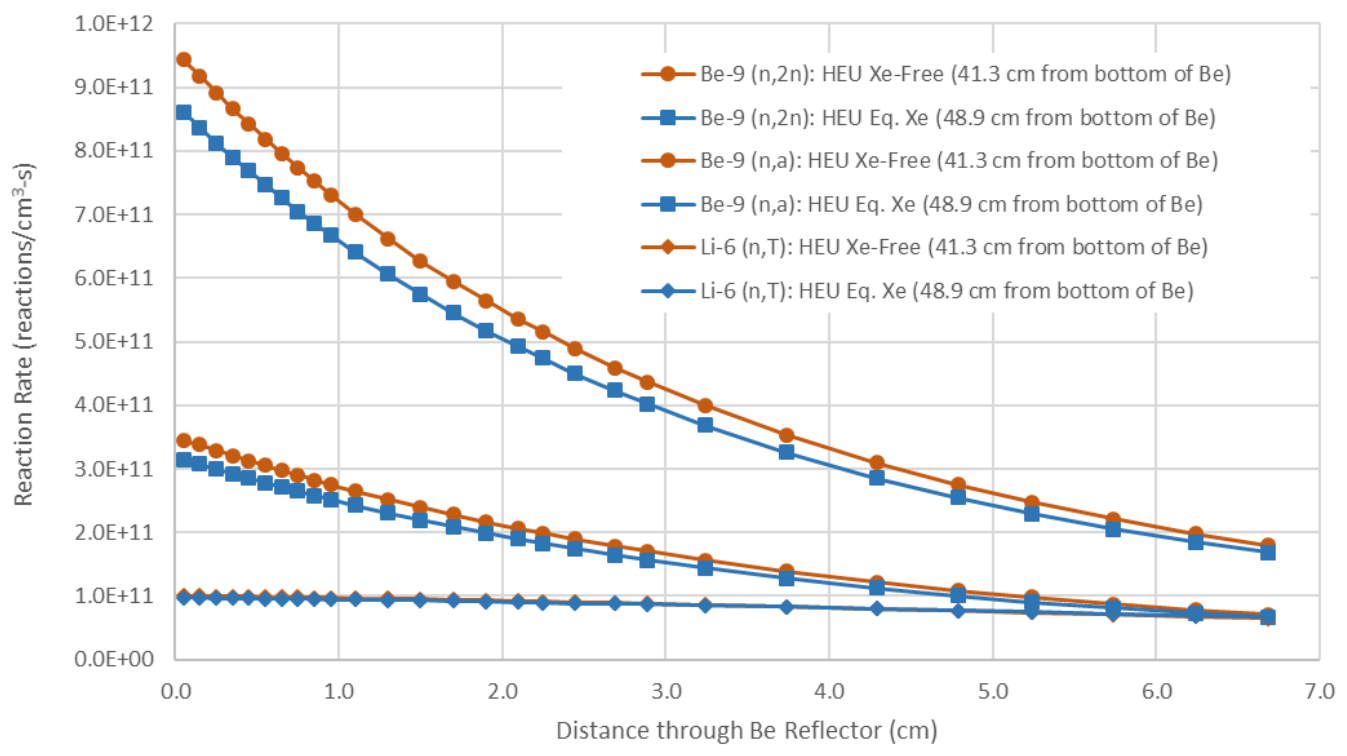

Figure A.16. Reaction rates in beryllium reflector with HEU core

For an estimate of the total He-4 concentration in the beryllium reflector that takes into account the change in the core conditions during typical operations, a linear combination of the helium production rates at xenon-free and equilibrium xenon conditions for 2.0 and 4.3 full-power days each, respectively, was used. The maximum He-4 concentration in the reflector with the HEU core after 8 years operation using this method of combination is 4,076 appm, which is $1.8 \%$ larger than the helium concentration predicted based on the equilibrium xenon conditions only. It should be noted, however, that this approach is overly conservative since it assumes that the more deeply inserted control blade position at xenon-free conditions persists for 2 full-power days, when in reality the control blades will be withdrawn quickly during this period of the cycle to compensate for the reactivity loss from increasing xenon concentration in the core. After equilibrium xenon conditions are reached, the control blades continue to slowly move out of the active core region to compensate for fuel depletion and the axial flux profile throughout the remainder of the cycle becomes increasingly less axially-peaked. Consequently, the He-4 concentration, as well as irradiation damage and beryllium heating, is considered to be adequately conservative when calculated assuming equilibrium xenon conditions throughout the lifetime of the reflector.

Lastly, as a comparative check of the MCNP5 analysis relative to measurements reported by others in literature, the He-4 concentration predicted by the current analysis with MCNP5 was compared with that reported for beryllium irradiation in literature. Figure A.17 shows the measured and calculated He- 4 concentration as a function of fluence for neutrons with $\mathrm{E}>0.1 \mathrm{MeV}$. Note that the data reported in Figure A.17 includes helium produced by neutrons of all energies; the data are only plotted against fast fluence as a way of presenting the data. The many data points from the MCNP5 analysis with the HEU core correspond to the nearly 1,300 (45x28) individual tally zones. At fast neutron fluence levels relevant for the MURR reflector (up to $3^{*} 10^{22} \mathrm{n} / \mathrm{cm}^{2}$ ), the He-4 concentrations predicted by the MCNP5 analysis are somewhat lower than the reported measurements (up to $50 \%$ less), but are considered in reasonable agreement with the measured data given uncertainties and the likely differences in neutron spectrum between MURR and the test conditions for the HFIR, HFBR, and SM reactor measurements. 


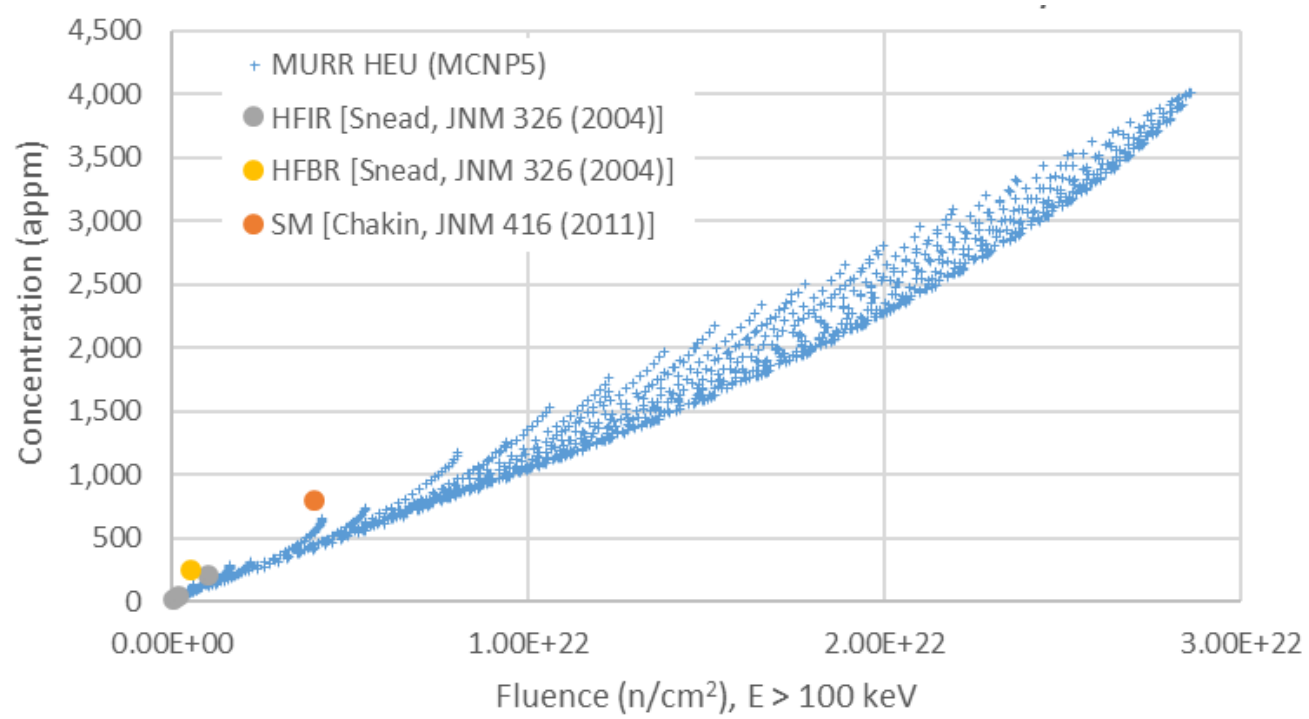

Figure A.17. He-4 concentration in irradiated beryllium

\section{A.5 Neutron Irradiation Damage: Beryllium Reflector with HEU Core}

Irradiation damage was estimated in the MCNP5 analysis using a flux multiplier tally with the $\mathrm{MT}=444$ reaction in Be-9 (ENDF/B-VII cross section library 4009.70c). As can be seen in Figure A.18, the damage rate in displacements $/ \mathrm{cm}^{3}$-s follows a behavior similar to the total neutron flux (see Figure A.2) and is greatest at the inner surface of the reflector near the core mid-plane. Figure A.19 shows that the majority of the damage from atomic displacement occurs for neutron energies greater than $100 \mathrm{keV}$, although about 20\% of the reflector damage does occur from neutrons with energies less than $100 \mathrm{keV}$. It is thus observed that beryllium damage is caused by both epi-thermal as well as fast neutrons.

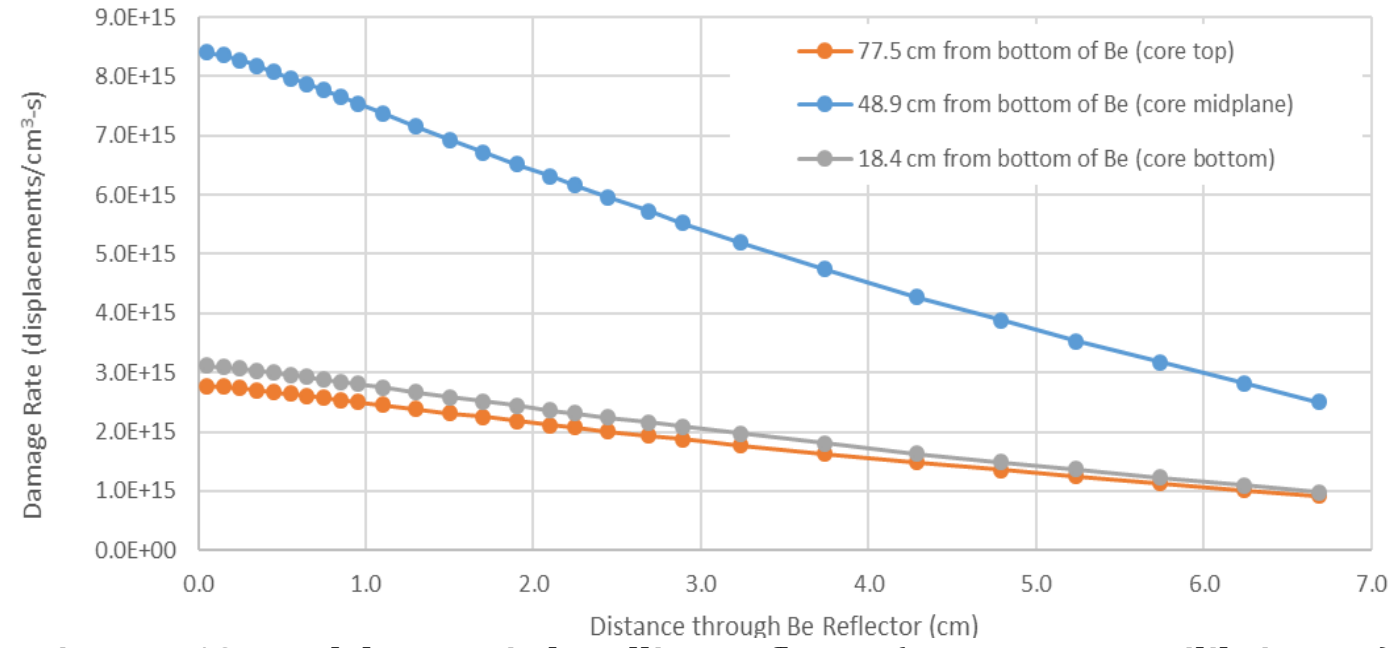

Figure A.18. Total dpa rate in beryllium reflector (HEU core at equilibrium Xe) 

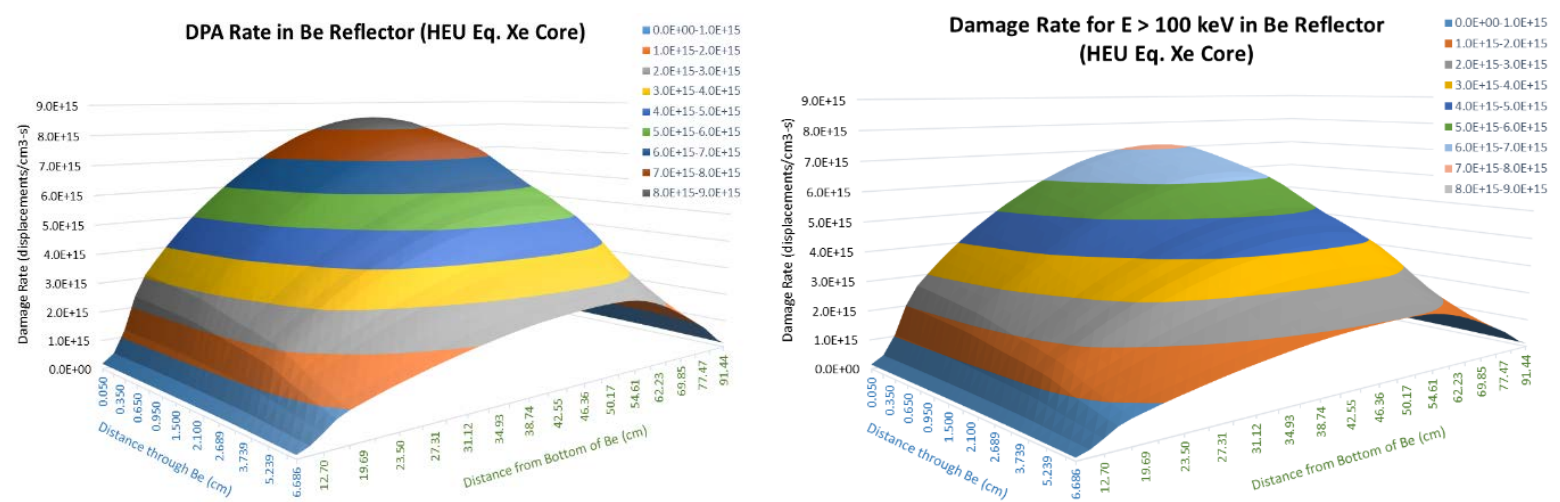

Figure A.19. Damage rate in beryllium reflector (HEU core at equilibrium Xe)

The damage rate was subsequently used to predict the total irradiation damage in the reflector after 8 years of operation, assuming that the damage rate is constant and is not affected by changes in the neutron spectrum or magnitude over the life of the reflector. The analysis also assumes that the neutron source from the core is constant, and corresponds to the equilibrium xenon HEU core. The results shown in Figure A.20 for the HEU core show that the damage in displacements per atom (dpa) peaks at the inner surface of the reflector near the core mid-plane. The maximum damage in the reflector with HEU at 8 years of operation is $15.7 \mathrm{dpa}$.

As for the He-4 concentration in the irradiated beryllium, a comparative check of the MCNP5 predictions of beryllium damage can be compared relative to measurements reported by others in literature. Figure A.21 compares the beryllium damage versus fast neutron fluence for measured data and predicted for the MURR reflector with HEU. The comparison shows very good agreement between the predicted and measured beryllium damage at fluence levels relevant to the MURR reflector.

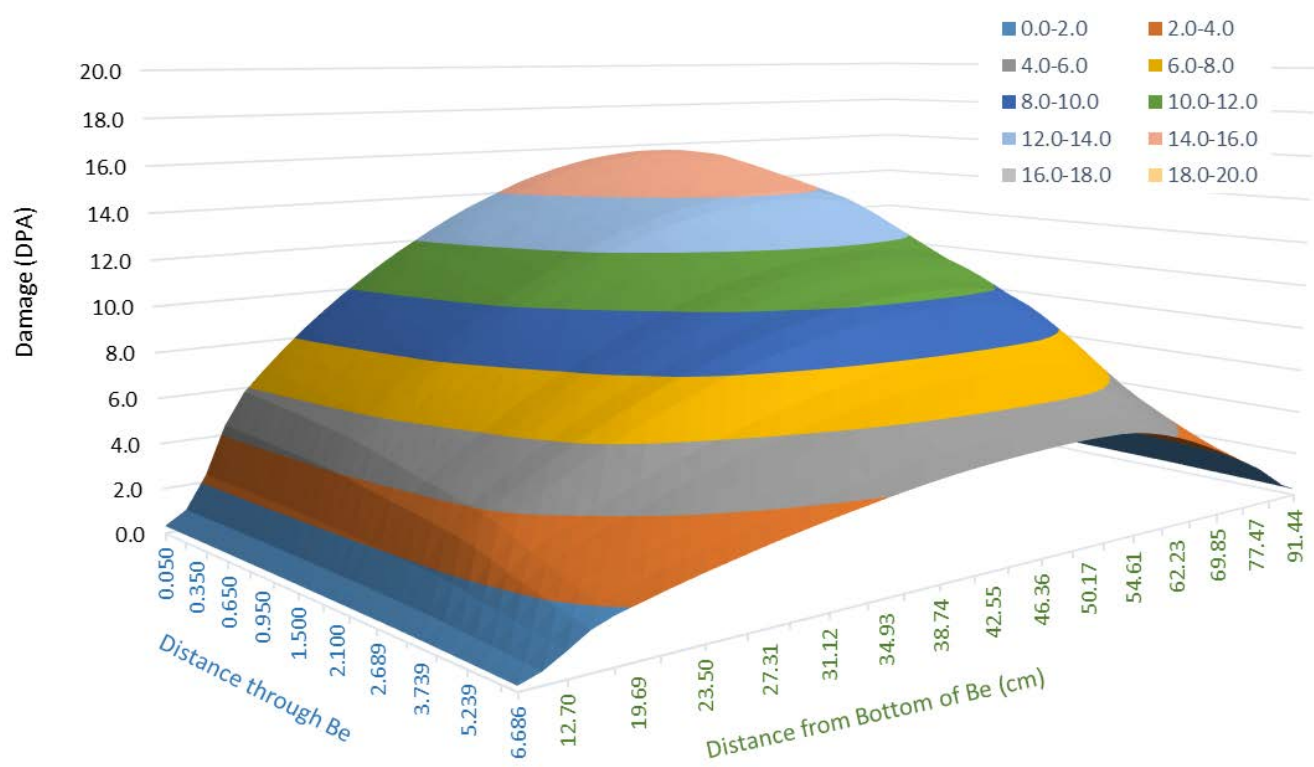

Figure A.20. Damage in beryllium reflector at 8 years of operation (HEU core at equilibrium Xe) 


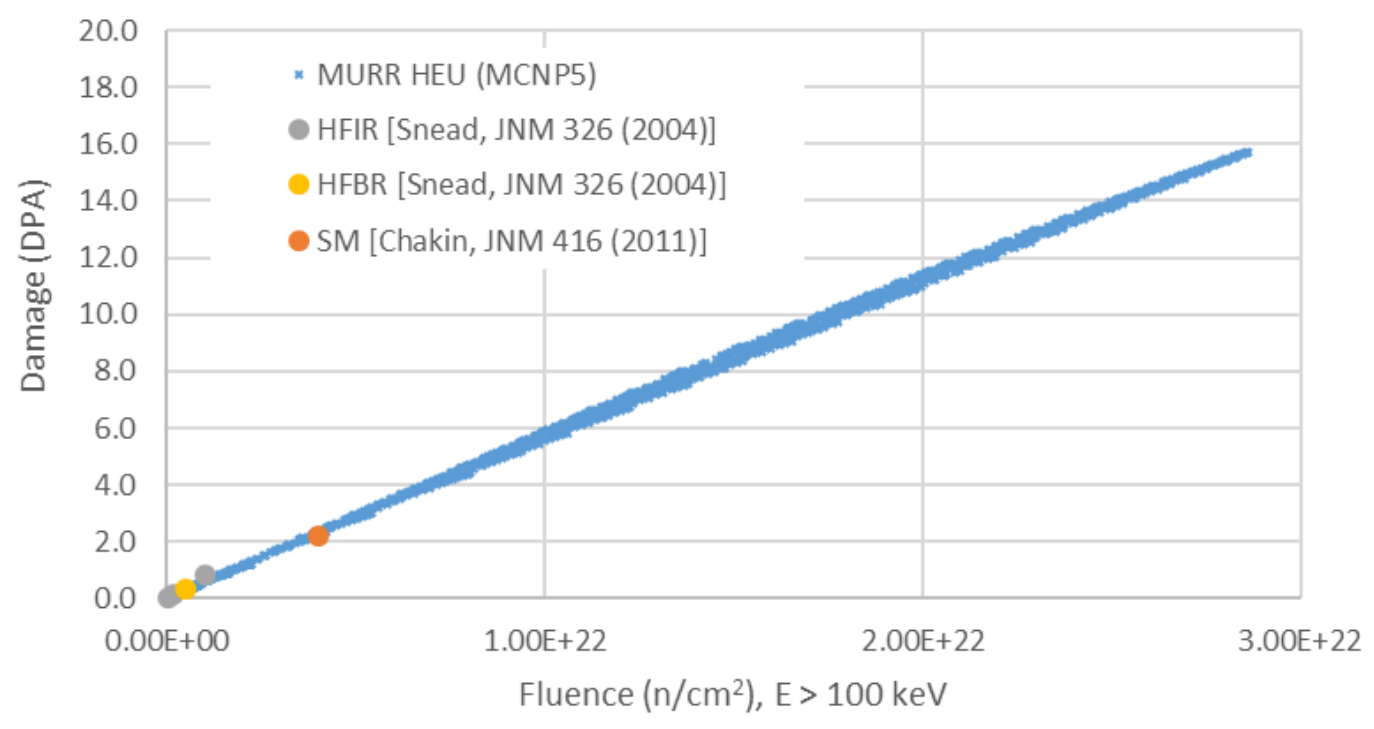

Figure A.21. Neutron damage in irradiated beryllium

\section{A.6 Neutron and Photon Heating: Beryllium Reflector with HEU Core}

During operation, the reflector will be heated from energy deposition from neutron and photon reactions. Heating tallies with neutron and photon transport were implemented in the MCNP5 model to predict the heating magnitude and distribution in the reflector.

Table A.3 provides a summary of the energy release values for U-235 fission from the ENDF/B-VI data. The total recoverable energy from fission events is $193.7 \mathrm{MeV} /$ fission. Combined with a typical energy deposition from excess neutron capture of 6 to $8 \mathrm{MeV}$, the total recoverable energy is $\sim 200 \mathrm{MeV} /$ fission. However, MCNP5 only includes the energy release from fission fragments (deposited locally in the fuel), prompt photons, and neutron scattering and capture reactions from excess neutrons in heating tallies for MCNP5 calculations with neutron and photon transport. Delayed photons and $\beta$ 's released from fission reactions, which together amount to $12.83 \mathrm{MeV} /$ fission, are neglected.

Table A.3. Energy Release from U-235 fission (MeV/fission)

\begin{tabular}{|l|r|}
\hline Fission Products & 169.10 \\
\hline Neutrons & 4.79 \\
\hline Prompt photons & 6.97 \\
\hline Beta & 6.50 \\
\hline Delayed photons & 6.33 \\
\hline Neutrinos (not recoverable) & 8.75 \\
\hline Total recoverable energy from fission & 193.69 \\
\hline
\end{tabular}

To include the energy deposition from these components of the total recoverable fission energy, simple assumptions can be made to estimate their contribution. It can be assumed that the $\beta$ particles will not travel outside of the fuel, so that any fission energy release carried by these particles will deposit locally in the fuel and can be neglected from reflector heating estimates. The delayed photons, 
on the other hand, will transport throughout the system and it is reasonable to assume they will deposit their $6.33 \mathrm{MeV} /$ fission with the same distribution as the prompt photons.

Table A.4 provides a summary of the results of two MCNP5 calculations, one with neutron transport and a separate calculation with photon transport, along with the estimated contribution of the delayed photons to the reflector heating. Based on the two transport calculations, the average neutron heating in the reflector calculated after tally normalization to $10 \mathrm{MW}$ core power is $0.39 \mathrm{~W} / \mathrm{cm}^{3}$, while the prompt photon heating is $0.88 \mathrm{~W} / \mathrm{cm}^{3}$. The prompt photon heating can be used to predict the delayed photon heating using the ratio of the delayed and prompt photon energy release values, which is calculated to be $0.80 \mathrm{~W} / \mathrm{cm}^{3}$ in the reflector. From this, the heating in the reflector can be calculated as

$$
H_{i}^{T}=0.39 \times F_{i}^{n}+(0.88+0.80) F_{i}^{\gamma_{p}}
$$

where $H_{i}^{T}$ is the total heating in tally segment $I, F_{i}^{n}$ is the fraction of the neutron heating in tally segment $I$, and $F_{i}^{\gamma_{p}}$ is the fraction of the prompt photon heating in tally segment $i$.

Table A.4. Average heating in beryllium reflector with HEU core $\left(\mathrm{W} / \mathrm{cm}^{3}\right)$

\begin{tabular}{|l|c|}
\hline $\begin{array}{l}\text { Neutron Heating } \\
\text { (Neutron transport calculation) }\end{array}$ & 0.39 \\
\hline $\begin{array}{l}\text { Prompt Photon Heating } \\
\text { (Photon transport calculation) }\end{array}$ & 0.88 \\
\hline $\begin{array}{l}\text { Est. Delayed Photon Heating } \\
(=0.88 \times 6.33 \div 6.97)\end{array}$ & 0.80 \\
\hline
\end{tabular}

The total neutron and photon heating in the beryllium reflector with the HEU core is shown in Figure A.22. The peak energy deposition in the reflector is predicted to be $5.92 \mathrm{~W} / \mathrm{cm}^{3}$. The total reflector heating for the reactor at $10 \mathrm{MW}$ operating power is $173 \mathrm{~kW}$, or $1.7 \%$ of the reactor power.

Reflector heating with the HEU core at $10 \mathrm{MW}$ calculated with MCNP5 in the current work and in two independent calculations reported in [6], [99] are summarized in Table A.5. Each of these calculations are reasonably close to one another given that not all aspects of the modeling conditions are known. In the work by [6] the maximum reflector heating was reported as $4.65 \mathrm{~W} / \mathrm{cm}^{3}$, based on a 15-region discretization (3 radial regions $\times 5$ axial regions) of the reflector. For the same discretization, the maximum heating in the reflector calculated from the MCNP5 analysis performed here is about 4\% larger, or $4.79 \mathrm{~W} / \mathrm{cm}^{3}$. The average power density based on the analysis reported by Saddler is $1.92 \mathrm{~W} / \mathrm{cm}^{3}$, yielding a total reflector heating of $163 \mathrm{~kW}$, which is within $6 \%$ of the total reflector heating calculated here. In [99], Charak reported an estimated total reflector heating at $10 \mathrm{MW}$ reactor operation of $218 \mathrm{~kW}$ based on analyses that pre-date MURR operations with HEU. 


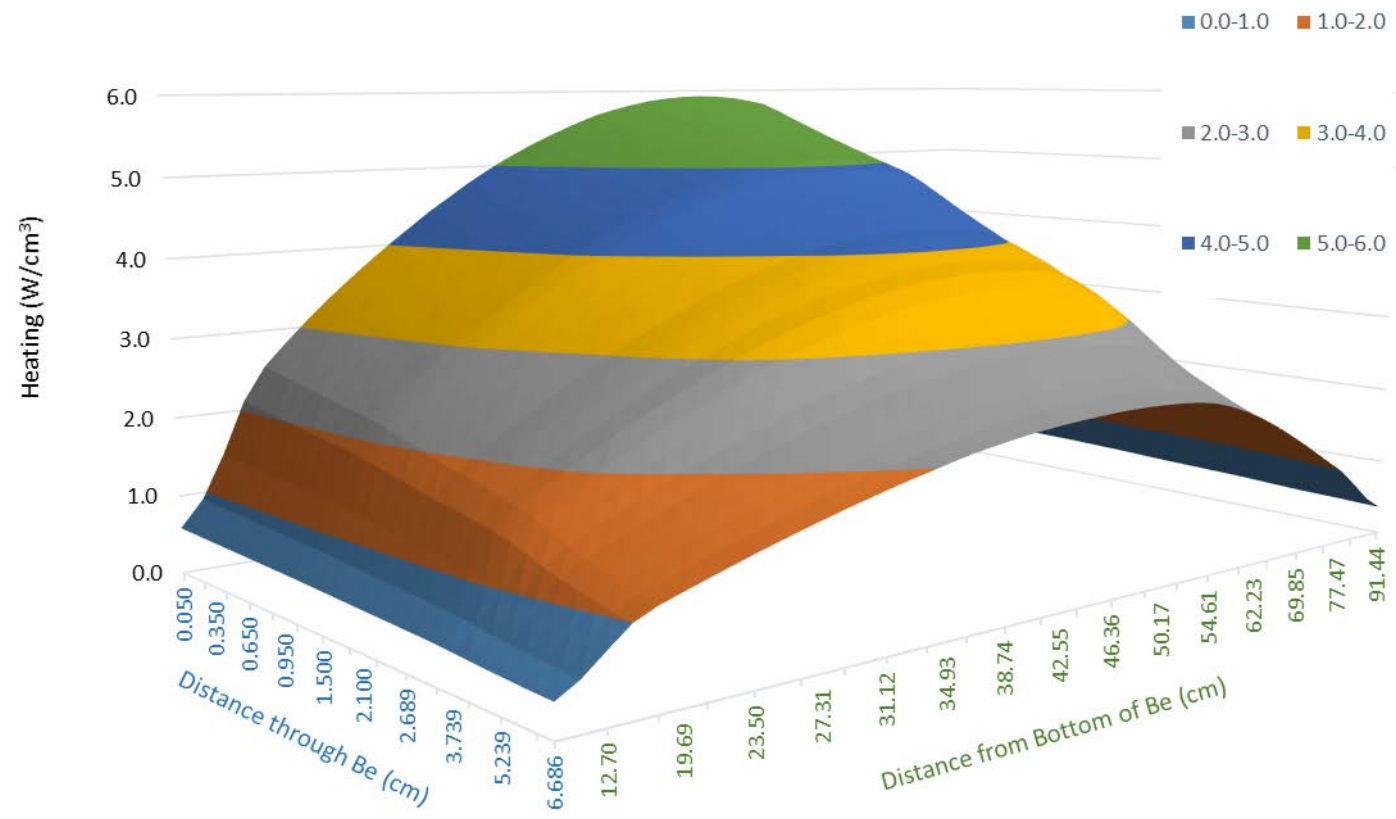

Figure A.22. Total heating in beryllium reflector (HEU core at equilibrium Xe)

Table A.5. Heating in MURR beryllium reflector with HEU core

\begin{tabular}{|l|c|c|c|}
\hline & $\begin{array}{c}\text { MCNP5 } \\
\text { (current work) }\end{array}$ & Reference [6] & Reference [99] \\
\hline $\begin{array}{l}\text { Maximum Heating, W/cm }{ }^{3}(28 \times 45 \\
\text { discretization) }\end{array}$ & 5.91 & & \\
\hline $\begin{array}{l}\text { Maximum Heating, W/cm } \\
\text { discretization) }\end{array}$ & 4.79 & 4.65 & \\
\hline Average Heating, W/cm & 2.07 & 1.92 & \\
\hline Total Heating, $\mathrm{kW}$ & 173 & 163 & 218 \\
\hline
\end{tabular}

\section{A.7 Neutron Flux: Beryllium Reflector with LEU Core}

As part of conversion to LEU, MURR will increase its operating power from $10 \mathrm{MW}$ to $12 \mathrm{MW}$. This power uprate is necessary to mitigate a loss of experimental performance following conversion. Without the power uprate, the increased mass of U-238 in the core decreases the neutron leakage to the experimental irradiation positions in the center flux trap and graphite reflector, which is located outside the beryllium reflector. Figure A.23 shows that though the power uprate is $20 \%$, the total neutron flux at the inner surface of the beryllium is increased by just $3 \%$ because of the increased parasitic capture in the U-238 in the core. Figure A.24 shows that the fast flux $(\mathrm{E}>1.0 \mathrm{MeV})$ increases by $9 \%$ due to the conversion and power uprate. The characteristics observed for the neutron flux in the reflector with the HEU core (decreasing flux and softening of the neutron spectrum with penetration into the reflector) are the same for the LEU core. 


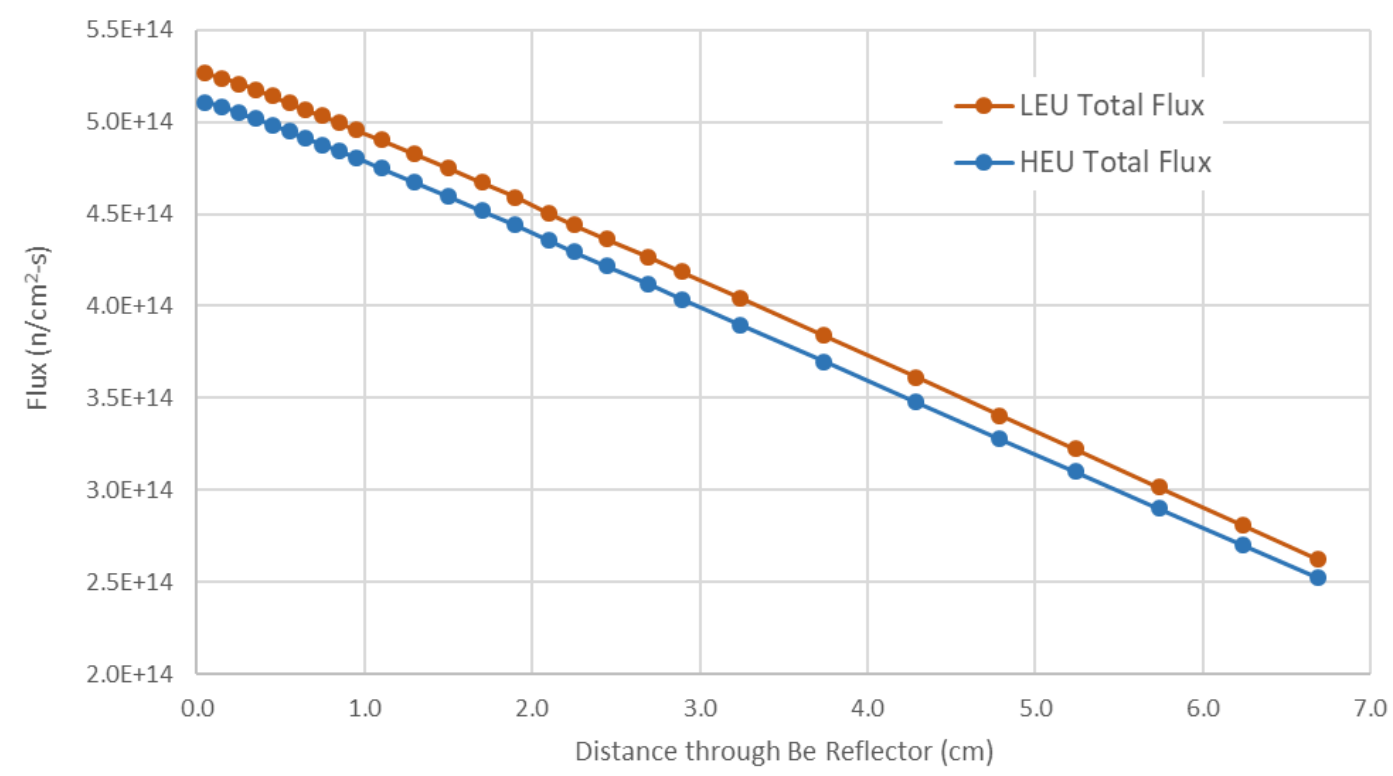

Figure A.23. Total flux in beryllium reflector at core mid-plane (equilibrium Xe conditions)

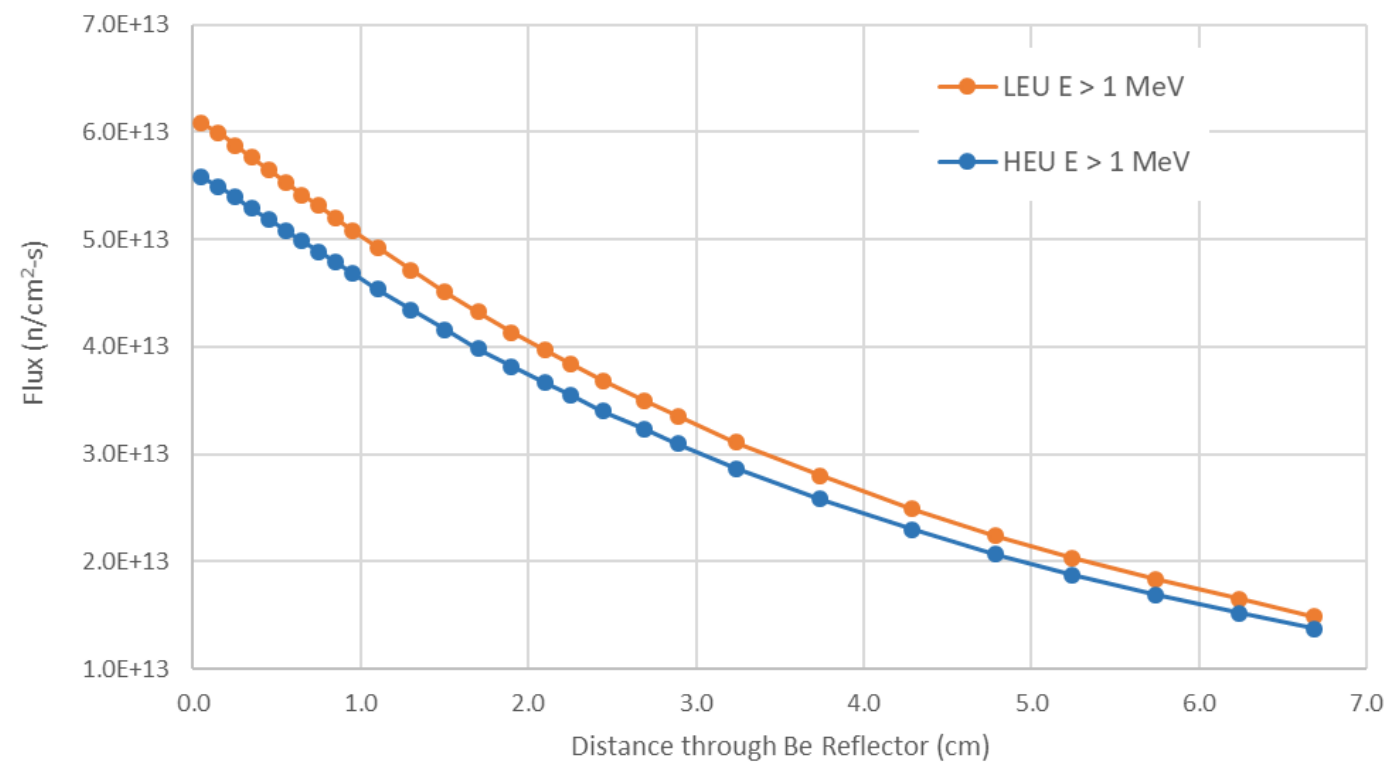

Figure A.24. Flux E $>1.0 \mathrm{MeV}$ in beryllium reflector at core mid-plane (equilibrium Xe conditions)

\section{A.8 Helium Production: Beryllium Reflector with LEU Core}

The reaction rates which lead to He-4 production with radial penetration in the reflector with the LEU core are displayed in Figure A.25. The trends are similar to those for the HEU core:

- the maximum production rate is at the inner surface of reflector near the core mid-plane, and

- the majority of helium production $(>75 \%)$ is from the $(n, 2 n)$ reaction in Be-9. 


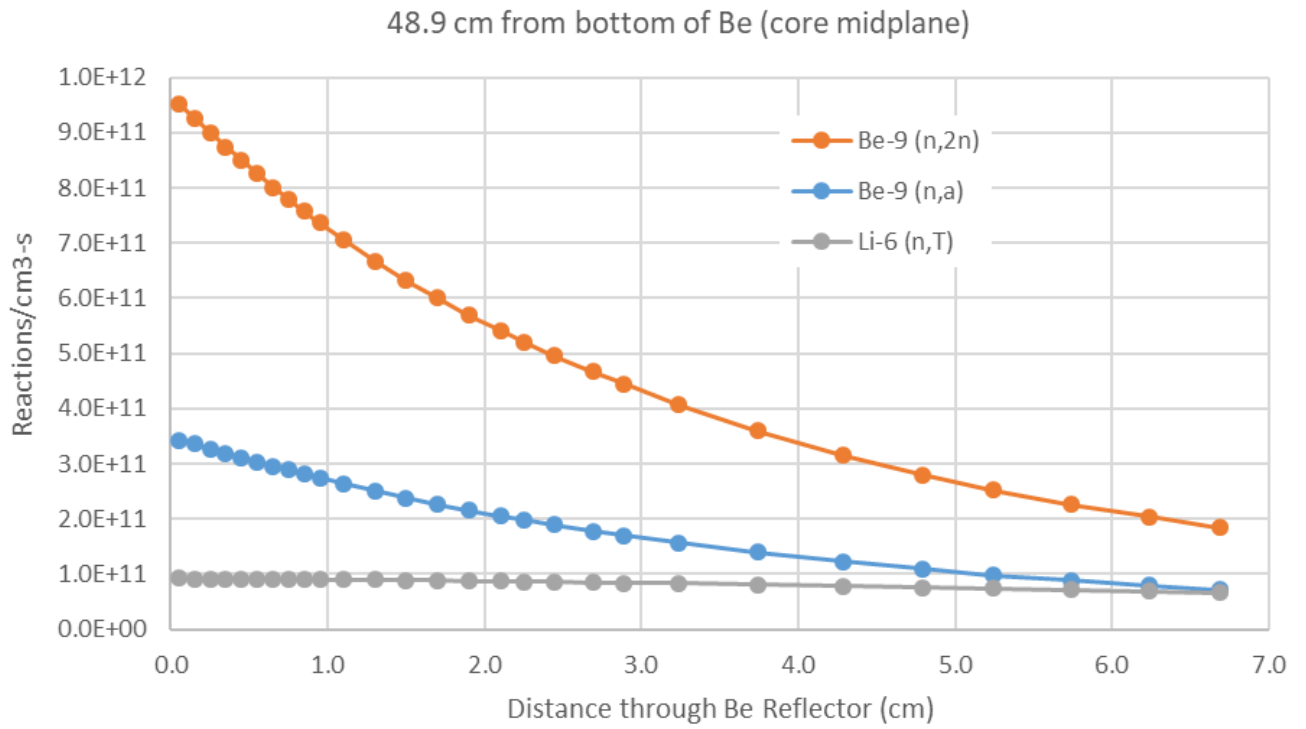

Figure A.25. Reaction rates in beryllium reflector (LEU core at equilibrium Xe)

As shown in Table A.6, the $(n, 2 n)$ and $(n, \alpha)$ reaction rates in Be-9 are $\sim 10 \%$ greater for the LEU core at $12 \mathrm{MW}$ compared to the $\mathrm{HEU}$ core at $10 \mathrm{MW}$. This is due to the increased fast flux $(\mathrm{E}>1.0 \mathrm{MeV})$ in the reflector following conversion. The $(\mathrm{n}, \mathrm{T})$ reaction rate in Li-6, however, is $\sim 5 \%$ less for the LEU core because of a decrease in the thermal flux in the reflector following conversion. The $(\gamma, 2 \alpha)$ reaction rate in the beryllium is $10.2 \%$ less with the LEU core compared to the HEU core; the photon flux in the reflector is lower following conversion due to greater attenuation of photons due to the larger uranium loading in the LEU core.

Table A.6. Reaction rates and neutron flux at inner surface of reflector at core mid-plane

\begin{tabular}{|c|c|c|}
\hline \multirow{4}{*}{$\begin{array}{c}\text { Reaction } \\
\text { Rate }\end{array}$} & $\begin{array}{c}\text { LEU at 12 MW rel. to } \\
\text { HEU at 10 MW }\end{array}$ \\
\cline { 2 - 3 } & $\mathrm{Be}-9(\mathrm{n}, 2 \mathrm{n})$ & $10.5 \%$ \\
\cline { 2 - 3 } & $\mathrm{Be}-9(\mathrm{n}, \alpha)$ & $9.0 \%$ \\
\cline { 2 - 3 } & $\mathrm{Li}-6(\mathrm{n}, \mathrm{T})$ & $-5.2 \%$ \\
\hline \multirow{3}{*}{ Flux } & $\mathrm{Be}-9(\gamma, 2 \alpha)$ & $-10.2 \%$ \\
\cline { 2 - 3 } & $\mathrm{E}>1.0 \mathrm{MeV}$ & $8.9 \%$ \\
\cline { 2 - 3 } & $\mathrm{E}<0.1 \mathrm{MeV}$ & $0.8 \%$ \\
\cline { 2 - 3 } & $\mathrm{E}<1.0 \mathrm{eV}$ & $-4.9 \%$ \\
\hline
\end{tabular}

The profile of helium concentration in the reflector after 8 years of operation (7.2 full-power years) with LEU at $12 \mathrm{MW}$ is presented in Figure A.26. The maximum estimated helium concentration is 4,377 appm, which is $9.3 \%$ greater than the maximum helium concentration for the reflector with the HEU core for the same operation time. The average helium concentration in the reflector at 8 years operation is also greater for the LEU core, as summarized in Table A.7. 


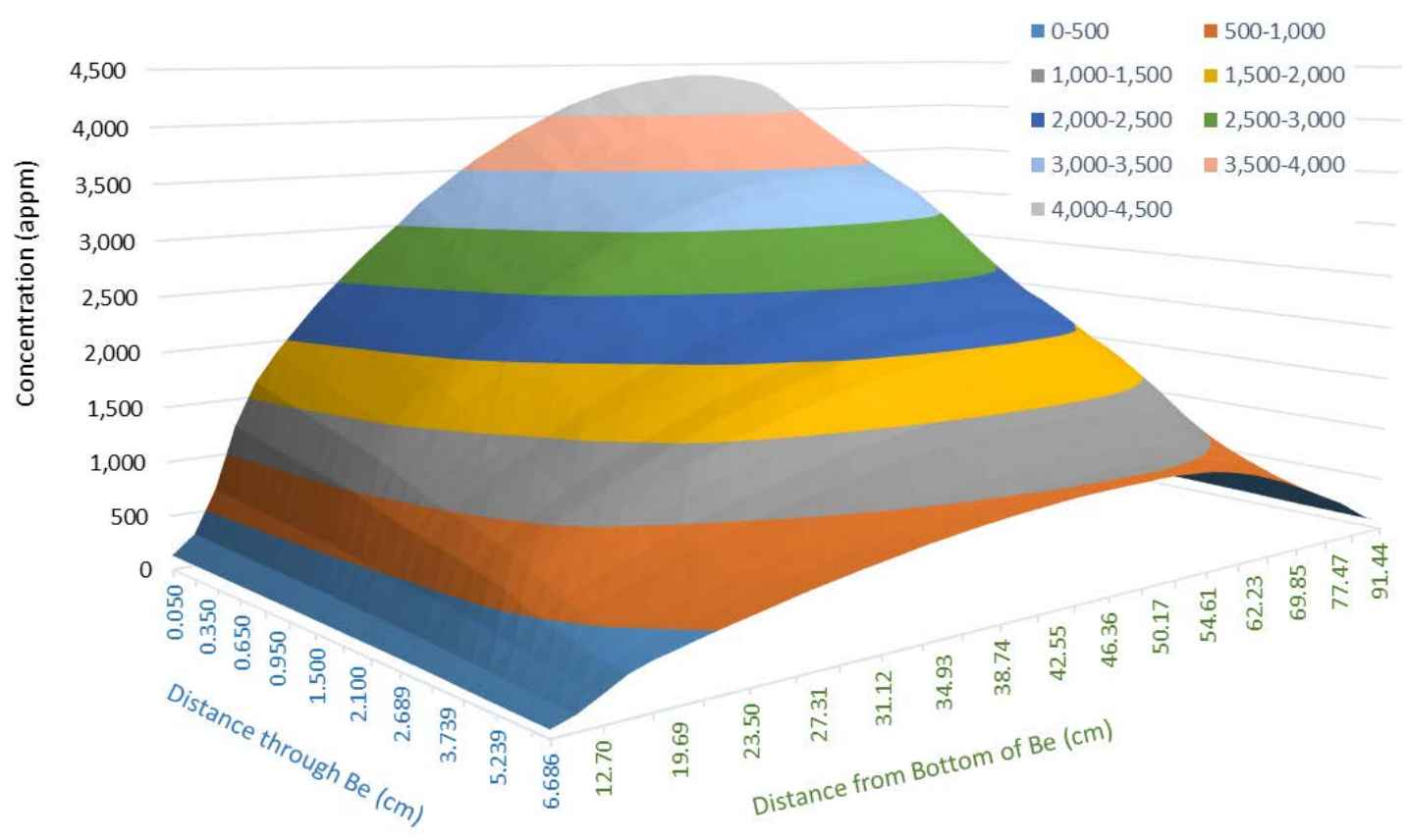

Figure A.26. Total He-4 produced in LEU beryllium reflector at 8 years of operation (LEU core at equilibrium Xe)

Table A.7. He-4 concentration (appm) in beryllium reflector

\begin{tabular}{|l|c|c|}
\hline & Maximum & Average \\
\hline HEU & 4005 & 1013 \\
\hline LEU & 4377 & 1101 \\
\hline LEU rel. to HEU & $9.3 \%$ & $8.7 \%$ \\
\hline
\end{tabular}

\section{A.9 Irradiation Damage in the Beryllium Reflector with LEU Core}

A detailed analysis of irradiation damage of the reflector with the HEU core, along with comparison to measured values presented in literature, was presented above. Predictions of irradiation damage in the reflector with the LEU core after conversion and following 8 years of operation (7.2 full-power years) has also been completed. As can be seen in Figure A.27, the reflector damage measured in dpa for a given fast fluence $(\mathrm{E}>0.1 \mathrm{MeV})$ is relatively unaffected by the conversion. The maximum damage at the end of 8 years operation with the LEU core at $12 \mathrm{MW}$, however, is $17.3 \mathrm{dpa}$, which is $10.2 \%$ greater than the maximum damage with the HEU core at 10 MW. Figure A.28 shows the damage profile in the reflector with the LEU core at 8 years operation. 


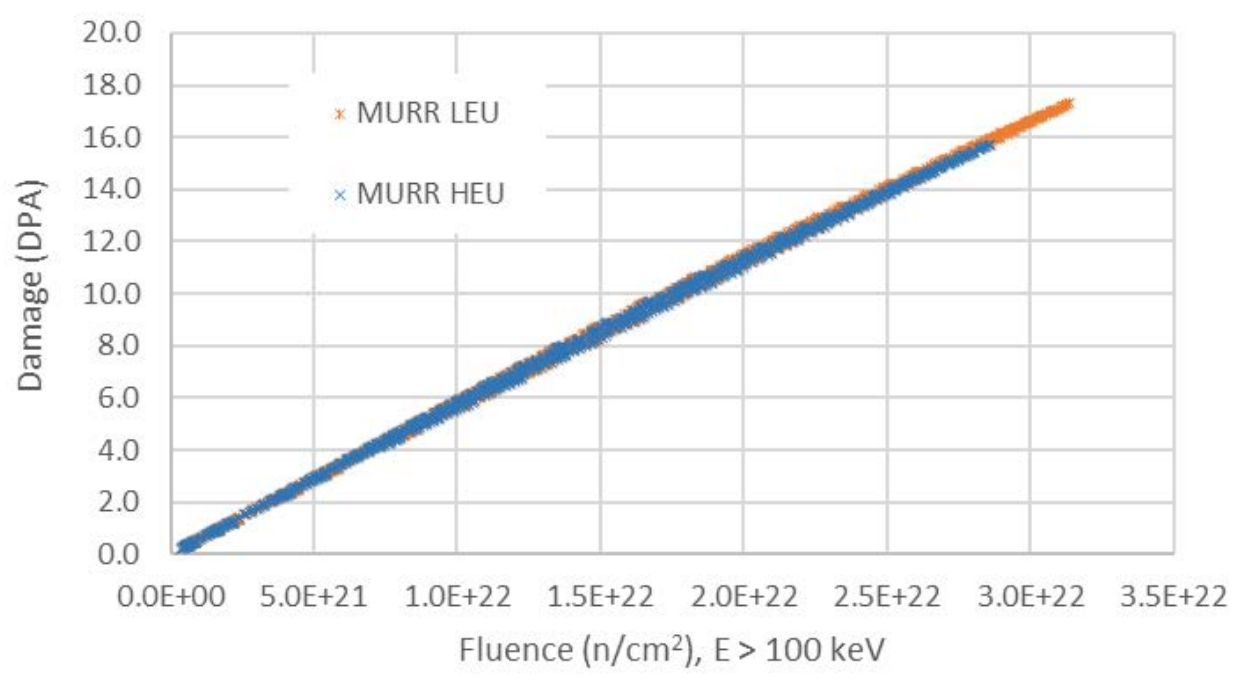

Figure A.27. Neutron damage in irradiated beryllium

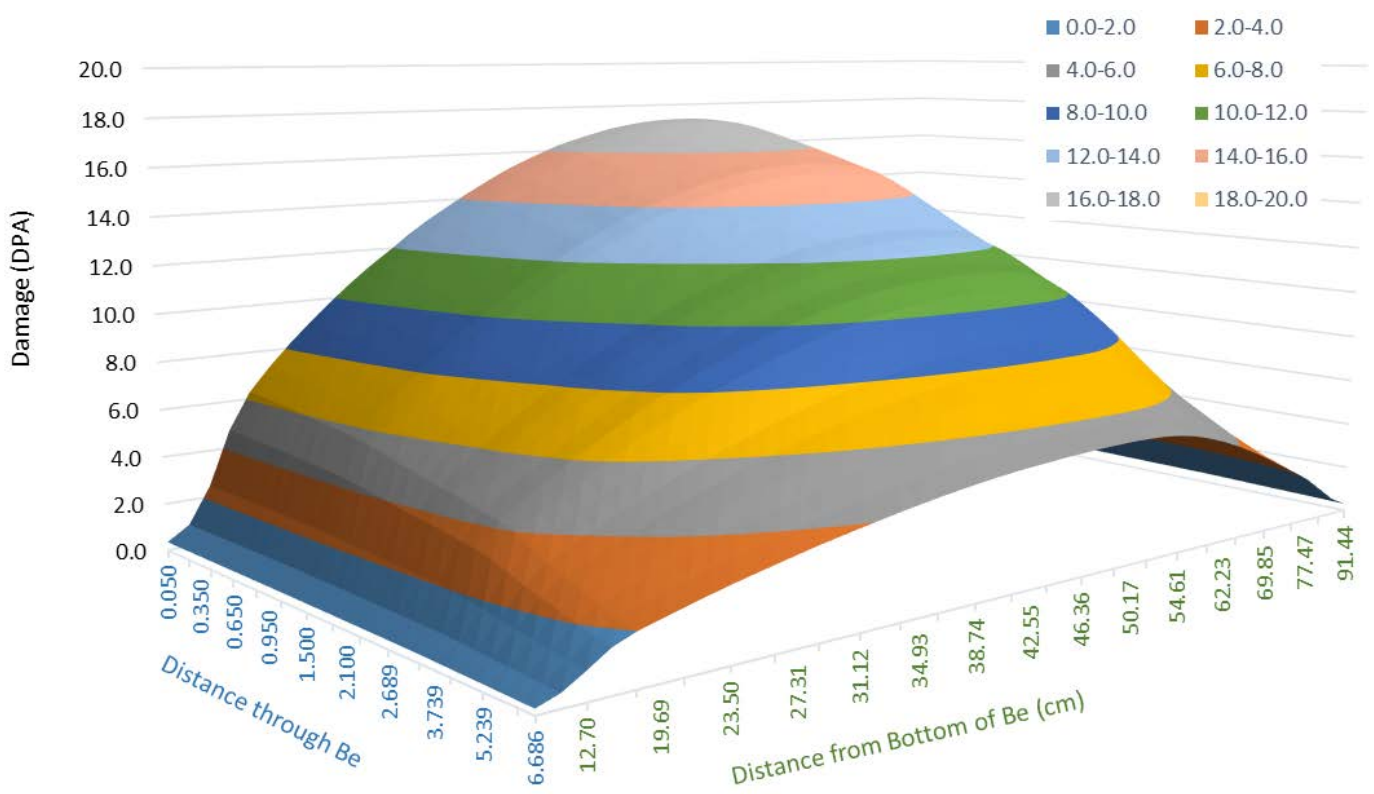

Figure A.28. Damage in beryllium reflector at 8 years of operation (LEU core at equilibrium $\mathrm{Xe)}$

\section{A.10 Neutron and Photon Heating: Beryllium Reflector with LEU Core}

Neutron and photon heating in the reflector with the LEU core was calculated using the same methodology described above for the HEU core. The average heating values from neutrons, prompt photons, and delayed photons for the reflector with the LEU and HEU core are summarized in Table A.8. The reflector heating from neutrons is $10 \%$ greater for the LEU core at $12 \mathrm{MW}$ relative to the HEU core at $10 \mathrm{MW}$ due to the increased fast neutron flux. On the other hand, the reflector heating from prompt and delayed photons is predicted to decrease by $\sim 18 \%$ following conversion, even with the 
power uprate. The roughly nine-fold increase in mass of uranium in the high-density fuel in the LEU core causes greater attenuation of the $\gamma$ flux reaching the reflector.

Table A.8. Average heating in beryllium reflector $\left(\mathrm{W} / \mathrm{cm}^{3}\right)$

\begin{tabular}{|l|c|c|}
\hline & LEU & $\begin{array}{c}\text { HEU } \\
\text { (Table A.4) }\end{array}$ \\
\hline Neutron Heating & 0.43 & 0.39 \\
\hline Prompt Photon Heating & 0.72 & 0.88 \\
\hline Est. Delayed Photon Heating & 0.65 & 0.80 \\
\hline
\end{tabular}

The total neutron and photon heating in the beryllium reflector with the LEU core is shown in Figure A.29. The peak energy deposition in the reflector with the LEU core and power uprate is predicted to be $5.11 \mathrm{~W} / \mathrm{cm}^{3}$, which is $13.4 \%$ less than for the HEU core. The total reflector heating for the reactor at $12 \mathrm{MW}$ operating power is $150 \mathrm{~kW}$, or $1.3 \%$ of the reactor power. The analysis reported in [6] also found that the reflector heating decreased with the LEU core relative to the HEU core.

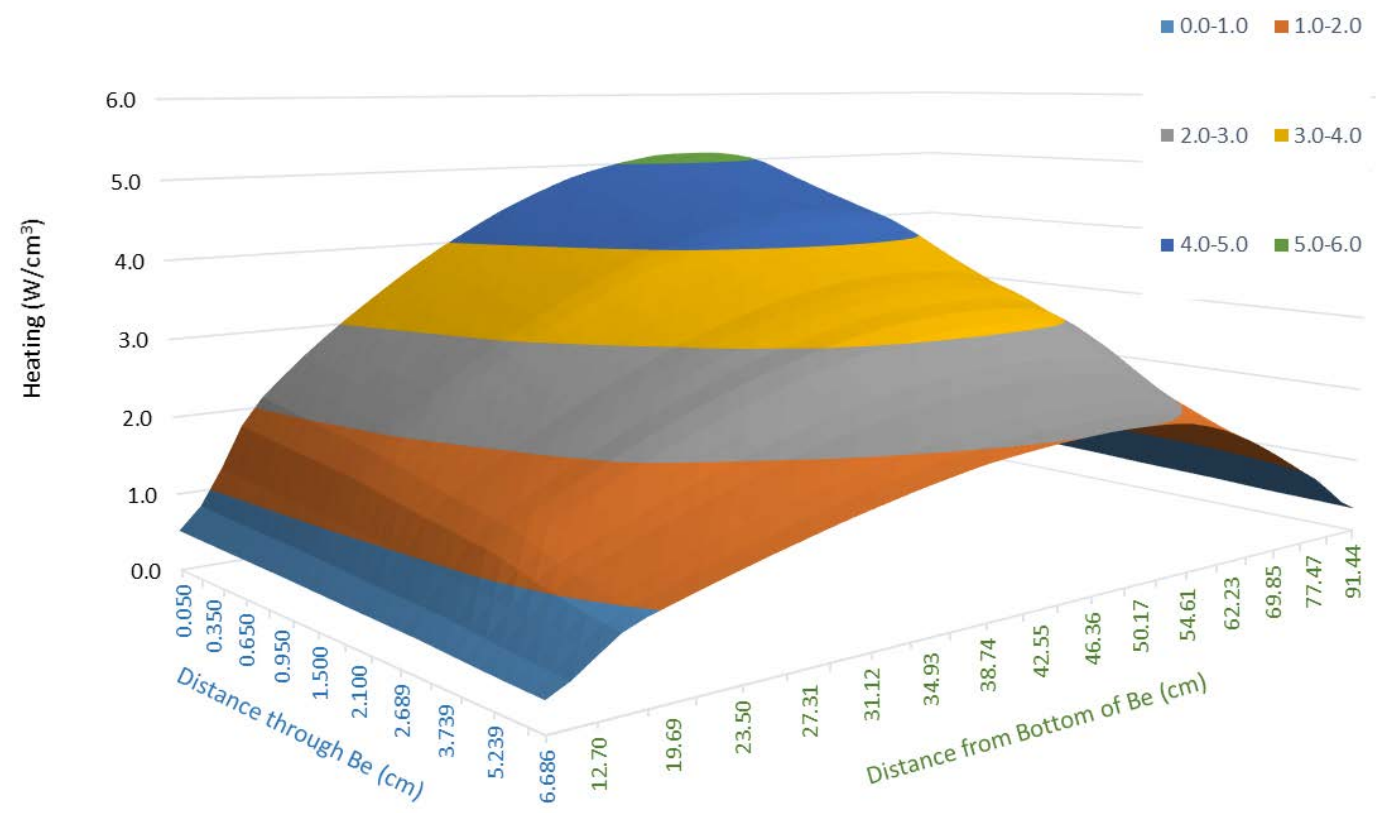

Figure A.29. Total heating in beryllium reflector (LEU core at equilibrium Xe)

\section{A.11 Azimuthal Distribution of Irradiation Conditions in Beryllium Reflector}

Using MCNP5 reaction rate tallies, the helium concentration, irradiation damage, and heating results were calculated as a function of radial and axial position in the beryllium reflector. Calculated radial and axial peak to average values were found to be $\geq 2$. Azimuthal variation of the irradiation conditions in the reflector also exist, but are expected on a much smaller numerical scale than the radial and axial variation. Consequently, tally results were homogenized azimuthally in the analysis presented above. 
As can be deduced from the reactor cross-section layout shown in Figure A.1, the irradiation conditions in the azimuthal direction for a given radial and axial position may be affected by several factors, including the variation of the burnup of the elements loaded in the core, the experimental configuration in the graphite reflector, variation in the neutron flux due to control blade burnup and presence of the regulating blade, and the impact of the grooves that are machined vertically along the inner surface of the beryllium annulus.

Structural mechanics FE analysis has found that the most likely point of failure with beryllium irradiation with both HEU and LEU cores is on the outer surface of the reflector due to a combination of increasing tensile stresses caused by swelling and thermal effects and degradation of the ultimate tensile strength of beryllium with irradiation. Beryllium failure can also occur due to compressive stresses. Although the He-4 concentration and heating are greatest on the inner surface of the reflector, the greatest compressive stress in the irradiated reflector has been found to occur at the bottom of the grooves due to stress concentration at the corners.

Figure A.30 provides a close-up view of the core and beryllium reflector. For the purpose of convenient identification in this figure, the grooves along the inner surface of the reflector are numbered one through five. A groove is located between adjacent control blades (lettered A through D) and the regulating blade. Aluminum "T-spacers" are fit into these grooves to maintain a minimum space between the outer pressure vessel and the beryllium reflector to ensure that the control blades have free, unimpinged motion.

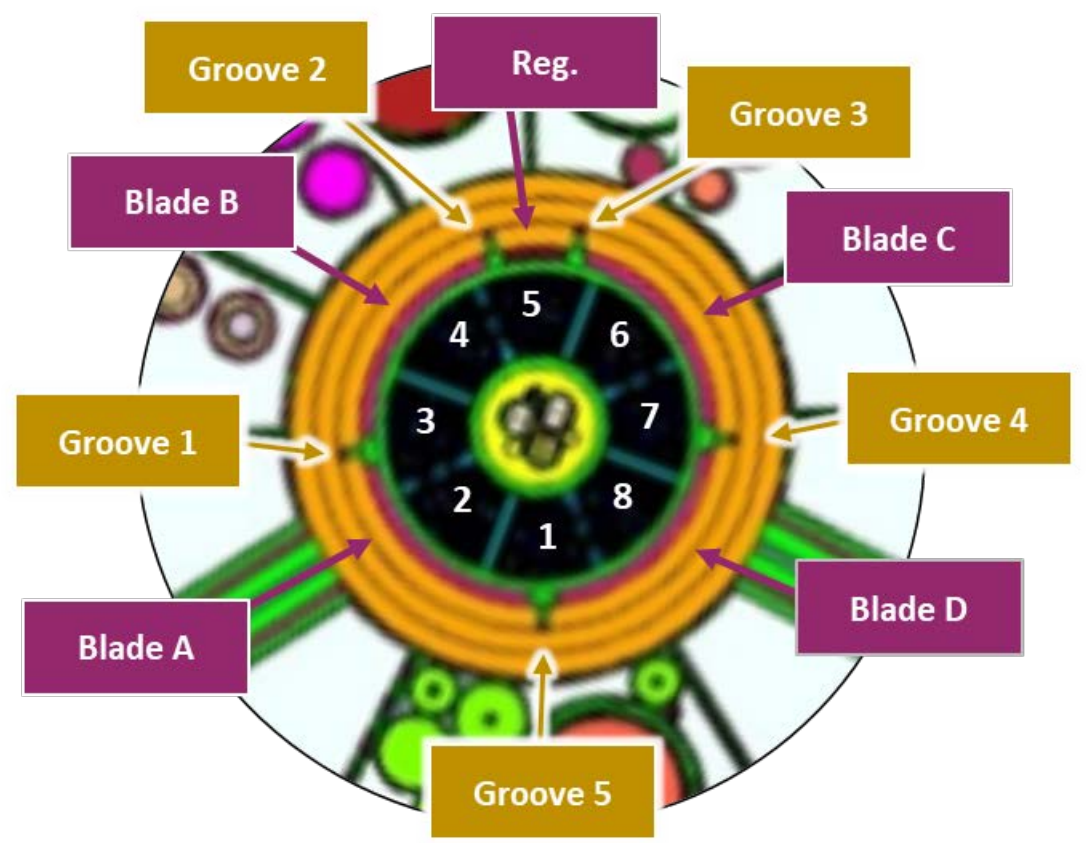

Figure A.30. Close-up view of the core and beryllium reflector

Each groove is nominally 0.375 inches wide and 0.818 inches deep, or about one-third of the total reflector thickness. The analysis presented above showed that the He-4 concentration, damage, and heating all decrease with penetration in the reflector due to attenuation of the neutron and photon flux by the beryllium material. It is expected, however, that near the bottom of the grooves the neutron and photon flux will be greater compared with azimuthally-averaged values at the same radial 
position in the reflector because the aluminum in the T-spacers is relatively transparent to neutrons and photons compared to beryllium.

Ten tally segments were included in the MCNP5 model to determine the effects of the grooves on the irradiation conditions. Five segments were defined at a position at the bottom of each groove, as shown by the yellow segment in Figure A.31. Five additional segments at the same azimuthal positions as the grooves were defined at a radial position near the outer surface of the reflector, as shown by the green segment in Figure A.31. Note the yellow and green segments in Figure A.31 are drawn only for illustrative purpose and are not to scale. The width of each tally segment was defined as the same width as the groove $(0.375$ inches). The thickness of the thin tally segments at the bottom of the grooves was defined as 0.048 inches, while the thickness of the tally segments near the outer surface of the reflector was defined as 0.197 inches. These thicknesses were determined based on existing radial mesh lines in the model.

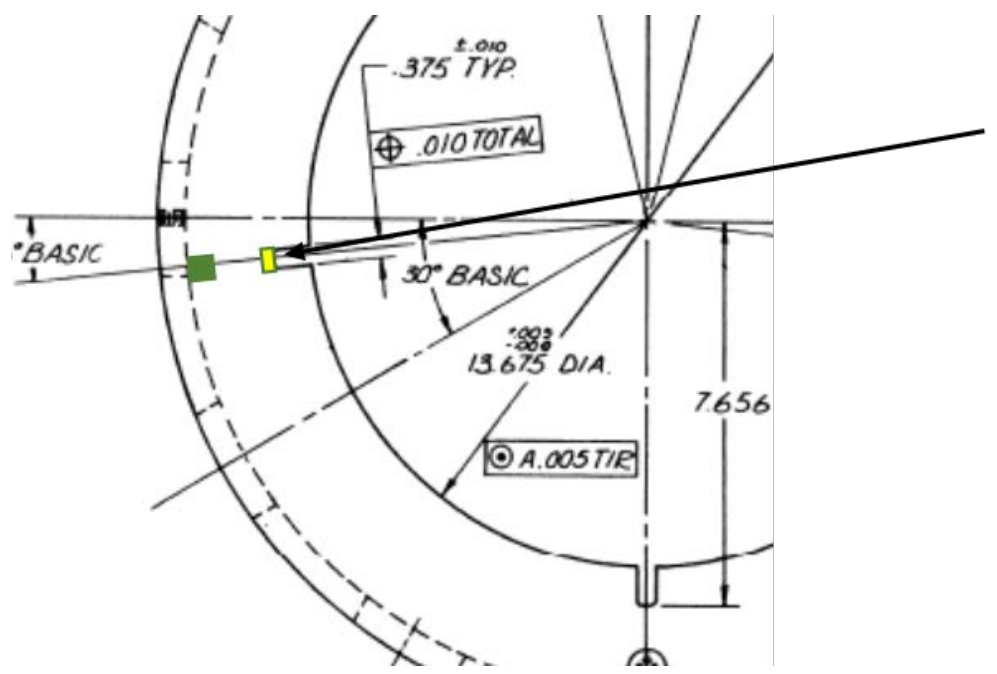

Figure A.31. MURR beryllium reflector with tally segments shown for illustrative purposes

Table A.9 provides the burnup of the elements in the MURR HEU and LEU cores under prototypic equilibrium-xenon operating conditions. The neutron and photon leakage to the beryllium reflector will be greater in azimuthal positions adjacent to elements with a larger relative power, which is expected to be those elements with a lower burnup (lower burnup leads to greater power sharing). It is expected, therefore, that the He-4 gas concentration (as well as damage and heating) will be greater near the bottom of Groove 5, which is adjacent to the lower-burnup element in core position 1, than Grooves 1 and 4, which are adjacent to the middle of life elements in core positions 3 and 7.

Table A.9. Fuel element burnup (MWd) in MURR core for prototypic operating conditions

\begin{tabular}{|c|c|c|}
\hline Element & HEU & LEU \\
\hline $\mathbf{1}$ & 3 & 3 \\
\hline $\mathbf{2}$ & 84 & 99 \\
\hline $\mathbf{3}$ & 68 & 80 \\
\hline $\mathbf{4}$ & 145 & 173 \\
\hline $\mathbf{5}$ & 3 & 3 \\
\hline $\mathbf{6}$ & 84 & 99 \\
\hline $\mathbf{7}$ & 68 & 80 \\
\hline $\mathbf{8}$ & 145 & 174 \\
\hline
\end{tabular}


Figure A.32 provides the calculated axial profile of the He-4 gas concentration in the beryllium at the bottom of each groove compared with the azimuthally-averaged concentration at the same radial position following 8 years of operation with the HEU core. Comparable results for the LEU core are provided in Figure A.33. The calculated He-4 concentration is about $9 \%$ greater at the bottom of the grooves than the azimuthally-averaged concentration at the same radial position. It can also be seen that, as expected, the He- 4 concentration is slightly greater at the bottom of groove 5 than for the other grooves because that groove is adjacent to a near fresh element. Thus, it can be expected that using the He-4 concentration and associated beryllium swelling at the bottom of the groove in the FE analysis will result in a calculated maximum compressive stress that is somewhat larger than that calculated using the azimuthally-averaged He-4 concentration at the same radial position in the reflector.

However, as stated above, the FE analysis results predict that the most likely failure location is on the outer surface of the reflector near the core mid-plane. This failure will occur when the tensile stress exceeds the irradiation-dependent ultimate tensile strength. Figure A.34 provides comparisons for the beryllium reflector with HEU and LEU cores of the axial distribution of the He-4 concentration near the outer surface of the reflector calculated at the same azimuthal position as the grooves and the azimuthally-averaged results. It can be seen from these results that effects of the grooves and fuel element burnup have no impact azimuthal distribution of the He-4 concentration on the outer surface of the reflector. The FE analysis has shown that a small fraction $(\sim 30 \%)$ of the tensile stress is due to thermal effects from neutron and photon heating. Similar to the results for the He-4 concentration, it is expected that the thermal heating on the outer surface of the reflector will be independent of azimuthal position. Consequently, the tensile stress predicted with the radially- and axiallydependent, but azimuthally-averaged, data in the FE analysis is expected to provide a reasonable estimate of the limiting stress in the beryllium reflector with irradiation.

Lastly, Figure A.35 provides comparisons of the axial distribution of the beryllium irradiation damage with HEU and LEU cores near the outer surface of the reflector. It can be seen that the damage at a given axial position is predicted to be somewhat larger $(\sim 6 \%)$ when the azimuthally-dependent tallies are used. Greater damage at the outer surface of the reflector for a given irradiation history will decrease the ultimate tensile strength of the beryllium and may decrease the time to failure. However, the effect of the azimuthal dependence of the damage is similar for both HEU and LEU cores, so the relative impact on the reflector lifetime for both cores is the same. 


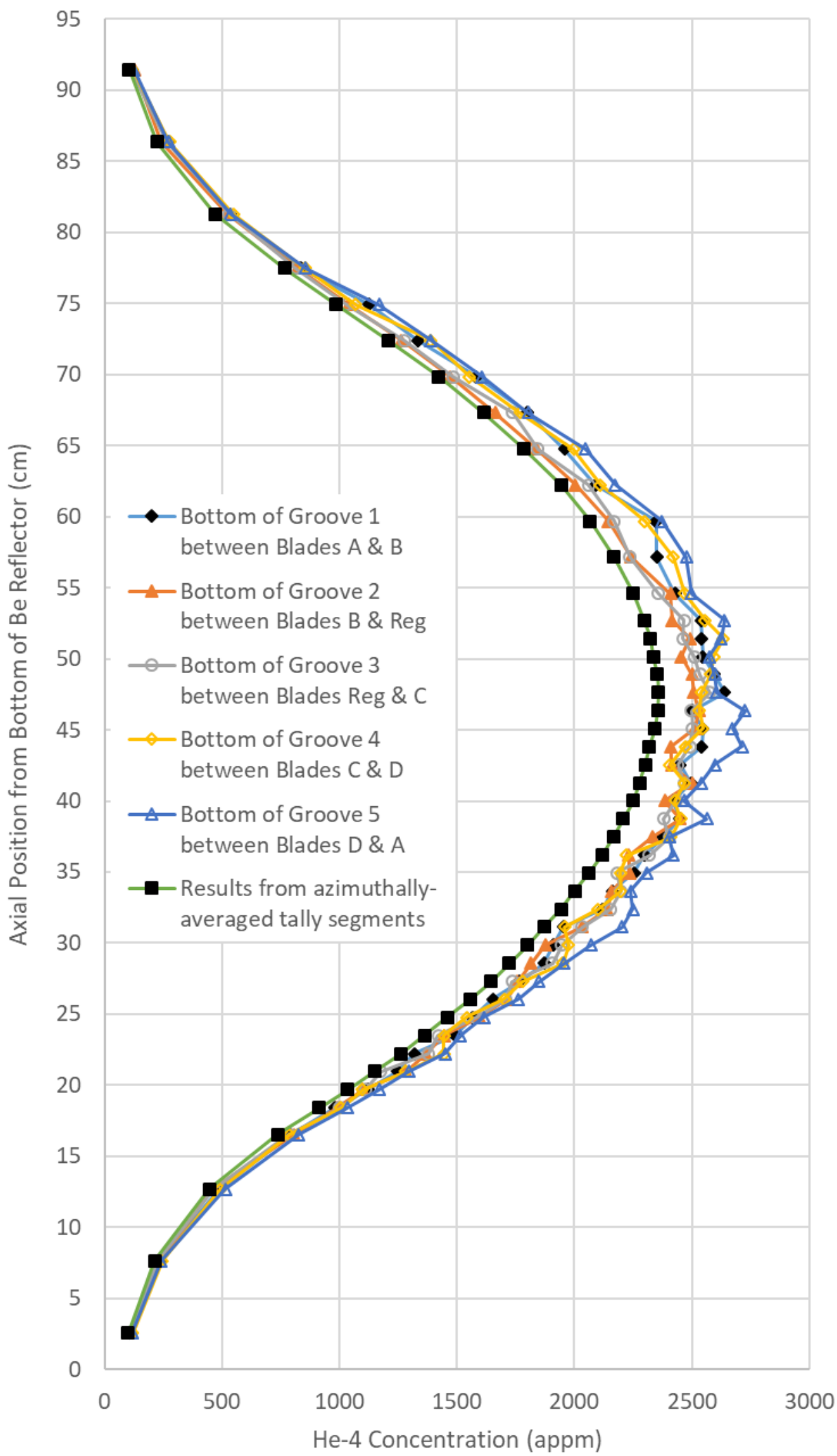

Figure A.32. Axial distribution if He-4 concentration in beryllium reflector at 8 years operation with HEU core at bottom of the grooves for T-spacers 


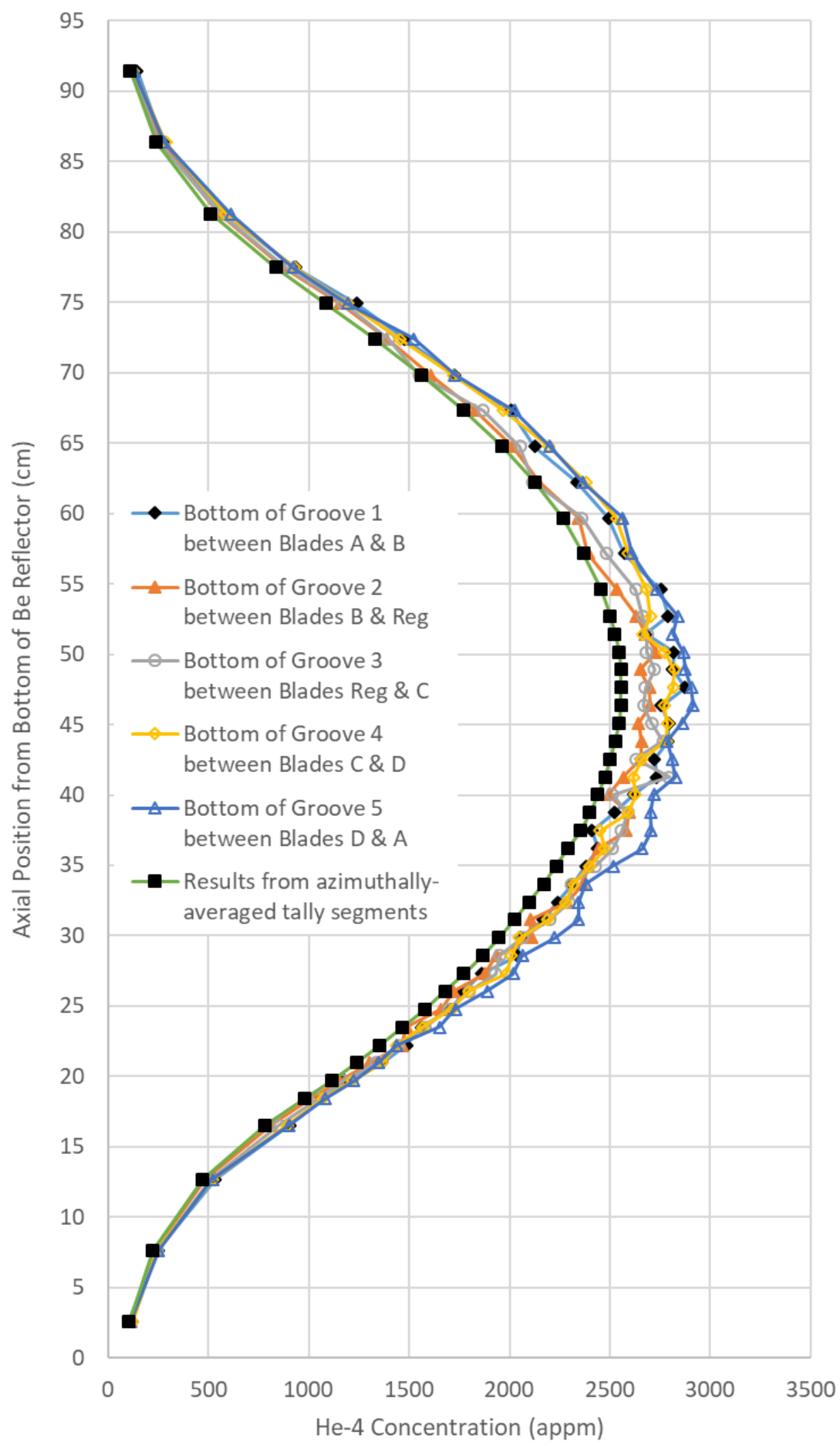

Figure A.33. Axial distribution of He-4 concentration in beryllium reflector at 8 years operation with LEU core at bottom of the grooves for T-spacers 

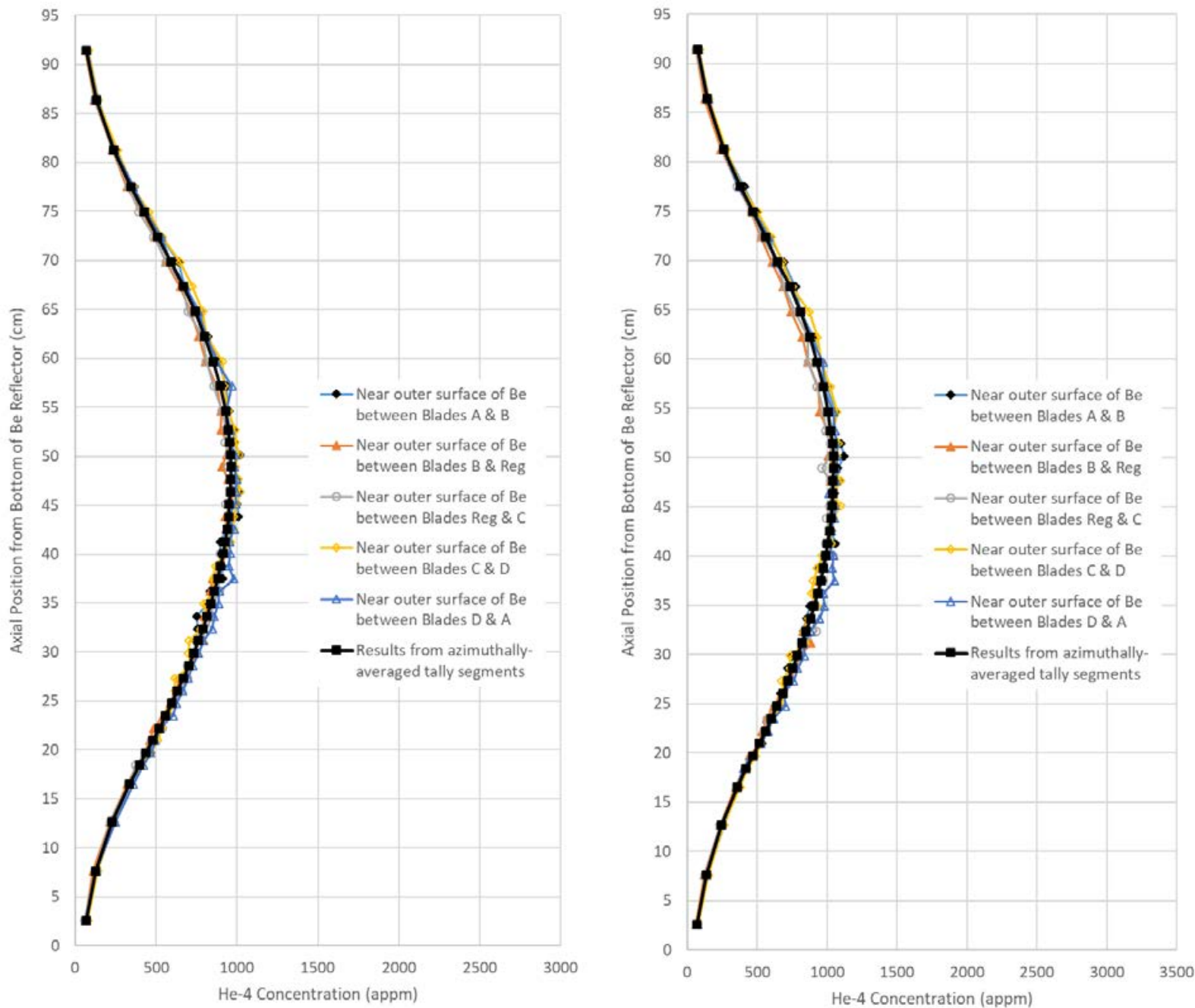

Figure A.34. Axial distribution of He-4 concentration at 8 years operation with HEU (left) and LEU (right) cores near outer surface of beryllium reflector 

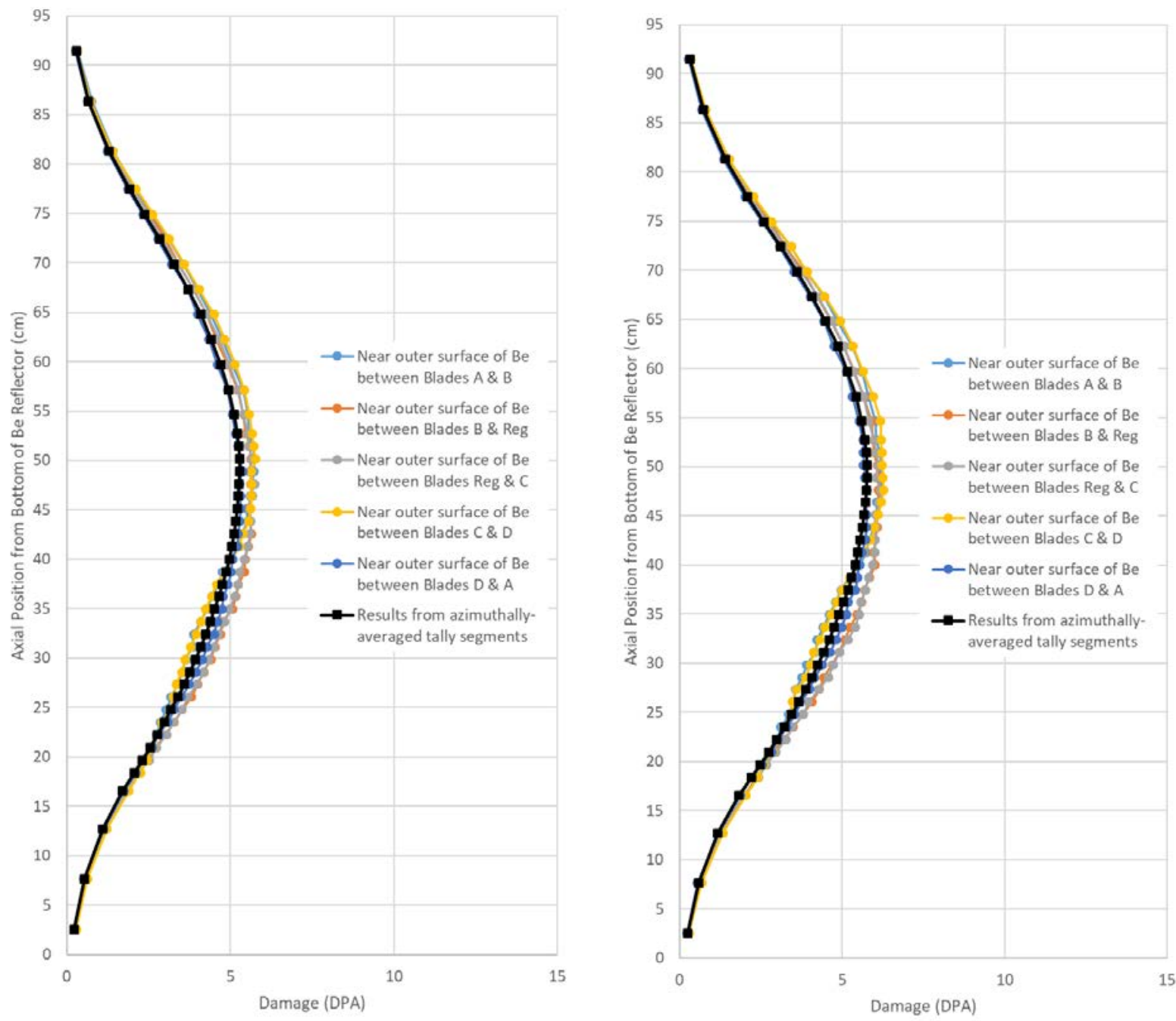

Figure A.35. Axial distribution of neutron damage (dpa) at 8 years operation with HEU (left) and LEU (right) cores near outer surface of beryllium reflector

\section{A.12 Summary}

Helium concentrations, neutron damage, and heating have been calculated for the irradiated beryllium reflector surrounding the MURR core. Tabulated results are provided in Appendix D.

The current predictions which are based on reaction rates calculated with detailed spatial discretization of the reflector using MCNP5 have indicated that the helium concentration will increase by about $9 \%$ following conversion from HEU to LEU with a $20 \%$ power uprate. Likewise, neutron damage is expected to be $10 \%$ greater for LEU with the same operating lifetime as HEU. On the other hand, total heating of the reflector from neutron and photon reactions will decrease by $13 \%$ with the LEU fuel, largely because of the greater uranium mass in the high-density monolithic LEU fuel. These are important input parameters for ongoing analyses to predict the reflector lifetime with the current HEU operations at $10 \mathrm{MW}$ and planned LEU operations at $12 \mathrm{MW}$ following conversion. 


\section{APPENDIX B: Reflector Spatial Discretization}

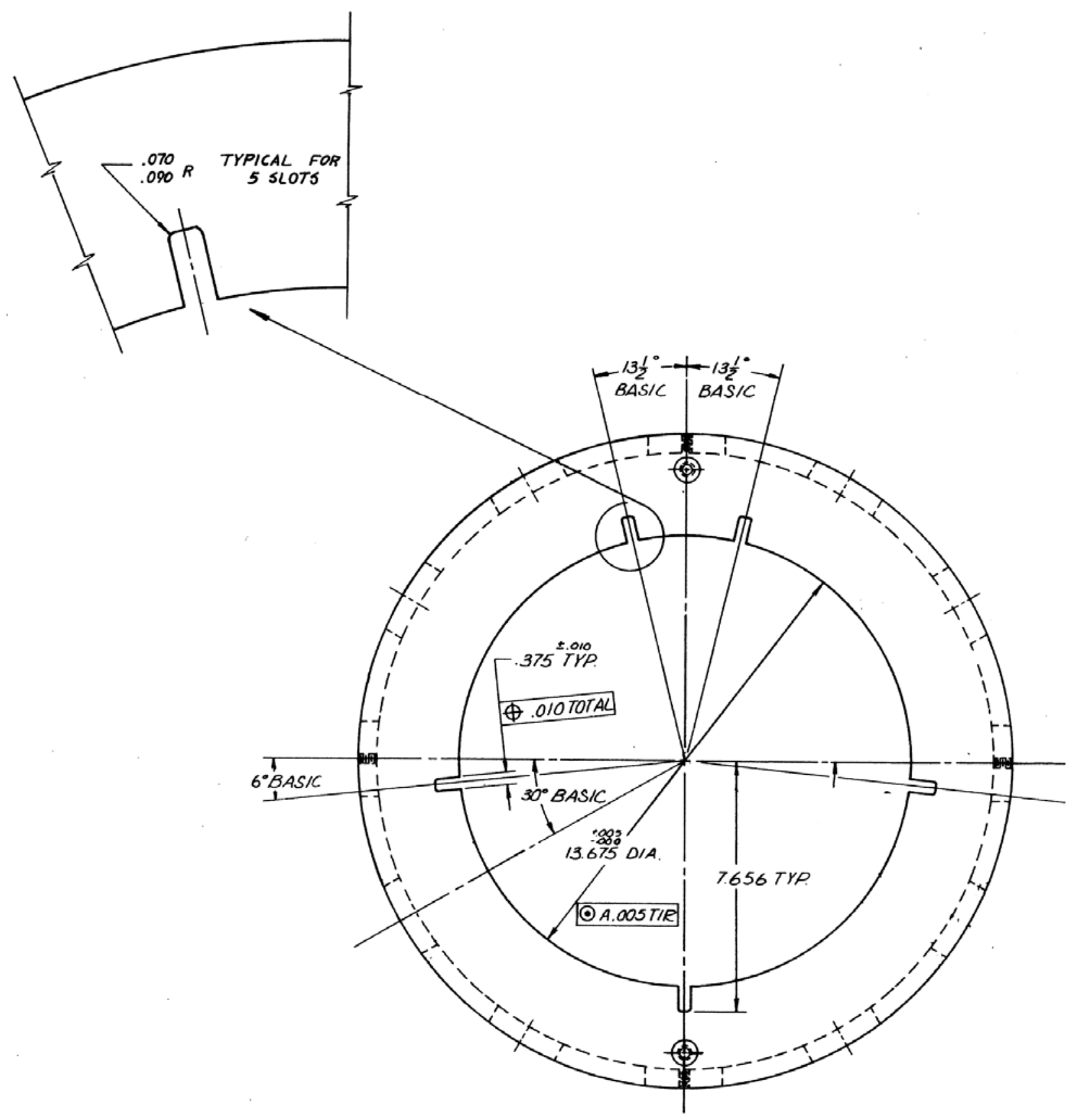

Figure B.1. MURR Beryllium Reflector Fabrication Drawing 193 (Portion of Sheet 4 of 4 showing slots in reflector for " $T$ " spacers) 
Table B.1. Axial Surfaces for Beryllium Detailed Discretization in MCNP5 Model

\begin{tabular}{|c|c|c|c|c|c|c|}
\hline \multirow{2}{*}{$\begin{array}{l}\text { Axial Level In } \\
\text { Coarse } \\
\text { Segmentation }\end{array}$} & \multirow{2}{*}{$\begin{array}{c}\text { Axial } \\
\text { Segment } \\
\text { Number }\end{array}$} & $\begin{array}{l}\text { Surf. } \\
\text { Num. }\end{array}$ & $\begin{array}{c}\text { z-value } \\
\text { (cm) }\end{array}$ & \multirow{2}{*}{$\begin{array}{c}\text { Height of } \\
\text { Axial Segment } \\
\text { (cm) }\end{array}$} & \multirow{2}{*}{$\begin{array}{c}\text { Distance from } \\
\text { bottom of } \\
\text { reflector }(\mathbf{c m})\end{array}$} & \multirow[b]{2}{*}{ MCNP Surface Card } \\
\hline & & $199^{a}$ & $-48.26^{a}$ & & & \\
\hline \multirow{6}{*}{5} & 1 & 30001 & -43.18 & 5.08 & 5.08 & $30001 \mathrm{pz}-43.18$ \\
\hline & 2 & 30002 & -38.10 & 5.08 & 10.16 & 30002 pz -38.10 \\
\hline & 3 & 30003 & -33.02 & 5.08 & 15.24 & $30003 \mathrm{pz}-33.02$ \\
\hline & 4 & 30004 & -30.48 & 2.54 & 17.78 & 30004 pz -30.48 \\
\hline & 5 & 30005 & -29.21 & 1.27 & 19.05 & 30005 pz -29.21 \\
\hline & 6 & 30006 & -27.94 & 1.27 & 20.32 & 30006 pz -27.94 \\
\hline \multirow{14}{*}{ B } & 7 & 30007 & -26.67 & 1.27 & 21.59 & $30007 \mathrm{pz}-26.67$ \\
\hline & 8 & 30008 & -25.40 & 1.27 & 22.86 & 30008 pz -25.40 \\
\hline & 9 & 30009 & -24.13 & 1.27 & 24.13 & $30009 \mathrm{pz}-24.13$ \\
\hline & 10 & 30010 & -22.86 & 1.27 & 25.40 & $30010 \mathrm{pz}-22.86$ \\
\hline & 11 & 30011 & -21.59 & 1.27 & 26.67 & $30011 \mathrm{pz}-21.59$ \\
\hline & 12 & 30012 & -20.32 & 1.27 & 27.94 & 30012 pz -20.32 \\
\hline & 13 & 30013 & -19.05 & 1.27 & 29.21 & 30013 pz -19.05 \\
\hline & 14 & 30014 & -17.78 & 1.27 & 30.48 & 30014 pz -17.78 \\
\hline & 15 & 30015 & -16.51 & 1.27 & 31.75 & $30015 \mathrm{pz}-16.51$ \\
\hline & 16 & 30016 & -15.24 & 1.27 & 33.02 & 30016 pz -15.24 \\
\hline & 17 & 30017 & -13.97 & 1.27 & 34.29 & $30017 \mathrm{pz}-13.97$ \\
\hline & 18 & 30018 & -12.70 & 1.27 & 35.56 & 30018 pz -12.70 \\
\hline & 19 & 30019 & -11.43 & 1.27 & 36.83 & 30019 pz -11.43 \\
\hline & 20 & 30020 & -10.16 & 1.27 & 38.10 & $30020 \mathrm{pz}-10.16$ \\
\hline \multirow{14}{*}{ C } & 21 & 30021 & -8.89 & 1.27 & 39.37 & 30021 pz -8.89 \\
\hline & 22 & 30022 & -7.62 & 1.27 & 40.64 & $30022 \mathrm{pz}-7.62$ \\
\hline & 23 & 30023 & -6.35 & 1.27 & 41.91 & $30023 \mathrm{pz}-6.35$ \\
\hline & 24 & 30024 & -5.08 & 1.27 & 43.18 & $30024 \mathrm{pz}-5.08$ \\
\hline & 25 & 30025 & -3.81 & 1.27 & 44.45 & $30025 \mathrm{pz}-3.81$ \\
\hline & 26 & 30026 & -2.54 & 1.27 & 45.72 & $30026 \mathrm{pz}-2.54$ \\
\hline & 27 & 30027 & -1.27 & 1.27 & 46.99 & 30027 pz -1.27 \\
\hline & 28 & 30028 & 0.00 & 1.27 & 48.26 & 30028 pz 0.00 \\
\hline & 29 & 30029 & 1.27 & 1.27 & 49.53 & 30029 pz 1.27 \\
\hline & 30 & 30030 & 2.54 & 1.27 & 50.80 & 30030 pz 2.54 \\
\hline & 31 & 30031 & 3.81 & 1.27 & 52.07 & $30031 \mathrm{pz} 3.81$ \\
\hline & 32 & 30032 & 5.08 & 1.27 & 53.34 & 30032 pz 5.08 \\
\hline & 33 & 30033 & 7.62 & 2.54 & 55.88 & 30033 pz 7.62 \\
\hline & 34 & 30034 & 10.16 & 2.54 & 58.42 & 30034 pz 10.16 \\
\hline \multirow{7}{*}{ D } & 35 & 30035 & 12.70 & 2.54 & 60.96 & $30035 \mathrm{pz} 12.70$ \\
\hline & 36 & 30036 & 15.24 & 2.54 & 63.50 & 30036 pz 15.24 \\
\hline & 37 & 30037 & 17.78 & 2.54 & 66.04 & 30037 pz 17.78 \\
\hline & 38 & 30038 & 20.32 & 2.54 & 68.58 & 30038 pz 20.32 \\
\hline & 39 & 30039 & 22.86 & 2.54 & 71.12 & 30039 pz 22.86 \\
\hline & 40 & 30040 & 25.40 & 2.54 & 73.66 & 30040 pz 25.40 \\
\hline & 41 & 30041 & 27.94 & 2.54 & 76.20 & 30041 pz 27.94 \\
\hline \multirow{4}{*}{$\mathbf{E}$} & 42 & 30042 & 30.48 & 2.54 & 78.74 & 30042 pz 30.48 \\
\hline & 43 & 30043 & 35.56 & 5.08 & 83.82 & 30043 pz 35.56 \\
\hline & 44 & 30044 & 40.64 & 5.08 & 88.90 & 30044 pz 40.64 \\
\hline & 45 & 119 & 45.72 & 5.08 & 93.98 & $119 \mathrm{pz} 45.72$ \\
\hline
\end{tabular}

a MCNP5 model surface on lower axial surface of reflector. 
Table B.2. Radial Surfaces for Beryllium Detailed Discretization in MCNP5 Model

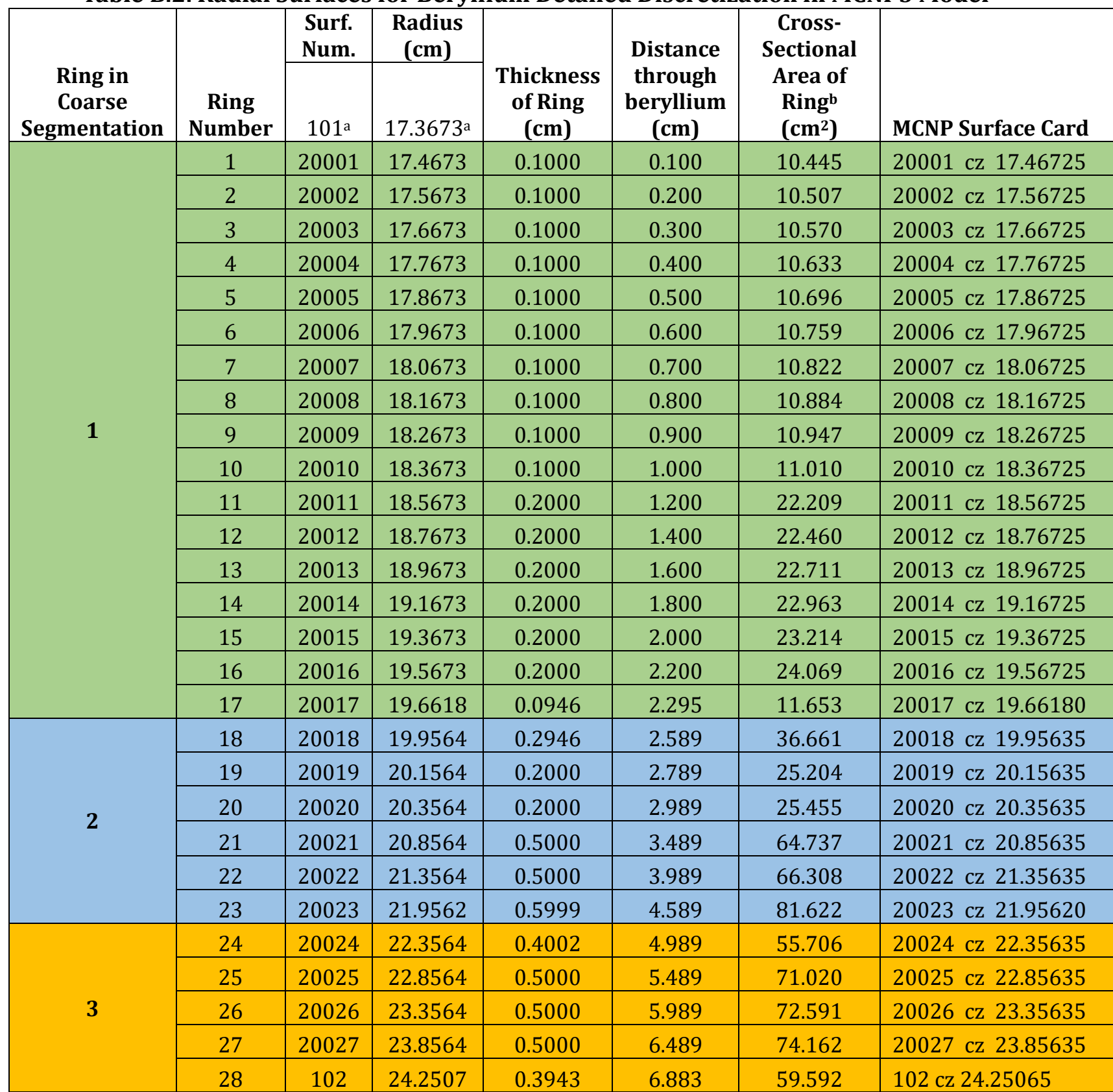

a MCNP5 model surface on inner radial surface of reflector.

${ }^{b}$ Cross-sectional area used to calculate volume of spatial zones for tally normalization. Appropriate cross-sectional area of slot cut for T-spacers has been removed from calculation for rings 1-16. Hole at the top of reflector $3.8 \mathrm{~cm}$ O.D. by $15.2 \mathrm{~cm}$ deep) for neutron source holder is included in MCNP5 model, but is not accounted for in calculating tally segment volumes used for tally normalization.

Total volume of reflector tally segments $=83,605 \mathrm{~cm}^{3}$. True reflector volume is $83,430 \mathrm{~cm}^{3}$. 


\section{APPENDIX C: Energy Discretization}

Table C.1. Energy bins for MCNP5 tallies

\begin{tabular}{|c|l|l|l|l|}
\hline Group & $\mathbf{E}_{\mathbf{m a x}}(\mathbf{M e V})$ & $\mathbf{E}_{\mathbf{m i n}}(\mathbf{M e V})$ & $\left.\mathbf{E}_{\text {avg }} \mathbf{( M e V}\right)$ & Lethargy \\
\hline $\mathbf{1}$ & $2.00 \mathrm{E}+01$ & $7.00 \mathrm{E}+00$ & $1.35 \mathrm{E}+01$ & 0.39 \\
\hline $\mathbf{2}$ & $7.00 \mathrm{E}+00$ & $5.00 \mathrm{E}+00$ & $6.00 \mathrm{E}+00$ & 1.20 \\
\hline $\mathbf{3}$ & $5.00 \mathrm{E}+00$ & $2.00 \mathrm{E}+00$ & $3.50 \mathrm{E}+00$ & 1.74 \\
\hline $\mathbf{4}$ & $2.00 \mathrm{E}+00$ & $1.00 \mathrm{E}+00$ & $1.50 \mathrm{E}+00$ & 2.59 \\
\hline $\mathbf{5}$ & $1.00 \mathrm{E}+00$ & $7.00 \mathrm{E}-01$ & $8.50 \mathrm{E}-01$ & 3.16 \\
\hline $\mathbf{6}$ & $7.00 \mathrm{E}-01$ & $5.00 \mathrm{E}-01$ & $6.00 \mathrm{E}-01$ & 3.51 \\
\hline $\mathbf{7}$ & $5.00 \mathrm{E}-01$ & $2.00 \mathrm{E}-01$ & $3.50 \mathrm{E}-01$ & 4.05 \\
\hline $\mathbf{8}$ & $2.00 \mathrm{E}-01$ & $1.00 \mathrm{E}-01$ & $1.50 \mathrm{E}-01$ & 4.89 \\
\hline $\mathbf{9}$ & $1.00 \mathrm{E}-01$ & $1.00 \mathrm{E}-02$ & $5.50 \mathrm{E}-02$ & 5.90 \\
\hline $\mathbf{1 0}$ & $1.00 \mathrm{E}-02$ & $1.00 \mathrm{E}-03$ & $5.50 \mathrm{E}-03$ & 8.20 \\
\hline $\mathbf{1 1}$ & $1.00 \mathrm{E}-03$ & $1.00 \mathrm{E}-04$ & $5.50 \mathrm{E}-04$ & 10.50 \\
\hline $\mathbf{1 2}$ & $1.00 \mathrm{E}-04$ & $1.00 \mathrm{E}-05$ & $5.50 \mathrm{E}-05$ & 12.80 \\
\hline $\mathbf{1 3}$ & $1.00 \mathrm{E}-05$ & $1.00 \mathrm{E}-06$ & $5.50 \mathrm{E}-06$ & 15.11 \\
\hline $\mathbf{1 4}$ & $1.00 \mathrm{E}-06$ & $1.00 \mathrm{E}-07$ & $5.50 \mathrm{E}-07$ & 17.41 \\
\hline $\mathbf{1 5}$ & $1.00 \mathrm{E}-07$ & $0.00 \mathrm{E}+00$ & $5.00 \mathrm{E}-08$ & 19.81 \\
\hline
\end{tabular}




\section{APPENDIX D: Helium Concentration, Neutron Damage, and Heating in Beryllium Reflector}

Table D.1. He-4 concentration in beryllium reflector with HEU core at $10 \mathrm{MW}$ over 8 years operation (appm)

\begin{tabular}{|c|c|c|c|c|c|c|c|c|c|c|c|c|c|c|c|c|c|c|c|c|c|c|c|c|c|c|c|c|c|}
\hline \multirow{2}{*}{\multicolumn{2}{|c|}{$\begin{array}{l}\text { Radial Segment } \\
\text { Distance } \\
\text { through } \\
\text { beryllium (cm) }\end{array}$}} & 1 & 2 & 3 & 4 & 5 & 6 & 7 & 8 & 9 & 10 & 11 & 12 & 13 & 14 & 15 & 16 & 17 & \begin{tabular}{|l|}
18 \\
\end{tabular} & 19 & 20 & 21 & 22 & 23 & 24 & 25 & 26 & \begin{tabular}{|l|}
27 \\
\end{tabular} & 28 \\
\hline & & 50 & 50 & 0 & 50 & 450 & 0.550 & 0.650 & 150 & 0. & 0 & 00 & 00 & 1 & 00 & 1.900 & ) & 47 & 42 & 689 & 89 & 9 & 39 & 4.289 & 789 & 39 & 739 & 239 & 36 \\
\hline \multirow{27}{*}{$\begin{array}{l}\text { Axial } \\
\text { Position } \\
\text { from } \\
\text { Bottom of } \\
\text { beryllium } \\
\text { (cm) }\end{array}$} & 91.44 & 136 & 134 & 132 & 130 & 129 & 127 & 124 & 123 & 122 & 121 & 19 & 116 & 114 & 11 & 107 & 104 & 02 & 100 & 97 & 4 & 91 & 00 & 82 & 78 & 76 & 74 & 70 & 68 \\
\hline & 86.36 & 314 & 309 & 304 & 300 & 295 & 290 & 285 & 281 & 276 & 272 & 265 & 256 & 249 & 240 & 232 & 224 & 219 & 213 & 205 & 199 & \begin{tabular}{|l|l}
189 \\
\end{tabular} & 176 & 163 & 154 & 146 & 139 & 131 & 125 \\
\hline & 81.28 & 729 & 715 & 700 & 685 & 672 & 657 & 643 & \begin{tabular}{|l|l}
630 \\
\end{tabular} & 617 & 604 & 586 & 563 & 541 & 518 & 493 & 474 & \begin{tabular}{|l|l}
460 \\
\end{tabular} & 442 & 421 & 405 & 379 & 346 & 315 & 291 & 272 & 256 & 237 & 222 \\
\hline & \begin{tabular}{|l|}
77.47 \\
\end{tabular} & 1255 & 1224 & 1192 & 1166 & 1135 & 1109 & 1080 & 1053 & 1031 & 1005 & 970 & 925 & 882 & 841 & 802 & 771 & 749 & 716 & 678 & 650 & 04 & 546 & 491 & 448 & 411 & 375 & 345 & 321 \\
\hline & 74.93 & 1648 & 1605 & 1564 & 1523 & \begin{tabular}{|l|l}
1487 \\
\end{tabular} & 1447 & 1410 & 1375 & \begin{tabular}{|l|}
1337 \\
\end{tabular} & 1304 & 1257 & 1198 & 1141 & \begin{tabular}{|l|}
1087 \\
\end{tabular} & 1039 & 995 & 962 & 920 & 868 & 828 & 768 & 692 & 617 & 558 & 512 & 466 & 426 & 394 \\
\hline & \begin{tabular}{|l|}
72.39 \\
\end{tabular} & 2039 & 1987 & 1937 & 1888 & 1837 & 1789 & 1745 & 1700 & 1654 & 1612 & 1552 & 1475 & 1403 & 1336 & 1272 & 1216 & 1177 & 1123 & 1060 & 1013 & 937 & 839 & 747 & 673 & 617 & 559 & 508 & 469 \\
\hline & 69.85 & 2410 & 2348 & 2288 & 2230 & 2168 & 2112 & 2057 & 2005 & 1955 & 1906 & 1833 & 1743 & 1657 & 1575 & 1502 & 1435 & 1385 & 1323 & 1248 & 1191 & 1101 & 984 & 876 & 789 & 719 & 653 & 594 & 545 \\
\hline & 67.31 & 2755 & 2684 & 2616 & 2546 & 480 & 2415 & 2351 & 288 & 2231 & 2174 & 090 & 1983 & 1887 & 1793 & 1710 & $\mid 1629$ & 1575 & 1502 & 1418 & 1354 & 1250 & 1117 & 991 & 893 & 814 & 738 & 671 & 617 \\
\hline & 64.77 & 3050 & 2969 & 2890 & 2815 & 2740 & 2671 & 2598 & 2531 & 2466 & 2400 & 2309 & 2196 & 2086 & 1985 & 1891 & 1803 & 1743 & 1666 & 1570 & 1499 & 1385 & 1238 & 1098 & 989 & 900 & 815 & 741 & \begin{tabular}{|l|l|}
682 \\
\end{tabular} \\
\hline & 62.23 & 3311 & 3230 & 3146 & 3062 & 2980 & 2900 & 2827 & 750 & \begin{tabular}{|l|}
2677 \\
\end{tabular} & 2607 & 2509 & \begin{tabular}{|l|}
2385 \\
\end{tabular} & 2268 & 2158 & 2053 & 1960 & 1895 & 1808 & 1705 & 1626 & 1504 & 1342 & 1191 & 1072 & 975 & 882 & 802 & 737 \\
\hline & 59.69 & 3519 & 3433 & 3342 & 3251 & 3165 & 3084 & 3002 & 922 & 2847 & 2776 & 670 & 2535 & 2412 & 2292 & 2183 & 2084 & 2014 & 1924 & 1815 & 1733 & 1598 & 1430 & 1268 & 1138 & 1038 & 938 & 853 & 784 \\
\hline & 57.15 & 3696 & 3603 & 3507 & 3413 & 3326 & 3241 & 3156 & 3071 & 2992 & 2917 & 2807 & 2664 & 2534 & 2410 & 2296 & 2189 & 2115 & 2017 & 1902 & 1817 & \begin{tabular}{|l|}
1677 \\
\end{tabular} & 1499 & 1328 & 1195 & 1090 & \begin{tabular}{|l|l}
986 \\
\end{tabular} & 896 & 823 \\
\hline & 54.61 & 3832 & 3741 & 3644 & 3548 & 3448 & 3360 & 3271 & 3186 & 3108 & \begin{tabular}{|l|}
3025 \\
\end{tabular} & 2908 & 2766 & 2629 & 2498 & 2381 & 2272 & 2194 & 2097 & 1978 & 1890 & 1746 & 1560 & 1384 & 1245 & 1134 & 1025 & 929 & 853 \\
\hline & 52.71 & 3904 & 807 & 3709 & 3610 & 3521 & 3425 & 3333 & 3246 & 3161 & 3079 & 2964 & 2824 & 2685 & 2555 & 2430 & 2316 & 2240 & 2142 & 2018 & 1925 & 1779 & 1592 & 1412 & 1268 & 1153 & 1042 & 947 & 871 \\
\hline & 51.44 & 3954 & 3852 & 3755 & 3655 & 3563 & 3470 & 3381 & 3290 & 3205 & 3114 & 2996 & 2847 & 2711 & 2578 & 2455 & 2345 & 2267 & 2163 & 2038 & 1947 & 1795 & 1602 & 1421 & 1280 & 1167 & 1053 & 953 & 874 \\
\hline & 50.17 & 3986 & 3878 & 3773 & 3677 & 3583 & 3492 & 3402 & 308 & 3229 & 3146 & 3030 & 2872 & 2730 & 2600 & 2471 & 2356 & 2279 & 2177 & 2052 & 1962 & 1807 & 1610 & 1429 & 1284 & 1170 & 1063 & 961 & 883 \\
\hline & \begin{tabular}{|l}
48.90 \\
\end{tabular} & 97 & 93 & 83 & 38 & 94 & 00 & 88 & 16 & 32 & 54 & 34 & 30 & 39 & 507 & 80 & 74 & 2293 & 84 & 64 & 70 & 15 & 1623 & 38 & 292 & 74 & 62 & 4 & 385 \\
\hline & 4 & 05 & 04 & 00 & 0 & 3602 & 11 & 3 & 32 & 1 & 6 & 43 & 2 & 53 & 611 & 86 & 77 & 2302 & 93 & 663 & 1966 & 5 & 1624 & 37 & 293 & 1179 & 63 & 51 & 82 \\
\hline & 466.36 & 4003 & 05 & 3801 & 3696 & 3601 & 3505 & 3419 & 3329 & 3244 & 3157 & 337 & 2894 & 2753 & 617 & 90 & 377 & 2298 & 88 & 2064 & 1966 & 314 & 1621 & 435 & 1292 & 1171 & 059 & 958 & 877 \\
\hline & 45.09 & 3980 & 3889 & 3789 & 3690 & 3594 & 3502 & 3409 & 3319 & 3232 & 3149 & 3027 & 2871 & 2732 & 2598 & 2472 & 2364 & 2283 & 2178 & 2052 & 1958 & 1808 & 1613 & 1429 & 1286 & 1169 & 1053 & 952 & 873 \\
\hline & \begin{tabular}{|l|l|}
43.82 \\
\end{tabular} & 3961 & 3857 & 3755 & 3655 & 3563 & 3468 & 3381 & 3292 & 3204 & 3120 & 3003 & 2857 & 2713 & 2579 & 2454 & 2339 & 2265 & 2162 & 2040 & 1945 & 1794 & 1602 & 1420 & 1275 & 1160 & 1048 & 949 & 869 \\
\hline & 42.55 & 3932 & 3836 & 3730 & 3631 & 3537 & 3454 & 3358 & 3273 & 3186 & 3101 & 2988 & 2839 & 2700 & 2563 & 2437 & 2324 & 2245 & 2142 & 2024 & 1935 & 1783 & 1592 & 408 & 1266 & 1153 & 040 & 939 & 858 \\
\hline & \begin{tabular}{|l|l|}
41.28 \\
\end{tabular} & 3882 & 3782 & 3686 & 3593 & 3496 & 3402 & 3313 & 3220 & 3143 & 3053 & 2943 & 2797 & 2660 & 2533 & 2409 & 2298 & 2220 & 2118 & 1999 & 1906 & 1760 & 1571 & 1391 & 1247 & 1135 & 1023 & 927 & 854 \\
\hline & 40.01 & 3831 & 3736 & 3645 & 3547 & 3448 & 3362 & 3274 & 3189 & 3105 & 3019 & 2901 & 2756 & 2621 & 2495 & 2377 & 2270 & 2194 & 2091 & 1970 & 1875 & 1731 & 1551 & 1373 & 1229 & 1120 & 1010 & 916 & 838 \\
\hline & \begin{tabular}{|l|}
38.74 \\
\end{tabular} & 3769 & 74 & 576 & 3485 & 3393 & 3302 & 3216 & 134 & 3054 & 2975 & 363 & 717 & 2577 & 453 & 33 & 228 & 52 & 54 & 38 & 53 & 06 & 1523 & 48 & 209 & 98 & 91 & 98 & 23 \\
\hline & \begin{tabular}{|l|l|}
37.47 \\
\end{tabular} & 3690 & 3594 & 3502 & 3406 & 3320 & 3232 & 3147 & 3063 & 2988 & 2915 & 2804 & 2665 & 2531 & 2405 & 2291 & 2185 & 2112 & 2017 & 1898 & 1812 & 1675 & 1494 & 1325 & 1189 & 1081 & 975 & 882 & 805 \\
\hline & 36.20 & 3614 & 3518 & 3429 & 3339 & 3253 & 3168 & 3080 & 2998 & 2916 & 2844 & 2734 & 2601 & 2472 & 2350 & 2237 & 2136 & 2063 & 1968 & 1855 & 1770 & 1633 & 1457 & 1289 & 1159 & 1053 & 952 & 860 & 786 \\
\hline
\end{tabular}


Table D.2. He-4 concentration in beryllium reflector with HEU core at $10 \mathrm{MW}$ over 8 years operation (appm) - continued

\begin{tabular}{|c|c|c|c|c|c|c|c|c|c|c|c|c|c|c|c|c|c|c|c|c|c|c|c|c|c|c|c|c|c|}
\hline \multirow{2}{*}{\multicolumn{2}{|c|}{ idjal $S$}} & & & & & & & & & & & & & & & & & & & & & & & & & & & & \\
\hline & & 1 & 2 & 3 & 4 & 5 & 6 & 7 & 8 & 9 & 10 & 11 & 12 & 13 & 14 & 15 & 16 & 17 & 18 & 19 & 20 & 21 & 22 & 23 & 24 & 25 & 26 & 27 & \begin{tabular}{|l|}
28 \\
\end{tabular} \\
\hline \multicolumn{2}{|c|}{$\begin{array}{l}\text { Distance } \\
\text { through } \\
\text { beryllium }(\mathrm{cm})\end{array}$} & 0.05 & \begin{tabular}{|l|}
0.15 \\
0
\end{tabular} & 0.25 & \begin{tabular}{|l|}
0.35 \\
0
\end{tabular} & $\begin{array}{l}0.45 \\
0\end{array}$ & 0.55 & \begin{tabular}{|l|}
0.65 \\
0
\end{tabular} & 0.75 & \begin{tabular}{|l|}
0.85 \\
0
\end{tabular} & \begin{tabular}{|l|}
0.95 \\
0
\end{tabular} & $\begin{array}{l}1.10 \\
0\end{array}$ & 30 & $\begin{array}{l}1.50 \\
0\end{array}$ & 1.70 & 1.90 & $\begin{array}{l}2.10 \\
0\end{array}$ & $\begin{array}{l}2.24 \\
7\end{array}$ & $\begin{array}{l}2.44 \\
2\end{array}$ & $\begin{array}{l}2.68 \\
9\end{array}$ & \begin{tabular}{|l}
2.88 \\
9
\end{tabular} & $\begin{array}{l}3.23 \\
9\end{array}$ & $\begin{array}{l}3.73 \\
9 \\
\end{array}$ & $\begin{array}{l}4.28 \\
9\end{array}$ & \begin{tabular}{|l|}
4.78 \\
9
\end{tabular} & \begin{tabular}{|l|}
5.23 \\
9
\end{tabular} & $\begin{array}{l}5.73 \\
9\end{array}$ & $\begin{array}{l}6.23 \\
9 \\
\end{array}$ & \begin{tabular}{|l}
6.68 \\
6
\end{tabular} \\
\hline \multirow{18}{*}{\begin{tabular}{|l|} 
Axial \\
Position \\
from \\
Bottom of \\
berylliu \\
$\mathrm{m}(\mathrm{cm})$
\end{tabular}} & \begin{tabular}{|l|l|}
34.9 \\
3 \\
\end{tabular} & 15 & 3428 & 3338 & 3249 & 3165 & 3078 & 2997 & 2926 & 2849 & 2773 & 2668 & 2536 & 2413 & 2292 & 2183 & 2082 & 2012 & 1922 & 1813 & 1728 & 1593 & 1423 & 1259 & 1131 & 1028 & 929 & 839 & 768 \\
\hline & \begin{tabular}{|l|l|}
33.6 \\
6 \\
\end{tabular} & 3427 & 3339 & 3253 & 3168 & 3081 & 3002 & 2928 & 2851 & 2772 & 2696 & 2598 & 2469 & 2343 & 2228 & 2118 & 2019 & 1954 & 1865 & 1760 & 1679 & 1550 & 1382 & 1225 & 1095 & 998 & 901 & 814 & 745 \\
\hline & \begin{tabular}{|l|}
32.3 \\
9 \\
\end{tabular} & 3320 & 3234 & 3152 & 3063 & 2980 & 2905 & 2830 & 2757 & 2684 & 2614 & 2509 & 2383 & 2261 & 2153 & 2053 & 1960 & 1893 & 1804 & 1701 & 1622 & 1494 & 1333 & 1183 & 1063 & 966 & 871 & 789 & 721 \\
\hline & \begin{tabular}{|l|}
31.1 \\
2 \\
\end{tabular} & 3193 & 3110 & 3030 & 2951 & 2872 & 2804 & 2728 & 2658 & 2588 & 2526 & 2425 & 2306 & 2191 & 2084 & 1982 & 1886 & 1828 & 1743 & 1641 & 1569 & 1445 & 1289 & 1141 & 1025 & 933 & 841 & 760 & 696 \\
\hline & \begin{tabular}{|l|l|}
29.8 \\
5 \\
\end{tabular} & 3081 & 3000 & 2923 & 2843 & 2771 & 2691 & 2620 & 2552 & 2485 & 2419 & 2322 & 2213 & 2104 & 1999 & 1903 & 1817 & 1757 & 1679 & 1582 & 1509 & 1394 & 1244 & 1099 & 986 & 897 & 812 & 733 & 670 \\
\hline & \begin{tabular}{|l|}
28.5 \\
8 \\
\end{tabular} & 2944 & 2865 & 2790 & 2720 & 2648 & 2579 & 2515 & 2451 & 2383 & 2326 & 2232 & 2120 & 2014 & 1919 & 1825 & 1739 & 1677 & 1604 & 1513 & 1443 & 1331 & 1187 & 1052 & 946 & 861 & 776 & 701 & 642 \\
\hline & \begin{tabular}{|l|}
27.3 \\
1 \\
\end{tabular} & 2803 & 2733 & 2660 & 2592 & 2523 & 2452 & 2392 & 2330 & 2272 & 2215 & 2130 & 2022 & 1922 & 1830 & 1744 & 1660 & 1606 & 1529 & 1441 & 1376 & 1269 & 1132 & 1001 & 897 & 815 & 737 & 669 & 611 \\
\hline & \begin{tabular}{|l}
26.0 \\
4 \\
\end{tabular} & 2651 & 2584 & 2520 & 2451 & 2388 & 2325 & 2262 & 2205 & 2146 & 2092 & 2013 & 1910 & 1815 & 1724 & 1643 & 1572 & 1521 & 1450 & 1365 & 1301 & 1197 & 1071 & 950 & 853 & 775 & 698 & 631 & 578 \\
\hline & \begin{tabular}{|l|}
24.7 \\
7 \\
\end{tabular} & 2498 & 2432 & 2368 & 2309 & 2244 & 2184 & 2127 & 2074 & 2020 & 1966 & 1893 & 1800 & 1711 & 1627 & 1544 & 1472 & 1423 & 1363 & 1282 & 1223 & 1128 & 1004 & 889 & 800 & 729 & 659 & 598 & 547 \\
\hline & \begin{tabular}{|l|}
23.5 \\
0 \\
\end{tabular} & 2335 & 2271 & 2214 & 2157 & 2101 & 2041 & 86 & 1934 & 1884 & 1835 & 1768 & 1679 & 1596 & 1513 & 1439 & 1374 & 1329 & 1270 & 1196 & 1142 & 1055 & 943 & 836 & 48 & 80 & 616 & 559 & 515 \\
\hline & \begin{tabular}{|l|}
22.2 \\
3 \\
\end{tabular} & 2167 & 2113 & 55 & 1999 & 1944 & 91 & 39 & 1795 & 45 & 1694 & 1632 & 1552 & 1474 & 1401 & 1332 & 1273 & 1231 & 1175 & 1107 & 1055 & 974 & 873 & 773 & 696 & 35 & 573 & 519 & 478 \\
\hline & \begin{tabular}{|l|}
20.9 \\
6 \\
\end{tabular} & 1969 & 18 & 1868 & 1817 & 67 & 19 & 72 & 1628 & 84 & 44 & 1485 & 13 & 44 & 279 & 1215 & 59 & 20 & 071 & 1011 & 54 & 93 & 97 & 708 & 38 & 83 & 530 & 479 & 440 \\
\hline & \begin{tabular}{|l|}
19.6 \\
9 \\
\end{tabular} & 1758 & 07 & 1664 & 1619 & 79 & 39 & 98 & 1461 & 20 & 83 & 1330 & 1263 & 01 & 47 & 1092 & 1043 & 09 & 963 & 14 & 33 & 803 & 21 & 642 & 82 & 31 & 483 & 439 & 405 \\
\hline & \begin{tabular}{|l|}
18.4 \\
2 \\
\end{tabular} & 1525 & 1491 & 1453 & 1420 & 1382 & 1345 & 1308 & 1273 & 43 & 1209 & 1166 & 1112 & 61 & 10 & 963 & 1 & 892 & 854 & 308 & 73 & 717 & 647 & 575 & 521 & 78 & 434 & 397 & 366 \\
\hline & \begin{tabular}{|l}
16.5 \\
1 \\
\end{tabular} & 1171 & 1144 & 1118 & 1092 & 1067 & 1042 & 1018 & 996 & 974 & 951 & 9 & 30 & 1 & 804 & 771 & 2 & 719 & 689 & 53 & 27 & 586 & 31 & 476 & 433 & 99 & 365 & 334 & 309 \\
\hline & \begin{tabular}{|l}
12.7 \\
0 \\
\end{tabular} & 646 & 634 & 624 & 612 & 601 & 589 & 578 & 568 & 58 & 548 & 534 & 14 & 496 & 478 & 62 & 448 & 437 & 423 & 405 & 391 & 369 & 339 & 309 & 285 & 265 & 246 & 227 & 212 \\
\hline & 7.62 & 284 & 282 & 278 & 273 & 269 & 266 & 263 & 258 & 254 & 251 & 246 & 38 & 32 & 2 & 19 & 214 & 210 & 20 & 197 & 191 & 183 & 173 & 161 & 151 & 143 & 134 & 127 & 120 \\
\hline & 2.54 & 126 & 124 & 123 & 121 & 120 & 119 & 117 & 116 & 1114 & 114 & 112 & 10 & 107 & 105 & 103 & 101 & 90 & a & 95 & 92 & 85 & 86 & 81 & p & 74 & 70 & 67 & 64 \\
\hline
\end{tabular}


Table D.3. Helium concentration in beryllium reflector with LEU core at $12 \mathrm{MW}$ over 8 years operation (appm)

\begin{tabular}{|c|c|c|c|c|c|c|c|c|c|c|c|c|c|c|c|c|c|c|c|c|c|c|c|c|c|c|c|c|c|}
\hline \multicolumn{2}{|c|}{\begin{tabular}{|l|} 
Radial Segment \\
\end{tabular}} & \multirow{2}{*}{\begin{tabular}{|l|}
$\mathbf{1}$ \\
0.050 \\
\end{tabular}} & \multirow{2}{*}{\begin{tabular}{|l|}
2 \\
0.150
\end{tabular}} & \multirow{2}{*}{\begin{tabular}{|l|l|}
3 \\
0.250
\end{tabular}} & \multirow{2}{*}{\begin{tabular}{|l|}
4 \\
0.350 \\
\end{tabular}} & \multirow{2}{*}{\begin{tabular}{|l|}
$\mathbf{5}$ \\
0.450 \\
\end{tabular}} & \multirow{2}{*}{\begin{tabular}{|l|}
6 \\
0.550 \\
\end{tabular}} & \multirow{2}{*}{\begin{tabular}{|l|}
7 \\
0.650
\end{tabular}} & \multirow{2}{*}{\begin{tabular}{|l|}
8 \\
0.750 \\
\end{tabular}} & & \multirow{2}{*}{\begin{tabular}{|l|}
10 \\
0.950 \\
\end{tabular}} & \multirow{2}{*}{\begin{tabular}{|l|}
11 \\
1.100
\end{tabular}} & \multirow{2}{*}{\begin{tabular}{|l|}
12 \\
1.300 \\
\end{tabular}} & \multirow{2}{*}{\begin{tabular}{|l|}
13 \\
1.500 \\
\end{tabular}} & \multirow{2}{*}{\begin{tabular}{|l|}
14 \\
1.700 \\
\end{tabular}} & \multirow{2}{*}{\begin{tabular}{|l|}
15 \\
1.900 \\
\end{tabular}} & \multirow{2}{*}{\begin{tabular}{|l|}
16 \\
2.100 \\
\end{tabular}} & \multirow{2}{*}{\begin{tabular}{|l|}
17 \\
2.247 \\
\end{tabular}} & \multirow{2}{*}{\begin{tabular}{|l|}
$\mathbf{1 8}$ \\
2.442 \\
\end{tabular}} & \multirow{2}{*}{\begin{tabular}{|l|}
19 \\
2.689 \\
\end{tabular}} & 20 & 21 & 22 & 23 & 24 & 25 & 26 & 27 & 28 \\
\hline & $\begin{array}{l}\text { through } \\
\text { (cm) }\end{array}$ & & & & & & & & & & & & & & & & & & & & 2.889 & 3.239 & 39 & 4.289 & 4.789 & .239 & 5.739 & 6.239 & 6.686 \\
\hline & 91.44 & 146 & 145 & 142 & 139 & 137 & 137 & 136 & 134 & 132 & 130 & 128 & 125 & 121 & 118 & 115 & 113 & 111 & 08 & 105 & 02 & 98 & 93 & 8 & d & 81 & 79 & 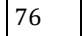 & 4 \\
\hline & 86.36 & 38 & 335 & 329 & 325 & 319 & 314 & 309 & 5 & 299 & 296 & 289 & 279 & 271 & 262 & 251 & 2 & 238 & 231 & 222 & 216 & 204 & 90 & 177 & 166 & 59 & 52 & 43 & 136 \\
\hline & 81.28 & 96 & 781 & 764 & 50 & 733 & 18 & 3 & 688 & 71 & 60 & 540 & 14 & 0 & 565 & 37 & 515 & 501 & 482 & 59 & 441 & 413 & 377 & 343 & 318 & 298 & 79 & 58 & 242 \\
\hline & 77.47 & 1384 & 1353 & 1316 & 1280 & 1250 & 1216 & 1186 & 1159 & 1130 & 1104 & 666 & 1015 & 968 & 924 & 882 & 845 & 819 & 783 & 44 & 712 & 662 & 99 & 539 & 491 & 451 & 11 & 78 & 351 \\
\hline & 74.93 & 1827 & 1783 & 1736 & 1691 & 1647 & 1603 & 1563 & 1522 & 1481 & 1445 & 1392 & 1323 & 1260 & 1198 & 1145 & 1096 & 1058 & 1013 & 957 & 917 & 847 & 761 & 679 & 614 & 562 & 12 & 468 & 431 \\
\hline & 72.39 & 2259 & 2200 & 2143 & 2086 & 2034 & 1981 & 1924 & 1874 & 1829 & 1784 & 714 & 1633 & 1553 & 1482 & 1408 & 1342 & 1296 & 1239 & 1169 & 1117 & 1034 & 925 & 822 & 742 & 677 & 13 & 559 & 515 \\
\hline & 69.85 & 2665 & 2596 & 2529 & 2460 & 2396 & 2333 & 2270 & 2209 & 2154 & 2099 & 2016 & 1912 & 1822 & 1736 & 1653 & 1576 & 1522 & 1451 & 1370 & 1309 & 1207 & 1079 & 959 & 864 & 787 & 712 & 647 & 596 \\
\hline & 67.31 & 3033 & 2954 & 2877 & 2800 & 2727 & 2659 & 2589 & 2519 & 2455 & 2389 & 2295 & 2178 & 2071 & 1971 & 1878 & 1790 & 1731 & 1652 & 1558 & 1488 & 1375 & 1228 & 1089 & 977 & 892 & 808 & 734 & 676 \\
\hline & 64.77 & 3356 & 3274 & 3188 & 3107 & 3024 & 2943 & 2862 & 2784 & 2712 & 2638 & 2536 & 2408 & 2292 & 2183 & 2079 & 1981 & 1916 & 1827 & 1723 & 1644 & 1516 & 1356 & 1203 & 1081 & 984 & 890 & 807 & 741 \\
\hline & \begin{tabular}{|l}
62.23 \\
\end{tabular} & 3631 & 3539 & 3444 & 349 & 3263 & 3178 & 3095 & 3010 & 935 & 2863 & 2753 & 2613 & 2484 & 2361 & 2248 & 2146 & 2074 & 1979 & 1867 & 1781 & 1641 & 1465 & 1301 & 1171 & 1068 & 64 & 874 & 804 \\
\hline & 59.69 & 64 & 66 & 3668 & 571 & 79 & 386 & 3294 & 209 & 126 & 3048 & 29 & 2786 & 2647 & 2520 & 2399 & 2287 & 2209 & 2109 & 987 & 1894 & 1748 & 558 & 383 & 1244 & 134 & 025 & 929 & 854 \\
\hline & 57.15 & 48 & 46 & 3844 & 742 & 43 & 45 & 3455 & 364 & 277 & 3192 & 070 & 2915 & 2769 & 2633 & 2500 & 390 & 2311 & 2205 & 078 & 984 & 34 & 639 & 1452 & 05 & 187 & 075 & 74 & 95 \\
\hline & 54.61 & 198 & 86 & 3984 & 382 & 78 & 678 & 3583 & 483 & 391 & 3307 & 178 & 3017 & 2866 & 2726 & 2597 & 2478 & 2396 & 2285 & 154 & 2056 & 1897 & 695 & 502 & 1350 & 230 & 112 & 1007 & 20 \\
\hline & 52.7 & os & 71 & 4061 & 3950 & 849 & 43 & 41 & 41 & 3 & 70 & 46 & 3 & 2927 & 2778 & 41 & 24 & 40 & 77 & 89 & 6 & 5 & 28 & 1532 & 8 & 54 & 129 & 25 & 941 \\
\hline & 5 & & 213 & 98 & 9 & 883 & 35 & 9 & 82 & 3490 & 01 & 274 & & 52 & 2810 & 7 & 44 & 4 & 52 & 18 & 4 & 1 & 42 & 66 & 38 & 63 & 142 & 9 & 955 \\
\hline & 50.17 & 4356 & 47 & 4135 & 022 & 18 & 3823 & 3720 & 3615 & 3518 & 3432 & 3298 & 3137 & 2981 & 2837 & 2692 & 669 & 2481 & 2369 & 2231 & 2128 & 1963 & 1756 & 1555 & 1397 & 275 & 1152 & 1043 & 960 \\
\hline & 48.9 & 76 & 68 & 4152 & 42 & 36 & 28 & 23 & 28 & 35 & 3441 & 3307 & 39 & 2981 & 2839 & 33 & 80 & 2490 & 2378 & 250 & 148 & 1979 & 67 & 1564 & 1404 & 275 & 152 & 1045 & 956 \\
\hline & 47.63 & 4377 & 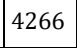 & 4 & 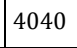 & 3937 & 3 & 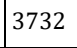 & 5 & 10 & 1 & 0 & 0 & 3 & 4 & 2 & 8 & 2 & 8 & 40 & 3 & 76 & 66 & 62 & 02 & 277 & 5 & 43 & 956 \\
\hline & 46.36 & 4363 & 61 & 4143 & 37 & 34 & 22 & 23 & 29 & 31 & 3435 & 05 & 35 & 2986 & 2839 & 55 & 81 & 2491 & 3 & 39 & 134 & 1968 & 757 & 1559 & 1399 & 73 & 148 & 1040 & 53 \\
\hline & 45.09 & 4337 & 28 & 4128 & 4015 & 02 & 793 & 3696 & 502 & 10 & 3417 & 293 & 3129 & 2968 & 2826 & 2692 & 569 & 2482 & 2373 & 241 & 2138 & 1964 & 1752 & 1550 & 1391 & 1267 & 1143 & 1035 & 950 \\
\hline & 43.8 & 4319 & 09 & 4102 & 3991 & 3886 & 786 & 3685 & 992 & 3493 & 3410 & 278 & 3109 & 2954 & 2812 & 2674 & 550 & 2463 & 2352 & 2219 & 2117 & 1952 & 1743 & 1546 & 1386 & 258 & 136 & 1030 & 945 \\
\hline & 4 & 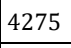 & 4 & 48 & 950 & 45 & 43 & 3642 & 50 & 61 & 3370 & 37 & 8 & 25 & 84 & 52 & 26 & 36 & 25 & 93 & 5 & 1 & 24 & 528 & 69 & 245 & 24 & 018 & DJ1 \\
\hline & 4 & 4232 & 0 & 18 & 3 & 3800 & 6 & 6 & 6 & 4 & 35 & 9 & 5 & 91 & 51 & 0 & 99 & 6 & 2 & 69 & 9 & 6 & 707 & 509 & 1355 & 1233 & 110 & 1003 & 10 \\
\hline & 40 & 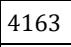 & 2 & 77 & 48 & 3744 & 8 & 6 & 4 & 9 & 3 & 5 & 9 & 1 & 2713 & 9 & 9 & 6 & 6 & 40 & 1 & 8 & 99 & 89 & 8 & 5 & 93 & 0 & 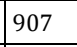 \\
\hline & 3 & 4103 & 3999 & 3895 & 89 & 3679 & 3589 & 5 & 2 & 9 & 5 & 0 & 2 & 7 & 52 & 9 & 2 & 9 & 0 & 3 & 7 & 2 & 3 & 58 & 0 & 39 & 73 & 2 & 888 \\
\hline & 37.47 & 4017 & 3920 & 3819 & 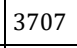 & 3609 & 3515 & 6 & 5 & 3246 & 3159 & 3043 & & 5 & $1 \pm$ & & 4 & 7 & 2 & 55 & 2 & 1 & 3 & 8 & 32 & 66 & 52 & 4 & 07 \\
\hline & & 3920 & 5 & 19 & 1 & 27 & 33 & 3 & 55 & 5 & 91 & 0 & 4 & 34 & 47 & 4 & 2309 & 2001 & | 2128 & 2007 & 1 & 1767 & 107 & 1399 & 1254 & 1141 & 1030 & 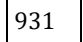 & . \\
\hline
\end{tabular}


Table D.4. Helium concentration in beryllium reflector with LEU core at $12 \mathrm{MW}$ over 8 years operation (appm) - continued

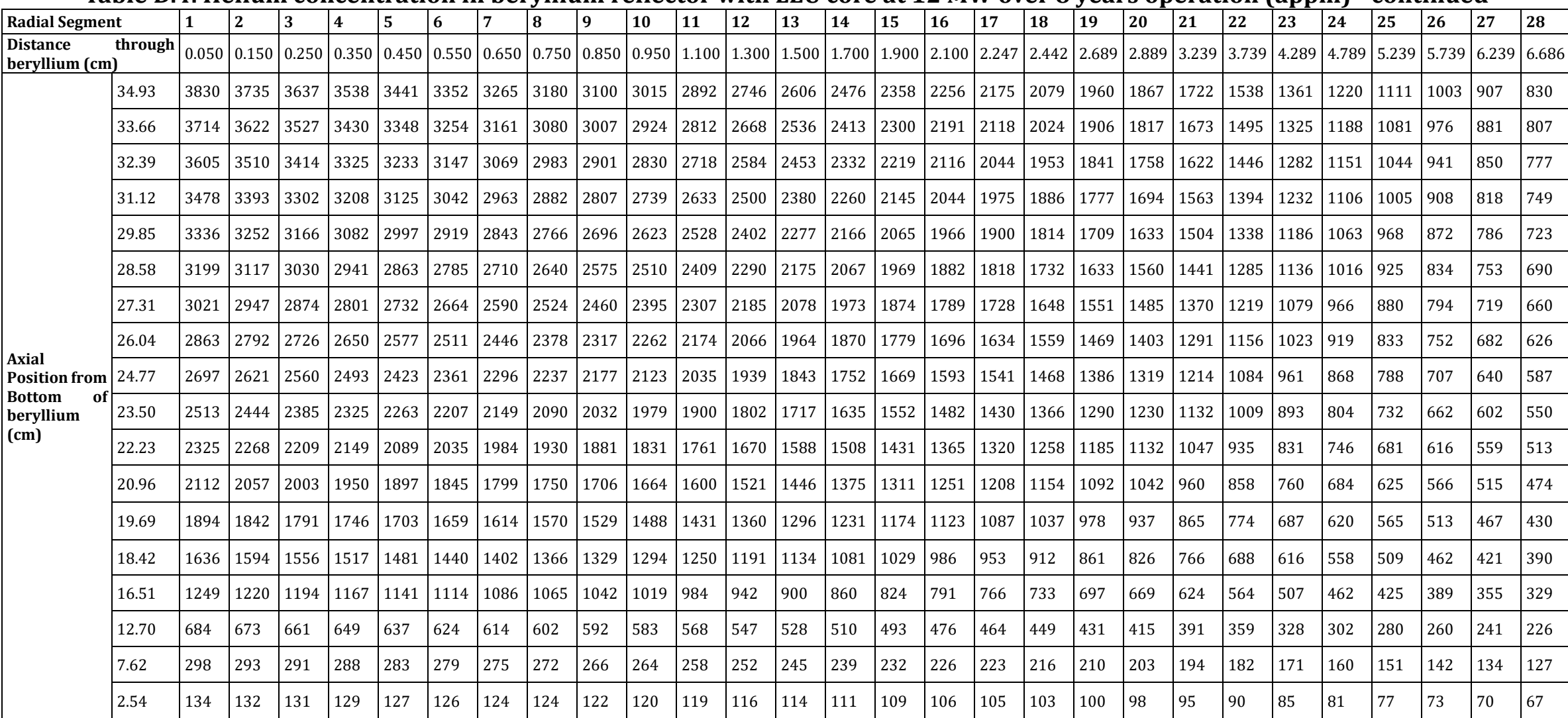


Table D.5. Neutron damage in beryllium reflector with HEU core at $10 \mathrm{MW}$ over 8 years operation (dpa)

\begin{tabular}{|c|c|c|c|c|c|c|c|c|c|c|c|c|c|c|c|c|c|c|c|c|c|c|c|c|c|c|c|c|c|}
\hline \multicolumn{2}{|c|}{\begin{tabular}{|l|} 
Radial Segment \\
\end{tabular}} & \multirow{2}{*}{\begin{tabular}{|l|}
1 \\
0.050 \\
\end{tabular}} & \multirow{2}{*}{\begin{tabular}{|l|}
2 \\
0.150
\end{tabular}} & \multirow{2}{*}{\begin{tabular}{|l|}
3 \\
0.250
\end{tabular}} & \multirow{2}{*}{\begin{tabular}{|l|}
4 \\
0.350
\end{tabular}} & \multirow{2}{*}{\begin{tabular}{|l|}
$\mathbf{5}$ \\
0.450
\end{tabular}} & \multirow{2}{*}{\begin{tabular}{|l|}
6 \\
0.550
\end{tabular}} & \multirow{2}{*}{\begin{tabular}{|l|}
7 \\
0.650
\end{tabular}} & \multirow{2}{*}{\begin{tabular}{|l|}
8 \\
0.750 \\
\end{tabular}} & \multirow{2}{*}{\begin{tabular}{|l|}
$\mathbf{9}$ \\
0.850
\end{tabular}} & \multirow{2}{*}{\begin{tabular}{|l|}
$\mathbf{1 0}$ \\
0.950
\end{tabular}} & \multirow{2}{*}{\begin{tabular}{|l|}
11 \\
1.100 \\
\end{tabular}} & & \multirow{2}{*}{$\begin{array}{l}13 \\
1.500\end{array}$} & & & \multirow{2}{*}{\begin{tabular}{|l|}
16 \\
2.100 \\
\end{tabular}} & \multirow{2}{*}{\begin{tabular}{|l|}
$\mathbf{1 7}$ \\
2.247 \\
\end{tabular}} & & \multirow{2}{*}{\begin{tabular}{|l|}
19 \\
2.689 \\
\end{tabular}} & 20 & 21 & 22 & 23 & 24 & 25 & 26 & 27 & 28 \\
\hline \begin{tabular}{|l|}
$\begin{array}{l}\text { Distance } \\
\text { beryllium }\end{array}$ \\
\end{tabular} & $\begin{array}{l}\text { through } \\
\text { (cm) }\end{array}$ & & & & & & & & & & & & & & & & & & & & 2.889 & 3.239 & 3.739 & 4.289 & 4.789 & 5.239 & 5.739 & 6.239 & 6.686 \\
\hline & 91.44 & 0.45 & 0.46 & 0.46 & 0.46 & 0.46 & 0.46 & 0.45 & 0.45 & 0.45 & 0.45 & 0.45 & 0.45 & 0.44 & 0.44 & 0.43 & 0.42 & 0.42 & 0.41 & 0.40 & 0.40 & 0.39 & 0.37 & 0.35 & 0.34 & 0.32 & 0.31 & 0.29 & 0.27 \\
\hline & 86.36 & 1.23 & 1.23 & 1.23 & 1.23 & 1.23 & 1.22 & 1.22 & 1.21 & 1.21 & 1.20 & 1.19 & 1.17 & 1.16 & 1.13 & 1.11 & 1.08 & 1.07 & 1.05 & 1.02 & 1.00 & 0.96 & 0.91 & 0.86 & 0.81 & 0.77 & 0.72 & 0.67 & 0.62 \\
\hline & 81.28 & 3.01 & 3.00 & 2.98 & 2.96 & 2.94 & 2.92 & 2.89 & 2.86 & 2.83 & 2.80 & 2.76 & 2.70 & 2.64 & 2.56 & 2.48 & 2.41 & 2.36 & 2.30 & 2.22 & 2.16 & 2.06 & 1.91 & 1.76 & 1.63 & 1.53 & 1.42 & 1.29 & 1.17 \\
\hline & 77.47 & 5.18 & 5.14 & 5.10 & 5.04 & 4.98 & 4.92 & 4.86 & 4.80 & 4.73 & 4.67 & 4.57 & 4.45 & 4.32 & 4.19 & 4.07 & 3.95 & 3.87 & 3.76 & 3.61 & 3.50 & 3.31 & 3.05 & 2.78 & 2.54 & 2.34 & 2.12 & 1.91 & 1.71 \\
\hline & 74.93 & 6.74 & 6.69 & 6.62 & 6.54 & 6.46 & 6.38 & 6.29 & 6.21 & 6.12 & 6.03 & 5.89 & 5.72 & 5.55 & 5.38 & 5.21 & 5.06 & 4.95 & 4.80 & 4.61 & 4.46 & 4.20 & 3.86 & 3.50 & 3.19 & 2.92 & 2.64 & 2.37 & 2.12 \\
\hline & 72.39 & 8.12 & 8.07 & .00 & .92 & 7.81 & 7.72 & 7.61 & 7.51 & 7.40 & 7.30 & 7.14 & 6.94 & 6.73 & 6.53 & 6.33 & 6.13 & 6.00 & 5.81 & 5.58 & 5.39 & 5.08 & 4.65 & 4.21 & 3.83 & 3.51 & 3.16 & 2.83 & 2.53 \\
\hline & $\mid 69.85$ & 9.55 & 9.49 & 9.39 & 9.27 & 9.16 & 9.05 & 8.93 & 8.80 & 8.69 & 8.56 & 8.38 & 8.13 & 7.88 & 7.64 & 7.39 & 7.17 & 7.01 & 6.79 & 6.52 & 6.30 & 5.93 & 5.42 & 4.90 & 4.46 & 4.07 & 3.67 & 3.28 & 2.92 \\
\hline & 67.31 & 10.85 & 10.78 & 10.68 & 10.56 & 10.43 & 10.30 & 10.16 & 10.01 & 9.87 & 9.74 & 9.52 & 9.23 & 8.95 & 8.67 & 8.40 & 8.14 & 7.95 & 7.70 & 7.39 & 7.14 & 6.71 & 6.14 & 5.55 & 5.04 & 4.60 & 4.15 & 3.70 & 3.30 \\
\hline & 64.77 & 2.03 & 11.95 & 11.82 & 11.69 & 11.55 & 11.40 & 11.24 & 1.08 & 10.93 & 10.78 & 10.53 & 10.22 & 9.90 & 50 & 30 & 01 & 80 & 53 & 18 & .91 & 7.43 & 6.80 & 14 & 5.57 & 5.09 & 58 & 4.09 & 3.64 \\
\hline & 62.23 & 13.02 & 12.94 & 12.81 & 12.67 & 12.51 & 12.36 & 12.19 & 12.01 & 11.84 & 11.67 & 11.42 & 11.07 & 10.74 & 10.41 & 10.08 & 9.77 & 9.54 & 9.24 & 8.87 & 8.57 & 8.06 & 7.37 & 6.65 & 6.04 & 5.51 & 4.96 & 4.42 & 3.94 \\
\hline & 59.69 & 13.89 & 13.80 & 13.66 & 13.51 & 13.33 & 13.16 & 12.98 & 12.80 & 12.63 & 12.46 & 12.18 & 11.80 & 11.44 & 11.09 & 10.74 & 10.41 & 10.17 & 9.84 & 9.45 & 9.13 & 8.59 & 7.85 & 7.09 & 6.43 & 5.88 & 5.28 & 4.71 & 4.19 \\
\hline & 5 & 4.59 & 47 & 14.33 & 14.17 & .99 & 13.82 & 13.63 & 3.44 & 13.26 & .07 & 12.79 & 12.40 & 12.03 & 1.65 & 11.28 & 10.93 & 0.68 & 10.35 & 9.92 & 9.59 & 9.02 & 8.24 & 44 & 5.76 & 5.17 & 55 & 4.95 & 4.40 \\
\hline & 54.61 & 15.09 & 14.99 & 14.85 & 14.68 & 14.50 & 14.32 & 14.14 & 13.94 & 13.74 & 13.54 & 13.24 & 12.84 & 12.45 & 12.07 & 11.69 & 11.34 & 11.08 & 10.73 & 10.29 & 9.94 & 9.35 & 8.55 & 7.72 & 7.00 & 6.40 & 5.75 & 5.12 & 4.56 \\
\hline & 52.71 & 5.38 & .29 & 15.13 & 14.95 & .78 & 14.59 & 39 & .18 & 13.97 & 13.78 & 13.47 & 13.08 & 12.68 & 2.29 & 11.91 & 11.54 & 1.28 & 10.92 & 10.48 & 10.11 & 9.53 & 8.71 & 7.85 & 7.13 & 6.51 & 5.85 & 5.22 & 4.64 \\
\hline & 51.44 & 15.50 & 15.40 & 15.25 & 15.08 & 14.90 & 14.71 & 14.52 & 14.31 & 14.12 & 13.92 & 13.61 & 13.19 & 12.80 & 12.41 & 12.02 & 11.66 & 11.39 & 11.02 & 10.56 & 10.20 & 9.60 & 8.77 & 7.92 & 7.19 & 6.56 & 5.90 & 5.25 & 4.66 \\
\hline & 5 & 63 & 51 & 36 & 19 & .99 & 14.80 & 60 & 40 & 20 & 01 & .71 & 29 & 12.89 & .48 & .10 & 11.73 & 47 & 11.09 & 10.63 & 28 & 9.67 & 8.83 & 96 & 22 & 6.60 & 93 & .27 & 59 \\
\hline & 48.90 & 15.68 & 15.57 & 15.42 & 15.25 & 15.05 & 14.85 & 14.67 & 14.48 & 14.28 & 14.07 & 13.76 & 13.34 & 12.92 & 12.53 & 12.14 & 11.78 & 11.51 & 11.13 & 10.68 & 10.32 & 9.69 & 8.8 & 7.99 & 7.25 & 6.60 & 5.92 & 5.27 & 4.68 \\
\hline & 47.63 & 15.69 & 15.60 & 15.43 & 15.26 & 15.07 & 14.88 & 14.69 & 14.49 & 14.29 & 14.08 & 13.76 & 13.37 & 12.95 & 12.56 & 12.16 & 11.78 & 11.51 & 11.15 & 10.68 & 10.30 & 9.70 & 8.86 & 7.99 & 7.24 & 6.60 & 5.92 & 5.27 & 4.67 \\
\hline & 46.36 & 15.68 & 15.58 & 15.42 & 15.24 & 15.06 & 14.86 & 14.67 & 14.47 & 14.26 & 14.05 & 13.74 & 13.34 & 12.94 & 12.53 & 12.14 & 11.76 & 11.50 & 11.13 & 10.67 & 10.30 & 9.68 & 8.84 & 97 & 7.22 & 6.57 & 89 & 5.24 & 4.6 \\
\hline & 45.09 & 15.62 & 15.53 & 15.35 & 15.19 & 15.00 & 14.81 & 14.62 & 14.41 & 14.20 & 14.01 & 13.70 & 13.28 & 12.88 & 12.48 & 12.07 & 11.70 & 11.44 & 11.07 & 10.62 & 10.26 & 9.64 & 8.80 & 7.93 & 7.19 & 6.54 & 5.87 & 5.20 & 4.60 \\
\hline & 43.82 & 15.52 & 43 & 15.27 & 15.11 & 14.91 & 14.73 & 14.52 & 14.33 & 14.12 & 13.92 & 13.60 & 13.20 & 12.80 & 12.39 & 11.98 & 11.62 & 11.35 & 11.01 & 10.56 & 10.19 & 9.58 & 8.74 & 37 & 7.13 & 6.49 & 81 & 5.15 & 4. \\
\hline & 42 & 39 & 30 & 14 & 7 & 77 & 59 & 39 & 19 & 00 & 79 & 48 & 08 & 68 & 28 & 89 & 52 & 26 & 91 & 45 & 09 & 48 & 65 & 79 & 06 & .42 & 75 & 10 & 4.51 \\
\hline & 41.28 & 15.22 & 15.11 & 14.95 & 14.80 & 14.62 & 14.43 & 14.24 & 14.04 & 13.84 & 13.63 & 13.34 & 12.93 & 12.54 & 12.14 & 11.76 & 11.39 & 11.13 & 10.78 & 10.33 & 9.97 & 9.37 & 8.55 & 59 & 6.97 & 6.35 & 69 & 5.04 & 43 \\
\hline & 40.01 & 15.01 & 14.92 & 14.77 & 14.60 & 14.41 & 14.23 & 14.04 & 13.85 & 13.65 & 13.46 & 13.17 & 12.76 & 12.37 & 11.99 & 11.60 & 11.24 & 10.98 & 10.63 & 10.18 & 9.82 & 9.24 & 0.15 & 7.58 & 6.87 & 6.26 & 5.60 & 4.96 & 10 \\
\hline & 38.74 & 14.77 & 14.66 & 14.51 & 14.35 & 14.18 & 14.00 & 13.82 & 13.63 & 13.44 & 13.23 & 12.93 & 12.54 & 12.14 & 11.77 & 11.38 & 11.04 & 10.79 & 10.44 & 10.01 & 9.66 & 9.09 & 8.29 & 7.46 & 6.75 & 6.15 & 5.50 & 4.87 & 4.29 \\
\hline & 3 & 4.50 & 40 & 26 & 09 & 13.91 & .73 & .54 & 35 & 16 & 96 & .69 & 31 & 93 & .57 & 19 & 83 & .59 & 25 & 32 & 8 & 91 & 12 & 31 & 61 & .02 & 38 & .76 & 4.20 \\
\hline & 36.20 & 14.16 & 14.07 & 13.92 & 13.78 & 13.60 & 13.43 & 13.24 & 13.06 & 12.88 & 12.69 & 12.40 & 12.02 & 11.65 & 11.30 & 10.94 & 10.60 & 10.36 & 10.02 & 9.60 & 9.28 & 8.72 & 7.95 & 7.15 & 6.46 & 5.88 & 5.25 & 4.65 & 4.09 \\
\hline
\end{tabular}


Table D.6. Neutron damage in beryllium reflector with HEU core at $10 \mathrm{MW}$ over 8 years operation (dpa) - continued

\begin{tabular}{|c|c|c|c|c|c|c|c|c|c|c|c|c|c|c|c|c|c|c|c|c|c|c|c|c|c|c|c|c|c|}
\hline \multirow{2}{*}{\multicolumn{2}{|c|}{\begin{tabular}{|l|} 
Radial Segment \\
$\begin{array}{l}\text { Distance through } \\
\text { beryllium (cm) }\end{array}$ \\
\end{tabular}}} & \multirow{2}{*}{\begin{tabular}{|l|}
$\mathbf{1}$ \\
0.050 \\
\end{tabular}} & \multirow{2}{*}{$\begin{array}{ll}2 \\
0.150\end{array}$} & \multirow{2}{*}{\begin{tabular}{|l|}
3 \\
0.250 \\
\end{tabular}} & \multirow{2}{*}{$\begin{array}{ll}4 \\
0.350 \\
\end{array}$} & \multirow{2}{*}{\begin{tabular}{|l|}
$\mathbf{5}$ \\
0.450 \\
\end{tabular}} & \multirow{2}{*}{\begin{tabular}{|l|}
6 \\
0.550 \\
\end{tabular}} & \multirow{2}{*}{\begin{tabular}{|l|}
7 \\
0.650 \\
\end{tabular}} & \multirow{2}{*}{\begin{tabular}{|l|}
8 \\
0.750 \\
\end{tabular}} & \multirow{2}{*}{\begin{tabular}{|l|}
$\mathbf{9}$ \\
0.850 \\
\end{tabular}} & & \multirow{2}{*}{\begin{tabular}{|l|}
11 \\
1.100 \\
\end{tabular}} & & \multirow{2}{*}{\begin{tabular}{|l|l|}
13 \\
1.500 \\
\end{tabular}} & & \multirow{2}{*}{\begin{tabular}{|l|l|}
15 \\
1.900 \\
\end{tabular}} & & & & & & \multirow{2}{*}{\begin{tabular}{|l|}
21 \\
3.239 \\
\end{tabular}} & \multirow{2}{*}{\begin{tabular}{|l|}
22 \\
3.739 \\
\end{tabular}} & \multirow{2}{*}{\begin{tabular}{|l|}
23 \\
4.289 \\
\end{tabular}} & & 25 & 26 & 27 & 28 \\
\hline & & & & & & & & & & & & & & & & & & & & & & & & & & 5.239 & 5.739 & 239 & 6.686 \\
\hline & 34.93 & 13.79 & 13.70 & 13.57 & 13.42 & 13.25 & 13.07 & 12.90 & 12.73 & 12.54 & 12.36 & 12.08 & 11.73 & 11.37 & 11.01 & 10.66 & 10.32 & 10.09 & 9.76 & 9.36 & 9.04 & 8.49 & 7.74 & 6.96 & 6.28 & 5.72 & 5.11 & 4.52 & 3.98 \\
\hline & 33.66 & 13.42 & 13.32 & 13.19 & 13.05 & 12.88 & 12.72 & 12.54 & 12.37 & 12.18 & 12.01 & 11.76 & 11.40 & 11.05 & 10.70 & 10.37 & 10.04 & 9.81 & 9.50 & 9.09 & 8.78 & 8.24 & 7.51 & 6.76 & 6.11 & 5.56 & 4.96 & 4.38 & 3.85 \\
\hline & 32.39 & 13.00 & .91 & 12.79 & 12.62 & 12.46 & 12.31 & 12.14 & 11.97 & 11.80 & 11.64 & 11.37 & 11.03 & 10.68 & 10.36 & 10.02 & 9.71 & 9.48 & 9.18 & 8.79 & 8.49 & 7.97 & 7.27 & 6.54 & 5.91 & 5.37 & 4.80 & 4.24 & 3.73 \\
\hline & 31.12 & 12.53 & 12.45 & 12.33 & 12.20 & 12.04 & 11.87 & 11.71 & 11.56 & 11.40 & 11.22 & 10.97 & 10.64 & 10.31 & 9.99 & 9.66 & 9.36 & 9.14 & 8.86 & 8.48 & 8.17 & 7.69 & 7.01 & 6.30 & 5.70 & 5.18 & 4.62 & 4.09 & 3.60 \\
\hline & 29.85 & 12.02 & 11.94 & 11.82 & 11.69 & 11.55 & 11.40 & 11.24 & 11.07 & 10.92 & 10.77 & 10.54 & 10.21 & 9.91 & 9.59 & 9.28 & 8.99 & 8.78 & 8.51 & 8.15 & 7.87 & 7.39 & 6.74 & 6.05 & 5.47 & 4.98 & 4.44 & 3.92 & 3.46 \\
\hline & 28.58 & 11.52 & 11.45 & 11.32 & 11.19 & 11.07 & 10.92 & 10.78 & 10.62 & 10.48 & 10.31 & 10.08 & 9.77 & 9.47 & 9.17 & 8.87 & 8.60 & 8.40 & 8.13 & 7.79 & 7.53 & 7.06 & 6.44 & 5.79 & 5.23 & 4.76 & 4.25 & 3.76 & 3.31 \\
\hline & 27.31 & 0.96 & 0.88 & 10.77 & 10.65 & 10.51 & 10.38 & 0.25 & 10.11 & 9.95 & 9.80 & 9.58 & 30 & 9.01 & 72 & 8.44 & 8.17 & 7.99 & 7.73 & 7.41 & 7.16 & 6.72 & 6.12 & 5.51 & 4.99 & 4.53 & 4.05 & 3.58 & 3.15 \\
\hline & 26.04 & 10.36 & 10.30 & 10.19 & 10.07 & 9.94 & 9.80 & 9.67 & 9.54 & 9.41 & 9.27 & 9.05 & 8.78 & 8.51 & 8.24 & 7.98 & 7.73 & 7.56 & 7.31 & 7.00 & 6.76 & 6.35 & 5.80 & 5.21 & 4.71 & 4.28 & 3.83 & 3.39 & 2.99 \\
\hline rom & 24.77 & 9.74 & 9.66 & 9.56 & 9.46 & 9.35 & 9.22 & 9.09 & 8.96 & 8.83 & 8.69 & 8.51 & 8.26 & 8.00 & 7.75 & 7.49 & 7.25 & 7.08 & 6.85 & 6.57 & 6.34 & 5.96 & 5.44 & 4.89 & 4.42 & 4.03 & 3.60 & 3.19 & 2.82 \\
\hline & 3.50 & 06 & 99 & 90 & 79 & 68 & 8.57 & 46 & 33 & 21 & 10 & \begin{tabular}{|l}
7.92 \\
\end{tabular} & 7 & 43 & 20 & 96 & 74 & 59 & 38 & 11 & 5.90 & .55 & 06 & 4.56 & 4.13 & 3.76 & 3.37 & 99 & 2.64 \\
\hline & 22.23 & 8.35 & 8.29 & 8.19 & 8.09 & 8.00 & 7.89 & 7.78 & 7.67 & 7.55 & 7.44 & 7.28 & 7.06 & 6.84 & 6.62 & 6.41 & 6.21 & 6.07 & 5.88 & 5.63 & 5.44 & 5.11 & 4.66 & 4.20 & 3.81 & 3.48 & 3.12 & 2.77 & 2.46 \\
\hline & 20.96 & 7.56 & .51 & 7.43 & .34 & 25 & .15 & 7.06 & 95 & 6.85 & .76 & 6.60 & 6.40 & 6.21 & 6.02 & 5.82 & 5.64 & 5.51 & 5.34 & 5.12 & 4.94 & 4.65 & 4.25 & 3.84 & 3.48 & 3.17 & 2.85 & 2.54 & 2.26 \\
\hline & 19.69 & 6.71 & 6.67 & 6.61 & 6.53 & 45 & 6.37 & 6.29 & 6.20 & 6.11 & .02 & 5.89 & .71 & 5.53 & 5.36 & 5.20 & 5.04 & 4.93 & 4.77 & 4.57 & 4.43 & 4.17 & 3.82 & 3.45 & 3.14 & 2.87 & 2.58 & 2.30 & 2.05 \\
\hline & 18.4 & 5 & 5.78 & 5 & 5.66 & 9 & 52 & 5.46 & . & 5 & 5.23 & 5.12 & 8 & 4.83 & 69 & 4. & 42 & 4.33 & 4.19 & 4.03 & 3.90 & 3.68 & 3.38 & 3.05 & 2.79 & 2.55 & 2.30 & 2.06 & 1.84 \\
\hline & 16.51 & 4.42 & 4.40 & 4.37 & 4.34 & 4.29 & 4.25 & 4.21 & 1.0 & 4.12 & 4.07 & 4.00 & 3.90 & 3 & 70 & 3.60 & 51 & 44 & 35 & 3.22 & 3.13 & 2.96 & 2.73 & 2.49 & 2.28 & 2.10 & 1.90 & 70 & 1.52 \\
\hline & 12.70 & 2.32 & 2.33 & 2.32 & 2.32 & 2.31 & 2.30 & 2.29 & 2.27 & 2.26 & 2.24 & 2.21 & 18 & 2.14 & 2.10 & 2.06 & 2.02 & 1.99 & 1.95 & 1.89 & 1.85 & 1.77 & 1.66 & 1.53 & 1.42 & 1.32 & 1.21 & 1.09 & 0.99 \\
\hline & 7.62 & 0.89 & 0.90 & 0.91 & 0.91 & 0.91 & 0.91 & 0.91 & 0.91 & 0.90 & 0.90 & 0.90 & 89 & 0.88 & 87 & 0.86 & 0.85 & 0.84 & 0.83 & 0.81 & 0.80 & 0.78 & 0.75 & 0.70 & 0.66 & 0.62 & 0.58 & 0.54 & 0.50 \\
\hline & 34 & 0.33 & 0.33 & 0.33 & 0.33 & 0.34 & 0.34 & 0.34 & 0.34 & 0.34 & 0.34 & 0.34 & 0.04 & 0.33 & 0.33 & 0.03 & 0.33 & 0.32 & 0.32 & 0.02 & 0.0 & 1. & 0.30 & 0.28 & 0.2 & 0.26 & 0.24 & 0.23 & 0.22 \\
\hline
\end{tabular}


Table D.7. Neutron damage in beryllium reflector with LEU core at $12 \mathrm{MW}$ over 8 years operation (dpa)

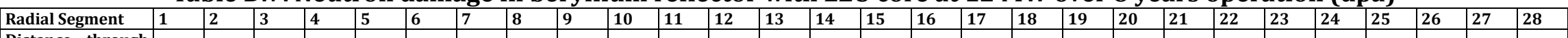

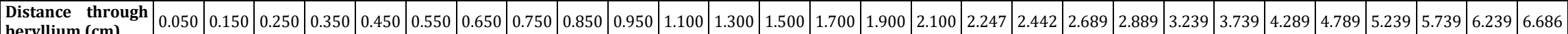

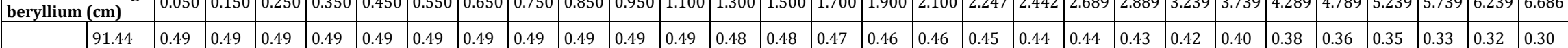

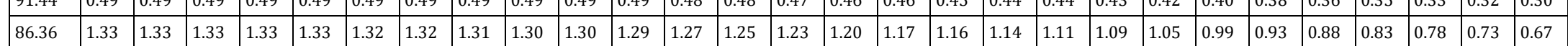
\begin{tabular}{|l|l|l|l|l|l|l|l|l|l|l|l|l|l|l|l|l|l|l|l|l|l|l|l|l|l|l|l|l|l|l|l|l|l|l|l|l|l|l|l|l|l|}
\hline 81.28 & 3.29 & 3.28 & 3.26 & 3.24 & 3.22 & 3.19 & 3.16 & 3.12 & 3.09 & 3.06 & 3.01 & 2.95 & 2.88 & 2.80 & 2.71 & 2.63 & 2.58 & 2.51 & 2.43 & 2.36 & 2.24 & 2.09 & 1.92 & 1.78 & 1.67 & 1.54 & 1.40 & 1.27 \\
\hline
\end{tabular} \begin{tabular}{|l|l|l|l|l|l|l|l|l|l|l|l|l|l|l|l|l|l|l|l|l|l|l|l|l|l|l|l|l|l|l|l|l|}
\hline 77.47 & 5.72 & 5.69 & 5.63 & 5.56 & 5.50 & 5.42 & 5.36 & 5.29 & 5.22 & 5.14 & 5.04 & 4.90 & 4.76 & 4.62 & 4.48 & 4.36 & 4.26 & 4.13 & 3.97 & 3.85 & 3.64 & 3.35 & 3.04 & 2.78 & 2.56 & 2.32 & 2.09 & 1.88 \\
\hline
\end{tabular} \begin{tabular}{|l|l|l|l|l|l|l|l|l|l|l|l|l|l|l|l|l|l|l|l|l|l|l|l|l|l|l|l|l|l|l|}
\hline 74.93 & 7.43 & 7.38 & 7.30 & 7.21 & 7.13 & 7.03 & 6.94 & 6.84 & 6.74 & 6.65 & 6.50 & 6.31 & 6.12 & 5.93 & 5.75 & 5.58 & 5.46 & 5.29 & 5.08 & 4.91 & 4.62 & 4.24 & 3.84 & 3.50 & 3.21 & 2.90 & 2.60 & 2.33 \\
\hline
\end{tabular} \begin{tabular}{|l|l|l|l|l|l|l|l|l|l|l|l|l|l|l|l|l|l|l|l|l|l|l|l|l|l|l|l|l|l|l|l|l|l|l|l|}
\hline 72.39 & 9.02 & 8.96 & 8.88 & 8.77 & 8.67 & 8.56 & 8.44 & 8.32 & 8.20 & 8.08 & 7.91 & 7.68 & 7.45 & 7.22 & 6.98 & 6.77 & 6.63 & 6.42 & 6.17 & 5.96 & 5.61 & 5.13 & 4.64 & 4.22 & 3.86 & 3.48 & 3.11 & 2.78 \\
\hline
\end{tabular}

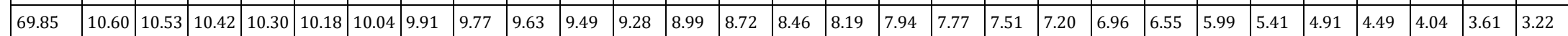
\begin{tabular}{|l|l|l|l|l|l|l|l|l|l|l|l|l|l|l|l|l|l|l|l|l|l|l|l|l|l|l|l|l|l|}
\hline 67.31 & 12.04 & 11.96 & 11.84 & 11.71 & 11.56 & 11.40 & 11.25 & 11.09 & 10.93 & 10.77 & 10.53 & 10.21 & 9.89 & 9.59 & 9.28 & 9.00 & 8.80 & 8.52 & 8.17 & 7.89 & 7.43 & 6.78 & 6.12 & 5.56 & 5.08 & 4.56 & 4.07 & 3.62 \\
\hline
\end{tabular} \begin{tabular}{|l|l|l|l|l|l|l|l|l|l|l|l|l|l|l|l|l|l|l|l|l|l|l|l|l|l|l|l|l|l|l|l|l|l|l|l|}
\hline 64.77 & 13.32 & 13.23 & 13.08 & 12.95 & 12.78 & 12.61 & 12.45 & 12.27 & 12.09 & 11.92 & 11.65 & 11.30 & 10.96 & 10.61 & 10.27 & 9.94 & 9.72 & 9.41 & 9.02 & 8.71 & 8.20 & 7.50 & 6.76 & 6.14 & 5.60 & 5.04 & 4.49 & 4.00 \\
\hline
\end{tabular} \begin{tabular}{|l|l|l|l|l|l|l|l|l|l|l|l|l|l|l|l|l|l|l|l|l|l|l|l|l|l|l|l|l|l|l|l|l|}
\hline 62.23 & 14.42 & 14.32 & 14.17 & 14.01 & 13.83 & 13.67 & 13.48 & 13.29 & 13.10 & 12.90 & 12.61 & 12.23 & 11.86 & 11.48 & 11.11 & 10.76 & 10.52 & 10.19 & 9.78 & 9.45 & 8.88 & 8.11 & 7.32 & 6.64 & 6.07 & 5.46 & 4.86 & 4.33 \\
\hline
\end{tabular} \begin{tabular}{|l|l|l|l|l|l|l|l|l|l|l|l|l|l|l|l|l|l|l|l|l|l|l|l|l|l|l|l|l|l|l|l|l|l|l|l|l|l|l|l|}
\hline 59.69 & 15.33 & 15.23 & 15.07 & 14.90 & 14.72 & 14.54 & 14.34 & 14.14 & 13.94 & 13.74 & 13.43 & 13.02 & 12.62 & 12.23 & 11.85 & 11.47 & 11.20 & 10.85 & 10.40 & 10.05 & 9.45 & 8.63 & 7.78 & 7.07 & 6.45 & 5.80 & 5.17 & 4.60 \\
\hline
\end{tabular}

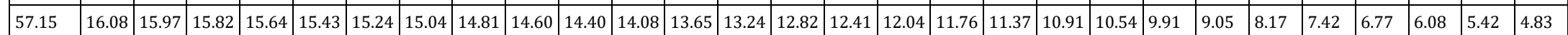
Axial

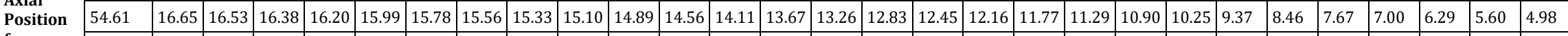

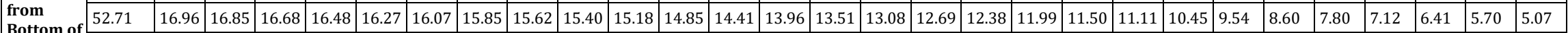

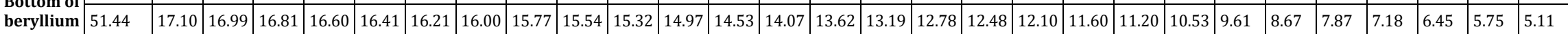

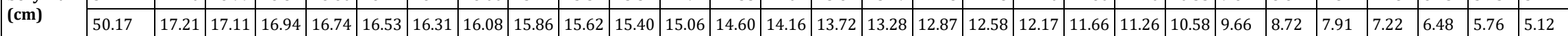

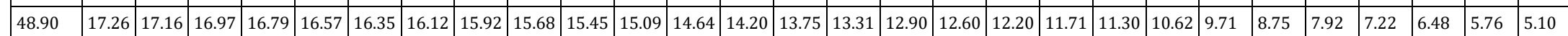

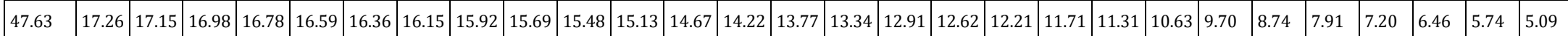

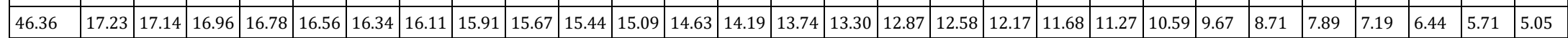

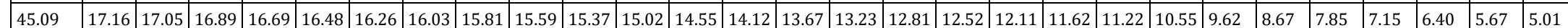
\begin{tabular}{|l|l|l|l|l|l|l|l|l|l|l|l|l|l|l|l|l|l|l|l|l|l|l|l|l|l|l|l|l|l|l|l|l|l|l|}
\hline 43.82 & 17.04 & 16.93 & 16.77 & 16.57 & 16.36 & 16.15 & 15.93 & 15.72 & 15.48 & 15.28 & 14.93 & 14.48 & 14.04 & 13.59 & 13.14 & 12.74 & 12.43 & 12.04 & 11.54 & 11.14 & 10.48 & 9.55 & 8.60 & 7.79 & 7.09 & 6.35 & 5.62 & 4.96 \\
\hline
\end{tabular} \begin{tabular}{|l|l|l|l|l|l|l|l|l|l|l|l|l|l|l|l|l|l|l|l|l|l|l|l|l|l|l|l|l|l|l|l|}
\hline 42.55 & 16.89 & 16.78 & 16.60 & 16.42 & 16.21 & 16.01 & 15.79 & 15.57 & 15.34 & 15.12 & 14.78 & 14.34 & 13.90 & 13.47 & 13.03 & 12.63 & 12.33 & 11.94 & 11.44 & 11.05 & 10.37 & 9.47 & 8.52 & 7.71 & 7.02 & 6.27 & 5.55 & 4.90 \\
\hline
\end{tabular} \begin{tabular}{|l|l|l|l|l|l|l|l|l|l|l|l|l|l|l|l|l|l|l|l|l|l|l|l|l|l|l|l|l|l|l|l|l|l|l|l|}
\hline 41.28 & 16.68 & 16.56 & 16.40 & 16.23 & 16.02 & 15.81 & 15.59 & 15.37 & 15.15 & 14.93 & 14.61 & 14.16 & 13.73 & 13.30 & 12.89 & 12.48 & 12.18 & 11.79 & 11.29 & 10.91 & 10.25 & 9.35 & 8.41 & 7.61 & 6.93 & 6.19 & 5.48 & 4.84 \\
\hline
\end{tabular}

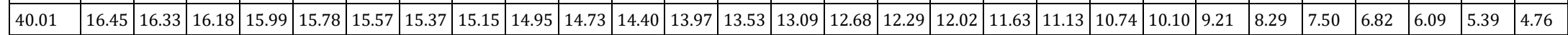
\begin{tabular}{|l|l|l|l|l|l|l|l|l|l|l|l|l|l|l|l|l|l|l|l|l|l|l|l|l|l|l|l|l|l|l|l|l|l|l|}
\hline 38.74 & 16.20 & 16.08 & 15.94 & 15.74 & 15.55 & 15.34 & 15.13 & 14.91 & 14.68 & 14.47 & 14.15 & 13.73 & 13.30 & 12.89 & 12.48 & 12.08 & 11.80 & 11.42 & 10.95 & 10.57 & 9.93 & 9.05 & 8.14 & 7.35 & 6.69 & 5.98 & 5.30 & 4.66 \\
\hline
\end{tabular} \begin{tabular}{|l|l|l|l|l|l|l|l|l|l|l|l|l|l|l|l|l|l|l|l|l|l|l|l|l|l|l|l|l|l|l|l|l|l|l|}
\hline 37.47 & 15.86 & 15.76 & 15.61 & 15.40 & 15.22 & 15.03 & 14.84 & 14.63 & 14.41 & 14.19 & 13.87 & 13.47 & 13.05 & 12.64 & 12.23 & 11.85 & 11.57 & 11.19 & 10.73 & 10.36 & 9.72 & 8.86 & 7.97 & 7.20 & 6.54 & 5.85 & 5.18 & 4.56 \\
\hline
\end{tabular} \begin{tabular}{|l|l|l|l|l|l|l|l|l|l|l|l|l|l|l|l|l|l|l|l|l|l|l|l|l|l|l|l|l|l|}
\hline 36.20 & 15.50 & 15.39 & 15.23 & 15.05 & 14.88 & 14.69 & 14.49 & 14.28 & 14.08 & 13.87 & 13.56 & 13.15 & 12.75 & 12.34 & 11.94 & 11.57 & 11.31 & 10.94 & 10.47 & 10.11 & 9.49 & 8.65 & 7.78 & 7.03 & 6.38 & 5.70 & 5.05 & 4.45 \\
\hline
\end{tabular} 
Table D.8. Neutron damage in beryllium reflector with LEU core at $12 \mathrm{MW}$ over 8 years operation (dpa) - continued

\begin{tabular}{|c|c|c|c|c|c|c|c|c|c|c|c|c|c|c|c|c|c|c|c|c|c|c|c|c|c|c|c|c|c|}
\hline \multirow{2}{*}{\multicolumn{2}{|c|}{\begin{tabular}{|l|} 
Radial Segment \\
$\begin{array}{l}\text { Distance through } \\
\text { beryllium (cm) }\end{array}$ \\
\end{tabular}}} & \multirow{2}{*}{\begin{tabular}{|l|}
1 \\
0.050 \\
\end{tabular}} & \multirow{2}{*}{\begin{tabular}{|l|}
2 \\
0.150 \\
\end{tabular}} & \multirow{2}{*}{\begin{tabular}{|l|}
$\mathbf{3}$ \\
0.250 \\
\end{tabular}} & \multirow{2}{*}{$\begin{array}{ll}4 \\
0.350 \\
\end{array}$} & \multirow{2}{*}{\begin{tabular}{|l|}
$\mathbf{5}$ \\
0.450 \\
\end{tabular}} & \multirow{2}{*}{\begin{tabular}{ll|}
6 \\
0.550 \\
\end{tabular}} & \multirow{2}{*}{\begin{tabular}{|l|}
7 \\
0.650 \\
\end{tabular}} & \multirow{2}{*}{\begin{tabular}{|l|}
8 \\
0.750 \\
\end{tabular}} & \multirow{2}{*}{\begin{tabular}{|l|}
9 \\
0.850 \\
\end{tabular}} & \multirow{2}{*}{\begin{tabular}{|l|}
$\mathbf{1 0}$ \\
0.950 \\
\end{tabular}} & & & & & \multirow{2}{*}{\begin{tabular}{|l|}
15 \\
1.900 \\
\end{tabular}} & & & & & \multirow{2}{*}{\begin{tabular}{|l|}
$\mathbf{2 0}$ \\
2.889 \\
\end{tabular}} & \multirow{2}{*}{\begin{tabular}{|l|}
$\mathbf{2 1}$ \\
3.239 \\
\end{tabular}} & \multirow{2}{*}{\begin{tabular}{|l|}
22 \\
3.739 \\
\end{tabular}} & \multirow{2}{*}{23} & & 25 & 26 & 27 & 28 \\
\hline & & & & & & & & & & & & & & & & & & & & & & & & & & 5.239 & 5.739 & 6.239 & 6.686 \\
\hline & 34.93 & 15.12 & 15.01 & 14.86 & 14.69 & 14.50 & 14.31 & 14.11 & 13.91 & 13.74 & 13.53 & 13.22 & 12.81 & 12.41 & 12.03 & 11.63 & 11.27 & 11.01 & 10.65 & 10.21 & 9.86 & 9.24 & 8.43 & 7.58 & 6.85 & 6.21 & 5.55 & 4.91 & 4.32 \\
\hline & 33.66 & 14.66 & 14.57 & 14.42 & 14.24 & 14.07 & 13.89 & 13.69 & 13.49 & 13.30 & 13.10 & 12.81 & 12.42 & 12.04 & 11.67 & 11.31 & 10.95 & 10.69 & 10.35 & 9.91 & 9.56 & 8.99 & 8.19 & 7.36 & 6.65 & 6.04 & 5.39 & 4.76 & 4.19 \\
\hline & 32.39 & 14.18 & 14.08 & 13.96 & 13.79 & 13.62 & 13.45 & 13.27 & 13.08 & 12.89 & 12.71 & 12.41 & 12.03 & 11.66 & 11.29 & 10.94 & 10.58 & 10.34 & 10.00 & 9.58 & 9.25 & 8.69 & 7.92 & 7.12 & 6.43 & 5.84 & 5.22 & 4.61 & 4.04 \\
\hline & 31.12 & 13.67 & 13.58 & 13.45 & 13.29 & 13.13 & 12.95 & 12.78 & 12.59 & 12.41 & 12.24 & 11.95 & 11.58 & 11.22 & 10.88 & 10.53 & 10.20 & 9.97 & 9.65 & 9.24 & 8.91 & 8.37 & 7.63 & 6.85 & 6.20 & 5.63 & 5.02 & 4.43 & 3.90 \\
\hline & 29.85 & 13.15 & 13.06 & 12.92 & 12.77 & 12.61 & 12.44 & 12.27 & 12.10 & 11.92 & 11.75 & 11.49 & 11.14 & 10.79 & 10.43 & 10.09 & 9.78 & 9.54 & 9.24 & 8.85 & 8.56 & 8.03 & 7.32 & 6.58 & 5.94 & 5.41 & 4.82 & 4.25 & 3.74 \\
\hline & 28.58 & 12.57 & 12.47 & 12.34 & 12.20 & 12.05 & 11.90 & 11.74 & 11.56 & 11.40 & 11.24 & 10.98 & 10.66 & 10.31 & 9.98 & 9.67 & 9.36 & 9.14 & 8.84 & 8.47 & 8.17 & 7.67 & 6.99 & 6.28 & 5.68 & 5.16 & 4.61 & 4.08 & 3.59 \\
\hline & 27.31 & 11.91 & 11.84 & 11.72 & 11.60 & 11.45 & 11.30 & 11.15 & 10.98 & 10.83 & 10.68 & 10.44 & 10.11 & 9.81 & 9.49 & 9.19 & 8.90 & 8.70 & 8.41 & 8.05 & 7.77 & 7.29 & 6.64 & 5.97 & 5.40 & 4.90 & 4.38 & 3.88 & 3.41 \\
\hline & 26.04 & 11.29 & 11.21 & 11.11 & 10.97 & 10.84 & 10.68 & 10.54 & 10.39 & 10.23 & 10.09 & 9.85 & 9.55 & 9.24 & 8.95 & 8.66 & 8.40 & 8.19 & 7.93 & 7.60 & 7.34 & 6.89 & 6.28 & 5.65 & 5.11 & 4.65 & 4.15 & 3.66 & 3.23 \\
\hline from & 24.77 & 10.60 & 10.52 & 10.42 & 10.30 & 10.16 & 10.02 & 9.89 & 9.75 & 9.60 & 9.46 & 9.25 & 8.96 & 8.68 & 8.40 & 8.13 & 7.88 & 7.70 & 7.45 & 7.13 & 6.88 & 6.46 & 5.89 & 5.30 & 4.79 & 4.37 & 3.90 & 3.45 & 3.05 \\
\hline & 23.50 & 9.86 & 9.78 & 9.69 & 9.58 & 9.45 & 9.33 & 9.20 & 9.06 & 8.94 & 8.81 & 8.60 & 8.34 & 8.07 & 7.81 & 7.55 & 7.31 & 7.14 & 6.91 & 6.63 & 6.39 & 6.00 & 5.48 & 4.93 & 4.46 & 4.06 & 3.64 & 3.23 & 2.86 \\
\hline & 22.23 & 9.07 & 9.02 & 8.92 & 8.80 & 8.68 & 8.57 & 8.45 & 8.33 & 8.22 & 8.10 & 7.91 & 7.65 & 7.40 & 7.18 & 6.95 & 6.73 & 6.58 & 6.36 & 6.10 & 5.89 & 5.53 & 5.05 & 4.55 & 4.12 & 3.75 & 3.36 & 2.99 & 2.65 \\
\hline & 20.96 & 8.23 & 8.16 & 8.07 & 7.98 & 7.88 & 7.78 & 7.67 & 7.55 & 7.44 & 7.33 & 7.16 & 6.95 & 6.73 & 6.52 & 6.31 & 6.12 & 5.98 & 5.78 & 5.55 & 5.35 & 5.03 & 4.60 & 4.15 & 3.76 & 3.43 & 3.08 & 2.74 & 2.43 \\
\hline & 19.69 & 7.31 & 7.25 & 7.17 & 7.09 & 7.01 & 6.92 & 6.81 & 6.71 & 6.61 & 6.52 & 6.38 & 6.19 & 6.00 & 5.82 & 5.63 & 5.46 & 5.33 & 5.17 & 4.95 & 4.79 & 4.51 & 4.12 & 3.73 & 3.38 & 3.09 & 2.77 & 2.48 & 2.21 \\
\hline & 18.42 & 6.28 & 6.25 & 19 & 6.12 & 6.06 & 5.98 & .90 & 5.82 & 74 & 5.66 & 5.54 & 5.38 & 5.23 & 5.07 & 4.91 & 4.78 & 4.67 & 4.53 & 4.35 & 4.20 & 3.96 & 3.64 & 3.30 & 3.00 & 2.75 & 2.48 & 2.22 & 1.98 \\
\hline & 16.51 & 4.75 & 4.74 & 4.70 & 4.66 & 4.63 & 4.58 & 4.53 & 4.49 & 4.44 & 4.38 & 4.30 & 4.20 & 4.09 & 3.98 & 3.87 & 3.77 & 3.70 & 3.59 & 3.46 & 3.36 & 3.18 & 2.94 & 2.67 & 2.45 & 2.25 & 2.03 & 1.82 & 1.63 \\
\hline & 12.70 & 2.47 & 2.48 & 2.47 & 2.47 & 2.46 & 2.45 & 2.44 & 2.42 & 2.41 & 2.39 & 2.36 & 2.33 & 2.29 & 2.25 & 2.20 & 2.16 & 2.13 & 2.08 & 2.02 & 1.98 & 1.89 & 1.77 & 1.64 & 1.52 & 1.41 & 1.29 & 1.17 & 1.06 \\
\hline & 7.62 & 0.95 & 0.95 & 0.96 & 0.96 & 0.96 & 0.96 & 0.96 & 0.96 & 0.96 & 0.96 & 0.95 & 0.95 & 0.94 & 0.93 & 0.92 & 0.91 & 0.90 & 0.89 & 0.87 & 0.86 & 0.83 & 0.79 & 0.75 & 0.71 & 0.67 & 0.62 & 0.57 & 0.53 \\
\hline & 2.54 & 0.35 & 0.35 & 0.35 & 0.36 & 0.36 & 0.36 & 0.36 & 0.36 & 0.36 & 0.36 & 0.36 & 0.36 & 0.35 & 0.35 & 0.35 & 0.35 & 0.34 & 0.34 & 0.34 & 0.33 & 0.33 & 0.31 & 0.30 & 0.29 & 0.28 & 0.26 & 0.24 & 0.23 \\
\hline
\end{tabular}


Table D.9. Neutron and photon heating in beryllium reflector with HEU core at $10 \mathrm{MW}$ over 8 years operation (W/ $\mathrm{cm}^{3}$ )

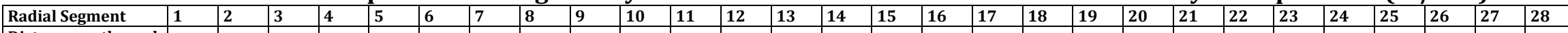

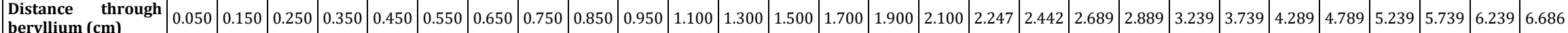

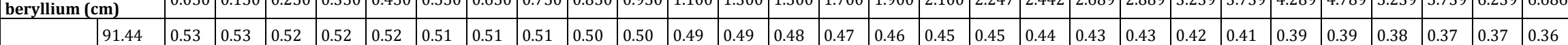

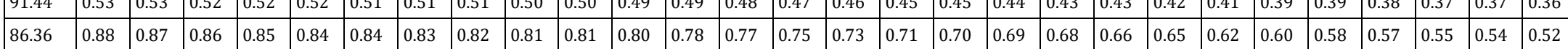
\begin{tabular}{|l|l|l|l|l|l|l|l|l|l|l|l|l|l|l|l|l|l|l|l|l|l|l|l|l|l|l|l|l|l|l|l|l|l|l|l|l|}
\hline 81.28 & 1.47 & 1.45 & 1.43 & 1.41 & 1.40 & 1.38 & 1.36 & 1.35 & 1.33 & 1.32 & 1.29 & 1.26 & 1.24 & 1.20 & 1.17 & 1.14 & 1.12 & 1.09 & 1.06 & 1.04 & 1.00 & 0.95 & 0.91 & 0.87 & 0.84 & 0.82 & 0.79 & 0.76 \\
\hline
\end{tabular} \begin{tabular}{|l|l|l|l|l|l|l|l|l|l|l|l|l|l|l|l|l|l|l|l|l|l|l|l|l|l|l|l|l|l|l|l|l|l|l|l|l|l|l|}
\hline 77.47 & 2.15 & 2.11 & 2.08 & 2.05 & 2.02 & 1.99 & 1.96 & 1.93 & 1.90 & 1.88 & 1.84 & 1.79 & 1.74 & 1.70 & 1.65 & 1.61 & 1.58 & 1.55 & 1.50 & 1.46 & 1.41 & 1.33 & 1.25 & 1.18 & 1.13 & 1.07 & 1.02 & 0.98 \\
\hline
\end{tabular} \begin{tabular}{|l|l|l|l|l|l|l|l|l|l|l|l|l|l|l|l|l|l|l|l|l|l|l|l|l|l|l|l|l|l|l|l|l|}
\hline 74.93 & 2.67 & 2.62 & 2.58 & 2.54 & 2.50 & 2.46 & 2.42 & 2.38 & 2.35 & 2.31 & 2.26 & 2.20 & 2.13 & 2.07 & 2.02 & 1.96 & 1.92 & 1.87 & 1.81 & 1.77 & 1.69 & 1.59 & 1.49 & 1.40 & 1.33 & 1.26 & 1.20 & 1.15 \\
\hline
\end{tabular} \begin{tabular}{|l|l|l|l|l|l|l|l|l|l|l|l|l|l|l|l|l|l|l|l|l|l|l|l|l|l|l|l|l|l|l|l|l|l|l|}
\hline 72.39 & 3.17 & 3.12 & 3.07 & 3.02 & 2.97 & 2.92 & 2.87 & 2.83 & 2.78 & 2.74 & 2.68 & 2.60 & 2.52 & 2.45 & 2.38 & 2.31 & 2.26 & 2.20 & 2.13 & 2.07 & 1.98 & 1.85 & 1.73 & 1.63 & 1.54 & 1.46 & 1.38 & 1.32 \\
\hline
\end{tabular} \begin{tabular}{|l|l|l|l|l|l|l|l|l|l|l|l|l|l|l|l|l|l|l|l|l|l|l|l|l|l|l|l|l|l|l|l|}
69.85 & 3.67 & 3.61 & 3.54 & 3.49 & 3.43 & 3.37 & 3.32 & 3.26 & 3.21 & 3.16 & 3.08 & 2.99 & 2.90 & 2.81 & 2.73 & 2.65 & 2.59 & 2.52 & 2.44 & 2.37 & 2.26 & 2.11 & 1.97 & 1.85 & 1.75 & 1.65 & 1.56 & 1.49 \\
\hline 67.31 & 4.13 & 4.06 & 3.9 & 3.92 & 3.85 & 3.7 & 3.73 & 3.67 & 3.61 & 3.55 & 3.46 & 3.36 & 3.25 & 3.15 & 3.06 & 2.97 & 2.90 & 2.82 & 2.72 & 2.65 & 2.52 & 2.36 & 2.19 & 2.05 & 1.94 & 1.83 & 1.73 & 1.65 &
\end{tabular} \begin{tabular}{|l|l|l|l|l|l|l|l|l|l|l|l|l|l|l|l|l|l|l|l|l|l|l|l|l|l|l|l|l|l|l|l|l|l|l|l|}
67.31 & 4.13 & 4.06 & 3.99 & 3.92 & 3.85 & 3.79 & 3.73 & 3.67 & 3.61 & 3.55 & 3.46 & 3.36 & 3.25 & 3.15 & 3.06 & 2.97 & 2.90 & 2.82 & 2.72 & 2.65 & 2.52 & 2.36 & 2.19 & 2.05 & 1.94 & 1.83 & 1.73 & 1.65 \\
\hline
\end{tabular} \begin{tabular}{|l|l|l|l|l|l|l|l|l|l|l|l|l|l|l|l|l|l|l|l|l|l|l|l|l|l|l|l|l|l|l|}
64.77 & 4.55 & 4.47 & 4.39 & 4.32 & 4.25 & 4.17 & 4.10 & 4.03 & 3.97 & 3.90 & 3.81 & 3.69 & 3.57 & 3.46 & 3.36 & 3.26 & 3.19 & 3.10 & 2.99 & 2.90 & 2.76 & 2.58 & 2.40 & 2.25 & 2.12 & 2.00 & 1.88 & 1.79 \\
\hline
\end{tabular} \begin{tabular}{|l|l|l|l|l|l|l|l|l|l|l|l|l|l|l|l|l|l|l|l|l|l|l|l|l|l|l|l|l|l|l|l|}
62.23 & 4.91 & 4.83 & 4.74 & 4.66 & 4.58 & 4.50 & 4.42 & 4.35 & 4.28 & 4.21 & 4.11 & 3.98 & 3.85 & 3.73 & 3.62 & 3.51 & 3.44 & 3.34 & 3.22 & 3.13 & 2.98 & 2.78 & 2.58 & 2.42 & 2.28 & 2.15 & 2.02 & 1.93 \\
\hline
\end{tabular} \begin{tabular}{|l|l|l|l|l|l|l|l|l|l|l|l|l|l|l|l|l|l|l|l|l|l|l|l|l|l|l|l|l|l|l|l|}
59.69 & 5.22 & 5.13 & 5.04 & 4.95 & 4.86 & 4.78 & 4.70 & 4.62 & 4.55 & 4.47 & 4.37 & 4.23 & 4.09 & 3.97 & 3.84 & 3.73 & 3.65 & 3.54 & 3.42 & 3.32 & 3.16 & 2.95 & 2.74 & 2.56 & 2.42 & 2.27 & 2.14 & 2.04 \\
\hline
\end{tabular}

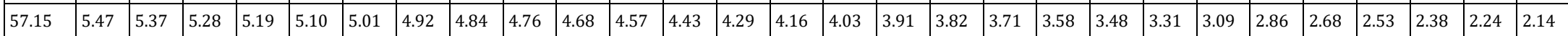
Axial

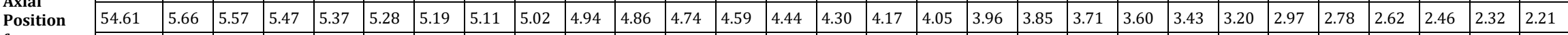

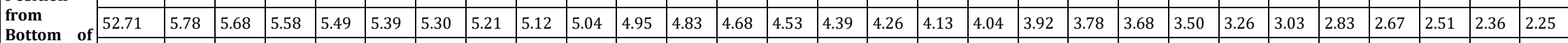
\begin{tabular}{|l|l|l|l|l|l|l|l|l|l|l|l|l|l|l|l|l|l|l|l|l|l|l|l|l|l|l|l|l|l|l|l|l|l|l|l|}
$\begin{array}{l}\text { Bottom of } \\
\text { beryllium }\end{array}$ & 51.44 & 5.83 & 5.73 & 5.63 & 5.53 & 5.44 & 5.35 & 5.26 & 5.17 & 5.08 & 5.00 & 4.88 & 4.72 & 4.57 & 4.43 & 4.30 & 4.17 & 4.08 & 3.96 & 3.82 & 3.71 & 3.53 & 3.29 & 3.05 & 2.86 & 2.70 & 2.54 & 2.39 & 2.27 \\
\hline (
\end{tabular}

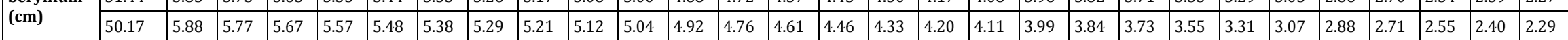
\begin{tabular}{|l|l|l|l|l|l|l|l|l|l|l|l|l|l|l|l|l|l|l|l|l|l|l|l|l|l|l|l|l|l|l}
\hline 48.90 & 5.90 & 5.80 & 5.70 & 5.60 & 5.50 & 5.41 & 5.31 & 5.22 & 5.14 & 5.05 & 4.93 & 4.77 & 4.62 & 4.48 & 4.34 & 4.21 & 4.12 & 4.00 & 3.86 & 3.75 & 3.56 & 3.32 & 3.08 & 2.89 & 2.73 & 2.56 & 2.41 & 2.30 \\
\hline
\end{tabular} \begin{tabular}{|l|l|l|l|l|l|l|l|l|l|l|l|l|l|l|l|l|l|l|l|l|l|l|l|l|l|l|l|l|l|}
47.63 & 5.91 & 5.81 & 5.70 & 5.60 & 5.51 & 5.41 & 5.33 & 5.24 & 5.15 & 5.07 & 4.94 & 4.78 & 4.63 & 4.49 & 4.35 & 4.22 & 4.13 & 4.01 & 3.86 & 3.75 & 3.57 & 3.33 & 3.09 & 2.89 & 2.73 & 2.56 & 2.42 & 2.30 \\
\hline
\end{tabular} \begin{tabular}{|l|l|l|l|l|l|l|l|l|l|l|l|l|l|l|l|l|l|l|l|l|l|l|l|l|l|l|l|l|l|l|l|}
\hline 46.36 & 5.90 & 5.80 & 5.70 & 5.60 & 5.50 & 5.41 & 5.32 & 5.23 & 5.14 & 5.06 & 4.94 & 4.78 & 4.63 & 4.49 & 4.35 & 4.22 & 4.12 & 4.01 & 3.87 & 3.75 & 3.57 & 3.33 & 3.09 & 2.89 & 2.73 & 2.57 & 2.41 & 2.29 \\
\hline
\end{tabular} \begin{tabular}{|l|l|l|l|l|l|l|l|l|l|l|l|l|l|l|l|l|l|l|l|l|l|l|l|l|l|l|l|l|l|l|}
45.09 & 5.88 & 5.77 & 5.67 & 5.58 & 5.48 & 5.39 & 5.30 & 5.21 & 5.13 & 5.04 & 4.92 & 4.77 & 4.61 & 4.47 & 4.34 & 4.20 & 4.11 & 3.99 & 3.85 & 3.74 & 3.56 & 3.32 & 3.08 & 2.88 & 2.72 & 2.55 & 2.40 & 2.28 \\
\hline
\end{tabular} \begin{tabular}{|l|l|l|l|l|l|l|l|l|l|l|l|l|l|l|l|l|l|l|l|l|l|l|l|l|l|l|l|l|l|l|l|}
43.82 & 5.84 & 5.74 & 5.65 & 5.55 & 5.45 & 5.36 & 5.27 & 5.18 & 5.10 & 5.01 & 4.89 & 4.74 & 4.59 & 4.45 & 4.31 & 4.18 & 4.09 & 3.97 & 3.83 & 3.72 & 3.53 & 3.30 & 3.06 & 2.86 & 2.70 & 2.54 & 2.39 & 2.27 \\
\hline
\end{tabular} \begin{tabular}{|l|l|l|l|l|l|l|l|l|l|l|l|l|l|l|l|l|l|l|l|l|l|l|l|l|l|l|l|l|l|}
42.55 & 5.79 & 5.69 & 5.59 & 5.49 & 5.40 & 5.31 & 5.23 & 5.14 & 5.05 & 4.97 & 4.85 & 4.69 & 4.55 & 4.41 & 4.27 & 4.15 & 4.05 & 3.94 & 3.80 & 3.69 & 3.51 & 3.27 & 3.04 & 2.84 & 2.68 & 2.52 & 2.37 & 2.25 \\
\hline 41.28 & 5.73 & 5.63 & 5.53 & 5.44 & 5.35 & 5.26 & 5.17 & 5.08 & 5.00 & 4.92 & 4.80 & 4.65 & 4.50 & 4.36 & 4.22 & 4.10 & 4.01 & 3.89 & 3.75 & 3.65 & 3.47 & 3.24 & 3.00 & 2.81 & 2.65 & 2.49 & 2.34 & 2.23 \\
\hline
\end{tabular} \begin{tabular}{|l|l|l|l|l|l|l|l|l|l|l|l|l|l|l|l|l|l|l|l|l|l|l|l|l|l|l|l|l|l|l|l|l}
41.28 & 5.73 & 5.63 & 5.53 & 5.44 & 5.35 & 5.26 & 5.17 & 5.08 & 5.00 & 4.92 & 4.80 & 4.65 & 4.50 & 4.36 & 4.22 & 4.10 & 4.01 & 3.89 & 3.75 & 3.65 & 3.47 & 3.24 & 3.00 & 2.81 & 2.65 & 2.49 & 2.34 & 2.23 \\
\hline
\end{tabular} \begin{tabular}{|l|l|l|l|l|l|l|l|l|l|l|l|l|l|l|l|l|l|l|l|l|l|l|l|l|l|l|l|l|l|l|l}
40.01 & 5.66 & 5.56 & 5.47 & 5.37 & 5.28 & 5.19 & 5.10 & 5.02 & 4.93 & 4.85 & 4.74 & 4.59 & 4.44 & 4.31 & 4.18 & 4.05 & 3.96 & 3.84 & 3.71 & 3.60 & 3.43 & 3.19 & 2.96 & 2.77 & 2.62 & 2.46 & 2.32 & 2.20 \\
\hline
\end{tabular}

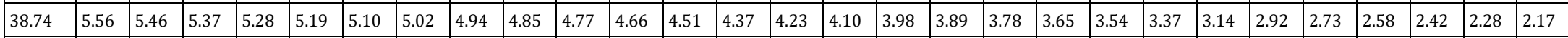
\begin{tabular}{|l|l|l|l|l|l|l|l|l|l|l|l|l|l|l|l|l|l|l|l|l|l|l|l|l|l|l|l|l|l|l|}
37.47 & 5.45 & 5.36 & 5.27 & 5.18 & 5.09 & 5.00 & 4.92 & 4.84 & 4.76 & 4.68 & 4.57 & 4.43 & 4.29 & 4.15 & 4.03 & 3.91 & 3.82 & 3.72 & 3.58 & 3.48 & 3.31 & 3.09 & 2.87 & 2.68 & 2.53 & 2.38 & 2.24 & 2.13 \\
\hline
\end{tabular} \begin{tabular}{|l|l|l|l|l|l|l|l|l|l|l|l|l|l|l|l|l|l|l|l|l|l|l|l|l|l|l|l|l|l|l|l|l|}
\hline 36.20 & 5.34 & 5.25 & 5.16 & 5.07 & 4.99 & 4.90 & 4.82 & 4.74 & 4.66 & 4.58 & 4.47 & 4.33 & 4.19 & 4.07 & 3.94 & 3.82 & 3.74 & 3.63 & 3.50 & 3.40 & 3.24 & 3.03 & 2.81 & 2.63 & 2.48 & 2.33 & 2.20 & 2.09 \\
\hline
\end{tabular} 
Table D.10. Neutron and photon heating in beryllium reflector with HEU core at $10 \mathrm{MW}$ over 8 years operation $\left(\mathrm{W} / \mathrm{cm}^{3}\right)$ -

\section{continued}

\begin{tabular}{|c|c|c|c|c|c|c|c|c|c|c|c|c|c|c|c|c|c|c|c|c|c|c|c|c|c|c|c|c|c|}
\hline \multicolumn{2}{|c|}{ Radial Segment } & 1 & 2 & 3 & 4 & 5 & 6 & 7 & 8 & 9 & 10 & 11 & 12 & 13 & 14 & 15 & 16 & 17 & 18 & 19 & 20 & 21 & 22 & 23 & 24 & 25 & 26 & 27 & 28 \\
\hline $\begin{array}{l}\text { Distance } \\
\text { beryllium (cm }\end{array}$ & through & 0.050 & 0.150 & 0.250 & 0.350 & 0.450 & 0.550 & 0.650 & 0.750 & 0.850 & 0.950 & 1.100 & 1.300 & 1.500 & 1.700 & 1.900 & 2.100 & 2.247 & 2.442 & 2.689 & 2.889 & 3.239 & 3.739 & 4.289 & 4.789 & 5.239 & 5.739 & 6.239 & 6.686 \\
\hline \multirow{18}{*}{$\begin{array}{l}\text { Axial } \\
\text { Position from } \\
\text { Bottom of } \\
\text { beryllium } \\
\text { (cm) }\end{array}$} & 34.93 & 5.21 & 5.12 & 5.03 & 4.95 & 4.86 & 4.78 & 4.70 & 4.62 & 4.55 & 4.47 & 4.37 & 4.23 & 4.10 & 3.97 & 3.85 & 3.73 & 3.65 & 3.55 & 3.42 & 3.33 & 3.16 & 2.95 & 2.74 & 2.56 & 2.42 & 2.28 & 2.14 & 2.04 \\
\hline & 33.66 & 5.08 & 4.99 & 4.90 & 4.82 & 4.74 & 4.66 & 4.58 & 4.50 & 4.43 & 4.36 & 4.25 & 4.12 & 3.99 & 3.86 & 3.75 & 3.64 & 3.56 & 3.45 & 3.33 & 3.24 & 3.08 & 2.87 & 2.67 & 2.50 & 2.36 & 2.21 & 2.08 & 1.98 \\
\hline & 32.39 & 4.92 & 4.84 & 4.75 & 4.67 & 4.59 & 4.52 & 4.44 & 4.37 & 4.30 & 4.23 & 4.13 & 4.00 & 3.87 & 3.75 & 3.64 & 3.53 & 3.45 & 3.35 & 3.23 & 3.14 & 2.99 & 2.79 & 2.59 & 2.42 & 2.29 & 2.15 & 2.03 & 1.93 \\
\hline & 31.12 & 4.76 & 4.68 & 4.60 & 4.52 & 4.45 & 4.37 & 4.30 & 4.23 & 4.16 & 4.09 & 3.99 & 3.86 & 3.74 & 3.63 & 3.52 & 3.41 & 3.34 & 3.24 & 3.13 & 3.04 & 2.89 & 2.70 & 2.51 & 2.35 & 2.22 & 2.08 & 1.96 & 1.86 \\
\hline & 29.85 & 4.58 & 4.50 & 4.43 & 4.35 & 4.28 & 4.21 & 4.14 & 4.07 & 4.01 & 3.94 & 3.85 & 3.73 & 3.61 & 3.50 & 3.39 & 3.29 & 3.22 & 3.13 & 3.02 & 2.93 & 2.79 & 2.61 & 2.42 & 2.27 & 2.14 & 2.01 & 1.89 & 1.80 \\
\hline & 28.58 & 4.40 & 4.33 & 4.25 & 4.18 & 4.11 & 4.04 & 3.97 & 3.91 & 3.84 & 3.78 & 3.69 & 3.58 & 3.46 & 3.36 & 3.26 & 3.16 & 3.09 & 3.00 & 2.90 & 2.82 & 2.68 & 2.50 & 2.33 & 2.18 & 2.06 & 1.94 & 1.83 & 1.74 \\
\hline & 27.31 & 4.20 & 4.13 & 4.06 & 3.99 & 3.92 & 3.86 & 3.79 & 3.73 & 3.67 & 3.61 & 3.53 & 3.42 & 3.31 & 3.21 & 3.11 & 3.02 & 2.96 & 2.87 & 2.77 & 2.69 & 2.57 & 2.40 & 2.23 & 2.09 & 1.97 & 1.86 & 1.75 & 1.67 \\
\hline & 26.04 & 4.00 & 3.93 & 3.86 & 3.80 & 3.73 & 3.67 & 3.61 & 3.55 & 3.50 & 3.44 & 3.36 & 3.25 & 3.15 & 3.05 & 2.96 & 2.88 & 2.82 & 2.74 & 2.64 & 2.57 & 2.45 & 2.28 & 2.13 & 2.00 & 1.89 & 1.78 & 1.68 & 1.60 \\
\hline & 24.77 & 3.79 & 3.72 & 3.66 & 3.60 & 3.54 & 3.48 & 3.42 & 3.36 & 3.31 & 3.25 & 3.18 & 3.08 & 2.99 & 2.90 & 2.81 & 2.73 & 2.67 & 2.60 & 2.50 & 2.44 & 2.32 & 2.17 & 2.02 & 1.89 & 1.79 & 1.69 & 1.60 & 1.52 \\
\hline & 23.50 & 3.56 & 3.50 & 3.44 & 3.38 & 3.33 & 3.27 & 3.21 & 3.16 & 3.11 & 3.06 & 2.99 & 2.90 & 2.81 & 2.73 & 2.64 & 2.57 & 2.51 & 2.44 & 2.36 & 2.30 & 2.19 & 2.05 & 1.91 & 1.79 & 1.70 & 1.60 & 1.51 & 1.45 \\
\hline & 22.23 & 3.33 & 3.27 & 3.22 & 3.16 & 3.11 & 3.06 & 3.01 & 2.96 & 2.91 & 2.87 & 2.80 & 2.71 & 2.63 & 2.55 & 2.48 & 2.40 & 2.35 & 2.29 & 2.21 & 2.15 & 2.05 & 1.92 & 1.80 & 1.69 & 1.60 & 1.51 & 1.43 & 1.37 \\
\hline & 20.96 & 3.08 & 3.03 & 2.98 & 2.93 & 2.88 & 2.83 & 2.79 & 2.74 & 2.69 & 2.65 & 2.59 & 2.51 & 2.44 & 2.37 & 2.30 & 2.24 & 2.19 & 2.13 & 2.06 & 2.00 & 1.91 & 1.79 & 1.68 & 1.58 & 1.50 & 1.42 & 1.34 & 1.29 \\
\hline & 19.69 & 2.82 & 2.77 & 2.72 & 2.68 & 2.64 & 2.59 & 2.55 & 2.51 & 2.47 & 2.43 & 2.38 & 2.31 & 2.24 & 2.18 & 2.11 & 2.06 & 2.01 & 1.96 & 1.90 & 1.85 & 1.77 & 1.66 & 1.55 & 1.47 & 1.39 & 1.32 & 1.25 & 1.20 \\
\hline & 18.42 & 2.53 & 2.49 & 2.45 & 2.41 & 2.38 & 2.34 & 2.30 & 2.27 & 2.24 & 2.20 & 2.16 & 2.10 & 2.04 & 1.98 & 1.93 & 1.87 & 1.84 & 1.79 & 1.74 & 1.69 & 1.62 & 1.53 & 1.43 & 1.35 & 1.29 & 1.22 & 1.16 & 1.12 \\
\hline & 16.51 & 2.11 & 2.08 & 2.05 & 2.02 & 1.99 & 1.97 & 1.94 & 1.91 & 1.89 & 1.86 & 1.83 & 1.78 & 1.74 & 1.69 & 1.65 & 1.61 & 1.58 & 1.55 & 1.50 & 1.47 & 1.41 & 1.33 & 1.26 & 1.19 & 1.14 & 1.09 & 1.04 & 1.00 \\
\hline & 12.70 & 1.46 & 1.44 & 1.43 & 1.41 & 1.39 & 1.38 & 1.36 & 1.35 & 1.33 & 1.32 & 1.30 & 1.27 & 1.24 & 1.22 & 1.19 & 1.17 & 1.15 & 1.13 & 1.10 & 1.08 & 1.05 & 1.00 & 0.95 & 0.91 & 0.87 & 0.84 & 0.80 & 0.78 \\
\hline & 7.62 & 0.91 & 0.90 & 0.89 & 0.89 & 0.88 & 0.87 & 0.86 & 0.85 & 0.84 & 0.84 & 0.83 & 0.81 & 0.80 & 0.79 & 0.77 & 0.76 & 0.75 & 0.74 & 0.72 & 0.71 & 0.70 & 0.67 & 0.64 & 0.62 & 0.60 & 0.58 & 0.57 & 0.55 \\
\hline & 2.54 & 0.58 & 0.57 & 0.57 & 0.57 & 0.56 & 0.56 & 0.55 & 0.55 & 0.54 & 0.54 & 0.53 & 0.53 & 0.52 & 0.51 & 0.51 & 0.50 & 0.50 & 0.49 & 0.48 & 0.48 & 0.47 & 0.45 & 0.44 & 0.43 & 0.42 & 0.41 & 0.40 & 0.39 \\
\hline
\end{tabular}


Table D.11. Neutron and photon heating in beryllium reflector with LEU core at $12 \mathrm{MW}$ over 8 years operation (W/cm $\left.{ }^{3}\right)$

\begin{tabular}{|c|c|c|c|c|c|c|c|c|c|c|c|c|c|c|c|c|c|c|c|c|c|c|c|c|c|c|c|c|c|}
\hline \multicolumn{2}{|l|}{ Radial Segment } & 1 & 2 & 3 & 4 & 5 & 6 & 7 & 8 & 9 & 10 & 11 & 12 & 13 & 14 & 15 & 16 & 17 & 18 & 19 & 20 & 21 & 22 & 23 & 24 & 25 & 26 & 27 & 28 \\
\hline $\begin{array}{l}\text { Distance } \\
\text { beryllium (cm) }\end{array}$ & $\begin{array}{l}\text { through } \\
\text { i) }\end{array}$ & 0.050 & 0.150 & 0.250 & 0.350 & 0.450 & 0.550 & 0.650 & 0.750 & 0.850 & 0.950 & 1.100 & 1.300 & 1.500 & 1.700 & 1.900 & 2.100 & 2.247 & 2.442 & 2.689 & 2.889 & 3.239 & 3.739 & 4.289 & 4.789 & 5.239 & 5.739 & 6.239 & 6.686 \\
\hline & 91.44 & 0.47 & 0.46 & 0.46 & 0.46 & 0.45 & 0.45 & 0.44 & 0.44 & \begin{tabular}{|l|l|}
0.44 \\
\end{tabular} & 0.43 & 0.43 & 0.42 & 0.42 & 0.41 & 0.40 & 0.39 & \begin{tabular}{|l|l|}
0.39 \\
\end{tabular} & 0.38 & 0.38 & 0.37 & 0.36 & 0.35 & 0.34 & 0.34 & 0.33 & 0.33 & 0.32 & 0.31 \\
\hline & 86.36 & 0.76 & 0.75 & 0.74 & 0.73 & 0.73 & 0.72 & 0.71 & 0.70 & 0.70 & 0.69 & 0.68 & 0.67 & 0.66 & 0.64 & 0.62 & 0.61 & 0.60 & 0.59 & 0.58 & $\mid 0.57$ & 0.55 & 0.53 & 0.51 & 0.50 & 0.49 & 0.48 & 0.46 & 0.45 \\
\hline & 81.28 & 1.24 & 1.22 & 1.21 & 1.19 & 1.17 & 1.16 & 1.15 & 1.13 & 1.12 & 1.11 & 1.09 & 1.06 & 1.04 & 1.01 & 0.98 & 0.96 & 0.94 & 0.92 & 0.89 & 0.87 & 0.84 & 0.81 & 0.77 & 0.74 & 0.72 & 0.70 & 0.67 & 0.65 \\
\hline & 77.47 & 1.81 & 1.78 & 1.76 & 1.73 & 1.70 & 1.68 & 1.65 & 1.63 & 1.61 & 1.58 & 1.55 & 1.51 & 1.47 & 1.43 & 1.40 & 1.36 & 1.34 & 1.30 & 1.27 & 1.24 & 1.19 & 1.12 & 1.06 & 1.00 & 0.96 & 0.91 & 0.87 & 0.84 \\
\hline & 74.93 & 2.27 & 2.24 & 2.20 & 2.16 & 2.13 & 2.09 & 2.06 & 2.03 & 2.00 & 1.97 & 1.92 & 1.87 & 1.81 & 1.76 & 1.71 & 1.67 & 1.64 & 1.60 & 1.54 & 1.50 & 1.44 & 1.36 & 1.27 & 1.20 & 1.14 & 1.08 & 1.03 & 0.99 \\
\hline & 72.39 & 2.73 & 2.68 & 2.64 & 2.59 & 2.55 & 2.51 & 2.47 & 2.43 & 2.39 & 2.36 & 2.30 & 2.23 & 2.17 & 2.10 & 2.04 & 1.99 & 1.95 & 1.89 & 1.83 & 1.78 & 1.70 & 1.59 & 1.49 & 1.40 & 1.33 & 1.26 & 1.19 & 1.14 \\
\hline & 69.85 & 3.18 & 3.13 & 3.07 & 3.02 & 2.97 & 2.92 & 2.87 & 2.83 & 2.78 & 2.74 & 2.67 & 2.59 & 2.51 & 2.43 & 2.36 & 2.29 & 2.25 & 2.18 & 2.11 & 2.05 & 1.95 & 1.83 & 1.70 & 1.60 & 1.51 & 1.43 & 1.35 & 1.30 \\
\hline & 67.31 & 3.60 & 3.53 & 3.47 & 3.41 & 3.36 & 3.30 & 3.24 & 3.19 & 3.14 & 3.09 & 3.01 & 2.92 & 2.82 & 2.74 & 2.66 & 2.58 & 2.52 & 2.45 & 2.37 & 2.30 & 2.19 & 2.05 & 1.90 & 1.79 & 1.69 & 1.59 & 1.51 & 1.44 \\
\hline & 64.77 & 3.96 & 3.89 & 3.82 & 3.76 & 3.69 & 3.63 & 3.57 & 3.51 & 3.45 & 3.39 & 3.31 & 3.21 & 3.11 & 3.01 & 2.92 & 2.83 & 2.77 & 2.69 & 2.60 & 2.52 & 2.40 & 2.24 & 2.09 & 1.96 & 1.85 & 1.74 & 1.65 & 1.57 \\
\hline & 62.23 & 4.28 & 4.20 & 4.13 & 4.06 & 3.99 & 3.92 & 3.86 & 3.79 & 3.73 & 3.67 & 3.58 & 3.47 & 3.35 & 3.25 & 3.15 & 3.06 & 2.99 & 2.91 & 2.80 & 2.72 & 2.59 & 2.42 & 2.25 & 2.11 & 1.99 & 1.87 & 1.77 & 1.69 \\
\hline & 59.69 & 4.54 & 4.46 & 4.39 & 4.31 & 4.23 & 4.16 & 4.10 & 4.03 & 3.96 & 3.90 & 3.80 & 3.68 & 3.57 & 3.45 & 3.35 & 3.25 & 3.18 & 3.09 & 2.98 & 2.89 & 2.75 & 2.57 & 2.38 & 2.23 & 2.11 & 1.99 & 1.88 & 1.79 \\
\hline & 57.15 & 4.76 & 4.68 & 4.60 & 4.52 & 4.44 & 4.37 & 4.29 & 4.22 & 4.15 & 4.08 & 3.98 & 3.86 & 3.73 & 3.62 & 3.51 & 3.40 & 3.33 & 3.23 & 3.12 & 3.03 & 2.88 & 2.69 & 2.50 & 2.34 & 2.21 & 2.08 & 1.97 & 1.87 \\
\hline - & 54.61 & 4.93 & 4.85 & 4.76 & 4.68 & 4.60 & 4.52 & 4.44 & 4.37 & 4.30 & 4.22 & 4.12 & 3.99 & 3.86 & 3.75 & 3.63 & 3.52 & 3.45 & 3.35 & 3.23 & 3.13 & 2.98 & 2.78 & 2.59 & 2.42 & 2.29 & 2.15 & 2.03 & 1.94 \\
\hline Bottom of & 52.71 & 5.02 & 4.93 & 4.85 & 4.76 & 4.68 & 4.60 & 4.53 & 4.45 & 4.38 & 4.31 & 4.20 & 4.07 & 3.94 & 3.82 & 3.70 & 3.59 & 3.51 & 3.41 & 3.29 & 3.20 & 3.04 & 2.84 & 2.64 & 2.47 & 2.33 & 2.19 & 2.07 & 1.97 \\
\hline (cm & 51.44 & 5.07 & 4.98 & 4.89 & 4.81 & 4.73 & 4.65 & 4.57 & 4.49 & 4.42 & 4.35 & 4.24 & 4.10 & 3.98 & 3.86 & 3.74 & 3.63 & 3.54 & 3.44 & 3.32 & 3.23 & 3.07 & 2.86 & 2.66 & 2.49 & 2.35 & 2.22 & 2.09 & 1.99 \\
\hline & 50.17 & 5.10 & 5.01 & 4.93 & 4.84 & 4.76 & 4.68 & 4.60 & 4.52 & 4.45 & 4.38 & 4.27 & 4.13 & 4.00 & 3.88 & 3.76 & 3.65 & 3.57 & 3.47 & 3.34 & 3.25 & 3.09 & 2.88 & 2.68 & 2.51 & 2.37 & 2.23 & 2.10 & 2.00 \\
\hline & 48.90 & 5.11 & 5.03 & 4.94 & 4.86 & 4.78 & 4.69 & 4.62 & 4.54 & 4. & 4.39 & 4.2 & 4. & 4.02 & 3.90 & 3.78 & 3.66 & 3.58 & 3.48 & 3.35 & 3.26 & 3.10 & 2.89 & 2.69 & 2.52 & 2.38 & 2.24 & 2.11 & 2.01 \\
\hline & 47.63 & 5.11 & 5.03 & 4.94 & 4.86 & 4.78 & 4.70 & 4.62 & 4.54 & 4.47 & 4.40 & 4.29 & 4.15 & 4.02 & 3.90 & 3.78 & 3.66 & 3.59 & 3.48 & 3.36 & 3.26 & 3.10 & 2.90 & 2.69 & 2.52 & 2.38 & 2.24 & 2.11 & 2.01 \\
\hline & 46.36 & 5.11 & 5.02 & 4.94 & 4.85 & 4.77 & 4.69 & 4.61 & 4.54 & 4.47 & 4.39 & 4.28 & 4.14 & 4.02 & 3.89 & 3.77 & 3.66 & 3.58 & 3.48 & 3.35 & 3.26 & 3.10 & 2.89 & 2.68 & 2.52 & 2.38 & 2.23 & 2.11 & 2.01 \\
\hline & 45.09 & 5.10 & 5.01 & 4.92 & 4.83 & 4.75 & 4.67 & 4.59 & 4.52 & 4.44 & 4.37 & 4.26 & 4.13 & 4.00 & 3.88 & 3.76 & 3.64 & 3.57 & 3.46 & 3.34 & 3.24 & 3.09 & 2.88 & 2.67 & 2.51 & 2.37 & 2.22 & 2.10 & 2.00 \\
\hline & 43.82 & 5.05 & 4.97 & 4.89 & 4.80 & 4.72 & 4.64 & 4.56 & 4.49 & 4.41 & 4.34 & 4.24 & 4.10 & 3.97 & 3.85 & 3.74 & 3.62 & 3.54 & 3.44 & 3.32 & 3.23 & 3.07 & 2.86 & 2.66 & 2.49 & 2.35 & 2.21 & 2.09 & 1.99 \\
\hline & 42.55 & 5.01 & 4.93 & 4.84 & 4.76 & 4.68 & 4.60 & 4.52 & 4.45 & 4.38 & 4.30 & 4.20 & 4.07 & 3.94 & 3.82 & 3.70 & 3.59 & 3.51 & 3.41 & 3.29 & 3.20 & 3.04 & 2.84 & 2.64 & 2.47 & 2.33 & 2.19 & 2.07 & 1.97 \\
\hline & 41.28 & 4.95 & 4.87 & 4.79 & 4.71 & 4.63 & 4.55 & 4.47 & 4.40 & 4.33 & 4.25 & 4.15 & 4.02 & 3.89 & 3.77 & 3.66 & 3.55 & 3.47 & 3.37 & 3.25 & 3.16 & 3.01 & 2.81 & 2.61 & 2.44 & 2.31 & 2.17 & 2.05 & 1.95 \\
\hline & 40.01 & 4.89 & 4.81 & 4.72 & 4.64 & 4.56 & 4.49 & 4.41 & 4.34 & 4.27 & 4.20 & 4.10 & 3.97 & 3.84 & 3.72 & 3.61 & 3.50 & 3.42 & 3.33 & 3.21 & 3.11 & 2.97 & 2.77 & 2.57 & 2.41 & 2.28 & 2.14 & 2.02 & 1.92 \\
\hline & 38.74 & 4.81 & 4.73 & 4.65 & 4.57 & 4.49 & 4.42 & 4.34 & 4.27 & 4.20 & 4.13 & 4.03 & 90 & 3.78 & 3.66 & 3.55 & 45 & 3.37 & 3.27 & 3.16 & 3.07 & 2.92 & 2.72 & 2.53 & 2.37 & 2.24 & 2.11 & 1.99 & 1.90 \\
\hline & 37.47 & 4.71 & 4.63 & 4.56 & 4.48 & 4.40 & 4.32 & 4.26 & 4.18 & 4.12 & 4.05 & 3.95 & 32 & 3.70 & 3.59 & 3.48 & 38 & 31 & 3.21 & 10 & 3.01 & 2.87 & 2.67 & 2.48 & 2.33 & 2.20 & 2.07 & 1.95 & 1.86 \\
\hline & 36.20 & 4.61 & 4.53 & 4.45 & 4.38 & 4.30 & 4.23 & 4.15 & 4.09 & 4.02 & 3.95 & 3.86 & 3.74 & 3.62 & 3.51 & 3.40 & 3.30 & 3.23 & 3.14 & 3.03 & 2.94 & 2.80 & 2.61 & 2.43 & 2.27 & 2.15 & 2.02 & 1.91 & 1.82 \\
\hline
\end{tabular}


Table D.12. Neutron and photon heating in beryllium reflector with LEU core at $12 \mathrm{MW}$ over 8 years operation (W/cm ${ }^{3}$ -

\section{continued}

\begin{tabular}{|c|c|c|c|c|c|c|c|c|c|c|c|c|c|c|c|c|c|c|c|c|c|c|c|c|c|c|c|c|c|}
\hline \multicolumn{2}{|c|}{ Radial Segment } & 1 & 2 & 3 & 4 & 5 & 6 & 7 & 8 & 9 & 10 & 11 & 12 & 13 & 14 & 15 & 16 & 17 & 18 & 19 & 20 & 21 & 22 & 23 & 24 & 25 & 26 & 27 & 28 \\
\hline $\begin{array}{l}\text { Distance } \\
\text { beryllium (cm }\end{array}$ & through & 0.050 & 0.150 & 0.250 & 0.350 & 0.450 & 0.550 & 0.650 & 0.750 & 0.850 & 0.950 & 1.100 & 1.300 & 1.500 & 1.700 & 1.900 & 2.100 & 2.247 & 2.442 & 2.689 & 2.889 & 3.239 & 3.739 & 4.289 & 4.789 & 5.239 & 5.739 & 6.239 & 6.686 \\
\hline \multirow{18}{*}{$\begin{array}{l}\text { Axial } \\
\text { Position from } \\
\text { Bottom of } \\
\text { beryllium } \\
\text { (cm) }\end{array}$} & 34.93 & 4.50 & 4.42 & 4.34 & 4.27 & 4.20 & 4.13 & 4.06 & 3.99 & 3.93 & 3.86 & 3.77 & 3.65 & 3.54 & 3.42 & 3.32 & 3.22 & 3.15 & 3.06 & 2.95 & 2.87 & 2.73 & 2.55 & 2.37 & 2.22 & 2.10 & 1.97 & 1.86 & 1.77 \\
\hline & 33.66 & 4.37 & 4.30 & 4.23 & 4.15 & 4.08 & 4.01 & 3.95 & 3.88 & 3.82 & 3.76 & 3.67 & 3.55 & 3.44 & 3.33 & 3.23 & 3.14 & 3.07 & 2.98 & 2.88 & 2.79 & 2.66 & 2.48 & 2.31 & 2.16 & 2.04 & 1.92 & 1.81 & 1.73 \\
\hline & 32.39 & 4.24 & 4.16 & 4.10 & 4.02 & 3.96 & 3.89 & 3.83 & 3.76 & 3.70 & 3.64 & 3.56 & 3.44 & 3.34 & 3.23 & 3.14 & 3.04 & 2.98 & 2.89 & 2.79 & 2.71 & 2.58 & 2.41 & 2.24 & 2.10 & 1.98 & 1.86 & 1.76 & 1.67 \\
\hline & 31.12 & 4.09 & 4.02 & 3.95 & 3.88 & 3.82 & 3.76 & 3.69 & 3.63 & 3.57 & 3.51 & 3.43 & 3.32 & 3.22 & 3.12 & 3.03 & 2.94 & 2.88 & 2.79 & 2.69 & 2.62 & 2.49 & 2.33 & 2.16 & 2.03 & 1.92 & 1.80 & 1.70 & 1.62 \\
\hline & 29.85 & 3.94 & 3.87 & 3.81 & 3.74 & 3.68 & 3.62 & 3.56 & 3.50 & 3.44 & 3.39 & 3.30 & 3.20 & 3.10 & 3.01 & 2.92 & 2.83 & 2.77 & 2.69 & 2.60 & 2.52 & 2.40 & 2.24 & 2.08 & 1.95 & 1.85 & 1.74 & 1.64 & 1.57 \\
\hline & 28.58 & 3.78 & 3.71 & 3.65 & 3.59 & 3.53 & 3.47 & 3.41 & 3.36 & 3.30 & 3.25 & 3.17 & 3.07 & 2.98 & 2.88 & 2.80 & 2.71 & 2.66 & 2.58 & 2.49 & 2.42 & 2.30 & 2.15 & 2.00 & 1.88 & 1.77 & 1.67 & 1.58 & 1.51 \\
\hline & 27.31 & 3.61 & 3.54 & 3.48 & 3.42 & 3.36 & 3.31 & 3.25 & 3.20 & 3.15 & 3.09 & 3.02 & 2.93 & 2.84 & 2.75 & 2.67 & 2.59 & 2.53 & 2.46 & 2.38 & 2.31 & 2.20 & 2.06 & 1.92 & 1.80 & 1.70 & 1.60 & 1.51 & 1.44 \\
\hline & 26.04 & 3.42 & 3.36 & 3.31 & 3.25 & 3.20 & 3.15 & 3.10 & 3.04 & 2.99 & 2.95 & 2.88 & 2.79 & 2.70 & 2.62 & 2.54 & 2.46 & 2.41 & 2.35 & 2.26 & 2.20 & 2.10 & 1.96 & 1.82 & 1.71 & 1.62 & 1.53 & 1.45 & 1.38 \\
\hline & 24.77 & 3.23 & 3.18 & 3.13 & 3.07 & 3.02 & 2.97 & 2.92 & 2.87 & 2.83 & 2.78 & 2.71 & 2.63 & 2.55 & 2.47 & 2.40 & 2.33 & 2.28 & 2.22 & 2.14 & 2.08 & 1.99 & 1.86 & 1.73 & 1.62 & 1.54 & 1.45 & 1.38 & 1.32 \\
\hline & 23.50 & 3.04 & 2.99 & 2.94 & 2.89 & 2.84 & 2.79 & 2.74 & 2.70 & 2.66 & 2.61 & 2.55 & 2.47 & 2.40 & 2.33 & 2.26 & 2.19 & 2.14 & 2.09 & 2.01 & 1.96 & 1.87 & 1.75 & 1.63 & 1.53 & 1.45 & 1.37 & 1.30 & 1.25 \\
\hline & 22.23 & 2.83 & 2.78 & 2.74 & 2.69 & 2.65 & 2.60 & 2.56 & 2.52 & 2.48 & 2.44 & 2.38 & 2.31 & 2.24 & 2.17 & 2.11 & 2.05 & 2.00 & 1.95 & 1.88 & 1.83 & 1.75 & 1.64 & 1.53 & 1.44 & 1.37 & 1.29 & 1.23 & 1.18 \\
\hline & 20.96 & 2.61 & 2.57 & 2.53 & 2.48 & 2.44 & 2.40 & 2.36 & 2.33 & 2.29 & 2.25 & 2.20 & 2.13 & 2.07 & 2.01 & 1.95 & 1.90 & 1.86 & 1.81 & 1.74 & 1.70 & 1.62 & 1.52 & 1.42 & 1.34 & 1.28 & 1.21 & 1.15 & 1.11 \\
\hline & 19.69 & 2.38 & 2.34 & 2.30 & 2.26 & 2.23 & 2.19 & 2.16 & 2.12 & 2.09 & 2.06 & 2.01 & 1.95 & 1.89 & 1.84 & 1.79 & 1.74 & 1.71 & 1.66 & 1.61 & 1.57 & 1.50 & 1.41 & 1.32 & 1.25 & 1.19 & 1.13 & 1.07 & 1.03 \\
\hline & 18.42 & 2.13 & 2.10 & 2.06 & 2.03 & 2.00 & 1.96 & 1.93 & 1.91 & 1.87 & 1.85 & 1.81 & 1.76 & 1.71 & 1.67 & 1.62 & 1.58 & 1.55 & 1.51 & 1.47 & 1.43 & 1.37 & 1.29 & 1.21 & 1.15 & 1.10 & 1.04 & 0.99 & 0.96 \\
\hline & 16.51 & 1.78 & 1.75 & 1.73 & 1.70 & 1.68 & 1.66 & 1.63 & 1.61 & 1.59 & 1.57 & 1.54 & 1.50 & 1.46 & 1.43 & 1.39 & 1.36 & 1.33 & 1.31 & 1.27 & 1.24 & 1.19 & 1.13 & 1.07 & 1.01 & 0.97 & 0.92 & 0.88 & 0.85 \\
\hline & 12.70 & 1.25 & 1.24 & 1.22 & 1.21 & 1.19 & 1.18 & 1.16 & 1.15 & 1.14 & 1.13 & 1.11 & 1.08 & 1.06 & 1.04 & 1.02 & 1.00 & 0.98 & 0.96 & 0.94 & 0.92 & 0.89 & 0.85 & 0.81 & 0.78 & 0.75 & 0.72 & 0.69 & 0.67 \\
\hline & 7.62 & 0.80 & 0.79 & 0.78 & 0.77 & 0.76 & 0.76 & 0.75 & 0.74 & 0.73 & 0.73 & 0.72 & 0.71 & 0.69 & 0.68 & 0.67 & 0.66 & 0.65 & 0.64 & 0.63 & 0.62 & 0.60 & 0.58 & 0.56 & 0.54 & 0.53 & 0.51 & 0.49 & 0.48 \\
\hline & 2.54 & 0.52 & 0.51 & 0.51 & 0.50 & 0.50 & 0.49 & 0.49 & 0.49 & 0.48 & 0.48 & 0.47 & 0.47 & 0.46 & 0.46 & 0.45 & 0.44 & 0.44 & 0.43 & 0.43 & 0.42 & 0.41 & 0.40 & 0.39 & 0.38 & 0.37 & 0.36 & 0.36 & 0.35 \\
\hline
\end{tabular}




\section{APPENDIX E: Conversion of Data from Various Reactor Sites}

\section{E.1 SM Reactor}

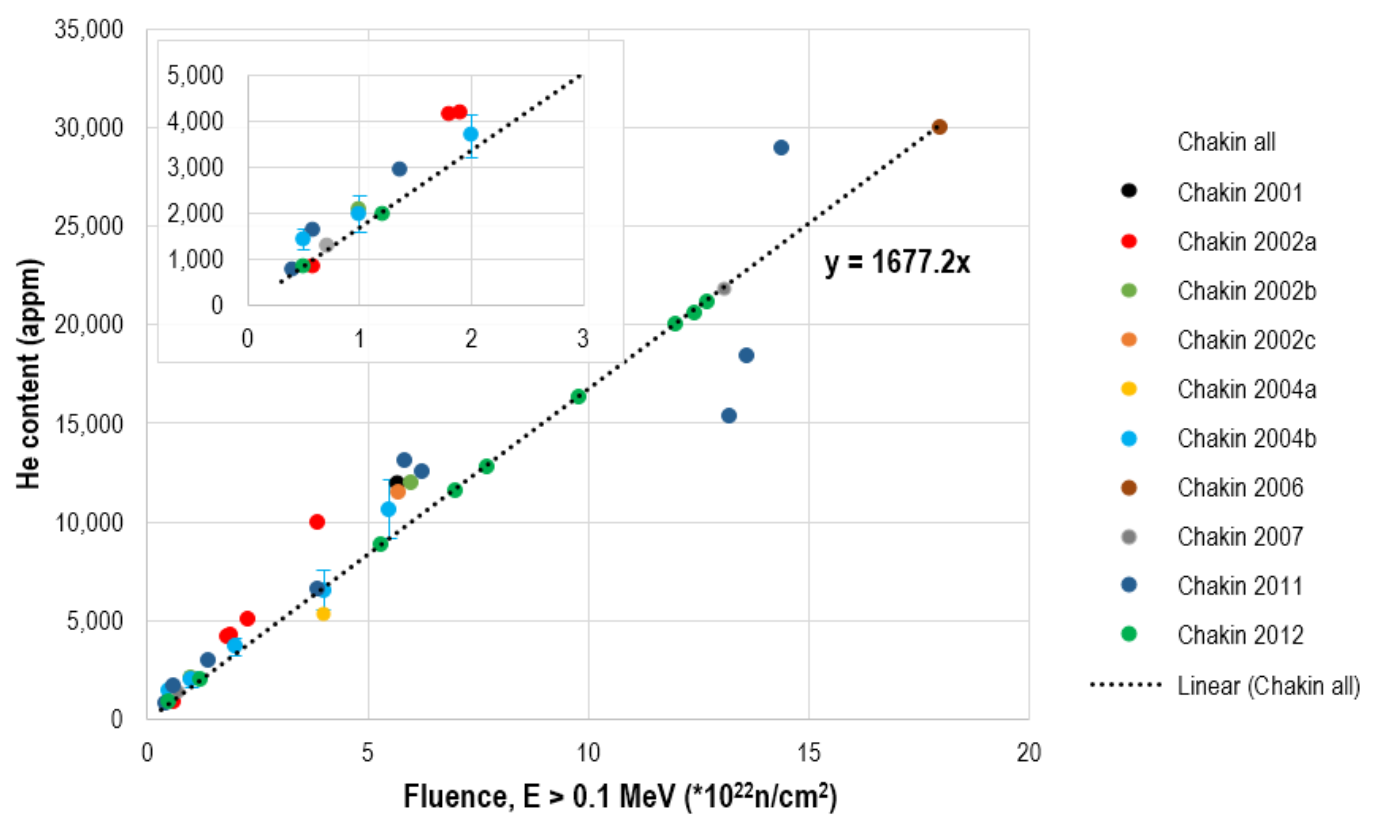

Figure E.1. Relationship between fluence $(\mathrm{E}>0.1 \mathrm{MeV}$ ) and He-4 content for SM beryllium reflector

There is only one data point available that allows to relate fluence $\mathrm{E}>0.1 \mathrm{MeV}$ to corresponding $\mathrm{E}>1.0$ $\mathrm{MeV}$ in SM reactor. There is about 2.03 times more neutrons with $\mathrm{E}>0.1 \mathrm{MeV}$ than the neutrons with $\mathrm{E}>1 \mathrm{MeV}[56]$. 


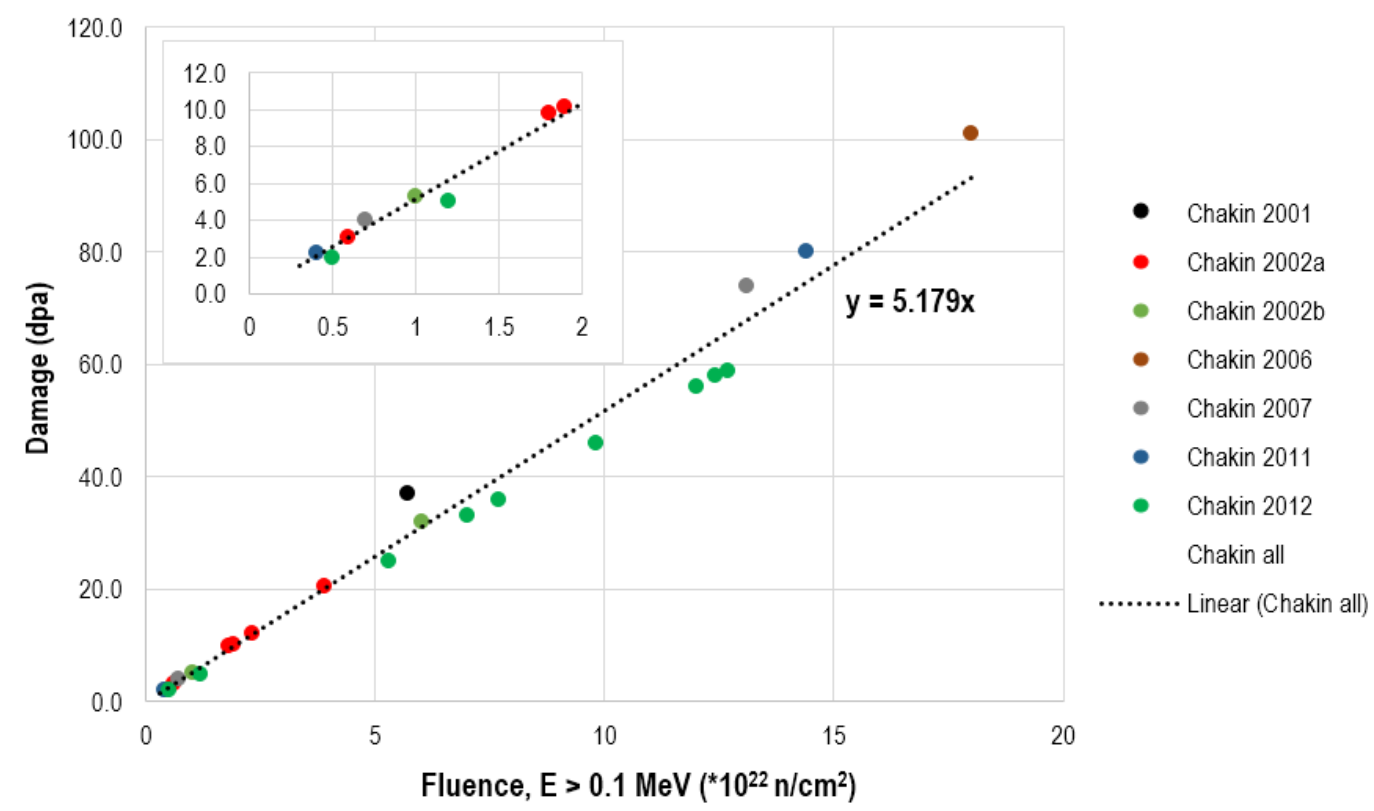

Figure E.2. Relationship between fluence $(\mathrm{E}>0.1 \mathrm{MeV})$ and neutron damage for SM beryllium reflector

\section{E.2 BR2 Reactor}

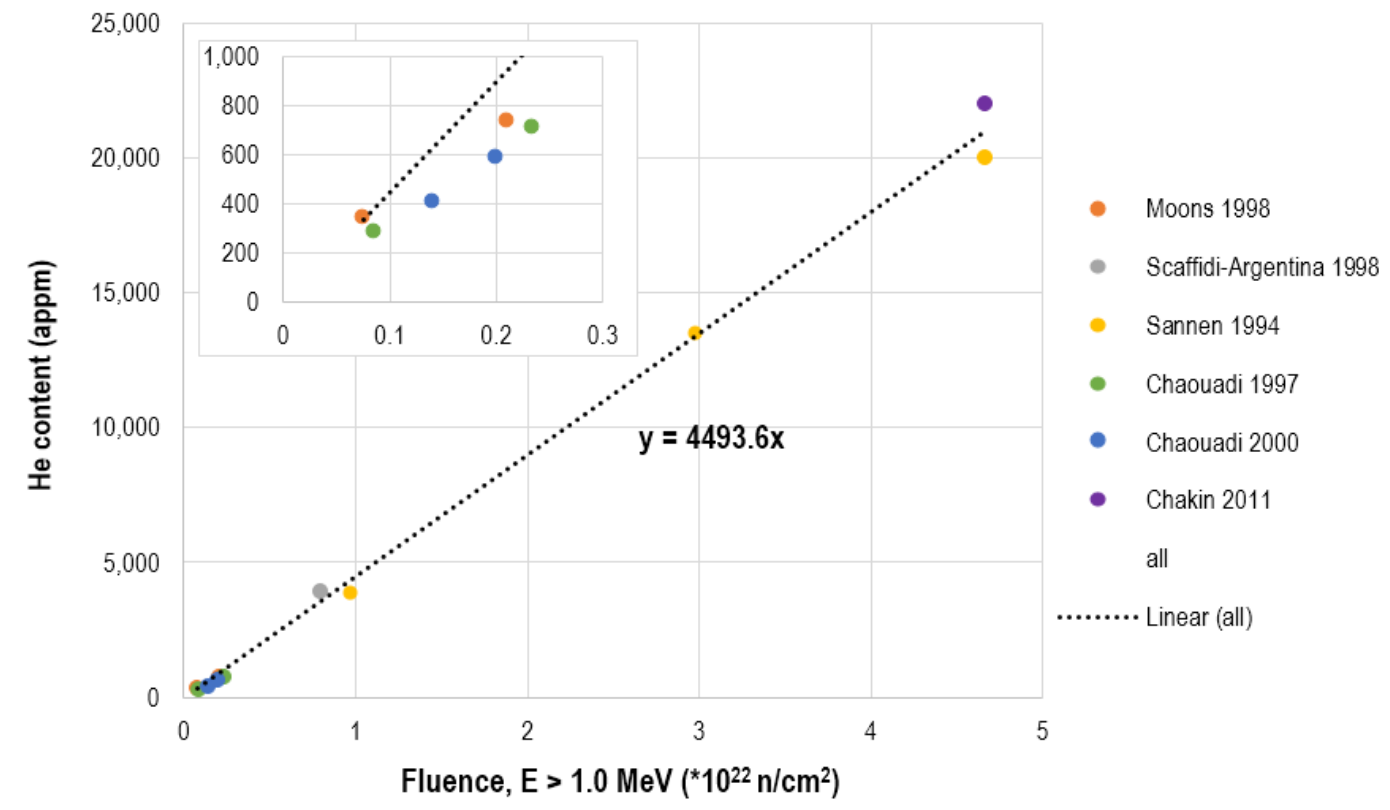

Figure E.3. Relationship between fluence $(\mathrm{E}>1.0 \mathrm{MeV})$ and helium concentration for BR2 beryllium reflector 


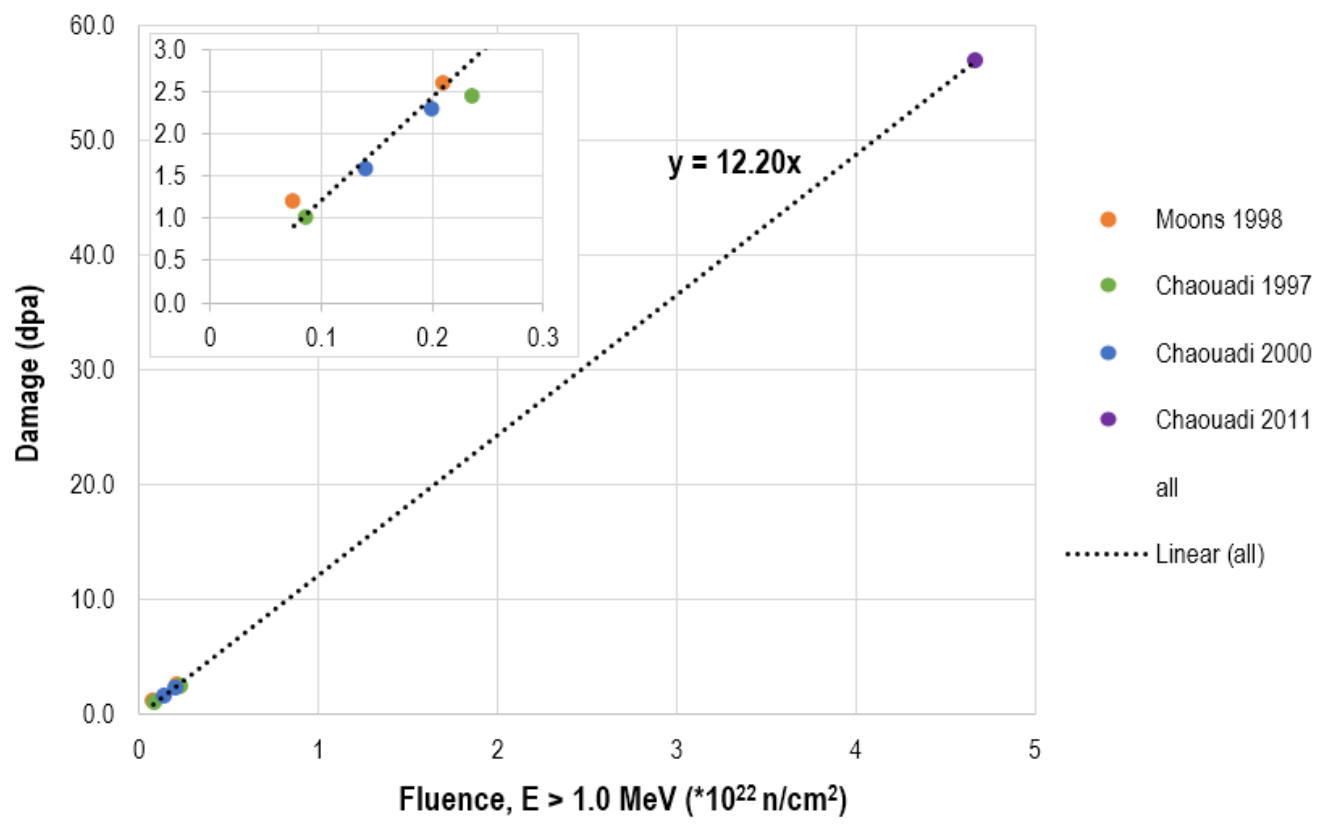

Figure E.4. Relationship between fluence $(\mathrm{E}>1.0 \mathrm{MeV})$ and neutron damage for BR2 beryllium reflector

\section{E.3 HFIR Reactor}

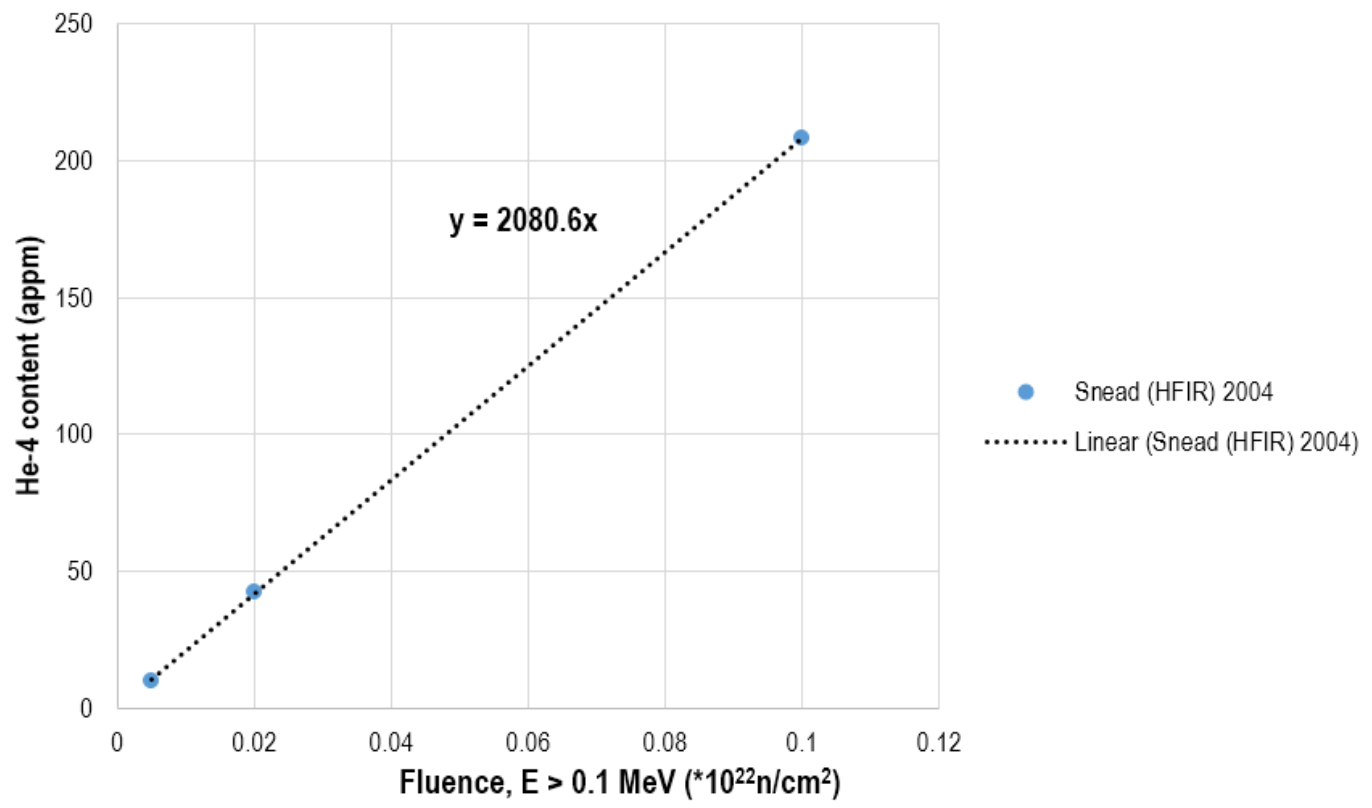

Figure E.5. Relationship between fluence ( $\mathrm{E}>0.1 \mathrm{MeV}$ ) and helium concertation for HFIR beryllium reflector 


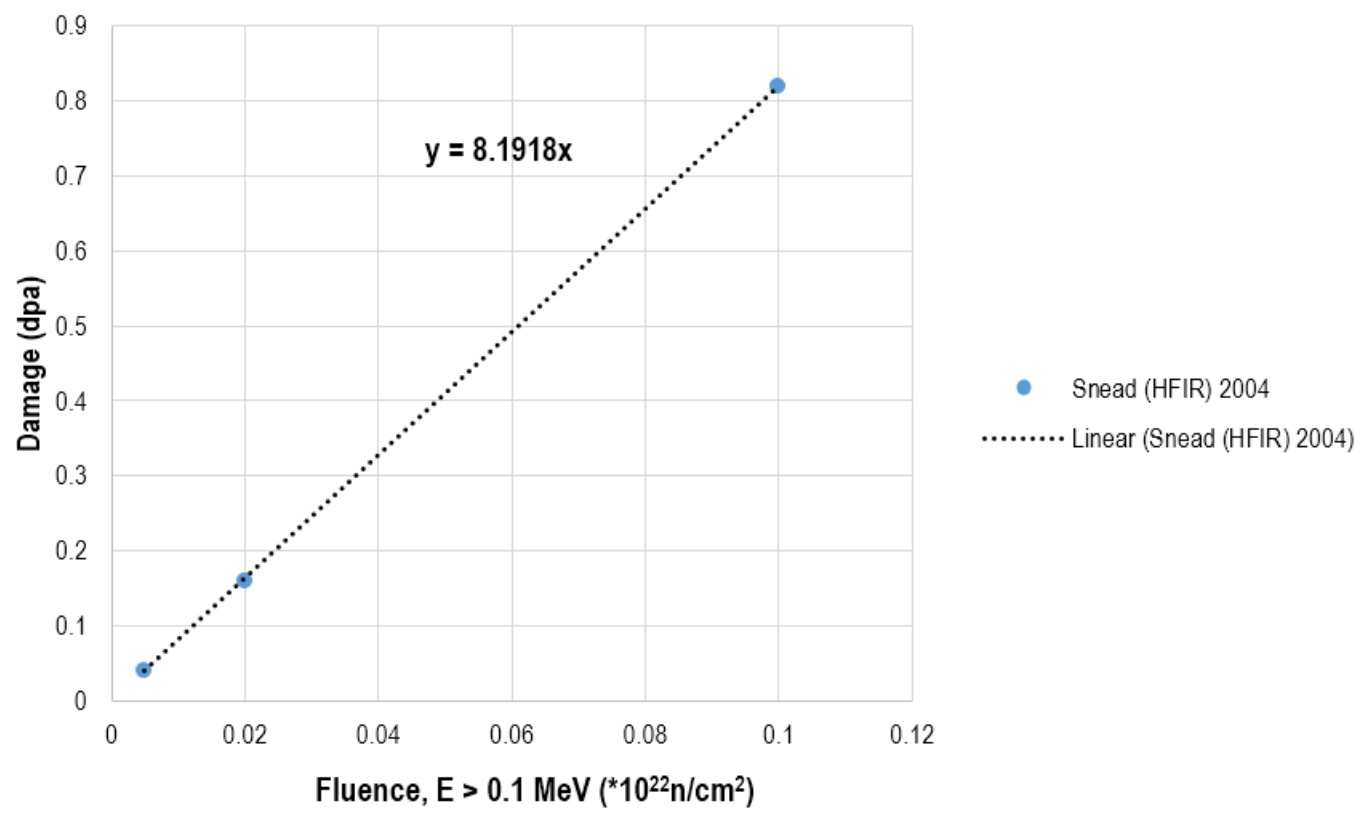

Figure E.6. Relationship between fluence $(\mathrm{E}>0.1 \mathrm{MeV})$ and neutron damage for HFIR beryllium reflector

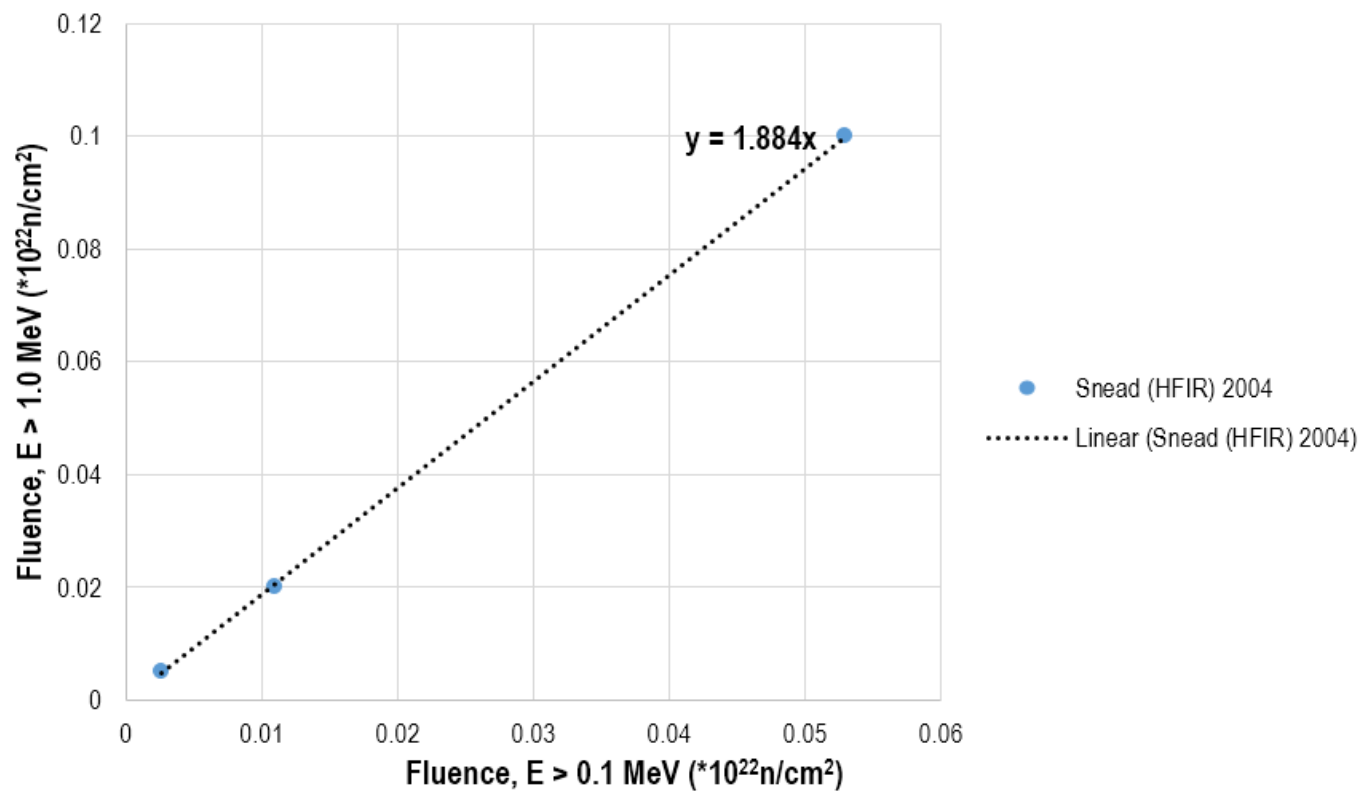

Figure E.7. Relationship between fluence $(\mathrm{E}>0.1 \mathrm{MeV})$ and fluence $(\mathrm{E}>1.0 \mathrm{MeV})$ for HFIR beryllium reflector 




\section{Argonne $\mathbf{A}$}

Nuclear Science \& Engineering Division

Argonne National Laboratory

9700 South Cass Avenue, Bldg. 208

Argonne, IL 60439

www.anl.gov

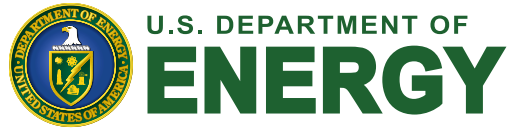

Argonne National Laboratory is a U.S. Department of Energy laboratory managed by UChicago Argonne, LLC 